\title{
37th MEETING OF THE CANADIAN CONGRESS OF NEUROLOGICAL SCIENCES
}

\author{
JUNE 18-22, 2002 \\ VANCOUVER, BRITISH COLUMBIA
}

\begin{abstract}
s
Society Prize Presentations

Canadian Neurological Society - Frances McNaughton Memorial Prize

Canadian Association of Child Neurology - President's Prize

Canadian Neurosurgical Society - K.G. McKenzie Prize in Clinical Neuroscience Research

Canadian Neurosurgical Society - K.G. McKenzie Prize in Basic Neuroscience Research
\end{abstract}

\section{Platform Presentations}

Thursday June 20, 2002
Friday June 21, 2002
A. Tumour ...
A-01 to $\mathrm{A}-07$
I. Child Neurology
I-01 to $\mathrm{I}-08$
B. Spinal and Peripheral Nereve
B-01 to B-07
J. General Neurosurgery
$\mathrm{J}-01$ to $\mathrm{J}-08$
C. Multiple Sclerosis C-01 to $\mathrm{C}-07$
K. Stroke $\mathrm{K}-01$ to $\mathrm{K}-08$
D. Epilepsy D-01 to D-07
L. Vascular Neurosurgery L-01 to $\mathrm{L}-08$
E. Basic Neuroscience/Neurology.... E-01 to E-06
F. Neurophysiology $\mathrm{F}-01$ to $\mathrm{F}-06$
G. Pediatric Neurosurgery G-01 to G-06
H. Movement Disorders $\mathrm{H}-01$ to $\mathrm{H}-06$

\section{Poster Presentations}

Thursday June 20, 2002 and Friday June 21, 2002

\begin{tabular}{|c|c|}
\hline Stroke & Neuro-oncology ………................139 to P-152 \\
\hline Dementia & Spinal Surgery …….................... -153 to P-160 \\
\hline 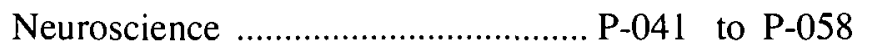 & General Neurosurgery …………….... P-161 to P-175 \\
\hline ... P-059 to P-067 & .... P-176 \\
\hline Epilepsy & Pediatric Neurosurgery \\
\hline General Neurology & Child Neurology ........................... P-194 to P-212 \\
\hline Movement Disorders …................. P-119 to P-129 & Multiple Sclerosis ........................ P-213 to P-218 \\
\hline 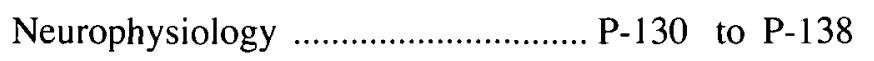 & \\
\hline
\end{tabular}




\section{Society Prize Papers}

\section{Frances McNaughton Memorial Prize - Canadian Neurological SOCIETY}

\begin{abstract}
Selection of acute ischemic stroke patients for intra-arterial thrombolysis with prourokinase using ASPECTS
\end{abstract}

Michael D. Hill (Calgary, Alberta), Howard Rowley (Madison WI, USA), Felix Adler (Boston MA, USA), Michael Eliasziw (Calgary

Alberta), Anthony Furlan (Cleveland OH, USA), Randall

Higashida (San Francisco CA, USA), Lawrence Wechsler (Pittsburgh PA, USA), Carolyn Firszt (Chicago IL, USA), Alastair M. Buchan (Calgary, Alberta) for the PROACT-II Investigators

Background: Selection of acute ischemic stroke patients for thrombolysis is a difficult art, particularly beyond three hours from stroke onset. The baseline computed tomographic (CT) scan may be a useful tool for choosing patients who could particularly benefit from thrombolysis. The Alberta Stroke Program Early CT Score (ASPECTS) is a semi-quantitative method for assessing the baseline CT scan and is more reliable than the "one-third MCA rule".

Methods: We assessed baseline and follow-up CT scans from patients randomized in the PROACT-II study and scored the scans using ASPECTS. The PROACT-II study was a randomized controlled trial of intra-arterial thrombolysis vs. control for patients with middle cerebral artery occlusion. Baseline CT scans were scored using a consensus approach by three experienced observers. Patients were stratified into those with an ASPECT score greater than seven and seven or less. The proportion of patients who achieved an independent functional outcome at 90-days was compared between the two strata according to treatment assignment. Results are reported as relative risks.

Results: One hundred and fifty four patients were included in this analysis. The median baseline ASPECT score was seven (range 210). When patients were stratified into two groups, ASPECTS $>7$ and ASPECTS $\leq 7$, with thrombolysis the relative risk of an independent functional outcome in the high ASPECTS group was 5.0 (95\% CI 1.3-19.2) compared with a RR of $1.0(0.6-1.9)$ in the low ASPECTS group.

Conclusions: Patients with a baseline ASPECT score greater than seven have a five-fold increased chance of an independent functional outcome with intra-arterial prourokinase compared to those with an ASPECT less than or equal to seven. This observation requires confirmation but suggests that ASPECTS can be both a useful clinical tool and an important method of baseline risk stratification in future clinical trials of acute stroke therapy.

\section{The President's Prize - Canadian Association of Child Neurology}

\author{
Long-term neurocognitive outcome following acute \\ disseminated encephalomyelitis in childhood
}

Cecil D. Hahn, Brenda Miles, Daune L. MacGregor, Susan Blaser, Brenda Banwell, Ross Hetherington (Toronto, Ontario)

Background: Cognitive dysfunction has been demonstrated in multiple sclerosis but has not been widely studied following acute disseminated encephalomyelitis (ADEM). Because ADEM often presents with widespread demyelination which may not completely resolve, these patients may be at risk for persistent cognitive dysfunction.

Methods: Children aged 6-15 years admitted between 1995 and 1998 with clinical and MRI features of ADEM were retrospectively identified. Those who consented to participate underwent a structured neurological assessment and neuropsychological evaluation of general cognitive function, processing speed, visuospatial skills, language, memory, and executive function.

Results: Nine of 15 children diagnosed with ADEM met the age criteria and six participated in the study. The mean age at ADEM presentation was $7.7 \mathrm{y}$; the mean duration of follow-up was $3.5 \mathrm{y}$. Group mean scores were within the average range for all cognitive domains examined. Mild impairments in isolated cognitive domains (processing speed, memory, executive functions, attention and planning) were demonstrated in each of the children. In three children, performance IQ (PIQ) was significantly lower than verbal IQ (VIQ), despite normal or minimally impaired motor function.

Conclusions: As a group, these children with prior ADEM functioned within the average range on cognitive testing. However, a variety of mild cognitive deficits were demonstrated on testing, and a cognitive profile of relatively poorer visuospatial function was observed in $4 / 6$ children. These patterns of cognitive deficit are similar to those reported in adult and paediatric multiple sclerosis patients, but are less severe. The milder deficits seen in ADEM may reflect the monophasic nature of demyelination in this disease, compared to the chronic, recurrent demyelination characteristic of multiple sclerosis. 


\section{Society Prize Papers}

\section{The K.G. McKenzie Prize in Clinical Neuroscience Research - Canadian NeUROSURGICAL Society}

\author{
Measuring the health status outcome of children with \\ hydrocephalus
}

A.V. Kulkarni, J.M. Drake, D. Rabin, P.B. Dirks, R.P. Humphreys, J.T. Rutka (Toronto, Ontario)

Background: The measurement of clinical outcome in pediatric hydrocephalus frequently ignores the substantial effect that the condition can have on a child's physical, emotional, cognitive and social health. Therefore, we developed the first health status outcome measure (HSOM) designed specifically for children with hydrocephalus. This was designed as a simple questionnaire to be completed by the children's parents.

Methods: The standardized steps in the development of a HSOM were followed. Item generation involved health professionals and focus groups with parents of children with hydrocephalus. This created a comprehensive list of 187 unique health status items. To reduce this list, questionnaires were sent to 70 sets of parents to assess what they felt were the most important of these health issues. The 53 most important items were then selected to represent the following health domains: physical, social, emotional and cognitive. The 53-item questionnaire was then tested for reliability and construct validity, in another cohort of 75 sets of parents, against the following independent measures of specific components of health: Health Utilities Index (HUI-2), Wide Range Achievement Reading Test (WRAT), Strengths and Difficulties Questionnaires (SDQ), Functional Independence Measure for Children (WeeFIM), and global health ratings by surgeons (GHRS).

Results: The multidimensional 53-item HSOM took approximately 10-15 minutes for the parents to complete and demonstrated excellent test-retest reliability $(0.91,95 \%$ confidence interval (CI) $0.85-0.96)$, inter-rater reliability $(0.87,95 \% \mathrm{CI} 0.76$ 0.93 ), and internal consistency (Cronbach's alpha 0.94). Construct validity was demonstrated by very good Pearson correlations of domain scores with their respective independent measures: HUI-2 (0.81), WRAT (0.58), SDQ (0.70), WeeFIM (0.88), GHRS (0.68).

Conclusions: A HSOM for children with hydrocephalus has been developed and has demonstrated excellent reliability and validity properties. This will provide a valuable measurement tool for a wide range of clinical research projects in pediatric hydrocephalus comparing long-term outcome in different groups of patients.

\section{K.G. McKenzie Prize in Basic Neuroscience Research - Canadian Neurosurgical Society}

Germline mutations of human suppressor of fused predispose to desmoplastic medulloblastoma through activation of sonic hedgehog and Wnt signaling.

Michael D. Taylor, Ling Liu, Corey Raffel, Chi-Chung Hui, Todd Mainprize, Sharon Chiappa, Xiaoyun Zhang, Alisa Goldstein, Theodora Stavrou, Steve Scherer, Wieslaw Dura, Brandon Wainwright, David Hogg, James Rutka (Toronto, Ontario)

Children with Gorlin syndrome and Turcot syndrome are predisposed to develop medulloblastoma due to overactivity of the Sonic Hedgehog and Wnt signaling pathways respectively. We report a novel finding of truncating germline mutations of the Human Suppressor of Fused (HSUFU) gene on chromosome 10q24 in a subset of children with desmoplastic medulloblastoma. Tumour tissue from these children shows loss of heterozygosity at the $H S U F U$ locus in keeping with a classical tumour suppressor gene.

We show that, whereas wild-type HSUFU can block Sonic Hedgehog signaling by binding Gli transcription factors and exporting them from the nucleus, this ability is lost in tumour derived mutants of $H S U F U$. Furthermore, while wild-type $H S U F U$ can block the Wnt signaling pathway by binding its effector Betacatenin and exporting it from the nucleus to the cytoplasm, this ability is lost in tumour derived mutants of HSUFU. We conclude that loss of function of $H S U F U$ in medulloblastoma results in overactivity of both Sonic Hedgehog and Wnt signaling, thereby recapitulating Gorlin syndrome and Turcot syndrome at a molecular level. 


\section{Platform Presentations}

\section{A-01 \\ TUMOUR \\ Germline mutations of human suppressor of fused predispose to desmoplastic medulloblastoma through activation of sonic hedgehog and Wnt signaling. \\ Michael D. Taylor, Ling Liu, Corey Raffel, Chi-Chung Hui, Todd Mainprize, Sharon Chiappa, Xiaoyun Zhang, Alisa Goldstein, \\ Theodora Stavrou, Steve Scherer, Wieslaw Dura, Brandon \\ Wainwright, David Hogg, James Rutka (Toronto, Ontario)}

Winner of the Canadian Neurosurgical Society's K.G. McKenzie Prize in Basic Neuroscience Research. See page 7

\section{A-02}

Prospective trials in the photodynamic therapy (PDT) of malignant brain tumors: The change in Karnofsky score (KS) and neurological examination from preoperative assessment to discharge assessment in recurrent supratentorial malignant gliomas

PJ.Muller, B.C. Wilson, L. Lilge, V. Yang, A. Varma, A. Bogaards (Toronto, Ontario), T. Fullagar, F. Hetzel, Q. Chen (Denver, USA), R. Fenstermaker (Buffalo, USA), R. Selker (Pittsburgh, USA),

J. Abrams (Detroit, USA).

Background: We are conducting two prospective randomized multicentred clinical trials in the PDT of malignant supratentorial malignant gliomas. One of these is designed to determine whether high light dose PDT $\left(120 \mathrm{~J} / \mathrm{cm}^{2}\right)$ is superior to low light dose PDT $\left(40 \mathrm{~J} / \mathrm{cm}^{2}\right)$ in patients with recurrent malignant astrocytic tumors.

Method: We compared the pre-operative KS and elements of the neurological examination (speech function, visual fields, cognitive function, sensory examination and gait) to the postoperative examinations at hospital discharge. The means were compared by paired student-t test.

Results: The KSs 86 of 88 patients with recurrent gliomas were assessable. The mean (SD) preoperative and postoperative KS were $82(14)$ and $79(17)$, respectively $(p=0.003)$. The mean decline in $\mathrm{KS}$, although statistically significant, was small. The median Karnofsky score changed from 90 to 80 . The KS improved in eight patients (postoperative average length of stay (alos) $=9.7$ days), showed no change in 47 (alos $=8.3$ ), declined by 10 points in 24 (alos=13.4) and declined by more than 10 points in 7 (alos=23.3); three of these seven improved in follow-up but did not reach their preoperative KS. The data were not available in 2 .

Conclusion: A small but statistically significant decline in Karnofsky score was identified postoperatively in these recurrent tumor patients. Their hospital average length of stay increased with declining Karnofsky score.

This investigation is supported by grant CA 43892 DHHS/NIH/NCI.
A-03

Dendritic cells pulsed with autologous tumour peptide cause proliferation of cytotoxic $\mathrm{T}$ lymphocytes against glioblastoma multiforme or metastatic melanoma tumour cells in vivo

R.G. Kerr, H. Chen (Edmonton, Alberta); M.P. Protti (Milan, Italy), K.C. Petruk (Edmonton, Alberta)

It is well-known that GBMs and melanomas have evolved complex methods of evading host immune responses. In particular these tumours have very low surface expression of MHC I. Dendritic cells are professional antigen presenting cells which are capable of expressing antigen in the context of MHC I or II and thereby elicit strong and specific $T$ cell responses to presented antigens. We hypothesize that dendritic cells primed in vivo with a naturally processed peptide extract containing a wide range of potentially immunogenic antigens from autologous melanoma or GBM can cause strong cytotoxic $\mathrm{T}$ cell proliferation.

We report our consistent ability to generate dendritic cells from patients' peripheral blood monocytes under the influence of GMCSF and IL-4. We generate cultures of the patients' tumour from O.R. biopsies. We extract from the cytosol of the tumour cells an array of naturally processed peptides, 100-5,000 Daltons in size appropriate for presentation by DCs in the context of MHC. For GBMs ED273bt, and ED326bt, and melanomas ED235mel, and ED335mel we have generated profound autologous CD8+ lymphocyte expansion following cross priming by peptide and cytokine matured DCs. A limited clinical trial employing autologous DCs and naturally processed peptide extract immunotherapy is now underway.

\section{A-04}

Sex steroid hormone exposures and risk for meningioma

B.S. Jhawar, G.A. Colditz, C.S. Fuchs, M.J. Stampfer (Boston, USA)

Objective: To investigate the risk of meningioma in relation to exogenous and endogenous sex hormones.

Research Design and Methods: A prospective cohort of female registered nurses ages 30-55 from 11 US states who completed biennial questionnaires between 1976 and 1996. All participants were free of cancer and major medical illnesses at the onset of the study. The primary endpoint was self-reported meningioma as reported in the biennial and supplemental questionnaires.

Results: During 1,213, 522 person-years of follow-up, 125 cases of meningioma were confirmed. After adjusting for age and bodymass index, compared to postmenopausal women who had never used postmenopausal hormone, the relative risk (RR) for premenopausal women was $2.48(95 \% \mathrm{CI}, 1.29-4.77)$, and for postmenopausal women that were hormone users, the RR was 1.86 (95\% CI, 1.07-3.24). We found no excess risk associated with past hormone use. In models that additionally controlled for hormone use and menopausal status, we found that compared with women whose menarche occurred before age 12, the RR for women with menarche at ages $12-14$ was $1.29(95 \% \mathrm{Cl}, 0.86-1.92)$ and for menarche after age 14, the RR was 1.97 (95\% CI, 1.06-3.66). Although non- 
significant, we also observed a tendency for increased risk of meningioma for parous compared to nulliparous women (multivariate $\mathrm{RR}=2.39,95 \% \mathrm{Cl}, 0.76-7.53)$ ). A trend towards increasing meningioma risk with increasing body-mass index category was also noted ( $\mathrm{P}$ for trend $=0.06$ ). No association was found for past or current use of oral contraceptives.

Conclusions: The risk for meningiomas was increased among women exposed to either endogenous or exogenous sex hormones. An unexpected relationship with increasing age at menarche was also noted that remains unexplained.

\section{A-05}

The importance of a syngeneic glioma implantation model: The F98/Fischer rat model

\section{Mathieu, J.B. Lamarche, D. Fortin (Sherbrooke, Quebec)}

Background: Reviewing current literature on glioma implantation models, one is confronted by a plethora of publications on allogeneic models (e.g. C6 astrocytoma). We hypothesize that the inflammatory reaction raised against the tumour graft in these models is significant to the point of invalidating some therapeutic results obtained. Therefore, a syngeneic model would be optimal in terms of limiting the associated inflammatory reaction.

Method: The F98 glioma cell line was propagated in vitro and initially implanted in Long-Evans rats (allogeneic model). However, less than optimal tumour-take and intense inflammatory reaction prompted us to investigate the use of a syngeneic model (F98/Fischer rat). Therefore, 18 animals of each strain were implanted using the same technique. The animals were sacrificed when symptomatic or after a maximum of thirty days. Tumour-take was evaluated by way of post-mortem microscopic hematoxylin/ eosin examination. Immunohistochemistry was conducted on each sample to detect lymphocytic infiltration.

Results: The tumour take in the syngeneic model (100\%) was superior to the allogeneic model (50\%). Moreover, the extent of the inflammatory reaction was greatly decreased in the Fischer rat model.

Conclusion: We conclude that the use of an allogeneic model for implantation in malignant glioma studies is inappropriate.

\section{A-06}

The educational role of virtual fluoroscopy in transsphenoidal surgery

\section{S.A.Al-Akkad, D. Fewer, K. Kassiri (Winnipeg, MB)}

Background: Teaching the technique of transsphenoidal surgery has always been a challenging experience. The traditional operative layout of transsphenoidal surgery with the C-ARM in position does not allow proper access for more than one person in the operative field. This greatly hinders teaching and learning of the procedure.

Methods: Descriptive study of the new operative layout was presented using pictures. Retrospective analysis was performed using student t-test of 26 teaching cases. These tests compared the technique using the new versus old layout.

Results: Virtual fluoroscopy for transsphenoidal surgery proved to be an efficient teaching and learning method of the transsphenoidal approach. Here we show that this new technique significantly decreased resource time (fluoroscopy machine and technician) ( $\mathrm{p}=0.001)$ in addition to decreasing radiation exposure time $(p=0.03)$. This was without a significant difference in operative time ( $p=0.68$ ), despite the learning curve of the new layout.

Conclusions: The art of performing transsphenoidal surgery has been taught from generation to generation of Neurosurgeons for the last century. Virtual fluoroscopy provides optimal hands-on experience for the residents with a full assistance and direct supervision with significant reduction in radiation hazards and increased overall safety and comfort for the teaching surgeon and the operative team.

\section{A-07}

Osmotic blood-brain barrier disruption to enhance chemotherapy delivery in the treatment of brain tumors: the Sherbrooke experience

A. Desjardins, D. Mathieu, M. Boudrias, D. Fortin (Sherbrooke, Quebec)

Background: The blood-brain barrier (BBB) constitutes an impediment to the delivery of chemotherapeutic agents to the central nervous system. With that knowledge, the concept of transiently "opening" the BBB was developed. A standardized procedure is now in application through an international consortium. Sherbrooke university hospital officially joined this consortium in November 1999. This report details our experience thus far with the procedure.

Method: Demographic, radiological, clinical, quality of life and treatment data were collected prospectively for each patient included in this study. Patients with the following histology were included: malignant astrocytoma, primary CNS lymphoma, metastatic lymphoma, oligodendroglioma, medulloblastoma, brain metastasis, ganglioglioma. Depending on histology, one of two chemotherapy regimens was used: a carboplatin, and a methotrexate-based regimen.

Results: Forty-four patients underwent 187 BBBD procedures. Additionally, 26 patients were submitted to 77 intra-arterial procedures without BBBD because they didn't meet the inclusion criteria. Overall response rate was as follows according to the Macdonald criteria: seven complete responses, 31 partial, 17 stable disease, eight progression, and seven nonassessable disease. Complication rate was $1.5 \%$.

Conclusion: The results are similar to those reported by the "BBB consortium" in "Cancer", February 2000, and emphasise the safety and feasibility of this procedure.

\section{Spinal and Peripheral Nerve}

\section{B-01}

A prospective, randomized, clinical trial comparing anterior versus posterior stabilization for unilateral facet injuries of the cervical spine

\section{Dvorak, B. Kwon, C. Fisher, P. Wing, M. Boyd, (Vancouver,} British Columbia)

Background: Unilateral cervical facet fractures/dislocations may be stabilized by an anterior or posterior approach. This study's purpose was to compare these two techniques in a prospective, randomized fashion. 
Methods: Patients with unilateral facet injuries were randomized to anterior (discectomy, iliac crest bone graft, and anterior cervical locking plate) or posterior (local and/or iliac crest bone graft, and lateral mass plates) stabilization. Postoperatively, the time required to satisfy standardized discharge criteria that reflected independent mobility, medical stability, and acceptable pain control was recorded as the primary outcome measure. Functional and radiographic evaluation was performed three months postoperatively.

Results: In 28 randomized patients, there was no significant difference between anterior and posterior groups, although more variability in time to discharge was observed in the posterior group. Three months postop, there was no significant difference between groups for NASS scores of neurology and pain, although there was a trend towards higher scores in the posterior group.

Conclusion: Patients with unilateral facet injuries treated with either procedure achieve standard discharge criteria in the postoperative period at similar rates. Functional outcome at three months is also comparable. The choice between anterior or posterior procedure may be acceptably based on surgeon preference.

\section{B-02}

Reconstruction of the craniocervical junction with C1-C2 screw fixation: indications, techniques and outcomes in a consecutive series of 51 cases with long-term follow-up

\section{M.G. Fehlings, B. Goulet (Toronto, Ontario)}

Introduction: Transarticular screws are an important option for managing Cl-2 instability, although long-term follow-up on efficacy, safety and application to complex occipitocervical reconstructions is required. We thus present our experience with transarticular screw fixation in 51 consecutive, prospective cases with a mean follow-up of over two years.

Methods: Indications for surgery included trauma $(n=23)$; rheumatoid arthritis $(n=13)$; failed fusion $(n=12)$; degenerative instability $(n=3)$; osteomyelitis $(n=1)$ and os odontoidum $(n=1)$. A Cl-2 fusion alone was performed in 37 cases and a complex multilevel reconstruction in 14 cases. Ninety-eight screws were positioned; four patients had a single $\mathrm{Cl}-2$ screw. All cases were evaluated by lateral flexion-extension films. A postoperative CT with reconstructions was obtained in 31 cases.

Results: There were no neurological or vertebral artery complications. A solid fusion was obtained in 50/51 cases with one asymptomatic pseudarthrosis. By CT criteria, screws were in excellent $(n=20)$, good $(n=17)$, acceptable $(n=14)$ and poor $(n=2)$ position. We found a strong correlation between $>4 \mathrm{~mm}$ of Cl-2 posterior subluxation and non-optimal screw position $(\mathrm{p}<0.001)$.

Conclusions: Transarticular screws represent an important technique to stabilise the $\mathrm{Cl}-2$ joint and with experience can be placed safely. C1-C2 instability with $>4 \mathrm{~mm}$ of non-reducible posterior subluxation is associated with poor fixation and is a relative contraindication for this technique.

\section{B-03}

\section{Multitarget grafting strategies in the lesioned adult rat spinal} cord

\section{S. Christie, I. Mendez (Halifax, Nova Scotia)}

Background: We have previously shown that a commercially available source of human neurons (hNT cells) can survive transplantation into an acute spinal cord injury (SCI) model. The objective of the present study was to investigate whether a multitarget grafting strategy would enhance hNT graft growth and survival in this model.

Materials and Methods: Following a left lateral thoracic hemisection, 54 immunosuppressed, female Wistar rats were randomly allocated into different treatment groups; hemisection only or hemisection and hNT cell transplantation (via a bridge, double or triple graft). Grafting occurred three days after SCI. After thirteen weeks the animals were sacrificed and tissue sections were stained with human neuronal markers.

Results: $66.7 \%$ of the surviving, grafted animals displayed immunohistochemical evidence of graft survival. Fibre outgrowth, greatest in the bridge and triple grafts, was observed in both rostral and caudal directions, at a rate of up to $0.2 \mathrm{~mm} /$ day. Double grafts were smaller, displaying less fibre outgrowth, which did not cross the lesion.

Conclusions: Bridge and triple grafts displayed greater growth and enabled the hNT graft to essentially bridge the lesion. This suggests that hNT neurons have the potential to structurally reconnect the proximal and distal spinal cord across the region of injury.

\section{B-04}

Why do you use methylprednisolone for acute spinal cord injury? A national survey

\section{R. Moulton (Toronto, Ontario), R.J. Hurlbert (Calgary, Alberta)}

Background: Since the publication of NASCIS II in 1990 , methylprednisolone has gained widespread use in the treatment of acute spinal cord injury. We undertook a survey of Canadian spinal cord injury experts to identify the reasons behind methylprednisolone administration.

Methods: In March and June of 2001, specialists attending the Canadian Spine Society and Canadian Congress of Neurological Sciences meetings respectively, were surveyed with respect to the administration of methylprednisolone for acute spinal cord injury. Seven questions were posed in an effort to gain an appreciation of how often the drug was prescribed and for what reasons.

Results: Sixty surgeons responded to the questionnaire who either treat acute spinal cord injuries $(n=58)$ or routinely provide advice to other physicians treating this condition $(n=2)$. Seventy-five percent of the physicians surveyed indicated that they used methylprednisolone to treat acute spinal cord injury. However, only $17 \%$ of those prescribing steroids did so because they felt it was beneficial.

Conclusions: Eighty-three percent of surgeons surveyed are prescribing methylprednisolone in acute spinal cord injury for what might be considered undesirable reasons. These results underscore the need for national organizations to facilitate evidence-based review of specific clinical questions to help direct optimally informed practice patterns and to help curb fears of litigation. 
autografts (positive controls) and significantly better than other

The final strike against steroids: an evidence based review beyond the NASCIS studies

\section{R. John Hurlbert (Calgary, Alberta)}

Background: Over the past decade methylprednisolone has become widely prescribed for the treatment of acute spinal cord injury (SCI). Although it has been proposed as a standard of care, recent challenges have given rise to reconsideration of this tenant. The purpose of this paper is to review the available literature beyond the NASCIS studies and to formulate evidence-based recommendations of the use of methylprednisolone in this setting.

Methods: Through an electronic-data base search strategy, and by cross-reference with published literature, clinical studies investigating the use of methylprednisolone in acute $\mathrm{SCI}$ were identified. They were reviewed in chronological order with respect to study design, outcome measures, results, and conclusions.

Results: Ten studies were identified that attempted to evaluate the role of steroids in non-penetrating (blunt) SCI. Five of these were Class I clinical trials (including NASCIS I, II, and II) while five were Class II studies. All failed to demonstrate improvement due to steroid administration in any of the primary hypotheses testing. While post-hoc analyses were interesting, they failed to demonstrate consistent significant treatment effects.

Conclusions: From an evidence-based approach, methylprednisolone cannot be recommended for routine use in acute nonpenetrating SCI. Prolonged administration of high dose steroids (48 hours) may be harmful to the patient. Until more evidence is forthcoming, methylprednisolone should be considered to have investigational (unproven) status only.

\section{B-06}

Synthetic hydrogel nerve tube enhanced with growth factors for peripheral nerve repair

\section{R. Midha, C.A.Munro, P.D. Dalton, M.S. Shoichet, C.H. Tator (Toronto, Ontario)}

Background: Our long-term goal is repair of peripheral nerve injuries using synthetic nerve guidance devices that improve both regeneration and functional outcome relative to an autograft. Here we report the in vivo application of synthetic hydrogel tubes that are impregnated with growth factors.

Methods: Poly (2-hydroxyethyl methacrylate-co-methyl methacrylate) (PHEMA-MMA) porous tubes, $12 \mathrm{~mm}$ long with an inner diameter of $1.3 \mathrm{~mm}$ and outer diameter of $1.8 \mathrm{~mm}$, were used to repair surgically created $10 \mathrm{~mm}$ gaps in the rat sciatic nerve. The inner lumen of the tubes was filled with collagen matrix alone or matrix supplemented with one of NT-3 at $1.0 \mu \mathrm{g} / \mathrm{ml}$, BDNF at 1.0 $\mu \mathrm{g} / \mathrm{ml}$ or FGF-1 at 1.0 or $10 \mu \mathrm{g} / \mathrm{ml}$. Nerve regeneration through the growth factor enhanced tubes was assessed at eight weeks following repair by histomorphometric analysis at mid-graft level and in the nerve distal to the tube repair.

Results: The tubes were biostable, biocompatible and supported nerve regeneration in over $90 \%$ of cases. Nerve regeneration was improved with the addition of growth factors, as compared both to empty tubes and those containing collagen gel alone (negative controls). Tubes filled with $10 \mu \mathrm{g} / \mathrm{ml}$ of FGF-1 dispersed in collagen demonstrated regenerated nerve cables similar to those observed in samples in terms of the total number of myelinated axons regenerating into the distal nerve $(p<0.01)$.

Conclusions: PHEMA-MMA tubes impregnated with FGF-1 appear to be a promising alternative to autografts for repair of nerve injuries. Studies are in progress to assess the long-term stability of these implants and to further enhance regeneration.

\section{B-07}

Molecular mechanisms of human cervical spondylotic myelopathy: implications for early therapeutic intervention

\section{M.G. Fehlings, W.R. Yu (Toronto, Ontario)}

Although cervical spondylotic myelopathy (CSM) is common, little is known regarding the underlying molecular mechanisms. Based on work in models of neurotrauma, we hypothesized that apoptosis, triggered by activation of the "death receptors" FAS and p75 plays a key role in CSM.

Methods: Sections of post-mortem cervical cord tissue from eight patients with CSM (6M, 2F; age:61-89) and four controls (3F, $1 \mathrm{M}$; age: 43-61) were stained with hematoxylin-eosin/LFB and examined for apoptosis by TUNEL. Double labeling with cell specific markers (GFAP, MAP2, NF200, myelin basic protein and CD68) and co-expression of FAS, p75 and activated caspase- 3 was determined.

Results: Spinal cords from CSM cases were characterized by anterior horn cell loss, chronic inflammation, dorsal root degeneration and extensive loss of myelinated axons. Numerous TUNEL-positive oligodendrocytes (CSM: $90.9 \pm 32.4$; control: $8.2 \pm$ $7.1 ; \mathrm{p}<0.0008$ ) were present in association with degenerating axons. Apoptosis occurred with FAS and p75 death receptor expression and with activation of the effector caspase- 3 .

Conclusions: This is the first demonstration that apoptosis occurs in humans with CSM. A strong association was found between apoptosis, caspase- 3 activation and the death receptors FAS and $\mathrm{p} 75$. Early therapeutic intervention to target $\mathrm{p} 75$ and FAS may attenuate this form of cell death in CSM.

\section{Multiple SClerosis}

\section{C-01}

Secondary progressive MS following a single initial attack: SAP

\section{Kremenchutzky, G.PA. Rice (London, Ontario), G.C. Ebers} (Oxford, UK)

Background: Although usually progression follows a relapsing course, the onset of progressive disease (OPD) is obscure, often camouflaged by overlapping relapses. The clearest separation occurs in subjects appearing to be in transition, having a single attack and progression (SAP). We characterize this subgroup, analyzing its clinical features and long-term outcome, framed on a natural history population-based study.

Methods: Gender, age of onset, symptom (s) involved at MS onset and at OPD, the time to OPD and survival to disability landmarks were compared to other MS subtypes.

Results: We identified 71 SAP cases with age of onset 33.3 years 
and male:female ratio 1:1.84. Mean time from onset to OPD was 7.6 years. Median times from onset to DSS 6,8 and 10 were 12.73, 20.64 and 43.87 years respectively, no different when compared to other MS subgroups ( $p=0.6,0.3$ and 0.3 ). The number of symptoms at onset $(p=0.4)$ and the quality of remission $(p=0.8)$ had no significant effect on outcome.

Conclusions: Characterizing SAPMS allows for better evaluation of the progressive course independently of relapses. Although time to onset of progression appears influenced $a$ priori by early relapse rate (shorter in SAPMS), long-term outcome seems invariable once progression has begun.

\section{C-02}

\section{High intensity immunosuppression with autologous stem cell} transplantation: a treatment for aggressive multiple sclerosis

\section{M.S. Freedman, H.L. Atkins, M. Bowman (Ottawa, Ontario) and the Canadian MS BMT Study Group}

Background: Recent emphasis is placed on early aggressive treatment for MS given that therapies targeting the immune system become less effective over time with the onset of progression due to neurodegeneration as opposed to inflammation. Immunosuppression with cytotoxic agents are partially effective but are limited by toxicity and fail to completely abrogate disease. We postulate that a complete ablation of the immune system might halt ongoing inflammation. Furthermore, transplantation with autologous bone marrow-derived stem cells might, in addition to replacing the immune elements, offer a source of neural precursors to aid in the repair of damaged tissue.

Methods: This tri-city study (Ottawa, Montreal, Toronto) began the summer 2001 and plans to enter 24 patients who will receive the full protocol and eight additional "control" patients who will receive "best medical therapy" but will be followed alongside the treated patients. Patients are chosen on the basis of rapid aggressive early disease and failure to respond to approved disease modifying drugs. Staggered entry is planned to assure safety. Following an initial back-up bone marrow harvest, stem cells are mobilized using G-CSF and cyclophosphamide. Stem cells are collected by leukophoresis and $\mathrm{T}$ cells depleted by magnetic separation of purified CD34+ cells on specialized columns. Immune ablation is accomplished with combination therapy using high dose cyclophosphamide, busulfan and anti-thymocyte globulin. Stem cells are re-infused and expanded in vivo using G-CSF. Clinical and neurological assessments and MRI studies using gadolinium are performed prior to entry, at baseline, between seven and 10 days following the initial cyclophosphamide dosing, and at specified time points over the ensuing three year monitoring period. In depth immunological studies are also being performed at regular intervals, but results will not be discussed here.

Results: The results of the first five patients going through this protocol will be presented. In particular, early safety analyses and MRI studies will be shown.

Conclusions: Although only early follow-up is available, this procedure of complete immune ablation with stem cell rescue for MS appears to be well-tolerated and no sign of active disease can be detected.

\section{C-03}

Inter-caudate distance is a useful linear MRI measure of cerebral atrophy in multiple sclerosis

H. Butzkueven, J. Brown, J. Carey, M. Cook, P. Mitchell, T. Kilpatrick (Melbourne, Victoria); T. Groom, I. Van der Mei, B. Taylor (Hobart, Tasmania)

Background: Measurement of cerebral atrophy in large multiple sclerosis (MS) cohorts poses significant methodological and resource problems. These impede our understanding of the disease processes that may be responsible for progressive atrophy in MS.

Methods: We recruited a defined subset of the MS population of Tasmania. Three putative linear atrophy measures (inter-caudate distance (ICD), frontal horn width and third-ventricular width) were obtained from routine MRI. We determined the most reliable atrophy marker by testing for their ability to differentiate between patients and controls. We then analysed their determinants in controls and finally developed a statistical model to predict degree of atrophy in the Tasmanian population cohort.

Results: 171 patients and 93 controls were analysed. The ICD was the most reliable marker distinguishing MS patients from controls (OR 15.3, $\mathrm{p}=0.001$ ). In normals, ICD was determined by skull size $(p<0.0001)$ and chronological age $(p<0.001)$. In $M S$ patients, ICD was determined by disease duration $(\mathrm{p}<0.001), \mathrm{Tl}$ lesion number $(\mathrm{p}=0.002)$ and male gender $(\mathrm{p}<0.001)$, but not by clinical severity or interferon usage.

Conclusions: The ICD is a valid, reliable measure of atrophy in MS, easily obtainable from routine MRI. We report the novel finding that cerebral atrophy in MS appears worse in males.

\section{C-04}

Guidelines for a standardized MRI protocol for the diagnosis and follow-up of multiple sclerosis

\section{A. Traboulsee, D.K.B. Li, D.W. Paty (Vancouver, British Columbia)}

Background: MRI is very useful because it reveals silent lesions that can help to satisfy the criteria for dissemination in space and time required for diagnosis in MS. However, some clinicians use routine MRI to follow individual MS patients based on clinical trial population data. Furthermore, MRI protocols can vary between or within centers. Therefore, it seemed appropriate to develop a consensus statement on the use of MRI in MS not only for diagnosis but for follow-up.

Methods: An international panel of neurologists and radiologists met in Vancouver, BC on November 3-4, 2001, under the sponsorship of the Consortium of Multiple Sclerosis Centres, to develop guidelines for a standardized MRI protocol for the diagnosis and follow-up of patients with multiple sclerosis.

Results: Development and implementation of standardized MRI protocols for brain and spine were considered useful for the diagnosis and follow-up of patients with MS. MRI with gadolinium should be done as part of the initial evaluation and diagnosis of suspected MS. Routine follow-up MRI is not recommended unless there is suspicion of a secondary diagnosis, unexpected clinical worsening, or prior to initiation of treatment.

Conclusion: The development of these recommendations is only the beginning of what has to be a continuing process, requiring modification, implementation, evaluation and continued updating and improvement. 
Relapsing-remitting MS: new insights into pathogenesis

M.H. Barnett, J.W. Prineas (Sydney, Australia)

Background: Recent work has emphasized the heterogeneity of MS pathology, however little is known of the temporal evolution of newly forming plaque pathology in individuals with the condition.

Methods: Here we describe autopsy findings in a 14-year-old female who presented with neurogenic pulmonary edema and died 16 hours after the onset of her symptoms. She had clinically definite relapsing-remitting multiple sclerosis with three previous attacks, the first of which occurred 11 months prior to the current presentation.

Results: Autopsy revealed a total of 12 plaques. Of these, six were remyelinating shadow plaques and six exhibited active demyelination. The new lesion, considered responsible for the development of neurogenic pulmonary edema, was located in the dorsal medulla and involved the solitary tracts and nuclei. It consisted of broad areas of myelin pallor associated with dramatic oligodendrocyte apoptosis and microglial activation, adjacent to smaller areas of myelin phagocytosis and lymphocytic infiltration.

Conclusion: We describe the pathology of a "pre-phagocytic" phase in the development of the classical relapsing-remitting MS lesion, consisting principally of myelin pallor and marked oligodendrocyte apoptosis. As no similar picture has been observed in classical T-cell mediated EAE nor in antibody-augmented EAE, the present findings suggest that a novel and unexpected pathogenetic mechanism may underlie new lesion formation in relapsing-remitting MS.

\section{C-06}

Evolution of diffuse and focal magnetization transfer abnormalities in multiple sclerosis

C. Laule, I.M. Vavasour, J. Oger, D.K.B. Li, D.W. Paty, A.L. MacKay (Vancouver, British Columbia), D.L. Arnold (Montreal, Quebec)

Background: Magnetization transfer (MT) imaging provides indirect information on tissue structure abnormalities in areas that may appear otherwise normal with conventional magnetic resonance (MRl).

Methods: We determined the evolution of MT in normal appearing white matter (NAWM) and lesions in nine multiple sclerosis (MS) patients and age-matched controls. Serial MT experiments employed a 3D gradient echo with (IMT) and without (InoMT) an MT pulse. Ten NAWM regions of interest were drawn in all subjects and in lesions in MS patients. MT ratio (MTR)= ((InoMT-IMT)/InoMT)*100\%.

Results: Mean NAWM MTR correlated strongly ( $\mathrm{R}=0.93)$ with years since the patient's first attack and was well-characterized by a linear decrease of $-0.16 \%$ year $(\mathrm{p}<0.0001)$. The time zero intercept of the NAWM MTR was $30.6 \pm 0.2 \%$, not different from average control MTR $(30.4 \pm 0.2 \%)$. An additional gradual decrease in NAWM MTR was observed 6-12 months before the appearance of a new lesion on conventional MRI, while a more precipitous MTR decrease was seen 2-6 months before the lesion appeared. Lesions exhibiting a pre-lesion MTR decrease showed less MTR recovery.
Conclusions: MTR of MS NAWM undergoes a slow, progressive decrease that starts at disease onset and accelerates rapidly in focal areas just prior to lesion appearance on conventional MRl.

\section{C-07}

Analysis of the safety and efficacy of cladribine and autologous peripheral blood stem cell transplantation as a treatment paradigm for secondary progressive multiple sclerosis

\section{T.J. Kilpatrick, A. Grigg, P. Shuttleworth, P. Mitchell, J. Szer (Melbourne, Australia)}

Background: Immune ablation and autologous stem cell transplantation (SCT) is a potential treatment for severe MS, assuming regeneration with naïve lymphocytes diminishes autoimmune reactivity. Several protocols are being trialled, each with potentially different therapeutic potential and morbidity.

Methods: We assessed SCT combined with cladribine, attempting to induce selective lymphopenia prior to leukaphoresis. Conditioning was with cyclophosphamide and ATG, prior to autografting of unselected cells.

Results: Four patients with recent onset disease ( $1-5$ years) with EDSS scores between 5.5-7.0, and with a deterioration of $>0.5$ points in the previous six months were enrolled. Three of the patients also deteriorated between enrolment, the administration of cladribine and transplantation. SCT was well-tolerated, although one patient developed serum sickness and two experienced transient neurological exacerbations. Of three patients assessed at 12 months post-engraftment two are stable, whilst the third has improved (EDSS 7.5 to 6.5). Two patients have experienced relapses and are now receiving mitozantrone. MRI data are currently being assessed.

Conclusions: This regimen was well-tolerated and was associated with stabilisation but not inactivation of disease. The benefit of cladribine is questionable, as patients continued to deteriorate prior to transplantation. A comparative analysis of the costs/benefits of SCT versus mitozantrone is required.

\section{EPILEPSY}

\section{D-01}

\section{MRI is useful in the management of a first seizure}

\section{Kilpatrick, A. Lowe, Z. Matkovic (Melbourne, Australia)}

Background: Although the value of MRI in the investigation of chronic epilepsy is established, its role in the assessment of first seizure has not been determined.

Method: This study assessed the use of MRI in 100 consecutive patients presenting to a First Seizure Clinic. Fifteen patients had a non-epileptic seizure, and were excluded. Eighty-five patients had an epileptic seizure (62 tonic-clonic, 8 complex partial, 15 provoked). Eighty-three patients had a CT scan, and 81 an EEG. MRI was performed in 70 patients, 60 in whom CT showed no relevant lesion and 10 patients in whom clarification of CT lesion was needed.

Results: CT revealed a relevant lesion in 17 patients. Six patients with normal CT refused MRI. MRI was abnormal in 12, including four with normal CT (DNET, dysplasia, hippocampal sclerosis, contusion). MRI clarified the nature of the CT lesion in 7, including two CT lesions which were shown to be artefactual. MRI therefore 
provided information additional to CT in $11 / 70(16 \%)$. At six months follow-up $17(20 \%)$ had recurrent seizures. Recurrence was not significantly different in patients with a lesion on imaging (32\% vs $17 \%)$.

Conclusions: We conclude MRI is useful in the management of patients with a first seizure.

\section{D-02}

\section{Event-related fMRI of myoclonic jerks arising from dysplastic} cortex

\section{J.S. Archer, A.B. Waites, P. Federico, G.D. Jackson (Melbourne,} Australia).

Aim: Epileptic myoclonic jerks often have no EEG correlate. We report how event-related functional MRI (ER-fMRI) can localize the origin of myoclonic jerks in the absence of electrographic discharges.

Methods: A 26-year-old female presented with a 19 year history of frequent right foot myoclonic jerks and a normal EEG. Functional MRl at 3 Tesla was performed using two block design trials to map cortical activation during voluntary right and left foot movement. ER-fMRI analysis of right foot jerking was performed on the block design trials and on imaging of spontaneous jerks.

Results: Anatomical MRI demonstrated a focus of dysplasia extending anteriorly from the left parasaggital central sulcus. Block design experiments showed normal activation of contralateral medial primary motor cortex and bilateral supplementary motor cortex. However, voluntary left foot movement also produced increased right foot jerking and activation of the dysplastic cortex. ER-fMRI analysis of the jerks showed activation of the dysplastic, left motor, and supplementary motor cortices, with the dysplastic cortex activating earlier.

Conclusion: These results demonstrate the utility of ER-fMRI for localising the origin of epileptic myoclonic jerks. ER-fMRI also provided temporal information about the brain regions involved in the generation of epileptic activity.

\section{D-03}

\section{Vagal nerve stimulation in children with intractable epilepsy}

M. Connolly, C. Massey, E. Sherman, P. Steinbok, K. Farrell

(Vancouver, British Columbia)

Background: We describe the efficacy and tolerability of vagal nerve stimulation (VNS) in children.

Methods: 26 patients were followed prospectively. Anti-epileptic medication dosages were unchanged during the first 12 months.

Results: 14 males and 12 females, aged 8-18 years, were followed for 6-30 months. The epilepsies included symptomatic localization-related (15), cryptogenic localization-related (5), Lennox-Gastaut (3), and other generalized epilepsies (3). They had been treated with 4-14 anti-epileptic medications, the ketogenic diet (12), and epilepsy surgery (2). A greater than $50 \%$ reduction in seizure frequency was observed in seven of $26(27 \%)$ at six months, in 10 of $23(48 \%)$ at 12 months, and in 3 of $12(25 \%)$ at 18 months. The output current at which optimal benefit occurred was $0.5-2.0$ mAmp (mean $1.5 \mathrm{~mA}$ ). Adverse effects occurred in 11 patients: cough and hoarseness during stimulation $(n=7)$, tingling in neck $(n=3)$, Horner syndrome $(n=1)$ and jerks of the left shoulder during stimulation $(\mathrm{n}=1)$.

Conclusions: Although no patient achieved complete seizure control, $48 \%$ of patients had greater than $50 \%$ reduction in seizures at one year. The benefit of VNS appeared to lessen by 18 months suggesting that tolerance occurred. The relative lack of success and development of tolerance may relate to the population studied.

\section{D-04}

Quality of life, depression, and seizure frequency did not change after one year of vagus nerve simulation

I. Valdivia, J. McGlone, D. Clarke, R. Sadler, M. Penner (Halifax, Nova Scotia).

Background: Vagus nerve stimulation (VNS) has been reported to coincide with improvement in quality of life (QOL) (Cramer, 2001; Dodrill \& Morris, 2001). However, controlled and blinded studies have shown that the magnitude of improvement was similar in treated and non-treated patients. Hence, early improvement may be an artefact (e.g., Hawthorne effect) that disappears by six months (Hoppe et al., 2001).

Methods: Self-reported epilepsy-related QOL (QOLIE-89), nonhealth related QOL (Quality of Life Questionnaire), depressive symptoms (Geriatric Depression Inventory), and seizure frequency were recorded before and one year after VNS in 12 patients. Nine medication controls matched for age (35 years) and reading achievement (low average) underwent the same evaluations 12 months apart.

Results: No VNS patient dropped out. The VNS and medication control groups did not differ in self-ratings on any measure prior to VNS or 12 months later. Within-group comparisons of change showed no statistical difference on QOL measures. Nearly identical means suggest that lack of significance is probably not due to power limitations. Seizure reduction greater than $50 \%$ was achieved in $16.7 \%$ of the patients implanted with VNS.

Conclusions: After one year, QOL was similar in patients implanted with VNS and medication controls. Seizure reduction was also minimal in this prospective, control-matched, non-industry funded study.

\section{D-05}

Gamma oscillations are increased in generalised epilepsies

J.O. Willoughby, S.P. Fitzgibbon, KJ. Pope, L. Mackenzie, A.V. Medvedev, C.R. Clark, M.P. Davey, R.A. Wilcox, (Adelaide, Australia)

Background: Gamma oscillations $(30-100 \mathrm{~Hz}$ gamma EEG activity) correlate with high frequency synchronous rhythmic bursting in assemblies of cerebral neurons participating in aspects of consciousness. Previous studies in a kainic acid animal model of epilepsy revealed increased intensity of gamma rhythms in background EEG preceding epileptiform discharges, leading us to test for intensified gamma EEG in humans with epilepsy.

Methods: We recorded 64 channel cortical EEG from 10 people with primary generalised epilepsy, 11 with acquired (partial) epilepsy and 20 normals during a quiescent mental state. Using standard methods of EEG analysis we quantified the strength of 
EEG rhythms (fast Fourier transformation) and compared the strengths of rhythms in the patient groups with normals by unpaired $t$ test at $1 \mathrm{~Hz}$ intervals from $1-100 \mathrm{~Hz}$.

Results: In patients with generalised epilepsy, there was a 3-7fold increase in power of gamma EEG between 30 and $100 \mathrm{~Hz}(\mathrm{P}<$ $0.01)$. Analysis of three unmedicated patients with primary generalized epilepsy revealed an additional 10 -fold narrow-band increase of power around $35-40 \mathrm{~Hz}(\mathrm{P}<0.0001)$. There were no corresponding changes in patients with partial epilepsy.

Conclusions: Increased gamma EEG is likely to be a marker of the underlying ion-channel or neurotransmitter receptor dysfunction in primary generalised epilepsies and may also be a pathophysiological prerequisite for the development of seizures. The finding provides a new diagnostic approach and also links the pathophysiology of generalised epilepsies to emerging concepts of neuronal correlates of consciousness.

\section{D-06}

I123-4-Iododexetamide single photon emission tomography (SPECT) in temporal lobe epilepsy

A. Mohamed, S. Eberl, M. Kassiou, S. Beveridge, C. Constable,

D. Henderson, MJ. Fulham (Camperdown, Australia)

Background: 1123-4-lododexetamide (Idex) is a muscarinic acetylcholine SPECT ligand. We present Idex SPECT findings in a group of 11 patients with medically refractory temporal lobe epilepsy (TLE).

Methods: We performed interictal Idex SPECT scans at 0, 3, 6 and 24 hours post injection in a group of 11 patients with medically refractory TLE. The patients underwent extensive testing including surface video EEG (11), invasive EEG (2), volumetric MR (11), FDG PET (11), ictal HMPAO SPECT (3) and neuropsychological testing (11). five patients had a temporal lobectomy. A blinded observer interpreted all imaging and EEG studies. Statistical region of interest (ROI) analysis was performed on the 6 hour Idex SPECT studies.

Results: Using EEG/surgery as standard for localization of seizure onset, the concordance of the imaging studies were: MR (7/11), FDG PET (8/11) and visual review of Idex SPECT (11/11). The ROI analysis of the Idex SPECT was concordant in 11/Il patients. Interestingly in two patients where there was discordance between MR, PET or surface EEG findings, Idex SPECT was correct in lateralizing seizure onset when invasive EEG recordings were performed.

Conclusions: Preliminary results suggest that interictal Idex SPECT is an excellent localizing tool in TLE.

\section{D-07}

Functional MRI spike localization to the left superior temporal gyrus with Wernicke's area transfer in a patient with Rasmussen's encephalitis

P.Federico, J.S. Archer, I.E. Scheffer, A.S. Harvey, R.M. Wellard, G.D. Jackson (Melbourne, Australia).

We studied a 10-year-old boy with Rasmussen's encephalitis and a two year history of complex partial seizures with auditory, dysphasic, and autonomic symptomatology. Video-EEG telemetry,
MRI, PET, SPECT, and neuropsychological testing localized the major abnormality to the left superior temporal gyrus (STG) with normal language.

Three Tesla functional MRI of language was performed before and after surgery. Spike localization with fMRI-EEG was performed twice. Subdural electrodes were implanted.

Spike-triggered $\mathrm{FMRI}$ of five spikes in each study showed a small focus of activation in the left posterior STG, corresponding to Wernicke's area (WA). Intracranial video-EEG telemetry confirmed this localization and, of all investigations, fMRI-EEG best predicted this intracranial localisation. Language fMRI showed normal activation in the left Broca's area; no activation in the left WA, but activation in the right posterior STG. Direct cortical stimulation of the left WA failed to produce a language disturbance despite provocation of seizures, suggesting no critical language function. Cortical resection of the left STG caused no language disturbance. Postoperative language fMRI confirmed a right WA.

In conclusion, we have shown that with spikes originating in an abnormal left STG, WA can transfer to the right hemisphere while Broca's area remains in the left.

\section{Basic Neuroscience/NeURology}

\section{E-01}

Differential activation of Broca's area by lexical and semantic language tasks: an fMRI study

D. Blacker (Rochester, MN, USA), G. Thickbroom, M. Byrnes, F. Mastaglia (Perth, Australia)

Background: Silent language tasks are associated with activation of frontal lobe areas including Broca's, however the relative role of these areas in language processing is not fully understood. The objective was to compare patterns of activation in the frontal lobe during lexical and semantic language processing.

Methods: Functional MRI (8 right handed subjects) studied two silent semantic language tasks using visually presented word lists of animal names: (i) association of animal actions, (ii) animal descriptions, and a lexical task consisting of recalling animal names starting with a specified letter of the alphabet.

Results: Activation was observed in Broca's area, the dorsolateral prefrontal cortex (DLPFC), and the supplementary motor area (SMA). There were no significant differences in activation of DLPFC or SMA across tasks, however Broca's area activation was approximately doubled during semantic tasks compared with the lexical task (actions vs names: 19.1 vs 8.9 voxels, $p=0.02$; descriptions vs names 24.4 vs 10.1 voxels, $\mathrm{p}=0.04$ )

Conclusions: Broca's area is activated during single word recall, but is more active during tasks that have semantic content, while preparatory processing areas (SMA) and memory retrieval (DLPFC) are engaged equally. This has implications for fMRI language testing paradigms targeting frontal lobe language areas. 


\section{E-02}

\section{Nitric oxide up-regulation after chronic brain ischemia precedes visuo-spatial impairment in rats}

J.C. de la Torre (San Diego, USA), B.A. Pappas, (Ottawa, Ontario), V. Prevot, (Portland, USA), G.B. Stefano (New York, USA)

One of the primary roles of released nitric oxide (NO) from capillary endothelial cells is to inhibit platelet and leukocyte adhesion to the endothelium while maintaining normal blood fluidity and local cerebral blood flow. To test whether chronic brain ischemia (CBI) can upset the homeostatic balance of released NO in the hippocampus and thereby affect visuo-spatial memory in rats, we subjected 12-month-old Sprague-Dawley rats to either bilateral common carotid artery occlusion for two or eight weeks $(\mathrm{N}=16)$ or to no-occlusion controls ( $\mathrm{N}=20$ ). Rats were tested in the Morris water maze two and eight weeks after sham-occlusion or CBI and NO was measured following water maze testing. For NO determination, rats were decapitated and $2 \mathrm{~mm}$ plugs were quickly dissected from the hippocampus, parietal cortex and median eminence. Tissue plugs were placed in a buffer solution and NO levels were measured amperometrically using a nanosensor microelectrode. This technique senses nNOS and eNOS release at the nanomole range from tissue and microvessels. Results showed that abnormal NO up-regulation occurred prior to visuo-spatial memory deficits in rats with $\mathrm{CBI}$ at either two or eight weeks when compared to no-occlusion controls. In rats with visuo-spatial deficits after CBI, NO up-regulation was similar at two and eight weeks to CBI rats without memory deficits. NO up-regulation with or without visuo-spatial deficits was present mainly in the hippocampus not in cortex or median eminence and appeared higher at eight than at two weeks following CBI. Controls showed no up-regulation of NO and no visuo-spatial deficits at any time period. The data suggest that NO up-regulation precedes visuo-spatial deficits at two weeks and continues to rise by eight weeks. Increased NO release from tissue or endothelium may be a compensatory reaction to $\mathrm{CBI}$, particularly in the hippocampus, a region linked to memory and learning and may be an important clue to the pathogenesis of Alzheimer's disease.

\section{E-03}

Evidence for axon loss in the retinal nerve fiber layer in patients with recovered optic neuritis

\section{F. Costello, R. Kardon, A. Lee, M. Wall, (Iowa City, USA)}

Purpose: To assess the prevalence of retinal ganglion cell loss in patients with previous optic neuritis who have recovered visual function and to correlate this with visual field threshold sensitivity.

Methods: 12 patients with previous optic neuritis were studied at least two months following the acute event (follow-up median 12 months, range 2-150 months). Optical Coherence Tomography (OCT) was performed on both eyes using a $1.73 \mathrm{~mm}$ radius circular scan of the peripapillary retina (retina surrounding the optic nerve head) and the retinal nerve fiber layer thickness was determined. Each patient also had visual field testing performed (Humphrey automated threshold test 24-2) and a neuro-ophthalmologic evaluation. Information was also recorded about the acute event, neurologic status, and MRI scan of the brain.

Results: 11 of the 12 patients with recovered optic neuritis showed some degree of thinning of the retinal nerve fiber layer compared to the normal fellow eye, or compared to normal subjects. In six patients, an automated visual field test was available from the acute phase of the optic neuritis, as well as from the recovered phase. In these six patients (12 eyes) there was a highly significant correlation (correlation coefficient $r=0.665, P=0.018$ ) between the initial extent of visual field damage (decibel mean deviation of threshold sensitivity from normal) and the permanent loss of axons in the nerve fiber layer at recovery.

Conclusions: Axon loss in the retinal nerve fiber layer was common in the patients studied who had recovered optic neuritis. The degree of axon loss was correlated with the severity of the initial visual field loss.

\section{E-04}

Coeliac disease - associated neurological and respiratory complications

\section{S. Gopinath, S. Hodgkinson, G. Marks (Liverpool, Australia)}

Background: In 1888, Samuel Gee gave an accurate description of the condition we call coeliac disease. Since 1965, neurological and respiratory disorders with coeliac disease have been described. We present a case of coeliac disease with an uncommon neurological and respiratory presentation.

Merhods: The case: A 44-year-old woman presented with progressive gait deterioration. Five years prior, she had developed a right hand dominant tremor. Over the following two years she developed a severe productive cough, reduced exercise tolerance and weight loss. She gradually deteriorated with truncal and gait ataxia, spastic paraparesis and wasting of hands. She also developed an acute left abducent nerve palsy.

Result: Blood investigations showed negative antigliadin and endomysial antibodies, elevated cardiolipin antibodies. Her blood film showed Howell Jolly bodies and thrombocytopenia consistent with hyposplenism. Small bowel biopsy showed villous atrophy. MRI of her brain and spine and cerebrospinal fluid studies were normal. Bronchial biopsies showed mild active inflammation and moderate subepithelial basement membrane thickening.

She failed to respond to a gluten free diet. She was managed with oral steroids, cyclophosphamide and clarithromycin.

Conclusions: There is a wide spectrum of neurological disorders in coeliac disease. The response to immunosuppression and antibiotics will be discussed.

\section{E-05}

Neuroimaging and frontal subcortical dysfunction in the Sydney longitudinal study of stroke

J.C.L.Looi, P.S. Sachdev, H. Brodaty, MJ. Valenzuela, W. Wen, L. Lorentz, J. Sims, D. Gillies, R. Shnier (Sydney, Australia)

Aims: To assess structural-functional correlations between MRI and neuropsychological function in a large Australian cohort of stroke patients. To explore the contribution of cortico-striato-pallidothalamic-cortical circuits to cognitive dysfunction post-stroke.

Methods: 100 stroke patients recruited from stroke units of three major Sydney teaching hospital and 80 healthy elderly controls underwent detailed neuropsychological testing (attention, speed, conceptual, executive, language, memory and premorbid function) 
and MRI (T1,T2, FLAIR) neuroimaging three months after their stroke. The MRI data were stored on acquisition digitally reconstructed (3D) and analyzed (via ANALYZE program) for atrophy, infarctions and hyperintensities using a standardised rating scale. The volumes of infarction were described and measured using a semiautomated method.

Results: Preliminary analysis showed executive dysfunction and slowed information processing, which was not strongly correlated with either stroke volume or hyperintensity load, but generalized atrophy. Detailed analysis is proceeding to determine the contribution of frontal atrophy and subcortical hyperintensities in frontal-subcortical regions.

Conc/usions: Frontal executive neuropsychological dysfunction has been demonstrated and correlates with generalized MRI atrophy. There may also be a contribution from fronto-subcortical circuit dysfunction.

\section{E-06}

Performance of normal elderly subjects in cognitive test-retest

\section{A. Garcia, K. Pullman, A. Boruvka, L. Evans (Kingston, Ontario)}

$M$. Freedman (Toronto, Ontario)

Background: Elderly subjects might require repeated cognitive testing. Test-retest learning effects might result on improved scores.

Methods: We investigated the effect of test-retest on the California verbal learning test 1 (CVLT), Mattis Dementia rating scale (DRS) and the Stroop scores, in 172 elderly, communitydwelling subjects. They were tested at baseline and two years after. Paired T-tests were used to compare differences between the scores obtained at baseline and repeat testing.

Results: Subjects mean age was $72.6(\mathrm{SD}=4.64)$ years and had a MMSE of $28.40(S D=1.54)$ at baseline. Time interval between tests was 126.5 ( $\mathrm{SD}=33.9)$ weeks. MMSE score at second visit was 28.27 $(S D=1.55)$. Subjects had 13.43 ( $S D=3.12)$ years of education. They performed significantly ivorse in short-delay, long-delay and cued recall, intrusions, discriminability, false positives and response bias of the CVLT and the Stroop test, at the second visit, compared to baseline. However, learning scores (semantic, serial) and DRS scores were unchanged (but for the sub-score of memory).

Conclusion: The results suggest that in normal elderly subjects, recall measures worsen with time in spite of equal learning ability. Re-testing in normal elderly subjects does not result in a learning effect, falsely improving recall scores.

\section{NEUROPHYSIOLOGY}

\section{F.01}

Experimental atypical absence seizures: early and late-stage cholesterol inhibition and epileptogenesis

\section{Serbanescu, MA. Cortez, S.C. Cumnane, O.C. Snead III (Toronto, Ontario)}

Background: The EEG, behavior, pharmacology and developmental characteristics of the AY-9944 (AY) model are similar to those of malignant pediatric atypical absence epilepsy. (Neurology 2001; 56: 341-349). Whether the developmental inhibition with other late-stage or early-stage cholesterol inhibitors produce comparable seizure effects to those seen in the AY model, remains to be determined (Epilepsia 1999; 40 (7):40).

Methods: Long Evans Hooded rats were treated with AY (7.5 $\mathrm{mg} / \mathrm{kg})$ or BM $15.766(\mathrm{BM})(20 \mathrm{mg} / \mathrm{kg})$ or U $18,666 \mathrm{~A}$ (U 18$)(10$ $\mathrm{mg} / \mathrm{kg})$ or lovastatin $(100 \mathrm{mg} / \mathrm{kg})$ or equivalent volume of saline every six days from postnatal day $(\mathrm{P}) 2$ to $\mathrm{P} 20,(\mathrm{~N}=6$, each group). EEG recordings were performed at P55 for spike-and-wave discharge (SWD) quantification. Brains were removed for sterol measurements.

Results: Recurrent SWD duration (mean seconds/hour \pm SD) did not differ between AY (391.8 \pm 311.8$)$, the U18 (258.8 \pm 68.3$)$ the BM (145.8 \pm 164.97$)$ and the lovastatin groups $(389 \pm 172)$. All groups had higher SWD duration than the control group $(25.56 \pm 47.7)(p=$ 0.001 Student's $\mathbf{t}$ Test).

Conclusion: Data indicate that early and late-stage inhibition of cholesterol synthesis during rat brain development produce chronic experimental atypical absence seizures.

\section{F-02}

\section{Bone conducted vestibular-evoked myogenic potentials (BVEMPs)}

\section{M.S. Welgampola, S.M. Rosengren, G.M. Halmagvi,} J.G. Colebatch, (Sydney, Australia)

Background: Vestibular-evoked myogenic potentials (VEMPs) to intense clicks and tones, recorded from the sternomastoid muscles (SCM) are of saccular origin and require integrity of the middle ear conductive apparatus

Methods: We recorded BVEMPs to $112 \mathrm{~dB} / 7 \mathrm{~ms} / 250-2000 \mathrm{~Hz}$ tones delivered via a B7I clinical bone vibrator over the mastoid process in 10 normal subjects, six with conductive hearing loss and four postvestibular neurectomy. VEMPs to $145 \mathrm{~dB}$ (SPL) clicks and $124 \mathrm{~dB}$ (SPL) tones delivered via headphones were measured for comparison. Biphasic plnl responses were measured peak to peak and corrected for background EMG. The optimum stimulus location was mapped and reflex thresholds were measured.

Results: Biphasic plnI responses were recorded from both SCM. Ipsilateral responses occurred earlier and were larger. Average pl and $\mathrm{nl}$ latencies were $13.6 \pm 1.8 \mathrm{~ms}$ and $22.3 \pm 1.2 \mathrm{~ms}$. BVEMPs were frequency selective and had broad tuning curves with optimum stimulus frequencies at 250,500 or $1000 \mathrm{~Hz}$. VEMPs to airconducted clicks and tones were absent in subjects with conductive hearing loss; BVEMPs were preserved. BVEMPs were absent following vestibular neurectomy. Stimuli delivered posterior and superior to the external acoustic meatus yielded the largest responses. Average thresholds for BVEMPs, air-conducted tones and clicks were $100.3+5.0,114 \pm 5.3$ and $131.7 \pm 4.9 \mathrm{~dB}$ (SPL).

Conclusion: BVEMPs are a potentially useful measure of vestibular function especially in the presence of conductive deafness.

\section{F-03}

\section{Beta-amyloid-induced decrease in the intracellular calcium} level

Andras Palotas, Janos Kalman, Miklos Palotas, Anna Juhas-, Zoltan Janka, Botond Penke (Szeged, Hungary)

The ubiquitously present beta-amyloid peptide plays an 
important role in the pathogenesis of Alzheimer's disease. Mounting evidence suggests alterations of various biochemical processes of cells of peripheral tissues in this form of dementia. In the course of surveying a wide variety of these modified metabolic pathways, we examined the intracellular free calcium levels of lymphocytes and fibroblasts harvested from sporadic Alzheimer patients and from age-matched controls. Calcium concentration was measured in Fura2AM-loaded human cells by dual wavelength spectrofluorimetry. Alzheimer fibroblasts exhibited lower basal calcium level as compared to the control cultures. The resting calcium concentration appeared to be higher in Alzheimer lymphocytes when compared to that seen with control cells. Exposure to beta-amyloid resulted in increased calcium concentration of the control lymphocytes and fibroblasts. Alzheimer cells, on the other hand, have proved to be resistant to beta-amyloid. Chronic exposition of fibroblasts to the peptide, however, caused a fall in the calcium-level. Apparently, beta-amyloid has dual effects: acutely, it increases the calciumconcentration of cells; in contrast, on the long-run, beta-amyloid peptide acts as a calcium- antagonist.

\section{F-04}

The EMG findings in the anterior compartment syndrome, a case presentation

\section{Q. Ghanem (Ottawa, Ontario)}

The Anterior Compartment Syndrome of the lower extremity is now a very rare condition, hardly seen by electromyographers. In 25 years of practicing electromyography, I had not seen it. The most senior orthopedic surgeon at our hospital cannot recall seeing one in the past 15 years. This is a function of the good standard of medicine practiced in Canada.

The clinical findings are very similar to the relatively common peroneal nerve entrapment neuropathy, and to L5 radiculopathy. The EMG findings are quite different, however.

This presentation will demonstrate with the help of videotape the unusual combination of normal EMG in peroneus longus, normal sensory nerve conduction in the superficial peroneal nerve, marked denervation in extensor digitorum brevis, and the bizarre and complete silence of a necrosed tibialis anterior during attempted activation of the muscle, as well as during insertion.

\section{F-05}

Intraoperative recording of motor nerve action potentials from the brachial plexus after transcranial electrical stimulation

\section{D.A. Houlden, M. Vennettilli, R. Midha, (Toronto, Ontario)}

Background: Ventral nerve root integrity is essential for beneficial nerve grafting within the brachial plexus. Motor nerve action potentials (MNAPs), recorded intraoperatively from the brachial plexus after transcranial electric stimulation (TCES), may be useful in assessing ventral nerve root function.

Methods: Intraoperative TCES (Digitimer D185 stimulator) was performed using a train of three stimuli with a $1.6 \mathrm{msec}$ interstimulus interval in four patients. MNAPs were obtained from hook electrodes placed on the upper, middle and lower trunks of the exposed brachial plexus. Responses from selected muscles in the upper limbs were concomitantly obtained. Studies were performed before and after the administration of muscle relaxants in one patient.
Results: MNAPs were recorded from all trunks of the brachial plexus. Threshold stimulus intensity for MNAPs and muscle responses were similar. MNAP and muscle response amplitude increased as TCES intensity increased. Muscle relaxants abolished the muscle responses but did not change MNAP amplitude or latency.

Conclusions: Intraoperative TCES using a short train of stimuli was effective in evoking MNAPs from all trunks of the brachial plexus. TCES is a promising tool for intraoperative evaluation of ventral nerve root function during reconstructive brachial plexus surgery.

\section{F-06}

\section{Cold induced sweating: a report of two cases}

J.G.McLeod (Sydney, Australia); A. Hahn (London, Ontario); R.R. Tuck, P.M. Satchell (Sydney, Australia)

Background: Two cases of cold-induced sweating are described and the pathophysiology of this condition has been investigated. A similar case was reported by Sohar et al (Sohar et al (1978) Lancet, ii, 1073-4).

Method: Both cases have been investigated with autonomic function and electrophysiological studies, sural nerve and skin biopsies.

Result: One case was a Canadian female aged 24 and the other an Australian male aged 46 . There was no family history in either case. They had dysmorphic features and a history of sweating induced by cold but not by heat. Lowering body temperature induced sweating over the face, arms and trunk but not lower limbs. Slight sweating occurred on lower limbs and groins with elevated temperatures. Tests of autonomic function demonstrated normal cardiovascular reflexes, postganglionic sympathetic efferent function, unmyelinated fibres on nerve biopsies and sweat glands on skin biopsies. Symptoms were controlled by the $\alpha 2$-adrenergic agonist clonidine.

Conclusion: This rare congenital condition of paradoxical sweating induced by cold is caused by a specific central impairment of sudomotor function.

\section{Pediatric Neurosurgery}

\section{G-01}

Measuring the health status outcome of children with hydrocephalus

A.V. Kulkarni, J.M. Drake, D. Rabin, P.B. Dirks, R.P. Humphreys, J.T. Rutka (Toronto, Ontario)

Winner of the Canadian Neurosurgical Society's K.G. McKenzie Prize in Clinical Neuroscience Research. See page 7

\section{G-02}

Functional performance following selective posterior rhizotomy: long-term results using a validated evaluative measure

S. Mittal, J.P. Farmer, B. Al-Atassi, K. Montpetit, N. Gervais, C. Poulin, M.A. Cantin, T.E. Benaroch (Montreal, Quebec)

Background: Selective posterior rhizotomy may result in considerable benefit for children with spastic cerebral palsy. The 
authors analyzed data obtained from the McGill Rhizotomy Database to determine the long-term functional performance outcome of dorsal rhizotomy.

Methods: A multidisciplinary team evaluated children preoperatively, at six months, and one year postoperatively. Quantitative standardized assessments of activities of daily living (ADLs) using the Pediatric Evaluation of Disability Inventory (PEDI) measure were obtained. Of 57 patients who met the entry criteria for the study, 41 and 30 completed the three-year and fiveyear assessments, respectively.

Results: Statistical analysis demonstrated significant improvement in mobility and self-care domains of the functional skills dimension at one year after SPR. The preoperative, 1-, 3-, and 5 -year scaled scores for the mobility domain of the functional skills were $56.0,64.0,77.2,77.8$, respectively. The scaled score for the self-care domain increased from 59.0 presurgery to $67.9,81.6$, and 82.4 at the 1-, 3-, and 5-year assessments, respectively.

Conclusions: Significant improvements in functional performance, using the PEDI evaluative measure, are seen 1-year after SPR. The improvements persist at 3- and 5-year follow-up. We conclude that SPR using intraoperative stimulation is valuable in augmenting motor function and self-care skills essential in performance of ADLs.

\section{$\mathbf{G - 0 3}$}

Mutism after posterior fossa tumor resection in children: incomplete recovery on long-term follow-up

P. Steinbok, D.D. Cochrane; R. Perrin, A. Price (Vancouver, British Columbia)

Background: Mutism after posterior fossa tumor resection is generally said to be transient. Our experience suggested that speech did not usually normalize, and that mutism was associated with neurologic deficits that did not recover fully.

Methods: Children with mutism after posterior fossa tumor resection, and alive more than two years postoperatively, were reviewed retrospectively. Charts were reviewed and parents contacted to ascertain details about mutism, associated neurologic deficits, and the most recent speech and neurologic status.

Results: There were six children, with follow-up ranging from 2.5 - 13.1 years (mean 7.4 years). Tumors were midline, with four astrocytomas and two medulloblastomas. Mutism was noted immediately after postoperative extubation in all patients. Speech reappeared $1-14$ weeks postoperatively, except for one patient, who remained mute at 2.5 years. Speech returned to normal in only one patient. Mutism was always accompanied by new or worsened cerebellar ataxia, which resolved incompletely in the long-term. Sixth nerve palsies occurred in three, and recovered incompletely. Seventh nerve paresis occurred in two and recovered completely.

Conclusion: Mutism after posterior fossa tumor resection is associated with other neurologic deficits, particularly ataxia. Whereas speech usually returns, contrary to general opinion, speech rarely normalizes. Other associated deficits rarely resolve completely.

\section{G-04}

Complications of invasive subdural grid monitoring in children undergoing epilepsy surgery

James T. Rutka, C. Onal, H. Otsubo, S. Chitoku, A. Ochi, S. Weiss, O.C. Snead III (Toronto, Ontario)

We reviewed our experience at The Hospital for Sick Children with complications associated with invasive subdural grid monitoring between $1996-2001$. Thirty-five consecutive children (17 males and 18 females with a mean age of 11.7 years) with intractable epilepsy were studied. The mean duration of epilepsy before surgery for the group was 8.3 years. Fifteen children (43\%) had a history of at least one neurosurgical intervention either diagnostic or therapeutic prior to invasive monitoring. Adverse events were categorized as either neurological or surgical. Unilateral motor weakness and dysphasia were the most prominent postoperative neurological adverse events. No child had a permanent hemiparesis despite a high incidence of transient postoperative weakness $(69 \%)$. One child had exacerbation of a pre-operative dysphasia which was permanent. Thirteen children had visual field defects which were anticipated based on the areas of planned cortical resections. Hypertrophic scar, CSF leak, and phlebitis were the most common surgical adverse events with occurrences of $26 \%, 20 \%$, and $20 \%$, respectively. Five children (14\%) demonstrated temporary postoperative worsening in seizure control. Non-symptomatic postoperative hemorrhagic complications including subdural, intracerebral, and epidural hematomas were observed radiographically in $31 \%$ of patients. Four male patients $(11 \%)$ demonstrated significant postoperative behavioural alterations with one showing schizophrenic symptoms. Three patients $(9 \%)$ had infections at the operative site with two superficial wound infections, and one osteomyelitis. Blood transfusions were required in $80 \%$ of the entire group with a trend towards less blood loss in those children whose grids had less than 100 contact points. Overall, $83 \%$ of the children benefited from surgery. Our study suggests that carefully selected pediatric patients with intractable epilepsy benefit from invasive monitoring with acceptable but definite risks which should be disclosed to the patient and families.

\section{G-05}

Comparing diagnostic times between adult and pediatric brain tumor cohorts within the Maritimes

\section{Mehta (Edmonton, Alberta), A. Chapman, L. Clarke (Halifax.} Nova Scotia)

Background: The latency from symptom onset to diagnosis of pediatric brain tumors within the Maritimes has previously been described. Within Canada, the time from symptom onset to diagnosis between an adult and pediatric brain tumor cohort within a captive geographical location has never been described.

Methods: We compared data from both our pediatric and adult brain tumor databases. Data from 1995-2001 within our pediatric database and 2001 within our adult database were compared.

Results: Approximately 200 patients were reviewed within both databases. Among the pediatric cohort, the mean time from symptom onset to diagnosis was 29.2 weeks (95\% CI 20-38 weeks). Pediatric brainstem tumors took longer to diagnose than those 
outside the brainstem, 46 weeks (95\% CI, 12-80 weeks) versus 26 weeks (95\% CI, 16-36 weeks). Only $41 \%$ of pediatric patients were correctly diagnosed within three visits to a physician. Within the adult cohort, the mean time from symptom onset to diagnosis was 18 weeks and 73 weeks for patients harboring malignant gliomas and meningiomas respectively $(p<0.05)$. Sixty-six percent of the adult patients were diagnosed within three visits to physician.

Conclusions: Within the Maritimes, adult primary malignant brain tumors have the least delay between symptom onset to diagnosis followed by pediatric intra-axial tumors and adult meningioma patients.

\section{G-06}

Ventricular shunting for hydrocephalus in children: patients, procedures, surgeons and institutions, 1989-2000

\section{D.D. Cochrane (Vancouver, British Columbia) , J.R. Kestle (Salt}

Lake City, USA)

Introduction and Purpose: Ventricular shunting remains the principle and most generally applicable method to treat hydrocephalus in children. This paper describes the demographics of this treatment in English Canada during the period of 1989 to 2000.

Methods: Hospital discharge records were obtained for patients less than 17 years. A database was constructed relating patients to hospital discharges based on scrambled patient identifiers, year of birth, sex, postal code and diagnoses.

Observations: 5947 patients underwent ventricular shunting procedures for hydrocephalus in this period. 265 surgeons working in 73 institutions provided 12110 interventions (shunt insertions: ventriculoperitoneal -5009 , ventriculoatrial -119 , ventriculopleural - 28. Revisions: 6951). Infection was deemed to have occurred in 1059 of 12110 procedures.

Over the study period, the median number of procedures per surgeon was four with $75 \%$ of surgeons performing fewer than 24 procedures. The mean surgeon infection rate was $10 \%$ (SD 20.1). The average number of procedures per year for treating hospitals was 16 with range of one to 233 and SD 33. Mean institutional infection rate was 11.4 (SD 23, median -6.0).

Conclusions: Monitoring of procedure and infection rates in hydrocephalic children is possible at an interprovincial level. As the majority of surgeons treating children with hydrocephalus provide surgical services infrequently, outcome monitoring should be based on institutional, regional or provincial patient populations.

\section{MOVEMENT Disorders}

\section{H-01}

Thalamic deep brain stimulation for the treatment of tremor due to multiple sclerosis: a prospective study of tremor and quality of life

C.R. Honey, C. Berk, J. Carr, M. Sinden, J. Martzke, (Vancouver, British Columbia)

Background: Thalamic deep brain stimulation (DBS) can improve the tremor of multiple sclerosis (MS). This study prospectively followed a cohort of twelve patients to determine if it also improved their quality of life.
Methods: Videotapes of the patients' tremor were taken preoperatively and two and 12 months postoperatively and scored by a neurologist blinded to the treatment. Patients were tested pre- and postoperatively to measure any changes in their reported ability to perform selected activities of daily living and in their health-related quality of life. Patients were also asked to complete a questionnaire relating to their satisfaction with surgery.

Results: There were significant reductions in postural, action and overall tremor at two and 12 months postoperatively. The patients' reported ability to feed themselves was significantly improved at two months $(p=0.01)$. There were short-term trends towards improvement in reported dressing ability, personal hygiene, and writing. There were no significant changes in the SF-36 subscales or total score. Patient satisfaction with the procedure was variable.

Conclusions: In this cohort of MS patients with tremor, thalamic DBS significantly improved their tremor and ability to feed themselves. Which MS tremor patients can benefit the most from this procedure will be discussed.

\section{H-02}

Is the target for thalamic DBS the same as for thalamotomy?

Z.H.T. Kiss, M. Wilkinson, J. Krcek, O. Sucherowsky, W. Murphy (Calgary, Alberta), B. Hu (Ottawa, Ontario), R.R. Tasker (Toronto, Ontario)

Background: Deep brain stimulation (DBS) has virtually replaced thalamotomy for the treatment of tremor. Whereas the optimal site for lesion-making is well-known, the site for thalamicDBS has not previously been investigated. The aim of this study was to determine whether the site used for thalamic-DBS matches the site at which thalamotomy would be performed.

Methods: Ten patients who underwent detailed microelectrode recording and stimulation for placement of DBS electrodes were followed for 12-48 months. An experienced surgeon, blinded to outcome and final electrode position, selected the ideal thalamotomy site based on the reconstructed maps obtained intra-operatively. The site of long-term clinically-used DBS and theoretical thalamotomy location was calculated in 3-dimensional space and compared for each of the $x, y$, and $z$ axes.

Results: There was no significant difference in the medio-lateral location of DBS and theoretical lesion site. However the DBS electrodes were located significantly more anterior $(\mathrm{p}=0.006)$ and dorsal $(\mathrm{p}<0.001)$ to the ideal thalamotomy target.

Conclusion: Whereas it is commonly thought that the site for thalamic DBS is the same as that for thalamotomy, the active DBS electrodes are anterior and dorsal to this location. This mismatch may reflect a requirement for the active pole to be farther away from the postero-ventrally located sensory nucleus, to minimize paraesthetic side effects, or it may provide clues to the underlying mechanisms of effect of DBS and thalamotomy.

\section{H-03}

The organization of the pallidofugal fiber system in primate

M. Parent, M. Lévesque, A. Parent (Beauport, Quebec)

Background: The current model of the organization of the pallidofugal fiber system assumes that axons emerging from the lateral half of the internal pallidum (GPi) course ventrally in the ansa 
lenticularis (AL), whereas those arising from the medial half of GPi run dorsally along the lenticular fasciculus (LF).

Methods: We investigated this organization by single-axon tracing methods in cynomolgus monkeys and the axonal arborization of 70 neurons was reconstructed in 3-D.

Results: Limbic and motor neurons were identified in primate $\mathrm{GPi}$. Limbic neurons occur peripherally; their axon courses exclusively in LF and arborizes densely in lateral habenula. The more abundant motor neurons occupy the entire core of GPi but, irrespective of the location of their cell body, their highly branched axons exit either through $\mathrm{AL}$ or $\mathrm{LF}$, or from medial pole of GPi, inbetween $\mathrm{AL}$ and LF. The GPi motor axons arborize within thalamic motor nuclei, thalamic centre median/parafascicular complex and brainstem pedunculopontine tegmental nucleus.

Conclusions: These findings reveal that the location of cell bodies within the GPi is not a good predictor of where pallidal axons emerge. They call for a reevaluation of the dual (AL/LF) concept of the pallidofugal projections, which guides current stereotaxic surgery for Parkinson's disease.

\section{H-04}

\section{Essential tremor evolution - a clinicopathological study}

Alex Rajput, Chris Robinson, Ali H. Rajput (Saskatoon, Saskatchewan)

Background: Essential tremor (ET) is the most common cause of pathological tremor. There is no report on long-term follow-up of autopsy verified ET.

Methods: 21 ET patients followed by the same neurologist over a 32 year period who came to autopsy were included. ET was diagnosed with postural/kinetic tremor involving the upper limbs (UL) and/or head with no other cause identified.

Results: 21 (M-10, F-11) patients had median onset at 55 (childhood-68) years and median death at $78(50-92)$ years. Tremor onset was UL in 14, UL + head in five and head only in two cases. Of the $14 \mathrm{UL}$ onset cases, five later developed head tremor. One head tremor onset subsequently developed UL tremor. Resting tremor (RT) without bradykinesia or rigidity was noted in seven cases. No consistent pathology was noted in the ET. Parkinson syndrome (PS) was diagnosed in six cases including drug-induced parkinsonism (2) and autopsy diagnoses of progressive supranuclear palsy (2), idiopathic Parkinson's disease (1), and basal ganglia status lacunaris (1).

Conclusions: Tremor becomes more widespread with time in ET. RT was noted in one-third and PS was diagnosed in almost one-third of ET cases. Further studies are required to determine the longitudinal course of ET in the general population.

\section{H-05}

Quantified fast nonlinear deformation for functional neurosurgical planning and analysis

Y.P. Starreveld, K.W. Finnis, D.G. Gobbi, A.G. Parrent, T.M. Peters (London, Ontario)

Background: The analysis and planning of functional neurosurgical procedures is facilitated via nonlinear registration of deep brain structures with standard atlases. Current nonlinear registration algorithms are too slow and error-prone, making clinical application unrealistic.

Methods: An algorithm was developed that deforms a source image to match a target image by maximizing a multi-scale normalized cross-correlation similarity metric. A map of this metric monitors registration accuracy. Testing was performed using linearly and nonlinearly deformed solid models, and on the recovery of known nonlinear deformations applied to Tl-iveighted MRI brain images.

Results: Linear and non-linear deformations of spherical solid models, were recovered to a volume difference of $<1 \%$. The errormap is sensitive to mis-registrations of $0.5 \mathrm{~mm}$. An average brain of 78 different subjects nonlinearly registered to a standard brain demonstrates very high signal-to-noise in the deep brain nuclei and tracts. Average computation time for the nonlinear registration of a T1-weighted MRI brain volume is 10 minutes (dual $933 \mathrm{MHz}$ PIII).

Conclusions: The improvements in the quality, speed and quantification of the registration presented make it possible to contemplate planning and intra-operative guidance of procedures using this technique.

\section{H-06}

Clinical and PET evaluation of subthalamic deep brain stimulation for Parkinson's disease: effects of low and high frequency stimulation

\section{A.F. Sadikot, A.P. Strafella, M. Panisset, A. Dagher, (Montreal, Quebec)}

Background: The glutamatergic subthalamic nucleus (STN) plays a major role in the control of movement. Recently, highfrequency deep brain stimulation (DBS) of the STN has been identified as a potentially important therapy for Parkinson's disease (PD). The mechanism of action of STN DBS remains unclear.

Methods: We studied the clinical response and changes in cerebral blood flow (CBF) resulting from different frequencies of unilateral or bilateral STN DBS in six patients with advanced PD.

Results: Unilateral STN stimulation contralateral to the most affected side decreased UPDRS motor scores ("off medication state") by $17.0 \%$ at $60 \mathrm{~Hz}(\mathrm{p}<0.001)$, and by $34.0 \%$ at $185 \mathrm{~Hz}(\mathrm{p}<$ $0.001)$. Bilateral STN high-frequency stimulation decreased the UPDRS motor score by $48 \%$ ( $p<0.0001)$. Changes in CBF in response to different frequencies of DBS were determined using positron emission tomography (PET), while patients performed paced self-selected movements using a joy-stick. Increasing recruitment of frontal lobe areas associated with planning occurred on progression from unilateral low frequency, to unilateral high frequency, and then bilateral high frequency DBS.

Conclusions: Depolarization block achieved at high frequency results in improved function of frontal areas involved in motor planning and is a major mechanism of salutary effects of STN DBS in PD. 


\section{Child Neurology}

I-01

\section{Long-term neurocognitive outcome following acute disseminated encephalomyelitis in childhood}

Cecil D. Hahn, Brenda Miles, Daune L. MacGregor, Susan Blaser, Brenda Banwell, Ross Hetherington (Toronto, Ontario)

Winner of the Canadian Association of Child Neurology's President's Prize - see page 6.

\section{I-02}

\section{Prognostic significance of failure of the initial antiepileptic} drug in children with absence epilepsy

\section{E. Wirrell (Calgary, Alberta), C. Camfield, P. Camfield, J. Dooley} (Halifax, Nova Scotia)

Background: In children with childhood absence epilepsy (CAE) and juvenile absence epilepsy (JAE), to determine the impact of failure of the initial antiepileptic drug (AED) for lack of efficacy on eventual seizure control and long-term remission of epilepsy.

Methods: Centralized EEG records for the province of Nova Scotia allowed identification of all children seen with CAE or JAE between 1977 and 1985. Information regarding success or failure of initial AED in fully controlling seizures and long-term seizure control and remission of epilepsy was collected by patient questionnaire and chart review.

Results: Eighty-six of 92 eligible patients were followed up (75 CAE, $11 \mathrm{JAE})$. Initial AED treatment was successful in $52(60 \%)$ of 86. Success tended to be greater for valproate (VPA) than for other AEDs ( $p=0.07$ ), and lower if generalized tonic-clonic or myoclonic seizures coexisted $(p<0.004$ and $p<0.03)$. Terminal remission was more likely if the initial AED was successful than if it had failed ( $69 \%$ vs $41 \%$; p $<0.02)$. Compared with those in whom the initial AED was successful, subjects whose initial AED had failed were more likely to progress to juvenile myoclonic epilepsy (JME) at last follow-up (32\% vs $10 \% ; \mathrm{p}<0.02)$ and to develop intractable epilepsy (17\% vs $2 \%$; p<0.04).

Conclusions: Initial AED was successful in $60 \%$ of children with AE. If the first AED failed, the outcome was less favorable, with a lower rate of terminal remission and a higher rate of progression to JME and intractable epilepsy.

\section{$\mathbf{I - 0 3}$}

Limb-girdle muscular dystrophies: focus on a pediatric population

H. Kolski (Edmonton, Alberta), J. Vajsar, B. Banwell, D. Biggar, V. Jay, B. Alman, L. Becker (Toronto, Ontario)

Background: This clinico-pathological correlative study explores the spectrum of previously unclassified pediatric patients with limbgirdle muscular dystrophy.

Methods: The study population consists of patients with acquired limb-girdle distribution weakness presenting to the Hospital for Sick Children, Toronto in 1987-2000, inclusive. Previous muscle biopsy specimens were analyzed, with specific staining performed for the sarcoglycanopathies (alpha, beta, gamma, and delta), merosin, and immunoblots for calpain 3. Dystrophinopathies were excluded.

Results: Overall, we identified 19 suitable patients. Upon further processing of their respective muscle specimens, the overall diagnostic yield was $8 / 19(42 \%)$. In this exclusively pediatric cohort, the most common finding was that of primary gamma sarcoglycanopathy (4 children), followed by calpainopathy (3), and alpha sarcoglycanopathy (1).

The gamma sarcoglycanopathy patients were amongst the most severely affected children. They presented with progressive weakness by $3-5$ years of age and two were wheelchair dependent by 11 years of age. The calpainopathy patients presented later, 6-14 years of age, with a less rapidly progressive course.

Conclusions: This study highlights the importance of considering gamma sarcoglycanopathy in children with severe muscular dystrophy presentations. Protein analysis techniques, such as these, assist in uncovering new and specific diagnoses, have implications for genetic counseling, and provide guidance for molecular biology testing.

\section{I-04}

Increased brain damage following hypoxic-ischemic induced neonatal seizures correlates with enhanced release of excitatory amino-acids

Jerome Y. Yager , Edward A. Armstrong (Saskatoon, Saskatchewan); Elaine C. Wirrell (Calgary, Alberta)

Background: In a previous study, we found that although prolonged seizures per se did not cause brain damage, they worsened the damage resulting from mild hypoxia-ischemia $(\mathrm{HI})$ in the 10-day old newborn rat brain. In the current study we correlate the release of excitatory amino acids with the extent of brain injury in the newborn rat pup.

Methods: Ten-day old rat pups were anesthetized ( $1 \%$ halothane), and microdialysis probes were stereotactically placed in the CA2-3 region of the hippocampus. Kainic acid (KA) was used to induce seizures. Three groups were identified: Group I - received KA alone; Group II - 30 minutes of $\mathrm{HI}$ alone; and Group III - 30 minutes HI plus KA. Hypoxia-ischemia was induced by ligating the right common carotid artery and exposing the rat pups to 30 minutes of $8 \%$ oxygen:balance nitrogen. Seizures were induced by subcutaneous ( $\mathrm{sc}$ ) injection of $3 \mathrm{mg} / \mathrm{kg} \mathrm{KA}$, followed by a $2 \mathrm{mg} / \mathrm{kg}$ sc infusion for three hours. Previous studies had shown that this protocol resulted in continuous electrographic and clinical seizures for a mean duration of 4 hours 42 minutes. Microdialysis samples of alanine, gaba-amino-butyric acid (GABA), glutamate, glutamine, serine and taurine were obtained prior to treatment (control), following $\mathrm{HI}$, prior to KA injection, and at 1 (onset of seizures), 3 , 5, 7 (terminus of seizures), and 9 hours following KA. Significance was determined at $\mathrm{p}<0.05$.

Results: There was a significantly higher mortality in those animals receiving KA (Groups I and III), vs those that did not. During HI (Groups II and III), glutamate, glycine, alanine, taurine, and GABA rose significantly above control. In those rat pups who received KA (Group I and III), glutamate, glycine, taurine and GABA rose significantly above control during the seizures. Concentrations of glutamate were significantly higher in those animals receiving $\mathrm{HI}$ and $\mathrm{KA}$ compared to those receiving either $\mathrm{HI}$ alone or $\mathrm{KA}$ alone for the duration of the seizures, and four hours of recovery. 
Conclusion: The release of glutamate is enhanced during seizures in the newborn brain which follow a mild hypoxic-ischemic event, and may be one of the underlying mechanisms for the exacerbation of brain damage seen in our model of neonatal seizures. Glutamate antagonists may play a specific role in protecting the brain under these circumstances. (Supported by the Toronto Hospital for Sick Kids Foundation)

\section{I-05}

Frontal lobe epilepsy in childhood

D.B. Sinclair, M. Wheatley, K. Aronyk, T. Snyder, P. Smyth, D. Gross, J. McKean, M. Berg (Edmonton, Alberta).

Background: Frontal lobe epilepsy (FLE) is poorly understood. We sought to better characterize FLE in childhood.

Methods: We reviewed pediatric patients admitted to the Comprehensive Epilepsy Program at the University of Alberta Hospitals with a proven diagnosis of FLE. All patients underwent long-term video EEG monitoring, neuropsychological assessment, and had an MRI. Age, seizure, semiology, EEG, MRI, and treatment outcome was examined.

Results: Twenty-one patients, 13 males and 8 females, were studied. Age of onset was variable from 10 months to 16 years (mean 7.5 years). A family history of epilepsy was seen in nine patients. Seizures were brief ( 30 seconds -2 minutes), stereotypic, nocturnal (17/21), and frequent (3-22/night). Clinical features included explosive onset, screaming, agitation, stiffening, kicking or bicycling of the legs, and incontinence. The diagnosis of FLE was not made in any child before referral. The referring diagnosis was sleep disturbance (9), psychiatric problems (6), or other seizure types (6). Interictal EEG was usually normal (17/21). Long-term video EEG monitoring demonstrated frontal (9) or bifrontal (12) epileptic discharges. MRI was abnormal in most patients (17/2I). Seizure control was difficult with only half $(10 / 21)$ the patients being controlled on medication. Three intractable patients went on to epilepsy surgery and became seizure free.

Conclusions: FLE in childhood is a distinct epilepsy syndrome with characteristic features independent of etiology or age. The seizures are brief, stereotypic, nocturnal, and frequent. EEG and MRI are often normal. The condition is often misdiagnosed as a sleep disorder or psychiatric problem. Seizures are difficult-to-control, but may respond to carbamazepine, valproic acid, or epilepsy surgery.

\section{I-06}

Behaviour and adaptive functioning on long-term follow-up of non-accidental head injury

K.M. Barlow (Vancouver, British Columbia); E. Thomson, D.L. Johnson, R.A. Minns (Edinburgh, UK)

Background: There is little detailed information regarding the outcome of non-accidental head injury. This study reports the behavioral difficulties and adaptive functioning in children with non-accidental head injury (NAHI).

Methods: Entry criteria: all children registered on the NAHI database at the Royal Hospital for Sick Children, Edinburgh UK. As part of a longitudinal cohort outcome study, neurological examinations and neuropsychological assessments were performed.

Results: 25 cases consented. The median age at follow-up was
4.8 years. Using the Glasgow Outcome Score, 11 cases were classified as "good", 5 "moderately disabled" and 9 "severely disabled". Self-injurious behaviors were reported in six and eight had severe temper tantrums. Problems with attention (4), hyperactivity (3), impulsivity (5) and sleep disturbance (6) were also seen in older children. The median score on the Behavior Scale of the Bayley Scales of Infant Development where applicable was 8 th percentile (range: 1 to 54$)(n=15)$. The median percentiles on the Vineland Adaptive Behavior Scales were: Communication: 8 (range: $0.1-92$ ), Daily Living: 25 (range: $0.5-53$ ), Socialization: 25 (range: $0.1-61$ ), Motor: 8 (range: 0.1-73).

Conclusions: Behavioral abnormalities are common sequelae of $\mathrm{NAHI}$ and frequently develop towards the end of the second year. Neuropsychological analysis demonstrates a large range in adaptive behavior scores.

\section{I-07}

\section{Primary generalized epilepsy presenting as atypical migraine} in children

\section{P. Sinyth, D.B. Sinclair (Edmonton, Alberta)}

Background: Children with migraine frequently have atypical presentations. An overlap between migraine and epilepsy has been welldescribed in the literature. There are several reports of Benign Occipital Epilepsy of Childhood (BOEC) presenting as atypical migraine. We describe nine cases of primary generalized epilepsy in a pediatric population whose presentations mimic atypical migraine symptoms.

Methods: Retrospective Chart Review of nine patients presenting to Pediatric Epilepsy Clinic.

Results: Nine children presented to our clinic with migraine-like spells. These consisted of brief spells of nausea, vomiting, paleness, lethargy, and confusion \pm headaches. All nine patients were eventually diagnosed with primary generalized epilepsy with the aid of ictal electroencephalographic recordings and history. Four children had photosensitive generalized epilepsy, and one patient experienced migrainous symptoms triggered by bright lights. Our patients all had strong family histories for migraine and/or epilepsy. The children were healthy otherwise with normal imaging and physical examinations. All had excellent response to valproic acid with disappearance of events following treatment.

Conclusions: We report nine cases of primary generalized epilepsy presenting as atypical migraine. EEG recordings during the attack were consistent with primary generalized epilepsy. To our knowledge, the presentation of primary generalized epilepsy with migraine-like features has not been reported in the literature as a case series. Awareness of this unusual condition is essential for early detection and treatment.

\section{I-08}

Aspirin therapy for the prevention of recurrent cerebral thrombo-embolic events in children: a prospective cohort study

\section{R. Hung, G. deVeber, Anthony Chan, (Toronto, Ontario)}

Background: Clinical trials have shown ASA to be effective and safe in preventing recurrent cerebral thromboembolic events (CTE) in adults. However, the safety, tolerability and failure rate of ASA in children with stroke has not been assessed.

Methods: Consecutively identified children ( 1 month to 18 years 
age) with arterial ischemic stroke or transient ischemic attack treated with ASA at the Hospital of Sick Children from January 1992 to May $200 \mathrm{I}$ were assessed for safety, tolerability, and failure of ASA in the prevention of recurrent CTEs.

Results: Eighty-six children were studied. Age ranged from 1.5 months to 16 years (mean 6.4 years) and $59 \%$ were male. Mean ASA dose was $3.93 \mathrm{mg} / \mathrm{kg} /$ day ( 1.3 to $16 \mathrm{mg} / \mathrm{kg} /$ day). Stroke risk factors included vasculopathy $(50 \%)$, prothrombotic / hematologic $(30 \%)$, and cardiac conditions (26\%). Recurrent CTEs occurred in $17 \%$ of patients. ASA side effects, in $22 \%$ of patients, included minor bleeding $(5 \%)$, one major bleed, dyspepsia (12\%) and bruising $(9 \%)$. No patients developed Reye's Syndrome.

Conclusion: ASA is safe and well-tolerated in pediatric patients ivith CTEs. However, ASA failed to prevent recurrent CTEs in $17 \%$. A randomized controlled trial is needed to assess alternative therapies for the prevention of recurrent CTEs in children.

\section{GENERAL NEUROSURGERY}

\section{J-01}

\section{Surgical experience with ventrally located foramen magnum meningiomas}

\section{F. Durity (Vancouver, British Columbia)}

Background: Ventrally located foramen magnum meningiomas are uncommon $(\mathrm{n}=8$ over a 12 year experience, 1990 to 2001 inclusive, in a skull base practice greater than 200 patients). Prior to the mid 1980s, complete removal was uncommon and serious neurological mortality and morbidity exceeded 50 per cent.

Methods: A far lateral transcondylar extended skull base approach was adopted to attain a more complete tumor removal with reduced tissue manipulation and yet to achieve improved outcomes.

Results: Patient ages ranged from $37-65$ years. Sex: seven females: one male. Presenting signs and symptoms included incidental finding $(n=1)$, isolated severe neck pain $(n=1)$, multiple lower cranial neuropathies $(n=s i x)$, mild central nervous system deficit $(n=1)$ and advanced tetraparesis $(n=1)$. Tumors were widely based on the ventral foramen dura and all were associated with upper cervical cord and medullary tissue compression, with (n $=7$ ), or without encasement of the lower cranial nerves, or distortion and encasement of the vertebral artery $(n=6)$. Seven tumors were totally removed except the dural base, and in one instance a small flat plaque was left. Four patients acquired temporary new or worsened lower cranial nerve deficits and two developed worsened central deficits with one permanent. All achieved independence (Glasgow Outcome Score 1 or 2) but two did not return to work.

Conclusion: Ventral foramen magnum meningiomas, although difficult, are amenable to surgical resection with good functional outcomes utilizing advanced skull base techniques.

\section{$\mathrm{J}-\mathbf{0 2}$}

Combined fronto-temporo-orbito-zygomatic osteotomy and optic canal decompression in the treatment of unilateral compressive optic neuropathy due to mass lesions in skull base

\section{C.B.Agbi, S. Gilberg, D. Zackon, (Ottawa, Ontario)}

Background: Optic nerve compression from predominantly unilateral lesions in the skull base results in progressive unilateral visual loss and, if untreated, to ocular blindness. The goals of treatment for these lesions is twofold: firstly to preserve/restore visual function, and secondarily complete removal of the lesion. It is difficult to achieve both goals with a standard craniotomy alone because of the limited access this provides and the inability to mobilize the optic nerve and avoid manipulation injury during surgery. A fronto-temporo-orbito-zygomatic (FTOZ) osteotomy provides a more complete exposure of these lesions allowing more robust removal. Complete optic canal bony and ligamentous decompression allows its mobilization during surgery, reducing surgical manipulation.

Methods: We present our experience in nine cases of optic nerve compression treated by a combined FTOZ osteotomy and complete optic canal decompression and optic nerve mobilization, followed by extirpation of the mass lesion. All patients received pre- and postoperative neuro-ophthalmologic evaluation.

Results: We obtained postoperative improvement in visual function in six cases, and stabilization in three.

Conclusion: We believe that complete exposure of the anatomy through a FTOZ osteotomy and intracanalicular decompression and mobilization of the optic nerve offers the best surgical approach for treatment of these lesions

\section{$\mathbf{J}-\mathbf{0 3}$}

A prospective pilot study of outpatient stereotactic brain lesion biopsy

\section{R.D. Bhardwai, M. Bernstein (Toronto, Ontario)}

Background: To assess the safety and feasibility of performing computed tomography (CT)-guided stereotactic brain biopsy as an outpatient day surgery procedure.

Methods: From 1996, a prospective trial of outpatient stereotactic biopsy was initiated. The protocol consisted of pre-admission education of the patient, CT-guided biopsy under local anesthesia using the Brown-Roberts-Wells or Cosman-Roberts-Wells frame, postoperative observation in the Post Anesthetic Care Unit for two hours and in the Day Surgery Unit for two hours, and then being discharged home four hours after the procedure.

Results: 76 patients constitute the intent-to-treat group of whom two were unable to be discharged on the same day $(97.4 \%$ success rate). The two patients were converted to inpatients because: one needed to be admitted for intravenous antibiotics for a brain abscess, and the other patient had a hard lesion in the brainstem that precluded biopsy needle penetration and admission for observation was elected. Two patients experienced complications (2.6\%): one small intraventricular hemorrhage producing mild headache only, and one case of mild worsening of pre-existing leg weakness with a negative CT.

Conclusions: Outpatient stereotactic brain biopsy is a safe treatment option, which besides having cost savings, may have other hidden benefits.

\section{$J-04$}

Patient-centered disclosure: The Windsor experience

S. Brien, S. Sriharan, S. Chakravarthi (Windsor, Ontario)

Background: Nearly 1200 surgical patients were potentially exposed to Creutzfelt-Jakob disease (CJD) by instrument contamination. A process of disclosure was developed with the 
assistance of the CMPA to notify the at-risk patients.

Methods: Our process of disclosure began just prior to the press release. Ninety-four neurosurgical patients were identified. The attending surgeons contacted all neurosurgical patients. This contact took place within 48 hours from the news release. Patients came to the office the following week for debriefing. Families of patients who had expired were included in this disclosure process. A form letter with all of the needed information was reviewed with them. They were informed that a similar letter would go to the family doctor. Patients were asked directly what their reaction to disclosure was and this was recorded. Patients were seen again according to their planned follow-up and followed for a year.

Results: Ninety percent of the patients stated that they supported our decision as a surgical group for full disclosure. Few patients required additional assistance following the disclosure. Patients, however, were concerned about how they were to be treated if they needed further surgery. Families and patients felt that it was their right to access confidential patient information concerning the indexed patient.

Conclusions: Nearly 100 patients were advised by their neurosurgeons of the potential risk of exposure to CJD. Consistent information delivered in a timely fashion and disclosure by a person of trust was important to patients during this disclosure process.

\section{$\mathrm{J}-05$}

Third ventriculostomy for the treatment of hydrocephalus: can Shunts be removed?

\section{W. Hader, J. Silvaggio, T. Myles, M. Hamilton (Calgary, Alberta)}

Background: Third ventriculostomy is a safe and effective treatment for hydrocephalus in selected patients. We compared the outcome and complications of third ventriculostomy between patients with newly diagnosed hydrocephalus and those with previous shunting procedures.

Methods: A retrospective review of patients undergoing third ventriculostomy, from 1996-2001, at Alberta's Children's Hospital and Foothills Medical Centre was completed. Patient data obtained included: clinical presentation, diagnosis, age at initial shunt, number of revisions, age at third ventriculostomy, technique, complications, follow-up and subsequent shunting procedures performed.

Results: A total of 59 patients were identified. 28 (78\%) patients undergoing primary third ventriculostomy and $17(74 \%)$ previously shunted patients were shunt-free at last follow-up. Serious complications occurred in seven $(30 \%)$ previously shunted patients compared to three $(8 \%)$ patients undergoing primary $\mathrm{TV}$. Complications included meningitis in four, cranial nerve palsy in three, lacunar infarct in one, and epidural hematoma requiring craniotomy in one patient.

Conclusions: Third ventriculostomy is an effective treatment in selected patients with newly diagnosed hydrocephalus as well as those patients with a previous shunting procedure presenting with malfunction. Serious complications with TV occur more commonly in previously shunted patients than in patients treated for newly diagnosed hydrocephalus.
$\mathrm{J}-06$

Treatment of chronic pain by intrathecal drug therapy versus conventional pain therapies: a cost effective analysis

K. Kumar, G. Hunter, D. Demeria, S. Malik, (Regina,

Saskatchewan)

Background: There is limited research data available measuring the cost-effectiveness of intrathecal drug therapy (IDT) compared to conventional pain therapy (CPT). This study tabulates actual dollar costs of consecutive series of IDT patients compared to a control group.

Method: We present a series of 67 patients suffering from failed back syndrome, of which 23 underwent implantation of a programmable drug delivery pump, while 44 patients acted as controls. We followed these patients for a five-year period and tabulated the actual costs incurred in investigations, professional fees, implantation including hardware, nursing visits, alternative therapies, and hospitalization for breakthrough pain. Cumulative costs for each group were calculated for a five-year period. The Oswestry questionnaire was utilized to assess impact of treatment on quality of life.

Results: The actual cumulative costs for IDT over a five-year period were $\$ 29,410$, versus $\$ 38,029$ for CPT. High initial costs of equipment required for IDT are recovered by 28 months. After this point, managing patients using CPT became the more expensive treatment option for the remainder of the follow-up period. The Oswestry Index showed a $27 \%$ improvement for the IDT group, as compared to $12 \%$ improvement in controls.

Conclusion: IPT is cost-effective in the long-term despite high initial costs of implantable devices.

\section{J-07}

The effectiveness of an injury prevention educational intervention in teaching young hockey players about concussion

\section{M.D. Cusimano, DJ. Cook, C. Tator, E. Mansfield, D. Ullrich} (Toronto, Ontario)

Background: Concussion accounts for $12 \%$ of injuries amongst minor hockey league participants in Ontario. Educational intervention is proposed to reduce concussion in minor hockey. A video, Smart Hockey, has been produced by Think First of Canada. It includes information on the mechanism of concussion, in-game tactics to reduce high-risk manoeuvres, and guidelines for leaving and returning to play. A multiple choice and written answer format pre/post test of concussion knowledge was administered to Atom aged players (10-11 years old) before and after viewing Smart Hockey.

Methods: Atom 'A' hockey players $(\mathrm{N}=17)$ were recruited through league officials and given the concussion knowledge test. Smart Hockey was presented to the players. The test was repeated. Tests were scored using a standardized answer key. The change in mean scores was tested using a paired t-test.

Results: The players test scores increased by $48 \% \quad(\mathrm{P}<.01)$ following the video. $53 \%$ of players' test scores increased following the video, $47 \%$ remained the same and no player scored lower following the video. Both knowledge of the mechanism of injury and post-injury symptoms increased after viewing the video.

Conclusion: The Smart Hockey video is effective in increasing 
knowledge of concussion among Atom 'A' hockey players in the short term.

\section{$\mathrm{J}-08$}

\section{Cardiovascular risk factors for physician-diagnosed lumbar} disc herniation

B.S. Jhawar, G.A. Coldit, C.S. Fuchs, M.J. Stampfer, (Boston, USA)

Objective: Recent evidence suggests that degenerative disc disease may be associated with atherosclerotic vascular disease. We therefore explored whether cardiovascular risk factors were associated with a higher incidence of lumbar disc herniation.

Research Design and Methods: We prospectively examined the association between smoking, high cholesterol, high blood pressure, obesity and diabetes and self-reported lumbar disc herniation. The study was conducted among 98,325 female nurses who in 1976 were ages 30-55 years, free of cancer (except non-melanoma skin cancer), lumbar disc disease, and who responded to the 1992 questionnaire regarding whether or not a physician diagnosis of lumbar disc herniation had been made.

Results: During 16 years of follow-up ( 441,748 person-years), 2,734 nurses who were free of cancer reported a physiciandiagnosed lumbar disc herniation that was confirmed either by $\mathrm{CT}$ or MRI. After adjusting for age, body-mass index, smoking, vigorous or moderate exercise, employment status, having seen a physician in the last year, and each of the following other factors, the multivariate relative risk for diabetes was 1.51 (95\% confidence interval (CI), 1.16 to 1.96$)$; for hypertension it was $1.24(95 \% \mathrm{CI}, 1.10$ to 1.40$)$; for high cholesterol it was $1.25(95 \% \mathrm{Cl}, 1.09$ to 1.44$)$, and for having a parent that had suffered a myocardial infarction before age 60 it was 1.15 (95\% Cl, 1.04 to 1.28 ). In these models, compared to never smokers, the relative risk for past smokers was $1.10(95 \% \mathrm{Cl}$, 1.00 to 1.20 ), for current smokers the risk increased with the number of cigarettes smoked per day. A decrease in risk occurred following cessation. Also in these models, we noted a significant trend of increasing risk with higher body-mass index $(\mathrm{p}=0.01)$.

Conclusions: Cardiovascular risk factors are significantly and independently associated with symptomatic lumbar disc herniation. These findings provide further confirmation that atherosclerosis may be involved in spinal disc degeneration. Modification of risk factors, particularly smoking, may also prove to be beneficial.

\section{STROKE}

\section{K-01}

Selection of acute ischemic stroke patients for intra-arterial thrombolysis with prourokinase using ASPECTS

Michael D. Hill (Calgary, Alberta), Howard Rowley (Madison WI, USA), Felix Adler (Boston MA, USA), Michael Eliasziw (Calgary Alberta), Anthony Furlan (Cleveland OH, USA), Randall Higashida (San Francisco CA, USA), Lawrence Wechsler (Pittsburgh PA, USA), Carolyn Firszt (Chicago IL, USA), Alastair M. Buchan (Calgary, Alberta) for the PROACT-II Investigators

Winner of the Canadian Neurological Society's Frances McNaughton Memorial Prize. See page 6.

\section{K-02}

\section{C-reactive protein in ischemic stroke and its etiological subtypes}

Graeme J. Hankey, Joln W. Eikelboom, Ross I. Baker, Andrew McQuillan, Jim Thom, Janelle Straton, Qilong Yi, (Perth, Australia and Toronto, Ontario)

Background: The possible role of C-reactive protein in the etiology and prognosis of ischaemic stroke remains to be clearly defined. The aims of this study were to determine (1) whether Creactive protein levels are elevated in patients with stroke and (2) whether levels may be higher in patients with etiologic subtypes of stroke caused by large or small artery disease ('atherogenic hypothesis') or whether they may be higher in patients with more extensive cerebral infarction caused by large artery or cardiogenic embolism ('inflammatory marker hypothesis').

Methods: We conducted a case-control study of 199 hospital cases with a first-ever ischemic stroke and 202 randomly selected community controls stratified by age, sex and postal code. Using established criteria, cases of stroke were classified by etiologic subtype in a blinded fashion and the prevalence of conventional vascular risk factors and $\mathrm{C}$-reactive protein levels were determined in stroke cases and controls.

Results: Blood levels of C-reactive protein measured within seven days of acute stroke were significantly higher in cases compared with controls $(8.50$ vs. $2.18 \mathrm{mg} / \mathrm{L}, \mathrm{P}<0.0001)$ and remained significantly elevated in stroke survivors at 3-6 months of follow-up ( 3.35 vs. $2.18 \mathrm{mg} / \mathrm{L}, \mathrm{P}=0.003$ ). Compared with the lowest quartile, the upper three quartiles were associated with an adjusted odds ratio (OR) of ischemic stroke of $1.9(95 \% \mathrm{Cl}: 1.0-3.8)$ for the second quartile, 5.8 (95\% CI: 2.9-11.4) for the third quartile, and $16.9(95 \% \mathrm{Cl}: 7.9-36.1)$ for the fourth quartile (P for trend $<0.0001$ ). Comparing the upper with the lower quartile, the strongest association was with etiologic stroke subtypes caused by large artery disease (OR 52.5; 95\% CI: 13.4-205) and embolism from the heart (OR 56.1; 95\% CI: 11.3-278), with a much weaker association with small artery disease (OR 2.4; $95 \%$ Cl: 0.8-6.0).

Conclusion: There is a strong, independent relationship between elevated blood levels of C-reactive protein and ischemic stroke, particularly due to large artery disease and embolism from the heart, with a much weaker association with small artery disease. Our results are consistent with the inflammatory marker hypothesis of Creactive protein as a marker of the burden of atherosclerosis and extent of ischemic cerebral injury.

\section{K-03}

\section{Stroke following neck manipulation}

V. Beletsky (Toronto, Ontario), M. Eliasziw (Calgary, Alberta), A. Shuaib (Edmonton, Alberfa), A. Woolfenden (Vancouver, British Columbia), J.W. Norris (Toronto, Ontario) for the Canadian Stroke Consortium

The cervical arteries are vulnerable to dissection following a variety of trauma, particularly the vertebral artery due to its tortuous course prior to entering the skull. Previous data from the Canadian Stroke Consortium (CSC) as well as other national studies indicates that dissection is probably the commonest cause of stroke in patients $<45 \mathrm{yrs}$. 
The CSC is currently conducting a study of cervical arterial dissection and of 173 cases so far in this prospective survey, trauma severe or trivial was the cause in $109(63 \%)$. In 45 patients $(26 \%)$ the dissection was apparently spontaneous, while in $41(38 \%)$ of the 109 in the traumatic group neck manipulation was the apparent cause. There were 32 vertebral and nine carotid dissections, with a predominance in young women ( 29 women, $40 \pm 11$ yrs.) vs. 12 men $(48 \pm 17 \mathrm{yrs})$. In $16 \%$ of our total cohort there was a variety of congenital abnormalities, such as Marfan's syndrome and fibromuscular dysplasia, which may be a factor in selective vulnerability of the arteries.

Data from Statistics Canada 2000 indicates that the incidence of ischemic stroke from dissection in patients $<45 \mathrm{yrs}$ is about $700 / \mathrm{yr}$, and since our data indicate $20 \%$ associated with the neck manipulation (from entire cohort), we believe that the incidence is about 140 patients/yr, suggesting gross under-reporting of this important cause of stroke, especially in young women.

\section{K-04}

MR perfusion thresholds in stroke thrombolysis are time dependent

K. Butcher, M. Parsons, A. Rawlinson (Melbourne, Australia),

A. Barber (Auckland, NZ), J. Chalk (Brisbane, Australia),

G. Donnan, B. Tress, S. Davis (Melbourne, Australia)

Background: Perfusion weighted MRI has been shown to be useful in the early identification of cerebral tissue at risk of infarction during acute ischemia. Identification of threshold perfusion measures which lead to infarction may assist in the prediction of the response to thrombolysis.

Methods: Mean transit time (MTT) maps were generated in 35 acute stroke patients ( 17 tPA treated, 18 control patients) imaged sub-six hours from symptom onset. Day 90 outcome infarcts (T2 weighted MRI) were superimposed on acute MTT maps. MTT was then calculated for two regions: infarcted and salvaged tissue.

Results: The average delay in MTT, relative to the unaffected side, was 8.2 seconds in infarcted tissue and 6.5 seconds in salvaged tissue $(p<0.001)$. In patients in whom recanalization occurred (13 tPA and five placebo patients), tissue salvage occurred in regions with significantly prolonged MTT compared to patients without recanalization ( 7.5 vs 5.6 seconds, $\mathrm{p}<0.05$ ). In recanalized patients, an inverse correlation $(R=0.93, p<0.00 I)$ was found between time of initial MRI scan and MTT delay in salvaged tissue.

Conclusions: Recanalization, which is enhanced by tPA, allows salvage of more severely hypoperfused tissue. When considering thrombolysis, perfusion thresholds for infarction need to be assessed in the context of symptom duration.

\section{K-05}

\section{Auditing carotid endarterectomy: A regional experience}

\section{J.M. Findlay, L. Nykolyn, T. Lubkey, M. Mouradian,}

\section{A. Senthilselvan, (Edmonton, Alberta); J. Wong, (Calgary, Alberta)}

Background: Proof from RCTs that carotid endarterectomy (CEA) is effective in stroke prevention has resulted in a large resurgence in its use in recent years. We wished to determine if patients in our region were being selected and treated with complication rates consistent with the randomized trials
Methods: We have conducted four audits of CEAs performed in our region since 1994, each followed by feed-back of results and information to the participating surgeons. Operations for $70 \%-99 \%$ symptomatic stenosis were considered appropriate, those for $50 \%$ $69 \%$ symptomatic and $60 \%-99 \%$ asymptomatic stenosis were considered uncertain, and all others including those in medically or neurologically unstable patients were designated inappropriate. In part 4 the referral source and nature of the patients was also determined.

Results: The first audit (part 1) found that $33 \%$ of 291 CEAs were appropriate, $48 \%$ were uncertain, $18 \%$ were inappropriate, and $40 \%$ of patients were asymptomatic. Significant, successive improvements in CEA performance have been noted in subsequent audits. In part 4 appropriate indications increased to $58 \%$ of 222 CEAs, there were no inappropriate procedures, and the overall stroke and death rate was $2.3 \%$ compared to $5.2 \%$ in part 1 . The number of uncertain candidates, consisting mainly of asymptomatic patients, has not changed significantly. In part 4 it was found that the majority of patients $(69 \%)$ were referred for surgery directly from GPs, including $58(73 \%)$ of the 80 asymptomatic patients who underwent CEA.

Interpretation: Regular auditing and feedback of results and information to surgeons has resulted in significant and continued improvements in the surgical performance of CEA in our region. Since the majority of patients are referred directly by family physicians it is important that this group of doctors be familiar with current CEA guidelines.

\section{K-06}

\section{Alterations in lipid profile after stroke study}

\section{B. Yan, M. Parsons, S. McKay, B. Infeld, D. Campbell, R. Caajko,} S. Davis (Melbourne, Australia)

Background: Although acute decrease in total cholesterol (TC) is well-documented in myocardial infarction, stroke studies have produced conflicting results. We aimed to determine the optimal time for lipid measurements after stroke. We hypothesized that TC would acutely decrease post stroke and return to baseline by three months.

Methods: A prospective, observational study of 50 patients (age $73 \pm 12.4$ years) who presented with acute ischaemic stroke. Of these, 13 were already on statins and $I 2$ were commenced on statins. Currently, 24 have completed three month follow-up, five died, five were lost to follow-up. Fasting lipid profile (TC, LDL, HDL, TG) was measured $<48$ hours, four weeks and 12 weeks.

Results: In patients who were statin naive $(n=9)$, there was an increase in TC of $1.0 \pm 0.91$ at three months ( $p=0.007)$. In patients who continued on statins $(n=7)$, there was a trend to increased TC of $0.54 \pm 0.90(p=0.12)$. In patients who were commenced on statins $(n=8)$, there was a decrease in TC of J.85 $\pm 0.99(p=0.001)$.

Conclusions: Cholesterol levels in acute stroke are an unreliable measure of lipid status. Initiation of statins should be based on measurements taken three months after stroke. 


\section{K-07}

\section{Neuroprotection by a novel drug delivery system for acute} cerebral ischemia

Y. Takanashi, I. Yamamoto, (Yokohama, Japan); T. Ishida, MJ. Kirchmeier, A. Shuaib, T.M. Allen (Edmonton, Alberta)

Background: To date, pharmacological approach to cerebral ischemia is unable to attain effectively high concentrations of the drugs in the cerebrospinal fluid (CSF) without precipitating systemic adverse effect. The purpose of this study is to develop a liposomal drug delivery system in a sustained-release form of protein kinase inhibitors fasudil in the CSF, resulting in neuroprotection against cerebral ischemia.

Methods: Focal cerebral ischemia was induced in 40 rats by middle cerebral artery occlusion using an intraluminal suture technique. Each rat randomly received either $0.25 \mathrm{mg}$ liposomeentrapped fasudil or drug-free liposomes via the cisterna magna two hours after ischemic insult. Neurological condition, the infarct size, and the concentration of liposome-entrapped fasudil in the CSF were assessed at 24 and 72 hours after ischemia.

Results: In the treated animals, neurological outcomes as well as neuroprotection against cerebral ischemia were significantly ameliorated $(\mathrm{P}<0.00 \mathrm{I})$. However, there was no statistical difference between 24 and 72 hours. At 24 hours post-administration, $80 \%$ of fasudil was released from liposomes in the CSF.

Conclusions: A single intrathecal injection of liposomal fasudil is effective in preventing acute ischemia in a rat model. This new approach for acute cerebral ischemia may have significant potential for use in the clinical setting.

\section{K-08}

Automated detection and volume measurement of ischaemic stroke on DWI and T2 images

T.G.Phan, P. Wright, I. Indira, G.A. Donnan, D. Reutens (West

Heidelberg, Australia)

Aim: To develop a reliable automated method for detection and volume measurement of ischaemic stroke on diffusion-weighted images (DWI) and T2 weighted images.

Background: Automated detection and volume measurement of acute ischaemic stroke has assumed great clinical and research significance with the drives towards treatment of ischaemic stroke guided by diffusion and perfusion MR studies. Manual measurement of infarct volume on DWI and T2 images is difficult and time consuming. This is particularly so for DWI images as the apparent diffusion coefficient (ADC) values are provided on separate map. Ischaemic tissue is defined by the presence of high signal intensity on DWI and low values on ADC map. Thus not taking the ADC values into account may lead to errors. On T2 images, ischaemic tissue is defined by the presence of high signal intensity. Map of cerebrospinal fluid was used to remove this source of bright signal. An automated algorithm allows consistent ischaemic tissue volume measurement and without the bias introduced by the different observers.

Method: We wrote a simple algorithm in Matlab language on an IBM compatible personal computer. This algorithm uses a combination of cluster analysis, histogram signal intensity thresholding and for segmentation of DWI images we add thresholding using the mean ADC value $\left[800 \times 10^{-6} \mathrm{~mm}^{2} / \mathrm{sec}\right)$. This algorithm successfully processed moderate to large size ischaemic stroke in the distribution of the middle and posterior cerebral artery territory.

Conclusion: Our algorithm for detection and volume measurement of ischaemic stroke on DWI and T2 images is reliable, fully automated and requires no user interaction.

\section{VASCULAR NEUROSURGERY}

\section{L-01}

Nine year experience in endovascular treatment of unruptured cerebral aneurysms at a single institution

D. Roy, A. Weill, F. Guilbert, SA. Georganos, J. Raymond (Montreal, Quebec)

Background: Endovascular treatment of unruptured aneurysms, including evolution of results and indications, are reviewed.

Methods: All patients treated by coil embolization of an unruptured cerebral aneurysm between 1992 and 2001 at the Centre Hospitalier de l'Université de Montréal were included. Prospective observation included clinical presentation, treatment complications, as well as early and late clinical and morphological results.

Results: Among a total of 469 aneurysms treated with selective coil embolization, 196 in 181 patients were unruptured. Ophthalmic segment aneurysms represent $40 \%$. There was no mortality. A permanent complication occurred in $3.9 \%$ of patients (GOS I or II). All treated patients had a good outcome. No patient bled during a mean follow-up of 28 months. Morphological results showed $90.8 \%$ complete occlusion/residual neck, $4.6 \%$ residual aneurysm and $4.6 \%$ failure. Recurrence rate was $6 \%$.

Conclusion: In unruptured aneurysms, the low morbidity of endovascular treatment favors it as a first-line approach in selected cases. Aneurysmal recurrences, a shortcoming regarding long-term protection, is undergoing further investigation.

\section{L-02}

Safety of surgical treatment for unruptured middle cerebral artery aneurysms

\section{N.Heran. G. Redekop (Vancouver, British Columbia)}

Background: The management of unruptured intracranial aneurysms is controversial. Endovascular approaches are increasingly employed as an alternative to open microvascular surgery. Middle cerebral artery aneurysms are infrequently suitable for endovascular treatment and represent a subgroup of aneurysms for which open surgery is the primary therapeutic modality. The experience of a single cerebrovascular surgeon in the management of unruptured, non-giant middle cerebral artery aneurysms (MCA) was reviewed in order to determine the procedural risks and outcomes.

Methods: A consecutive series of 33 patients who underwent 38 craniotomies for clip ligation of unruptured MCA aneurysms was reviewed. Information was primarily obtained from the surgeon's prospective database and supplemented by chart and imaging review as well as telephone follow-up. 
Results: There were 24 females and nine males, ranging in age from 31 to 73 years (mean $=49$ years). Five patients with bilateral unruptured MCA aneurysms underwent bilateral craniotomies. Eight patients had concurrent clipping of another unruptured ipsilateral anterior or posterior circulation aneurysm at the time of surgery. Thirteen patients had a history of subarachnoid hemorrhage and had previously undergone contralateral craniotomy $(n=11)$ or endovascular therapy $(n=2)$ to repair a ruptured aneurysm in a different location. One patient experienced a new, permanent neurological deficit $(2.6 \%)$. Three patients $(7.8 \%)$ experienced transient morbidity (wound infection in one, urinary retention in one, and postoperative seizure in one). Two patients experienced minor cranial nerve morbidity related to concurrent clipping of other aneurysms.

Conclusions: Unruptured MCA aneurysms can be repaired surgically with very low rates of permanent morbidity $(2.6 \%)$. Although 16 patients (48\%) underwent bilateral craniotomies, there did not appear to be an increased risk of complications in this subgroup.

\section{L-03}

Endovascular and surgical management of multiple intradural aneurysms: Review of 122 patients managed between 1993 and 1999

P. Porter (Toronto, Ontario), M. Mazighi, G. Rodesch. H. Alvare:N. Aghakhani, P. Lasjaunias (Paris, France)

Background: This study was undertaken to review the unique challenges in the management of patients with multiple intradural aneurysms treated at a single institution.

Methods: Consecutive patients with multiple intradural aneurysms managed from 1993-1999 were studied. Data was prospectively collected on all aneurysm patients in a computerized database. The multiple aneurysm subgroup was retrospectively analyzed.

Results: The 122 patients had a total of 305 aneurysms. In most patients presenting with subarachnoid hemorrhage $(\mathrm{SAH})$, the aneurysm responsible for the bleed could be identified with reasonable certainty, as confirmed by subsequent surgical and autopsy findings. Irregularity of the aneurysm (false sac or polylobulation) was the most useful criterion. Failure to recognize all aneurysms on the original angiogram remained an uncommon but clinically important problem. Posterior inferior cerebellar and anterior communicating artery aneurysms were disproportionately more likely, and para-ophthalmic less likely, to be responsible for the SAH. Patients with uncertainty regarding the site of bleeding tended to have all aneurysms treated, and in a shorter time. Surgical and endovascular complication rates and patient outcomes were not dissimilar from those expected for single aneurysm patients. During follow-up the hemorrhage rate from unruptured aneurysms was $1.1 \%$ per patient-year. De novo aneurysm formation occurred in $0.76 \%$ of patients per year.

Conc/usions: Although patients with multiple intradural aneurysms have more complex management issues than those with single aneurysms, good outcomes can be achieved with appropriate use of endovascular and/or surgical therapy. The goal in the acute setting following SAH is recognition of all aneurysms and urgent treatment of the one responsible for the hemorrhage. When there is uncertainty, more than one aneurysm may need to be treated. Decisions on subsequent treatment of remaining unruptured aneurysms must be individualized.

\section{L-04}

Direct surgical clipping of large and giant internal carotid artery (ICA) aneurysms

\section{Shedid, J.-L. Caron, M.W. Bojanowski (Montreal, Quebec)}

Background: Large and giant ICA aneurysms are difficult surgical lesions and intravascular techniques are associated with a high incidence of recurrence. We reviewed our recent experience with direct surgical approach of these challenging ICA aneurysms.

Methods: We retrospectively studied a series of 22 consecutive patients treated by direct surgical approach for large and giant ICA aneurysms between 1995 and 2001 . The clinical presentation, the radiological findings and the outcome were analyzed along with the surgical technical aspects.

Results: The majority of patients were admitted following a subarachnoid hemorrhage. There were four aneurysms of the ophthalmic segment of the ICA, 13 of the supraclinoidal segment and five were located at the bifurcation of the ICA. All ophthalmic segment aneurysms were operated by the combined extra-intradural approach. Endovascular coiling ivas used in one patient for residual neck. The outcome was favourable for these patients. For the supraclinoidal segment one patient had a transient hemiparesis and hemianopia. Two deaths were $\mathrm{H} \& \mathrm{H}$ grade 5 on admission. All others patients had a favourable outcome including the five patients with ICA bifurcation aneurysms.

Conclusion: With contemporary strategies and techniques. direct surgical therapy is often the best method of securing a large or giant aneurysm of the ICA. The selective criteria will be discussed.

\section{L-05}

A cost effectiveness analysis of surgical clipping versus guglielmi detachable coil (GDC) obliteration of asymptomatic cerebral aneurysms

\section{S.D. Christie, I. Mende:. T. Kennedy, R.O. Holness, R. Vandorpe,} W. Maloney, I. Fleetwood (Halifar, Nova Scotia)

Background: Recently there has been an increased interest in endovascular techniques to obliterate cerebral aneurysms. The literature suggests that complication rates from GDC coiling are equal or superior to those of conventional craniotomy. However, to date there has been no clinical data published comparing the cost effectiveness of these two techniques. Our objective was to determine the relative cost of treating asymptomatic cerebral aneurysms by either GDC obliteration or conventional surgical clipping.

Methods: All patients undergoing treatment for asymptomatic aneurysms at our centre between 1999 and 2001, inclusive, were identified. A detailed analysis was performed to calculate the cost per patient for the purposes of comparing the two groups.

Results: 32 patients underwent coiling compared to 28 who underwent surgical clipping. Coiled patients had a shorter hospital stay compared to the surgical group $(4.06 \pm 1.8$ days versus $9.11 \pm$ 4.7 days, respectively; $p<0.000007$ ). The overall mean cost for both groups, including the costs of hospital stay, procedures and 
materials, was $\$ 8478.26 \pm 2226.61$ for coiling patients versus $\$ 8626.81 \pm 2350.90$ for clipping patients $(p=0.8)$.

Conclusion: Although patients undergoing coiling have a significantly shorter hospital stay, there was no difference in average cost between the two groups.

\section{L-06}

Vessel reconstruction in patients with cerebral aneurysms: a contemporary experience

\section{G. Luzardo-Small, G.T. Mandybur, G.S. Dhillon, I.B. Ross (Jackson, USA)}

Background: Our group has a primarily endovascular thrust, reserving surgery for patients whose cerebral aneurysms have endovascularly unfavourable anatomy or have failed coiling. The purpose of this study was to analyze our prospectively gathered data to determine anatomical results.

Methods: All patients were treated between March, 2000 and November, 2001. Cases were deemed to have succeeded or failed based on whether or not the intervention, be it surgical or endovascular, achieved the treatment goal of arterial reconstruction/repair.

Results: 51 endovascular and 23 surgical interventions were performed. No middle cerebral artery aneurysms were coiled and only one posterior circulation aneurysm was surgically treated. Reasons for the decision to surgically explore included: unfavourable anatomy (aneurysm too small or with too wide a neck), presence of significant cerebral vasospasm, intracerebral clot requiring evacuation, presence of multiple adjacent aneurysms. Only two aneurysms failed endovascular attempts (4\%) and went to surgery. Three of the surgical cases did not achieve anatomic success (13\%). The clinical outcomes were good in two of these three cases; none were candidates for further endovascular intervention.

Conclusions: The majority of cerebral aneurysms can be treated endovascularly. There is a high success rate for patients undergoing these treatments.

\section{L-07}

Quantitative cerebral perfusion using bolus-tracking CT: preliminary experience with revascularization procedures

M.J.Hogan, M. Goyal, P. Kalapos, V.F. DaSilva, A. Douen, C. Freeman (Ottawa, Ontario)

Background: It is now possible using standard $\mathrm{x}$-ray computerized tomography (CT) to easily obtain quantitative measurements of regional cerebral blood volume (CBV), cerebral blood flow (CBF) and mean transit time (MTT) in patients with cerebrovascular disease. However, the potential impact of this new knowledge on patient management is unknown. We report our initial experience with CT perfusion measurements in six patients undergoing revascularization for chronic symptomatic cerebrovascular disease.

Methods: Perfusion measurements were performed before and after a revascularization procedure (three carotid endarterectomies, two carotid angioplasty and stenting procedures and one basilar angioplasty) using bolus-tracking CT on a GE Medical Systems HiSpeed scanner. Omnipaque $300(0.5 \mathrm{ml} / \mathrm{kg})$ was administered intravenously at a rate of $4 \mathrm{ml} / \mathrm{sec}$ and tracked by serial CT scans at one second intervals over 45 seconds at a selected axial level. CBV, CBF and MTT were calculated using a GE Advantage Workstation.

Results: Three patients demonstrated regional cerebral hypoperfusion defined by both decreased CBF and increased MTT in non-infarcted brain on the initial study. Improved perfusion was observed in vascular territories directly associated with the revascularization procedure in these patients. In the remaining patients initial perfusion deficits and subsequent changes after revascularization were less marked.

Conclusions: Bolus tracking CT can identify patients with chronic cerebral hypoperfusion and can define changes following revascularization. This technique may have a role in the investigation and monitoring of therapy in patients with cerebrovascular disease.

\section{L-08}

The anterolateral skull base approache improves outcome over the pterional for the repair of ruptured anterior communicating artery aneurysms

\section{C.A.R.Bruce (UWI Jamaica); M.D. Cusimano, F. Afzal (Toronto, Ontario)}

Background: The orbitocranial approach (OC) is ideal for surgery of lesions affecting the skull base, however, the pterional (P) approach remains the standard approach used for treatment of anterior communicating artery aneurysms (ACA). This study assesses the outcome of patients with ruptured ACA operated via both approaches.

Methods: A total of 108 patients had subarachnoid hemorrhage (SAH) due to a ruptured ACA. 30 patients, median age of 63 years, were operated on via the $\mathrm{OC}$ approach. The median Hunt-Hess was 3.78 patients, median age of 55 years had a pterional approach, median Hunt-Hess 2.

Results: Intraoperative rupture occurred in $30 \%$ of patients in the OC group and in $45 \%$ in the pterional group. Gyrus rectus resection was $6.7 \%$ in $\mathrm{OC}$ approach and $35.9 \%$ in the pterional group. Postop CT infarction was seen in $27 \%$ of OC patients and $31 \%$ of pterional patients. More cognitive deficits were seen in the pterional patients $59.3 \%$ and $23.3 \%$ in the OC patients. Median Glasgow Outcome Score was 4 in both groups and mortality $13 \%$ in both groups.

Conclusion: The orbitocranial approach to ruptured ACA aneurysms is associated with excellent outcome equivalent to or better than the traditional pterional approach. 


\section{Poster Presentations}

\section{STROKE}

\section{P-001}

\section{Neurological complications of cardiac transplant: a university hospital experience}

\section{N. Amir, A. Shuaib, D. Modry, A. Koshal, (Edmonton, Alberta)}

Background: Since the first heart transplant in 1967, many authors have reported the complications of heart transplant on CNS which could range from encephalopathy to fatal ischemic or hemorrhagic stroke.

Methods: Between 1985 and 2001, 335 orthotopic cardiac transplantations were performed on 334 patients for a variety of cardiac diseases leading to end stage refractory cardiac failure. A retrospective chart review was done to identify those patients who developed any neurological complication.

Results: Forty patients (11.9\%) developed neurological complications. Twenty-four patients $(7.1 \%)$ suffered from encephalopathy, eight $(2.3 \%)$ had single or multiple seizures perioperative or postoperatively, seven $(2.0 \%)$ suffered from ischemic stroke and three $(0.8 \%)$ had intra-cerebral hemorrhages. There were three deaths. One patient died due to severe peri-operative hypotension and subsequent renal failure, second death was due to hypoplastic left heart syndrome and third death was due to pneumonia and respiratory failure, the patient also suffered from a left MCA stroke.

Conclusions: We concluded that neurological complications are infrequent after cardiac transplantation. Embolic phenomena are probably more frequent as a cause of stroke in patients who suffer from peri- or postoperative cerebrovascular insult as compared to atherosclerotic disease or global hypoperfusion syndromes.

\section{P-002}

The presence of a middle cerebral artery occlusion increases with quantified CT ischemic change: therapeutic targets for future acute stroke studies

\section{P.A.Barber, A.M. Demchuk, M.D. Hill, J.H.W. Pexman,}

\section{M.E. Hudon, R. Frayne, Alastair M. Buchan (Calgary, Alberta)}

Introduction: Non-contrast $\mathrm{CT}$ is the imaging modality most accessible to clinicians treating acute stroke and has been shown to reliably predict functional outcome before thrombolytic therapy. What is not known is the probability of an acute vascular occlusion of the middle cerebral artery or its branches according to the extent of CT ischemia in acute stroke.

Methods: Patients presenting with disabling ischemic stroke had a CT scan $(<6 \mathrm{~h})$ prior to MRI ( $<7 \mathrm{~h})$. A quantitative scoring system (ASPECTS) was applied to CT and DWI at baseline and follow-up ( $<36 \mathrm{~h}$ ) by five independent observers with only knowledge of the stroke side. The presence of the HMCAS and the MCA dot sign was also evaluated. Each observer, after an interval period assessed the 3D TOF MRA in the anterior circulation for areas of decreased signal in the MCA territory, with absent signal taken to represent severely reduced or absent flow. Consensus agreement was determined by using three out of five of the independent observations.

Results: A total of 107 patients considered to have anterior circulation strokes had baseline CT and MRI. The median CT ASPECTS was 9. There was a total of 11 HMCA signs and 19 "dot" signs, of which four had HMCA and "dot" signs. The MRA confirmed absence of flow in both $\mathrm{M} 1$ and $\mathrm{M} 2$ in 29 cases and the $\mathrm{M} 2$ alone in 16. HMCAS had sensitivity of 0.42 [95\% CI $0.23-0.63$ ], specificity $1.0[0.93-1.0]$ for signal intensity in the $\mathrm{M} 1$ portion of the MCA. The MCA "dot" sign had sensitivity of $0.52[0.31-0.73]$ and specificity 0.95 [0.85-0.99] for signal intensity in the M2 portion of the MCA. The probability of a MRA flow signal abnormality increased inversely with decreasing ASPECTS (Spearman correlation $-0.63, P<0.001)$. ASPECTS values of 10,9 and 8,7 and 6,5 and below were associated with the following MRA signal abnormalities: $12,67,81$, and $91 \%$ respectively.

Conclusions: This study demonstrates that the extent of CT ischemic change quantified by ASPECTS increases the probability of a MCA occlusion and this finding, together with the identification of a hyperdense CT arterial sign should form the basis for selecting patients for future therapeutic clinical trials.

\section{P-003}

\section{Optimal neuroimaging in cervical arterial dissection}

V. Beletsky (Toronto, Ontario); F. Morriello (Bologna, Italy); J.W. Norris (Toronto, Ontario) for the Canadian Stroke Consortium

Cervical arterial dissection (CAD) is a frequent cause of ischemic stroke in the young. Catheter angiography (IA-DSA) is the accepted standard for confirming the clinical diagnosis, but carries $1 \%$ risk of stroke and may not always be accurate. The Canadian Stroke Consortium is conducting a prospective study of cervical arterial dissection, with a view to determining the best treatment regimen. This depends upon the confirmation of the clinical diagnosis by neuroimaging.

In 156 patients with $\mathrm{CAD}, 72 \%$ (112 patients) underwent IADSA, in which dissection was diagnosed in $84 \%$ (93) of cases. $42 \%$ (66) of patients underwent MRI studies and in $21 \%$ (33) MRA, confirming dissection in $37 \%$ and $79 \%$. Combination MRI/MRA diagnosed dissection in $86 \%$ of cases (18 of 21 ), almost the same figure as IA-DSA. Duplex scanning was performed in only $19 \%$ (31) cases and was non-specifically abnormal in $52 \%$ ( 16 of these).

Since the MRI/MRA combination is as accurate as IA-DSA and is also non-invasive, we believe this is the method of choice. Duplex scanning is clearly not a favored technology because of limitations of the technology and its non-specific nature.

\section{P-004}

\section{Obstructive sleep apnoea and stroke}

S. Broadley, L. Jorgensen, A. Cheek, S. Salonikis, J. Taylor, P. Thompson, R. Antic (Adelaide, Australia)

Background: Obstructive sleep apnoea (OSA) is an independent risk factor for hypertension, which is a major risk factor for stroke. 
We have therefore studied the incidence and associations of OSA in a cohort of stroke patients.

Methods: Consecutive patients admitted with acute stroke $(n=81)$ were considered for study. In addition to a standard neurological assessment, all patients were assessed using the Parramatta Hospitals dysphagia score, modified Barthel index and a multivariate apnoea (MAP) index (Maislin et al, 1995) to assess prior symptoms of OSA. Sleep studies were performed within the first few days of admission using a portable diagnostic system, Embletta ${ }^{\circledR}$ PDS.

Results: Of 53 patients studied, $27(51 \%)$ had evidence of sleepdisordered breathing. In 21 (40\%) this was due to OSA ( 10 of whom also had periodic breathing) and a further six patients had periodic breathing alone. The apnoea-hypopnoea index was significantly associated with the MAP index of prior OSA symptoms, but not with history of hypertension, dysphagia score, type or severity of stroke.

Conclusions: OSA is common after acute stroke and exceeds rates seen in the general population ( $40 \%$ vs. $7 \%$ ). The association of OSA with the MAP index suggests that OSA is frequently preexistent.

\section{P-005}

\section{Echoplanar imaging thrombolysis evaluation trial: EPITHET}

The EPITHET Investigators: Ken Butcher, Stephen Davis, Geoffrey Donnan, Mark Parsons, Peter Wright (Melbourne, Australia), Alan Barber (Auckland, NZ), Richard Gerraty, Judith Frayne, Paul Talman, Chris Bladin (Melbourne, Australia), Chris Levi (Newcastle, Australia), Geoffrey Herkes, John Watson (Sydney, Australia), Graeme Hankey (Perth, Australia), Jonathan Chalk (Brisbane, Australia), David Schultz (Adelaide, Australia)

Background: EPITHET is a multi-centre trial, aimed at identification of echoplanar imaging (EPI) criteria for thrombolytic therapy in acute stroke. Mismatch between perfusion weighted image (PWI) lesions and a diffusion weighted image (DWI) lesions putatively represents the penumbra. We hypothesize that patients with PWI-DWI mismatch will benefit from tPA 3-6 hours after symptom onset.

Methods: This is a double blind, randomised-controlled trial of tPA versus placebo in 100 eligible patients with hemispheric infarction 3-6 hours after stroke onset. Patients receive either tPA (NINDS protocol) or placebo (1:1). Neurological impairment and functional scores are measured by a neurologist, blinded to the EPI results and treatment designation. Enrolled patients have standardized DWI, PWI and magnetic resonance angiography (MRA) sequences performed prior to and 3-5 days post-treatment. Final infarct volume (T2-WI) is measured at 90 days.

Results to date: Active enrolment of patients began in September. Enrolment will continue until early 2003.

Conclusions: This is the only randomised controlled trial of the effectiveness of t-PA used in conjunction with EPI. Given the lack of efficacy of tPA beyond three hours in previous stroke trials, this would appear to be the most promising route for extension of the current treatment time window.

\section{P-006}

\section{Hyperglycemia augments stroke expansion on MRI}

S.M. Davis, T.A. Baird, M.W. Parsons, P.A. Barber, P.G. Colman, P.M. Desmond, B.M. Tress, G. Jerums, B. Chambers (Melbourne, Australia)

Background: Hyperglycemia in acute stroke is associated with increased mortality and morbidity. Animal studies suggest infarct expansion may be responsible.

Aims: To correlate serial blood glucose levels following ischaemic stroke with infarct expansion using diffusion weighted MRI (DWI).

Methods: Recruitment of patients less than 24 hours after anterior circulation ischemic stroke. Continuous glucose monitoring for 72 hours using a subcutaneous glucose sensor (MiniMed CGMS). DWI performed before sensor insertion and following removal.

Results: In 19 patients a mean of 584 sensor readings per patient were obtained. Sensor readings correlated with concurrent 6-hourly fingerprick glucose $(r=0.92, p<0.005)$, but were lower $(6.7$ v. 7.3 $\mathrm{mmol} / \mathrm{l}, \mathrm{p}<0.05)$. Subjects with infarct expansion (10/19) had higher mean sensor glucose ( 7.1 v. $5.3 \mathrm{mmol} / \mathrm{l}, \mathrm{p}=0.04)$. Mean sensor glucose and number of sensor readings $>7 \mathrm{mmol} / \mathrm{l}$ both correlated with volume of infarct expansion $(r=0.52,0.62 \mathrm{p}<0.05)$. Mean fingerprick glucose did not significantly correlate with volume of expansion. Neither presence of diabetes or NIHSS influenced volume of expansion.

Conclusions: Hyperglycemia following ischemic stroke predicts infarct growth. Continuous glucose monitoring provides increased sensitivity and allows more accurate prediction of stroke evolution. Trials of intensive insulin therapy are warranted in acute stroke

\section{P-007}

\section{Aortic arch related cerebral hazard (ARCH)}

G. Donnan, S. Davis, B. Chambers (Victoria, Australia); G. Hankey (Western Australia, Australia); E. Jones (Victoria, Australia); C. Levi (New South Wales, Australia); S. Read (Queensland, Australia); J. Frayne (Victoria, Australia)

Background: Aortic arch atheroma has emerged as a significant risk factor for ischaemic stroke (relative risk 3-4). Currently, there is no evidence as to what form of therapy is likely to minimise the chance of recurrent stroke. For aortic arch atheroma of thickness $\geq 4 \mathrm{~mm}$ or mobile, the risk of stroke or death per year is about $14 \%$. Given this high stroke or death risk, the study will test whether the most logical forms of therapy would be either anti-coagulation (warfarin) or combination antiplatelet therapy (aspirin plus clopidogrel).

Methods: Sample size: 1500 patients for a power of $90 \%$, and alpha.05. Therapy: Warfarin INR $2.0-3.0$ vs aspirin $75 \mathrm{mg}$ to $325 \mathrm{mg}$ daily plus clopidogrel $75 \mathrm{mg}$ daily. Study Design: Open randomised controlled trial. The primary outcome events are a blinded composite of recurrent stroke, AMI, peripheral embolism or vascular death. The study duration will be five years within which there will be a three year recruitment period.

Results: Recruitment is commencing across 15 sites within Australia and centres in Europe and North America are planned.

Conclusions: This is the first trial of therapy for this important ischaemic stroke sub-set. 
"Malignant" carotid artery dissection

J.M. Findlay, R. Ashforth, N. Deane, (Edmonton, Alberta)

Purpose: Carotid artery dissection resulting in occlusion or severe narrowing and massive intracranial embolism can result in life-threatening hemispheric ischemia. Aggressive measures may be necessary to salvage life and minimize morbidity in this situation.

Patients and Methods: We have treated two women who presented within one hour of spontaneous cervical ICA dissection causing hemiplegia, forced head and eye deviation, and declining consciousness. The first had an occlusion through which a catheter could not be passed, so intracranial thrombolysis was achieved with a microcatheter navigated through the posterior circulation. Surgical intimectomy and thrombectomy of the dissected ICA was carried out using a Fogarty balloon catheter passed up the ICA, followed by endovascular stenting of the reopened ICA. The second patient underwent intracranial embolectomy, and after an unsuccessful attempt of stenting the dissected ICA, surgical reopening using a Fogarty catheter. Both patients suffered basal ganglionic infarcts, but most of the middle cerebral artery territories were preserved and the patients made satisfactory recoveries.

Conclusions: "Malignant" carotid artery dissection causing occlusion or near occlusion with intracranial embolism is an important cause of severe hemispheric ischemia, and treatment should include aggressive endovascular and sometimes microsurgical interventions when the hemisphere is at risk.

\section{P-009}

Leukoencephalopathy of combined cerebral amyloid angiopathy and cerebral vasculitis: radiological characteristics and response to treatment in eight patients

\section{R.P. Gerraty, S.M. Davis, N. Trost, P.J. Mitchell, P.A. McKelvie,} M.F. Gonzales, D. Freilich, C.A. McLean (Melbourne, Australia)

Background: Cerebral amyloid angiopathy (CAA) is associated with primary angiits of the central nervous system (PACNS) more often than is likely by chance. A characteristic leukoencephalopathy is encountered in many cases, sometimes initially suspicious for cerebral tumour.

Method: Eight patients presenting with hemisphere syndromes had a characteristic leukoencephalopathy on MRI, asymmetrical, with mild mass effect and no enhancement. We performed open cerebral biopsy in each case. Congo red and beta A4 stains were performed on all specimens.

Results: Biopsy confirmed CAA and PACNS in all cases. There were no complications of cerebral biopsy. All patients were treated with corticosteroids and two patients also received cyclophosphamide. All patients improved on treatment. One patient died of pneumonia, a complication of immunosuppression. Followup has been up to five years without relapse.

Conclusions: CAA and PACNS is probably the commonest cause of unexpected leukoencephalopathy in a patient presenting with focal neurology. The diagnosis should be suspected from the MRI. Open cerebral biopsy should be performed to confirm the diagnosis. There is a good response to treatment with corticosteroids and a full recovery can be expected in the majority of cases.

\section{Diffusion and perfusion MRI in subcortical stroke}

R.P. Gerraty, M.W. Parsons (Melbourne, Australia); P.A. Barber (Auckland, NZ); D.G. Darby, P.M. Desmond, B.M. Tress. S.M. Davis (Melbourne, Australia)

Background: The clinical diagnosis of subcortical cerebral infarction is inaccurate for lesion location and pathogenesis. Clinically suspected small perforating artery occlusions may be embolic infarcts, with important implications for investigation and treatment.

Methods: In a prospective series of 106 patients evaluated with acute diffusion (DWI) and perfusion weighted MRI (PWI) within 24 hours of stroke, we enrolled 19 who presented with a lacunar syndrome. Based on the topography, DWI and PWI findings and outcome T2 MRI, we determined whether the mechanism of infarction was single perforating vessel occlusion or large artery embolism.

Results: Thirteen patients had pure motor stroke, two ataxic hemiparesis and four sensorimotor stroke. Six patients had lacunes on DWI, none with a PWI lesion. Four patients had small subcortical and distal cortical infarcts on DWI. Nine had solitary restricted striatocapsular infarcts. Seven of these had PWI studies, five with a PWI lesion. A PWI lesion reliably differentiated striatocapsular from lacunar infarction for solitary small subcortical infarcts $(p=0.03)$.

Conclusion: DWI and PWI altered the final diagnosis of infarct pathogenesis from small perforating artery occlusion to large artery embolism in 13 of 19 patients with clinically diagnosed subcortical infarction. Lacunes cannot be reliably diagnosed on clinical grounds.

\section{P-011}

The VITATOPS (VITAmins TO Prevent Stroke) trial. Rationale and design of an international, large, simple, randomised trial of homocysteine-lowering multivitamin therapy in patients with recent TIA or stroke

Graeme J. Hankey for the VITATOPS trial collaborative group (Perth, Australia)

Background: Epidemiological studies suggest that raised plasma concentrations of total homocysteine (tHcy) may be a common, causal and treatable risk factor for atherothromboembolic ischaemic stroke. Although tHcy can be lowered effectively with small doses of folic acid, vitamin B12 and vitamin B6, it is not known whether lowering thcy, by means of multivitamin therapy, can prevent stroke and other major atherothromboembolic vascular events.

Aim: To determine whether lowering plasma homocysteine concentrations, by means of multivitamin supplements (folic acid $2 \mathrm{mg}$, B6 $25 \mathrm{mg}$, B $12500 \mathrm{mcg}$ ), reduces the risk of stroke, and other serious vascular events, in patients with recent stroke or TIA.

Design: An international, multi-centre, randomised, doubleblind, placebo-controlled trial.

Subjects: Patients with stroke or TIA in the previous seven months.

Primary outcome measure: Non-fatal stroke, non-fatal myocardial infarction, or death due to vascular causes.

Secondary outcome measures: Dementia, depression, TIA, leg amputation. 
Sample size calculation: To reliably identify a $15 \%$ reduction in relative risk of the primary outcome event from $8 \%$ to $6.8 \%$ per year with an alpha of 0.05 and power of $80 \%, 8,000$ patients need to be randomised and followed-up for an average of two years.

Current progress: As of January 1, 2002, a total of 1,550 patients have been randomised in 41 medical centres from 13 countries in four continents: Australia, Austria, Brazil, Hong Kong, Italy, New Zealand, Philippines, Republic of Georgia, Singapore, Sri Lanka, United Kingdom and United States of America.

Conclusion: VITATOPS aims to recruit and follow up 8,000 patients between 2000 and 2004 , and provide a reliable estimate of the safety and effectiveness of dietary supplementation with folic acid, vitamin B12, and vitamin B6 in reducing recurrent serious vascular events among a wide range of patients with TIA and stroke. New investigators who would like to collaborate in the VITATOPS trial are encouraged to visit the VITATOPS website (www.health.wa.gov.au/VITATOPS/).

\section{P-012}

\section{Immunosuppression-responsive reversible lenticulostriate vasculopathy with multiple aneurysms}

\section{N. Heran, M. Heran, J. Lapointe, R.G. Nugent, A.R. Woolfenden (Vancouver, British Columbia)}

Background: Disease affecting only the lenticulostriate vessels is atypically from vasculitic processes. Fusiform aneurysms are rarely seen in primary CNS vasculitis. A patient with progressive irregularity and multiple fusiform aneurysms affecting only the lenticulostriate circulation is presented. These changes reversed with immunosuppressive therapy.

Methods: Case report and review of literature.

Results: A previously healthy 43-year-old female presented with acute hemiparesis. Stroke risk factors were negative. CT head demonstrated an acute internal capsule lacunar infarction. Angiography was not helpful. CSF, rheumatologic and infectious work-up were negative. Fifteen months later, a right basal ganglia hemorrhage occurred and repeat CT head also demonstrated additional bilateral basal ganglia lacunes. Repeat angiography demonstrated progressive irregularities and multiple aneurysms within the lenticulostriate arteries. No changes were present in other cerebral vessels. There was no evidence of systemic disease. Brain biopsy was nonspecific. She was treated with high dose glucocorticoids with addition of cyclophosphamide for presumed primary CNS vasculitis. A third angiogram after steroids demonstrated complete resolution of aneurysms and irregularities. She remained well with no further evidence of disease at one year.

Conclusion: This is the first reported case of an immunosuppression responsive reversible vasculopathy with multiple fusiform aneurysms affecting only the lenticulostriate circulation.

\section{P-013}

The Canadian Activase for Stroke Effectiveness Study (CASES): final results

\section{Michael Hill (Calgary, Alberta), The CASES Investigators}

Background: Therapy for acute stroke using rtPA was approved in Canada in February 1999. The Canadian Activase for Stroke Effectiveness Study Group was formed to study the use of rtPA in
Canada in a 30-month post-marketing study (Feb 1999-June 2001).

Purpose: To both prospectively assess the safety of rtPA in the Canadian context, to examine whether the efficacy of rtPA for acute stroke, demonstrated in the NINDS trial, can be translated into effectiveness in routine clinical practice across Canada and to develop a prognostic index for patients treated with alteplase.

Methods: The CASES group is a collaboration among the Canadian Stroke Consortium (CSC), the Canadian Stroke Society, the Heart and Stroke Foundation of Canada, Hoffmann-La Roche Canada, the Canadian Stroke Network and physicians across the country. The study protocol was approved by the research ethics board at each centre. Demographics, stroke risk factors, blood pressure, biochemistry, hematology, and CT scans have been collected. NIHSS and mRS scores have been collected prospectively. CT scans are being scored centrally using ASPECTS. Outcomes have been monitored at discharge and at three months.

Results: 60 centres participated in the study. 1099 patients were recruited. The median NIHSS score is 15 (range 2-40). 30\% of patients have minimal or no neurological deficit (NIHSS 0-1) and $46 \%$ are independent (mRS $0-2)$ at 90 days. The symptomatic hemorrhage rate is $4.6 \%(95 \% \mathrm{Cl} 3.4 \%$ to $6.0 \%)$. The rate of protocol violations is $15 \%$, the majority of which are time window violations. Predictors of good outcome include baseline NIHSS score, baseline ASPECT score, age, baseline serum glucose, atrial fibrillation. Predictors of symptomatic intracerebral hemorrhage included baseline mean arterial blood pressure and baseline serum glucose.

Conclusions: CASES was a prospective evaluation of the effectiveness of rtPA in acute stroke. The symptomatic hemorrhage rate is low (4.6\%). Alteplase for ischemic stroke is safe and effective in Canada.

\section{P-014}

\section{Weather, chinook and stroke occurrence}

Thalia S. Field (Boston, USA), Michael D. Hill (Calgary, Alberta)

Background: Changes in weather and season have been linked to stroke occurrence. However, the association has been inconsistent across stroke sub-types. Calgary is a city in the Chinook belt and is subject to high variability in weather conditions.

Methods: We obtained hourly weather data over a five-year period from 1996-2000. We defined a Chinook according to the accepted definition. We reviewed administrative data to determine stroke occurrence. Stroke sub-types were strictly defined to maximize specificity of diagnosis. We compared stroke occurrence to Chinook onset and changes in temperature, relative humidity, barometric pressure and wind speed.

Results: No seasonal variation in overall stroke occurrence or occurrence by sub-type was evident. No relationship with changes in weather parameters was observed.

Conclusions: We found no association between weather changes and stroke occurrence. A cause and effect relationship between weather and stroke is dubious because of a lack of consistency across studies. 
Stroke prevention and the general practitioner - the value of a shared care model

\author{
J. Joubert, D. Ruth, C. Reid, L.B Joubert, S.M. Davis (Melbourne, \\ Australia)
}

Background: Shared care of stroke patients, after discharge from hospital, involving general practitioners in the modification of risk factors for stroke and the detection of post-stroke depression should improve outcomes for stroke survivors.

Methods: We shall describe the model and initial analysis of a shared care program for stroke patients at the Royal Melbourne Hospital, Australia.

The management plan focuses on two areas - medical risk factors for stroke and depression. Modifiable risk factors addressed include hypertension, dyslipidaemia, diabetes, cardioembolism, transient ischaemic attacks, smoking and obesity. A flow chart is employed and serial assessments carried out in a prospective audit at 3,6,9 and 12 month intervals.

Results: Initial analysis of the data suggests satisfaction with the model. Compliance with the management plan has been good. Initial outcomes regarding risk factor management and detection of depression will be discussed in detail.

Discussion: It is felt that a structured, "user-friendly" process of shared care, in accordance with recognized best practice guidelines, with prompting and follow-up from the acute stroke unit, will facilitate and enhance secondary stroke prevention, improve the early detection of anxiety and depression and result in consumer satisfaction.

\section{P-016}

\section{A survey of the state of readiness of acute stroke management across Canada}

\section{K. Khan (Edmonton, Alberta); P. Teal (Vancouver, British} Columbia); R. Kay (Hong Kong); N. Dean, M. Saqqur, A. Shuaib (Edmonton, Alberta)

Background: Since the approval of rt-PA ischemic stroke is treated as acute emergency. The success depends on the availability and quick access to various services.

Method: 71 members of CSC were sent the questionnaire. Data was collected on the use of rt-PA, immediate access to CT, stroke units, bed availability, stroke team, stroke hot line, referral policy, ambulance staff's awareness, stroke prevention clinic, training and hospital support for nurses, participation in the clinical trials, participants' level of satisfaction.

Results: $74 \%$ responded. $96 \%$ use IV rt-PA. $25 \%$ may give IA rtPA. $\mathrm{rt}-\mathrm{PA}$ is mainly administered in ER. $92 \%$ have immediate access to CT and ER physicians initiate it in $87 \%$ of the cases. Hot line and triaging by EMS is done in few centers. $32 \%$ have bypass policy. $38 \%$ have stroke units. Blocked beds in $28 \% .83 \%$ have stroke teams. EMS staff's awareness is $77 \% .43 \%$ have stroke prevention clinics and patients with TIAs are seen within one week of referral at $51 \%$ of the facilities. Training and support for the nurses is $34 \%$ and $36 \%$ respectively. Participation rate in trials is $85 \% .36 \%$ of respondents expressed satisfaction with their stroke programs.

Conclusion: IV rt-PA is widely available with variable facilities.
Is doppler ultrasound sufficient as the sole investigation before carotid endarterectomy?

\author{
C. Lovelock, P. Mitchell, J. Brown, D. Campbell, S. Davis \\ (Melbourne, Australia).
}

Background and Aims: Duplex imaging (DI) of the extracranial carotid arteries has been advocated as the sole imaging modality in carotid endarterectomy (CE) candidates. However this approach fails to identify potentially significant intracranial disease. Patients with intracranial atherosclerosis have a high risk of stroke and death. In this study, we aimed to identify the proportion of cases referred for $C E$ at our institution in whom the identification of intracranial disease could have altered clinical management.

Method: Two neuroradiologists, blinded to the clinical history, reviewed the films of $111 \mathrm{CE}$ candidates, predominantly of European background, who had undergone carotid angiography after screening DI. Intracranial stenoses greater than $50 \%$ of the luminal diameter, incidental aneurysms and non-atherosclerotic lesions were documented. Demographic and epidemiological data were collected.

Results: Out of 111,87 had a greater than $50 \%$ extracranial stenosis although two thirds were asymptomatic. Intracranial stenotic lesions were recorded in $29 \%$ of patients. Over two thirds were tandem lesions, distal to an extracranial stenosis. Aneurysms were found in $4.5 \%$ of patients.

Conclusion: Angiography demonstrated significant intracranial disease in nearly a third of the cases. The identification of this group allows more aggressive stroke prevention therapy and follow-up.

\section{P-018}

\section{A 53-year-old with cerebral venous sinus thrombosis}

Richard B. Lush, F.B. Maroum, Mark Stefanelli (St. John's, Newfoundland)

Background: Cerebral venous sinus thrombosis can present a diagnostic and therapeutic challenge to the most experienced of physicians.

Case report: We report a 53-year-old woman who presented with new onset generalized seizures. Her initial CT scan was reported as normal; however, in retrospect showed an empty delta sign. An MRI revealed a lesion in her right temporal lobe consistent with a glioma. Over the next six days she developed worsening neurological signs and a repeat $\mathrm{CT}$ scan showed a new left parietal lobe lesion. She was taken to the operating room for biopsy and intraoperatively was found to have superior sagittal vein thrombosis. In a patient postbrain biopsy with cerebral venous sinus thrombosis, should anticoagulation be used? A meticulous search of the literature failed to provide any direction. We decided to start low molecular weight heparin 24 hours postoperatively and she had almost complete neurological recovery.

Conclusion: It is important for physicians to be aware of the diverse clinical presentation and neuroimaging characteristics of cerebral venous sinus thrombosis so that unnecessary invasive procedures can be avoided. In patients post brain biopsy with cerebral venous sinus thrombosis one must be cautious with anticoagulation. In this case, low molecular weight heparin started 24 hours post craniotomy did not result in any clinical deterioration. 
P-019

The eyes have it! CT assessment of conjugate eye deviation in acute stroke

S.C. Morgan (Kingston, Ontario); J.E. Simon, J.H.W. Pexman, M.D. Hill, A.M. Buchan (Calgary, Alberta)

Background: Conjugate eye deviation (CED) helps lateralize the side of hemispheric lesions in stroke but suffers from poor interobserver agreement. We investigated: 1) the use of CT in assessing $\mathrm{CED} ; 2)$ the prevalence of deviation that lateralizes the brain lesion; and 3) whether the presence of CED correlates with initial stroke severity.

Methods: CT scans of 115 hyperacute stroke patients were independently reviewed by four raters. Raters, blinded to clinical information, classified gaze as: 1) undeviated, 2) rightward CED, or 3) leftward CED. When no CED was seen, the raters indicated the side of any lone abducting eye (LAE), which a pilot study had found to be a lateralizing sign.

Results: Assessment of gaze was possible in 107 scans. Kappa scores were good to excellent. Eye deviation was more common on CT (40\% CED and 30\% LAE) than has been reported on clinical exam (16-28\%). The positive predictive value of gaze deviation for correctly lateralizing lesion side was high: 0.91 for CED and 0.85 for LAE. The presence of CED did not correlate with presenting NIHSS.

Conc/usion: The assessment of eye deviation on acute stroke head CT scans is an excellent clinical adjunct that reliably indicates the ischemic hemisphere.

\section{P-020}

Intravenous rt-PA for acute stroke: results from 76 consecutive patients

\section{Mouradian G. Jickling, A. Shuaib (Edmonton, Alberta)}

Background: Self auditing and patient follow-up may further improve the appropriate use of intravenous $\mathrm{rt}$-PA for acute stroke.

Method: Prospective stroke database and follow-up for outcome after receiving IV rt-PA at a tertiary care center.

Results: 76 patients received IV rt-PA from January 1999 to July 2001. Survivors were followed-up three to 24 months after treatment. Mean age was 72 (range 34-96). Mean NIHSS score at baseline presentation was 14 (range 4-27). Mean time from onset to emergency department (ED) arrival was 70 minutes (range 30-155). Time from ED to brain CT was 28 minutes (range 7 to 35 ). CT to needle time was 49 minutes (range 12-59). Door to needle time was 76 minutes (range 20-95 minutes.). Onset to needle time was 147 minutes (range 63-185). Ten patients died (13\%) within three months. Three deaths were non-stroke related. Mean follow-up NIHSS score was 4 (range 0-25). Barthel index score was 85 to 100 in $74 \%$ of the patients. Modified Rankin score was 0 or 1 in $66 \%$ of surviving patients.

Conclusion: Our results are comparable to NINDS trial.
P-021

Acute hyperglycemia adversely affects stroke outcome: an MR imaging and spectroscopy study

M. Parsons, PA. Barber, P. Desmond, T. Baird, B. Tress, S. Davis (Melbourne, Australia).

Background: In animal stroke models, hyperglycemia increases brain lactic acidosis and conversion of penumbral tissue to infarction. These relationships have not been explored in humans.

Methods: Thirty acute stroke patients were prospectively evaluated with sub-24 hour, day-3, and day-90 diffusion- and perfusion-weighted MRI, and acute blood glucose measurements (mean 25 minutes before acute MRI). Patients with hypoperfused 'at-risk' tissue were identified by acute perfusion>diffusion lesion mismatch. Acute and subacute MR spectroscopy was also performed to assess the relationship between acute blood glucose and lactate production in the ischemic region.

Results: In the 18 of 30 patients with perfusion $>$ diffusion mismatch, acute hyperglycemia correlated with increased conversion of acute mismatch tissue to infarction, greater final infarct size, and a worse functional outcome. These correlations were independent of baseline stroke severity, lesion size, and diabetic status. Furthermore, acute hyperglycemia was associated with an increase in acute-subacute lactate level in the ischemic region, which in turn was independently associated with increased acute mismatch tissue progressing to infarction.

Conclusions: Acute hyperglycemia adversely affects stroke outcome by increasing brain lactate production, which facilitates progression of hypoperfused 'at-risk' tissue into infarction. These findings support the need for randomized controlled trials of aggressive glycemic control in acute stroke.

\section{P-022}

Vertebral artery dissection with basilar artery occlusion presenting with bilateral upper extremity paresthesias and weakness

\section{A.H.Rajput, C. Toth (Saskatoon, Saskatchewan)}

Background: While headache, neck pain and neurological deficits are important clinical features of vertebral artery dissection, bilateral upper extremity paresthesias and weakness has not been described in the literature.

Methods: A 38-year-old man noted sudden onset neck pain associated with transient weakness and paresthesias of both hands with mild vertigo followed by right-sided headache. He had minor head trauma three weeks previously but his history was otherwise unremarkable.

Results: Neurological exam and head CT on admission were both normal. Two days after admission he developed right face and arm weakness with nystagmus and anticoagulation was started. Magnetic resonance angiography and imaging (MRA and MRI) revealed absence of the basilar artery and the proximal portions of both intracranial vertebral arteries associated with an acute left pontine infarct. Angiogram showed almost complete occlusion of the basilar artery with dissection of the right vertebral artery and a hypoplastic left vertebral artery. He was treated with warfarin and made a good recovery.

Conclusions: The most common clinical presentation of 
vertebral artery dissection is neck pain, headache, and neurological deficit. We speculate that transient bilateral arm weakness and paresthesias in this patient was secondary to either bilateral pontine or anterior cervical cord ischemia.

\section{P-023}

Posterior leucoencephalopathy syndrome following intrathecal chemotherapy with arterial vasospasm on MRA

S.J.Read, R.D. Henderson, T. Rajah, A. Nicol (Brisbane, Australia)

Background: Posterior leucoencephalopathy syndromes (PLES) are typically reversible and associated with hypertension. Reported causes include eclampsia, immunosuppression and chemotherapy. However, not all cases are reversible or associated with hypertension. We describe PLES following intrathecal (IT) chemotherapy associated with vasospasm on MRI.

Case report: A 54-year-old lady developed bilateral cortical blindness, ocular apraxia and astereognosis approximately one week after receiving high-dose IT methotrexate and cytarabine. At the time, she was cytopenic and febrile but was not significantly hypertensive. MRI showed extensive areas of increased signal on T2-weighted images in the white matter of the posterior parietooccipital lobes bilaterally, with high signal on diffusion-weighted images and a restricted apparent diffusion coefficient, consistent with cytotoxic edema. Extensive irregularities were present in large arteries of both the anterior and posterior circulation on MRA. Three weeks later, neurological deficits and parenchymal MRI changes had improved but not resolved. Resolution of the vascular irregularities on MRA was consistent with vasospasm.

Conclusions: In this case, PLES followed IT chemotherapy in the absence of hypertension. Parenchymal MRI changes were largely irreversible cytotoxic edema, whereas previously reversible changes consistent with vasogenic edema have been reported. Vasospasm has been described in PLES with hypertension (eg. in eclampsia). Vasospasm triggered by IT chemotherapy appears to be another cause of PLES.

\section{P-024}

\section{Does a dedicated stroke ultrasound service improve reliability} of Doppler in carotid disease?

N. Dean, H. Romanchuck, M. Saqqur, K.N. Khan, M. Moratoglu, N. Amir, A. Shuaib (Edmonton, Alberta)

Introduction: Doppler ultrasound is a useful screening tool to assess the degree of carotid stenosis. There are recent reports that the technique may be inaccurate in over $28 \%$ of patients. We evaluated the accuracy of ultrasound in a dedicated stroke clinic.

Methods: We reviewed the charts of patients with a recent transient ischemic attack or a mild stroke and who had ultrasound performed in our stroke prevention clinic. All patients subsequently underwent cerebral angiography. Majority of these patients had greater than $50 \%$ stenosis on ultrasound. In a smaller group with less than $50 \%$ stenosis on ultrasound, angiography was performed for diagnostic reasons. In the final group, angiography was performed to confirm arterial occlusion.

Results: Seventy-two patients were included in the study. Group I had 50 patients with ultrasound measured stenosis of $50-99 \%$. On review of angiograms, only one patient was considered to be unsuitable for carotid endarterectomy. Group II had 19 patients with ultrasound measured stenosis of less than $50 \%$. None of these patients had a stenosis of greater than $50 \%$ on angiography. In Group III there were five patients in whom ultrasound showed complete occlusion of carotid artery, confirmed by angiography.

Conclusions: Doppler ultrasound has a high accuracy rate in the setting of a dedicated stroke clinic.

\section{P-025}

Stroke risk in patients undergoing coronary artery bypass graft surgery

\section{R. Tang-Wai, R. Cote (Montreal, Quebec)}

Background: The goals of this study are (1) to determine the incidence of stroke in a consecutive cohort of patients submitted to CABG, and (2) to determine whether carotid artery stenosis alone or in conjunction with other risk factors is predictor of perioperative stroke.

Methods: This study involved a retrospective chart review of 563 consecutive patients who had undergone CABG between April 1999 and October 2000 at the Montreal General Hospital. Age, sex, history of diabetes, hypertension, hypercholesterolemia, smoking, stroke, cardiac arrhythmia, degree of carotid artery stenosis, pump time, and intraoperative use of vasopressors were examined. The primary endpoint was stroke occurring in the $\mathbf{3 0}$ day postoperative period.

Results: To date, 103 cases were reviewed. The characteristics are as follows: mean age 67.8 years, males $71 \%$, diabetes $30 \%$, hypertension $80 \%$, smoking $39 \%$, hypercholesterolemia $57 \%$, atrial fibrillation $13 \%$, and prior stroke $3 \%$. Carotid duplex was performed preoperatively in 15 patients: five patients had high grade stenosis of greater than $70 \%$; five patients had moderate stenosis of 15 to $70 \%$; and five patients had low grade stenosis of less than $15 \%$. In total, six patients suffered ischemic strokes (incidence of $6 \%$ ): two patients had bilateral carotid artery stenosis of greater than 70\%; one patient had low grade stenosis; and in three patients no studies were performed. Further data will be included for final presentation.

Conclusion: The incidence of stroke in the perioperative period of $\mathrm{CABG}$ is low in our institution and comparable to that reported in the literature.

\section{P-026}

Outcome analysis of arteriovenous malformations treated with stereotactic radiosurgery at the British Columbia Cancer Agency

\section{K. Tankel, M. McKenzie, B. Toyota, B. Clarke, J. Robar}

(Vancouver, British Columbia)

Purpose: To perform a retrospective outcome analysis of the first 50 arteriovenous malformation patients treated with stereotactic radiosurgery at the British Columbia Cancer Agency (BCCA).

Methods and Materials: BCCA and Vancouver Hospital patient files and dose distributions were surveyed. Patients were stratified for age, sex, lesion location and previous treatment. Target volume, 12 Gy volume as well as maximum and mean doses were recorded. All reversible and irreversible neurological deficits detected in followup, as well as all radiological sequelae were recorded and graded. 
Obliteration rates as well as complication rates will be reported and compared to the literature.

\section{P-027}

Migraine and stroke: cause, consequence or coincidence?

\section{N. Vujovic-Zotovic, J.W. Norris (Toronto, Ontario)}

Background: Migraine is said to cause stroke or TIA when the typical aura precedes the neurological deficit, but it may be unrelated, or sometimes stroke appears to trigger a migraine attack.

Methods: To determine this relationship we evaluated the medical records of 1031 consecutive patients referred to our Stroke Prevention Clinic with diagnosis of TIA or stroke.

Results: 91/1031 (9\%) had migraine and 36 of these had a visual aura only (misdiagnosed as TIA). The remaining 55 had neurological symptoms: 31 of these had TIAs with visual aura, 14 had TIAs with no visual symptoms and in 10 patients with stroke, six were preceded by visual auras. Migraine appeared to be the cause of TIA or stroke in $16 / 24(67 \%)$, coincidental in five $(21 \%)$ and consequence in three $(12 \%)$ patients.

Conclusion: Our data suggest that when migraine occurs with stroke it is usually causal, but may be coincidence or consequence.

\section{P-028}

Correlating $18 \mathrm{~F}$-fluoromisonidazole (FMISO) imaging in acute stroke with cerebral blood volume (CBV) and cerebral blood flow (CBF) measured with magnetic resonance imaging (MRI)

P. Wright, G. Donnan, T. Phan, R. Markus, A. Falcao, D. Reutens

(Melbourne, Australia).

Background: Preliminary results show that FMISO trapping occurs in tissue identified by MRI as at risk of infarction. We examined the MRI perfusion indices in tissue compartments defined by DWI, T2-weighted MRI, and FMISO PET.

Merhods: Nine patients underwent MRI and PET scanning in acute stroke, and 90 days later for identification of diseased tissue.

$\mathrm{CBV}$ and $\mathrm{CBF}$ values were determined in tissue compartments defined by 1: the FMISO lesion (metabolic penumbra), 2: the T2weighted lesion (final infarct), 3: the diffusion weighted lesion (estimates acute ischaemic core).

Results: CBF was 26-30\% lower in FMISO labelled regions that were also abnormal on DWI than in a) surviving, b) infarcting areas of FMISO uptake, or c) DWI lesions not labelled with FMISO (NSS). CBV was 10\% higher in FMISO labelled areas that later infarcted than in contralateral gray matter. CBV was $26 \%$ lower in DWI affected regions also labelled with FMISO than in tissue with evolving infarction $(\mathrm{p}=0.15)$.

Conclusion: FMISO lesions include tissue with a) reduced perfusion and abnormal water diffusion that may represent an ischaemic core, b) reduced $\mathrm{CBF}$ and elevated $\mathrm{CBV}$ that infarcts, and c) reduced $\mathrm{CBF}$ but minimally affected $\mathrm{CBV}$ that is viable.

\section{P.029}

18F-fluoromisonidazole (18F-FMISO) uptake in acute stroke detects tissue at risk of infarction

P. Wright, G. Donnan, T. Phan, R. Markus, A. Falcao, D. Reutens

(Melbourne, Australia)

Background: The PET tracer 18F-FMISO is trapped in hypoxic tissue and identifies the metabolic penumbra following acute stroke. We studied the pattern 18F-FMISO trapping in ischemic stroke within tissue compartments defined by MRI diffusion and T2weighted imaging. MRI has not previously been used to identify tissue compartments within 18F-FMISO studies.

Methods: Nine patients were studied. MRI and 18F-FMISO scans were performed within 24 hours (mean 11 hours) of stroke onset (9/9) and 90 days later (7/90). The region of FMISO uptake was examined for its relationship to 1) the final infarct on the T2 scan at 90 days; 2) the diffusion weighted lesion.

Results: $16 \%$ of tissue with FMISO uptake had abnormal DWI, although $82 \%$ of the DWI lesion was separate from this tissue. Another 23\% of tissue with FMISO uptake had late infarction. Of the tissue at risk defined as DWI negative but T2 positive, $40 \%$ was identified by FMISO uptake. The sensitivity of this test for predicting penumbral infarction is 40 percent. The specificity for predicting evolution to infarction is 23 percent, and for predicting final infarction is 32 percent.

Conclusion: FMISO is a good predictor of the evolution of infarction.

\section{P-030}

Temporal profile of cytokines in patients with vertebrobasilar transient ischemic attack

K.M.Yoo, B.G. Yoo, K.T. Ji, K.S. Kim (Busan, Korea)

Background: Until recently, cytokine changes in transient ischemic attack (TIA) have not been studied. The purposes of this study are to evaluate the temporal profile of the inflammatory response in vertebrobasilar TIA and to compare the inflammatory response between TIA and infarction.

Methods: We serially (at the timing of admission and at days 1 and 5 after symptom onset) measured the serum IL (interleukin)- 6 and IL- 10 in 47 patients (vertebrobasilar TIA $(n=14)$, vertebrobasilar territory infarction $(n=12)$, and lacunar infarction $(n=21)$ ) and 13 normal controls. Cytokines were measured by sandwich ELISA method.

Results: IL-6 level at day 1 was significantly elevated compared with normal group $(\mathrm{p} \leq 0.01)$ and was the highest in vertebrobasilar TIA. But, IL-6 level between vertebrobasilar TIA and two infarction groups did not show any differences. IL-10 in vertebrobasilar TIA was significantly higher than normal group at all time points $(\mathrm{p} \leq 0.05)$. IL-10 at day 5 was significantly elevated in two infarction groups than vertebrobasilar TIA ( $\mathrm{P}<0.05-0.01)$.

Conclusions: As the result of this, it strongly suggests that cytokine cascade do occur in TIA. TIA also has the presence of rapid pro-inflammatory reaction. The pathophysiology of TIA is different from infarction in the anti-inflammatory response. 


\section{DEMENTIA}

P-031

\section{Predictors of caregiver burden from the Canadian outcomes study in dementia (COSID)}

\section{Borrie, M. Smith (London, Ontario), the COSID Investigators (national)}

Background: Increasing caregiver burden is a common precursor to patient institutionalization of people with dementia. This analysis was a preliminary examination of the predictors of change in caregiver burden in a community-dwelling cohort of caregivers.

Method: Baseline and six-month data was obtained from the Canadian Outcomes Study in Dementia (COSID), a national, prospective observational study $(n=182)$. Changes in caregiverburden were assessed using the Zarit Burden Interview (ZBI). Independent variables included the Neuropsychiatric Inventory (NPI), Modified Mini Mental Status Exam (3MS), and Functional Autonomy Measurement System (SMAF). Correlations were examined with a multiple regression model using changes in scores from baseline to month six.

Results: Change in caregiver burden (ZBI) was most strongly associated with behaviour changes (NPI) in persons with dementia $(p=0.002)$. Caregivers of male patients and those using support groups were more likely to have higher burden change scores ( $p=$ 0.021 and $p=0.008$, respectively). Changes in cognition (3MS) and self-care ability (SMAF) were not significantly associated with a change in burden score.

Conclusions: The results highlight the importance of effective management of the behaviour disturbances associated with dementia.

\section{$\mathbf{P - 0 3 2}$}

\section{Clinical predictors of falls in two specialized Alzheimer care} units

\section{R. Camicioli, S. Foster, L. Licis (Edmonton, Alberta)}

Background: Falls are common in Nursing Homes and in patients with Alzheimer's disease. Falls predispose to injuries, decreased independence and death. Few studies have focussed on factors related to fall-risk in specialized Alzheimer Care Units (ACU).

Methods: Cross-sectional study comparing clinical features, Morse Falls Scale risk and quantitative gait parameters in $\mathrm{ACU}$ residents stratified by falling history. Quantitative gait measures including speed, cadence, stride length and variability in these parameters were measured using a GaitRite carpet.

Results: Fallers $(n=18)$ did not differ from non-fallers $(n=22)$ with respect to age, MMSE, activities of daily living, dementia rating scale score (CDR), Modified Ischemic Score or behavior (Neuropsychiatric Inventory). Fallers had higher Morse Falls Scale $(p<0.0001)$ and extrapyramidal (modified Parkinson's Disease Rating Scale, $p=0.003$ ) scores. Neuroleptic use did not differ. Among the quantitative measures, only self-selected gait speed differed between fallers and non-fallers ( $\mathrm{p}<0.05$ ).

Conclusions: Fallers with Alzheimer disease differed from nonfallers on simple measures of falls risk. These findings offer hope for predicting future fallers and for the development of interventions to prevent falls. The strong association with extra-pyramidal signs highlights the importance of minimizing neuroleptic use in people with advanced Alzheimer disease.

\section{P-033}

\section{Effectiveness of dementia day care centres in Hong Kong,} China

\section{H. Chiu, L. Lam (Hong Kong)}

Background: Dementia care has just started to develop in Hong Kong China and four dementia day care centres (DDCC) were set up in 1999. This study aimed to examine the effectiveness of this pilot project.

Method: Elderly patients with dementia attending the DDCCs (77 subjects) were assessed with the mini-mental state examination, Activities of daily living, Instrumental Activities of daily living scales, Neuropsychiatric Inventory and Cohen-Mansfield Agitation Inventory. Carers of the subjects were assessed with the Zarit Burden Interview and General Health Questionnaire. Measurements were taken at baseline and at four month interval until discharge. A control group of elderly with dementia not attending day care centers (44 elderly) were assessed with the same instruments.

Results: Compared to controls, subjects attending DDCC had more significant reduction in their behavioural and psychological symptoms and better preservation of their functional level. Significant reduction of scores on burden scale and General Health Questionnaire were found in the carers of subjects of DDCC but not for carers of controls. However, there was no delay in the rate of admission to residential services in the subjects compared with controls.

Conclusions: This study showed that DDCCs had beneficial effects on Chinese elderly with dementia and their carers.

\section{P-034}

Serial evaluation of asymptomatic mild cognitive impairment (MCI) using computerised testing

\section{D.G. Darby, P. Maruff, A. Bush, A. Collie (Melbourne, Victoria)}

Background: Computerised internet-deliverable diagnostic tools may allow detection of early $\mathrm{MCI}$ prior to significant disability, and serial monitoring of subtle cognitive decline. We evaluated subjects with asymptomatic $\mathrm{MCI}$ serially over 12 months using a brief novel computerised cognitive screening test (CogState). We aimed to determine whether CogState could (i) differentiate $\mathrm{MCI}$ from controls consistently, and (ii) characterise $\mathrm{MCI}$ neuropsychologically.

Methods: 81 subjects from a longitudinal study of healthy aging volunteers (studied since 1995) were tested with CogState and neuropsychological tests at $0,1,3,6$ and 12 months. There were 61 normal $(68 \pm 6.3$ years $)$ and 20 asymptomatic $\mathrm{MCl}(63 \pm 7.8$ years $)$ subjects based on longitudinal evaluation (eg. impaired CERAD word list delayed recall task). Analyses used parametric and multivariate methods.

Results: MCI subjects were differentiable from controls throughout follow-up. Group differences were reliably present particularly in variables measuring declarative memory. In addition, specific analyses showed no attentional dysfunction, and no speed/accuracy trade-off strategies.

Conclusions: $\mathrm{MCI}$ can be detected and followed serially in 
asymptomatic community divelling individuals, raising the potential for its use in the wider community. In addition, deficits can be differentiated from less specific patterns by the differential involvement of declarative memory.

\section{P.035}

Lumbo-peritoneal shunt as a diagnostic aid in the management of nornal pressure hydrocephalus: the Oregon Aging and Alzheimer Center experience

D. Erten-Lvons, (Portland, USA); R. Camicioli, (Edmonton, Alberta); J. Kaye, L. Silbert, E. Frank, J.F. Quinn, (Portland, USA)

Background: The selection of patients for ventriculoperitoneal (VP) shunting for normal pressure hydrocephalus (NPH) is challenging, due to the poor specificity of diagnostic criteria and the morbidity of VP shunting. Lumboperitoneal (LP) shunting has been proposed as a diagnostic aid, permitting a prolonged trial of cerebrospinal fluid (CSF) drainage while minimizing morbidity.

Methods: The database of the Oregon Alzheimer's Disease Center from 1991-2001 was searched for all subjects in whom NPH was listed as a keyword, and case histories were obtained by chart review.

Results: Twenty subjects were identified. Four had only a large volume lumbar puncture, and two were referred directly for VP shunt. Fourteen subjects received an LP shunt. Over 3-12 months of follow-up there were no serious complications. Nine were referred for VP shunting and all had excellent results, with improved gait and mentation for at least several weeks. One case was not referred for VP shunting in the absence of improvement with LP shunting. Four were lost to follow-up.

Conclusions: LP shunting is a low morbidity procedure which may help to distinguish subjects who are likely to benefit from VP shunting from those who are not. Prospective clinical trials assessing the use of LP shunting for NPH will require multi-center collaboration.

\section{P-036}

\section{An unusual case of Creutzfeldt-Jakob disease}

A. Gagnon, F. Emond, M. Thibault, F. Bédard, P.V. Gould, J.-P. Bouchard (Quebec City, Quebec)

Background: Creutzfeldt-Jakob disease (CJD) is a degenerative disease of the brain which is classically defined as a triad of rapidly progressive dementia with myoclonic jerks and characteristic EEG abnormalities. However, the spectrum of clinical presentations for CJD can be varied, including rare stroke-like presentations. We describe a case of CJD with an unusual presentation, evolution and imaging.

Case report: A 69-year-old woman presented with a history of confusion, ataxia and word-finding difficulties that had started on waking up in the morning 10 days earlier. She was admitted with a provisional diagnosis of stroke. CT scan on admission was normal. However, over the next three weeks a rapid deterioration occurred, with progressive deficits of most cortical functions, including a frontal syndrome progressing up to akinetic mutism. Myoclonic jerks were noted. On MRI, diffusion-weighted imaging (DWI) showed slight hyperintensities of the basal ganglia on the left, and a left parietal cortical hyperintensity. The initial EEG showed generalized slowing without epileptiform discharges. About four weeks later, periodic discharges with a triphasic pattern and burstsuppression activity were seen. CSF was positive for the 14-3-3 protein. The patient died less than six weeks after the onset of her symptoms; post-mortem examination showed no macroscopic evidence of stroke. Microscopic examination is pending.

Discussion: This CJD patient presented with stroke-like symptoms; only two similar reports can be found in the literature. Likewise, her fulminant evolution is unusual for the disease. Finally, the DWI hyperintensities of the basal ganglia are usually symmetrical in CJD.

Conclusion: This case of CJD illustrates the wide clinical and radiological spectrum of the disease. Clinicians should be aware of the many faces CJD can take.

\section{P-037}

Acute fulminating adult-onset subacute sclerosing panencephalitis in a 49-year-old man

\section{A. Gagnon, R.W. Bouchard (Quebec City, Quebec)}

Background: Subacute sclerosing panencephalitis (SSPE) is a rare, slow viral infection caused by defective measles virus. It is characterized by progressive mental deterioration associated with motor impairment and prominent myoclonus. SSPE follows measles infection by several months to several years, and usually affects children or young adults. In about $10 \%$ of all cases, the disease can progress rapidly and lead to death within a few months. The oldest previously reported fulminating case was a 34-year-old woman.

We report the case of a 49-year-old man with clinical, laboratory and pathological evidence of SSPE and review adult-onset SSPE.

This 49-year-old right-handed man was referred to neurology in March 2001 because of recent behavioural changes and progressive cognitive impairment over the past few months. Past medical history was unremarkable except for an episode of measles in his childhood. Mental status examination revealed visual hallucinations, poor attention and concentration, disorientation, perseverance, anomia, acalculia and ideational apraxia. Basic neurological exam showed bilateral myoclonic jerks with hyperreflexia with bilateral Babinski sign. Ophthalmic exam revealed bilateral macular swelling and papilledema. Extensive laboratory studies were unremarkable. EEG showed periodic sharp and slow-waves discharges. MRI showed diffuse T2 signal hyperintensity in periventricular and subcortical white matter with bilateral insular involvement. Serum measles IgG antibody titer was positive. CSF examination revealed 40 monos, proteins at 92 and IgG at $29 \%$. There were four oligoclonal bands. CSF anti-measles antibody titers were highly positive. 14-3-3 protein was faintly present. Cerebral biopsy revealed encephalitis and measles RT-PCR ARN was positive. Omaya reservoir was inserted and high-dose intrathecal interferon alpha with oral isoprinosine were administered. Despite transient subjective improvement, the patient continued to deteriorate, became bedridden and died in June 2001 .

Conclusion: To our knowledge, this is the oldest case of SSPE reported to this date in the literature. Dementia with rapid decline is a potential presentation of SSPE. Treatment has been so far disappointing though some mild benefits have been reported. The importance of providing an early diagnosis will be particularly relevant as more effective treatments become available. 
Variations in the pattern of neuropsychological deficit in mildmoderate Alzheimer's disease

\section{E. Storey, R. Hester (Melbourne, Australia)}

Background: A number of recent studies have documented asymmetric involvement of language and visuospatial functions in Alzheimer's disease (AD), implying hemispheric asymmetry. More contentious has been the existence of a frontal variant of $A D$, although case studies, small series and factor analyses have all supported anteroposterior asymmetry of involvement as well.

Methods: Thirty-six patients attending a memory clinic received a diagnosis of AD (MMSE 15-27) after assessment by a neurologist using the CERAD protocol, psychogeriatrician, and neuropsychologist for further testing if required, as well as neuroimaging and an OT home visit. Diagnosis was by consensus, using NINCDS-ADRDA criteria. Hierarchical cluster analysis was performed on z-scores from the CERAD shortened Boston Naming Test, CERAD constructional praxis, and Stroop test (of cognitive inhibition), and indicated three main clusters. K-means cluster analysis was then performed, and test scores at the centre of each cluster calculated.

Results: The three clusters identified were: naming/construction affected equally ( $n=23$ ); naming disproportionately affected $(n=5)$; "frontal" (cognitive inhibition) disproportionately affected $(n=8)$.

Conclusions: This analysis supports the concept of asymmetric neuropsychological involvement in $\mathrm{AD}$, and particularly the existence of a predominantly "frontal" variant. The Stroop test (Victoria version) may be a useful brief addition to the CERAD neuropsychological protocol.

P-039

Executive dysfunction in spinocerebellar ataxia type 1 (SCA 1) and Friedreich's ataxia (FA) - preliminary results

\section{R. Hester, K. Tuck, M. Delatycki, E. Storey (Melbourne, Australia)}

Background: We hypothesize that the extent of executive dysfunction will correlate with the degree of ataxia in cerebellar but not spinal ataxias. As part of an ongoing study, we report preliminary results for SCAl $(n=11), F A(n=10)$ and controls $(n=16)$.

Methods: Ataxia severity was judged using ICARS (International Cooperative Ataxia Rating Scale), and a neuropsychological battery comprising the NART, MMSE, WCST, verbal fluency WAIS-3 similarities, Trails, Stroop, BADS card shift and zoo map tests, and various Lurian motor control tests was administered.

Results: The three groups were not significantly different for age, education, or NART-estimated IQ. The SCAl group differed significantly from controls on Stroop Interference time, card shift, and three Lurian tasks, while WAIS-3 similarities and trails interference almost reached significance. FA subjects did not differ from the other two groups on any of these measures. All groups differed significantly on the ICARS, with FA subjects most severely affected. Most neuropsychological measures correlated with ICARS scores. Exceptions included the card shift, similarities and 2/3 Lurian tasks.

Conclusions: Executive dysfunction was demonstrated in SCA 1 but not FA. This is not confounded by poor motor performance. These preliminary findings are consistent with our hypothesis.

\section{Antioxidant protection from HIV-1 gp120 induced neuroglial toxicity}

K.A. Walsh, J. Crukley, J. Megyesi, J.X. Wilson, A.W. Gelb, R.R. Hammond (London, Ontario)

Background: Mechanisms underlying HIV-1 gp120 associated neuroglial toxicity remain unresolved but oxidative injury is likely a relevant factor. In-vitro exposure of primary human CNS tissue cultures to gp/20 leads to a simplification of neuronal dendritic elements as well as astrocyte hyperplasia and hypertrophy similar to the neuropathology of HIV-1 in vivo. Gpl20 and proinflammatory cytokines have been shown to upregulate iNOS, an important source of endogenous oxidation through the production of NO.

Methods: CNS cultures are derived from 16 to 18 week gestation postmortem fetal brain. Tissue is dissociated to a single cell suspension and maintained as monolayers on polyornithine-laminin coated culture ware and as aggregates in uncoated flasks. Cultures are grown for four weeks before exposure to gp 120 . Neuroglial antigens are examined by immunohistochemistry and immunofluorescence for confocal scanning laser microscopy.

Results: Recent experiments have shown that iNOS is markedly upregulated from undetectable levels at baseline and that this upregulation is abolished by preincubation with ascorbate.

Conclusions: This suggests that ascorbate supplementation supports neuroglial antioxidant reserves depleted by the effects of gp120 exposure, preventing the deleterious upregulation of iNOS. Further experiments will examine changes in the transport and metabolism of ascorbate and other antioxidants in association with gp120 exposure.

\section{NeUroscience}

\section{P-041}

The effect of liposomal formulations of tacrolimus and rapamycin on grafted cell survival in hemiparkinsonian rats

\section{A.Y. Alemdar, I. Mendez (Halifax, Nova Scotia)}

Background: The immunosuppressive drugs tacrolimus (TAC) and rapamycin (RAPA) have both been found to have neuroprotective effects on dopaminergic neurons. The purpose of the present study was to investigate whether liposomal formulations of these drugs would retain these neuroprotective properties.

Methods: 6-hydroxydopamine lesioned rats were transplanted with 800,000 fetal rat ventral mesencephalic cells and randomly divided to one of four groups. Group I received transplants containing cells only; Group 2 received a cell suspension containing $0.68 \mu \mathrm{M}$ liposomal RAPA (LRAPA); Group 3 received a cell suspension containing $2.0 \mu \mathrm{M}$ liposomal TAC (LTAC); and Group 4 received a cell suspension containing a liposomal formulation of both $0.68 \mu \mathrm{M}$ RAPA and $2.0 \mu \mathrm{M}$ TAC (LRAPATAC). Transplanted rats were assessed for rotational behavior three and six weeks posttransplantation. Cell survival was assessed using tyrosine hydroxylase (TH) immunohistochemistry.

Results: A significant reduction in amphetamine-induced rotational scores was seen in all groups, and all animals were found to have surviving THir grafts. However, the animals receiving a cell 
suspension containing either LTAC or LRAPATAC were found to have significantly more surviving THir cells than the group receiving cells only.

Conclusion: This study shows the feasibility of using liposomal formulations of neuroimmunophilins to improve graft survival in neural transplantation for Parkinson's disease.

\section{P.042}

Behavioural improvement with low dose dopaminergic grafts in the 6-OHDA rodent model of Parkinson's disease

\section{L.E. Bartlett, D. Sadi, I. Mendez (Halifax, Nova Scotia)}

Background: The positive behavioural effects of neural transplantation in Parkinson's disease are often directly attributed to the number of cells in a graft. The possibility of using smaller grafts would decrease the demand for fetal tissue, decrease structural damage to the host striatum (STR) and decrease the possibility of viral transmission to the patient due to multiple fetal donors. This study demonstrates that a low dose graft has the potential to promote behavioural recovery equal to a high dose graft over time.

Methods: Twelve rats received either 50,000 or 400,000 fetal ventral mesencephalic cells implanted into the STR. Twelve weeks post-transplantation, animals were perfused and microtome sections were stained for tyrosine hydroxylase (TH). Fiber outgrowth, cell diameter, cell survival and cell counts were calculated.

Results: The high dose group demonstrated a more rapid recovery initially, however, after six weeks, there was no significant difference in rotational behaviour scores between the two groups. Cell counting revealed a mean of 363.4 surviving TH-positive cells in the low dose group, compared to 1565 in the high dose group. The low dose group had a higher percentage of fiber outgrowth, higher TH-positive cell survival and increased cell diameter.

Conclusions: This study demonstrates that over time, a low dose dopaminergic graft has the capability of eliciting the same functional effect as a high dose graft over time.

\section{P.043}

Expression of heat shock protein 27 (hsp27) in the ganglion cell layer of the neonatal rat retina

\section{E. Hawkes, A.M. Krueger-Naug, R.W. Currie, D.B. Clarke \\ (Halifar, Nova Scotia)}

Background: The small heat shock protein hsp27 has been shown to protect neuronal cells from undergoing apoptosis in the context of cell stress, and recent ivork in our laboratory has indicated a role for hsp27 in the retina of the adult rat. The purpose of this work, therefore, is to examine the developmental expression of hsp27 in the retina.

Methods: 52 albino rats (four at each of thirteen postnatal time points between the postnatal day 0 [P0] and P24) were perfused. Eyecups were dissected, fixed, cut ( 30 micron sections), and reacted with an antibody that recognizes hsp27. Cell profiles were counted in a blinded and random fashion, and appropriate statistical analysis was performed on the data.

Results: Preliminary results indicate that hsp27 is not expressed at early (P0-P3) or later (P15 onwards) periods, but peaks (upivards of 4,000 hsp27-positive retinal ganglion cells (RGCs) per retina) sharply at P9-P12. This correlates with RGC innervation of the superior colliculus, thus suggesting a role for hsp27 in the fate of these RGCs.

Conclusions: We have demonstrated that the developmental expression of hsp27 in the retina peaks in retinal ganglion cells at P9-12. Our results indicate that hsp27 may have an important role in the survival and target innervation of developing retinal ganglion cells.

\section{P-044}

\section{IL-5 therapy ameliorates experimental allergic neuritis (EAN)}

S. Hodgkinson, N. Carter, G. Tran, X.Y. He, M. Killingsworth, B. Hall (Liverpool, Australia)

Experimental allergic neuritis (EAN), an animal model for Guillain-Barré Syndrome (GBS) is a T cell mediated demyelinating disease. In this study, we examined the effectiveness IL-5 to reverse established disease and to prevent the onset of clinical symptoms. EAN was induced in Lewis rats by the inoculation of bovine peripheral myelin and complete Freunds adjuvant. Daily intraperitoneal injections of $1 \mathrm{~L}-5$ from induction of disease and from onset of clinical symptoms were administered. IL-5 therapy from the onset of clinical symptoms ameliorated disease. However, when administered from induction to onset, it was ineffective. Our results demonstrated the cytokine IL-5 could be used successfully as a therapy in immune-mediated demyelinating diseases.

\section{P.045}

\section{Neuronal stem cells migrate into striatum in a rat model of Huntington's disease}

\section{Kim, B.S. Jeon, (Seoul, Korea); S.U. Kim, (Vancouver, British Columbia).}

Background: A loss of neostriatal neurons is a main characteristic of Huntington's disease (HD). Neuroplantation has been proposed as one of the potential treatments. Neuronal stem cells (NSCs) are primordial, uncommitted cells postulated to migrate and differentiate in a temporally and regionally appropriate manner.

We attempted to test the ability of NSCs migration into the lesion site in a rat model of HD.

Methods: Clonal human NSCs were used, which mirrored strategies previously described (Flax et al., 1998). To provide identification, cells were infected with retroviral vector encoding lacZ and neo for selection. Following quinolinic acid lesioning in unilateral striatum, NSCs were injected in the contralateral ventricle. In some rats, intravenous injection of NSCs was performed through the femoral or tail veins. Beta-galactosidase histochemistry was performed.

Results: NSCs migrated and were identified mostly in the lesioning site. These cells were distributed in clusters and some in the perivascular area, choroid plexus, or subependymal zone. The majority of cells appeared to be differentiated into GFAP positive cells.

Conclusion: NSCs migrate into striatum in HD model, either from nervous system or from systemic circulation. The developnent of a strategy for the stem cell application and functional recovery remains to be tested. 
Ih blocker has a potential for anti-epileptic effects

M. Kitayama (Toronto, Ontario), S. Kogure, H. Miyata, N. Saito, M.Y ano, H. Iwai, K. Yamamoto (Hachioji, Tokyo), Y. Matsuda, T. Yamauchi (Iruma, Saitama)

Background: The h current (Ih) is an inwardly-mixed cationic conductance activated by membrane hyperpolarization. We hypothesized that Ih channels might contribute to hippocampal epileptiform discharges, and have reported about the effects of Ih blockers on the electrically-induced paroxysmal discharges in vivo studies. Here, we summarized our results obtained from the acute experiments.

Methods: We used 60 adult male rabbits. We implanted the electrodes into the dorsal hippocampal CAl region. We stimulated and measured the threshold to induce epileptiform discharges (defined as PAD threshold). After the recovery period, we injected each agent ( $\mathrm{CsCl}, \mathrm{CsBr}, \mathrm{ZD} 7288$ and saline) into the apical dendritic layer. We examined alterations of PAD threshold before and after injection.

Results: We found that Ih blockers raised PAD threshold. On the other hand, we didn't observe the increase of PAD threshold in the saline injection group. The increase of PAD threshold in Ih blocker injection groups was more significant than that in saline injection group $(\mathrm{p}<0.05)$.

Conclusions: We concluded that Ih channels might contribute to hippocampal epileptiform discharges because lh blocker injection into the apical dendritic layer raised PAD threshold. Although preliminary, our data suggest that Ih blockers may be useful as antiepileptic drugs.

\section{P-047}

\section{Regenerating sensory axons have enhanced CGRP expression}

\section{Li, D.W. Zochodne (Calgary,Alberta)}

Background: Calcitonin gene-related peptide (CGRP), an alternative splice product of the calcitonin gene, is expressed in neurons, is mitogenic to Schwann cells, promotes nociception and increases nerve blood flow.

Methods: We examined CGRP expression using immunohistochemistry in L3- L5 dorsal root ganglia supplying the sural sensory nerve of the rat and local CGRP expression in regenerating sural fibers serially following crush.

Results: Following sural crush, an overall decline in the proportion of sensory neurons with CGRP expression was not apparent but there was a rise in the proportion of a subset of neurons with intense CGRP expression ipsilateral to the sural injury. This was associated with an increase in the numbers of CGRP expressing axons at and distal to the crush zone in both parent and branching axons. Interestingly, individual axons had enhanced CGRP expression confined to their distal, regenerating zones.

Conclusions: Enhanced local CGRP expression at sites of sensory axon injury, associated with a subset of intensely expressing CGRP sensory neurons in the ganglia, may play important roles in the regenerative milieu. These may include influences on the generation of neuropathic pain and on local Schwann cell proliferation.
Glutamate promotes proliferation of striatal neuronal progenitors by an NMDA receptor-mediated mechanism: An in vivo and in vitro study

\section{K.C. Luk, T. Kennedy, A.F. Sadikot, (Montreal, Quebec)}

Recent evidence suggests that neurotransmitters play an important role in the development of the mammalian central nervous system. We used in vivo and in vitro models to identify a novel role for glutamate in striatal neurogenesis mediated by N-Methyl DAspartate (NMDA) receptors. Quantitative analysis using unbiased stereology demonstrated that embryos exposed to NMDA receptor antagonists during striatal neurogenesis have a dramatic reduction in the total number of striatal neurons. Embryonic striatal neuroblasts exposed to NMDA antagonists in culture showed marked reduction in DNA synthesis, as measured by 5'-bromodeoxyuridine (BrdU) uptake. Low concentrations of NMDA increased proliferation significantly, whereas high concentrations were toxic. AMPA/KA receptor antagonists had no significant effect on striatal neuroblast proliferation either in vivo or in vitro. These results support the hypothesis that glutamate promotes proliferation of ventral telencephalon-derived striatal neuronal progenitors by an NMDA receptor-mediated mechanism. In contrast, previous findings suggest that proliferation of cortical progenitors derived from the dorsal telencephalon is regulated by activation of AMPA/KA but not NMDA receptors. This heterogeneous response to glutamate in the proliferative ventricular epithelium of the telencephalon may have important implications for pathogenesis of a wide variety of neurological diseases, including fetal alcohol syndrome, cerebral palsy and forebrain dysplasias.

\section{P-049}

\section{Multi-component $\mathrm{T} 2$ relaxation as a quantitative measure of} demyelination in vitro and in vivo

\section{R. Midha, G.J.Stanisz, S. Webb, C.A. Munro (Toronto, Ontario)}

Introduction: Changes in nerve tissue microstructure following nerve injury affect the Magnetic Resonance (MR) properties. Our goal was to evaluate the ability of multi-component $\mathrm{T} 2$ relaxation to quantitatively assess nerve damage.

Methodology: In Lewis rats, the right sciatic nerve was crushed; the left was cut and sutured to prevent regeneration. Nerves were imaged in vivo using a $1.5 \mathrm{~T}$ GE Signa whole-body scanner with scan sequence parameters optimized for $\mathrm{T} 2$-iveighted imaging. $\mathrm{T} 2$ data were fitted to a multi-component $\mathrm{T} 2$ model in which the relaxation of each T2-component has a Gaussian distribution on a logarithmic time-scale. Data were combined to create a T2-spectrum for the region of interest $(\mathrm{ROI})$.

At 1-6 weeks post-injury, nerve samples proximal and distal to injury were collected for in vitro MR and histopathologic analysis. MR measurements were performed $1.5 \mathrm{~T}$ on a $20 \mathrm{~cm}$ bore superconducting magnet. T2 was measured using a CPMG sequence.

Results: In vitro T2-spectrum of nerve shows three welldistinguished T2-components, hypothesized to relate to water found mainly in: myelin (short T2-component), intracellular space (intermediate T2) and extracellular space (long T2). At 1-2 weeks after injury, the size of the short T2-component is decreased. At 3-4 weeks, the short T2-component size for cut nerves remains reduced, 
whereas that of crushed nerve has increased, approaching normal values. At six weeks, T2 spectra and the histology of the crushed nerve resemble normal nerve. The short T2-component size correlates well with the morphometric myelin content for all time points. On in vivo $\mathrm{T} 2$-weighted images, nerve appears bright, easily distinguished from surrounding muscle and fat tissues. T2 spectrum for the ROI containing the sciatic nerve resembles the in vitro spectrum.

Conclusions: Changes in multi-component T2-spectra of injured nerves correlate well with changes in tissue microstructure. The size of the short T2 component reflects the amount of myelin present. T2weighted MR imaging shows great promise for quantitative evaluation of nerve damage.

\section{P-050}

Rescue of developing striatal neurons from apoptosis: role of thalamostriatal afferents

S. Mittal, M.A. Alonso-Vanegas, R. Aloyz, F.D. Miller, A.F. Sadikot, (Montreal, Quebec)

Background: The developing rat striatum receives massive input from the thalamus and cortex. We hypothesize that glutamatergic afferents to the developing striatum modulate apoptosis of specific neuronal types. We have previously shown that early thalamostriatal (TS) deafferentation results in massive apoptosis of striatal projection neurons, suggesting a trophic role for TS inputs.

Methods: We now examine whether different interneuron subtypes receiving input from either the thalamus or cortex show differential survival following postnatal TS lesions. Whereas cholinergic interneurons receive robust input from the thalamus, but only sparse input from the cortex, parvalbumin-positive (PV-ir) subsets are afferented predominantly by the cortex.

Results: Analysis using unbiased stereology shows significant loss of cholinergic and PV-ir interneurons in the adult striatum following lesions of the parafascicular $(\mathrm{Pf})$ thalamic nucleus at postnatal day 2 . These findings are in keeping with the hypothesis that glutamatergic TS afferents rescue striatal neurons from cell death.

Conclusions: The thalamostriatal system plays an important role in rescue from apoptosis of both projection and interneuron subtypes. We are determining the mechanism of this trophic effect, and extending the observations to other interneuron subpopulations of the striatum.

\section{P-051}

Comparing $\mathbf{N}$-acetyl-aspartate concentration and myelin water fraction in white matter

\section{R. Moll, A. Mackay (Vancouver, British Columbia)}

Background: T2 relaxation techniques for MR can be used to measure myelin water fraction (MWF) in vivo and MR spectroscopy can be used to measure relative concentrations of metabolites such as $\mathrm{N}$-acetyl-aspartate (NAA) and creatine (Cre). The current study investigates whether NAA/Cre ratios vary throughout brain white matter structures in a similar manner to MWF. The results should provide some insight into the behaviour of NAA throughout the brain, its function and whether MWF is proportional to volume of neuronal axoplasm.

Methods: Ten voxels were collected from seven healthy volunteers (two men, five women) who underwent MRS and MRI exams. The single voxel spectroscopy sequence placed voxels in frontal white matter $(n=5)$ and in the posterior internal capsules $(\mathrm{n}=5)$. Imaging was completed using a 48 echo CPMG pulse sequence. NAA/Cre ratios were calculated from spectra using LCModel.

Results: Myelin water fractions were lower $(0.02-0.12)$ than previous studies where white matter values were typically $0.05-0.15$. NAA/Cre ratios were in the range of literature values $(\sim 2.5)$ and had low standard deviations $(4-8 \%)$ The NAA/Cre ratio was plotted against the MWF. No significant trend between the two measurements was observed. There was a narrow spread of NAA/Cre (2-3) values.

Conclusions: It appears that NAA/Cre is fairly similar in frontal white matter and the posterior internal capsules. Thus there appears to be no correlation between amount of myelin water and neuronal axonal volume for these two brain structures.

\section{P-052}

Group 1 metabotropic glutamate receptors in neurotrauma: additive role of $\mathrm{mGluR1}$ and $\mathrm{mGluR5}$ in calcium-mediated axonal dysfunction

\section{N. Phan, N. Persaud, M. Zhao, E. Liu, A. Baker, M. Fehlings (Toronto, Ontario)}

Introduction: Following $\mathrm{SCI}$ and $\mathrm{TBI}$, secondary injury mechanisms promote axonal damage. We hypothesized that the mGluR 1 and mGluR5 receptors are involved in these processes and sought to determine their role in post-traumatic axonal dysfunction.

Methods: Western immunoblotting and immunohistochemistry were performed on rat corpus callosum following fluid percussion TBI. Compound action potentials (CAPs) were recorded in dorsal columns after $\mathrm{SCI}$ and corpus callosum brain slices following TBI. $[\mathrm{Ca} 2+]$ i fluorescence was measured in spinal cord slices in wild-type and mGluR1-/- mice.

Results: Immunoblots showed increased BAPP at 24hr, calpain activation at 30 minutes and $24 \mathrm{hr}$, and NF200 breakdown at $7 \mathrm{~d}$ following TBI. Axonal electrophysiological recovery following in vitro SCI and TBI improved with Group 1 blockade using PHCCC. $[\mathrm{Ca} 2+] \mathrm{i}$ imaging showed a reduction in post-traumatic $[\mathrm{Ca} 2+] \mathrm{i}$ rises with PHCCC and mGluR 1 gene deletion. Following TBI, Group 1 mGluRs blockade led to a CAP recovery of $77.1 \pm 10.2 \%$ compared to $37.6 \pm 5.4 \%$ in controls. mGluR I blockade led to a recovery of $52.9 \pm 7.4 \%$, while mGluR5 blockade resulted in $63.2 \pm 10.3 \%$ recovery. All results were statistically significant $(\mathrm{p}<0.01)$.

Conclusions: Our results suggest that Group 1 mGluRs are involved in axonal dysfunction following neurotrauma. Combined mGlurl and mGlur5 blockade leads to a better axonal recovery than blockade of each receptor alone.

\section{P-053}

Electrophysiological and histological correlation of diffuse axonal injury following fluid percussion traumatic brain injury in the rat

\section{N. Phan, E. Liu, M. Zhao, M. Fehlings, A. Baker (Toronto, Ontario)}

Introduction: Diffuse axonal injury (DAI) is associated with poor outcome following traumatic brain injury (TBI). In this study, we 
sought to determine and quantify the functional deterioration of axons following TBI and correlate this with histological markers of injury.

Methods: Adult male rats underwent fluid percussion-induced TBI. Compound action potentials (CAPs) were recorded in the corpus callosum at $3 \mathrm{~h}, 1 \mathrm{~d}, 3 \mathrm{~d}, 7 \mathrm{~d}$, and $14 \mathrm{~d}$ following injury and compared to shams ( $n=5 /$ group). Brains were harvested in shams, $3 d, 7 d$ and $14 d$ ( $n=4 /$ group), sectioned, and stained for APP and injured myelin using immunohistochemistry.

Results: Corpus callosum axonal conduction was significantly reduced at $3 \mathrm{~h}(48.8 \pm 3.3 \%), 1 \mathrm{~d}(54.9 \pm 3.1 \%)$ and $3 \mathrm{~d}(52.9 \pm 3.7 \%)$ compared to shams $(100 \%)$. CAP recovered partially at $7 \mathrm{~d}$ $(85.1 \pm 4.9 \%)$, but deteriorated by $14 \mathrm{~d}(53.2 \pm 7.8 \%)$. APP expression was significantly increased at $3 \mathrm{~d}$ (count:1966.3 \pm 232.0 ), $7 \mathrm{~d}$ (3052.5 \pm 265.1$)$, and $14 \mathrm{~d}(1156 \pm 218.8)$ compared to shams $(61 \pm 13.9)$. Injured myelin was increased at $3 d$ (count:664.3 \pm 194.1 ), $7 \mathrm{~d}(721 \pm 87.8)$, and $14 \mathrm{~d}(687.8 \pm 31.4)$ compared to shams $(52.3 \pm 4.4)$. APP and injured myelin deposition tended to be distributed caudally and laterally.

Conclusions: This study is the first to report an evaluation of the degree and temporal pattern of axonal dysfunction following TBI. Reversability was seen between $3 \mathrm{~d}$ and $7 \mathrm{~d}$ electrophysiologically but not histologically. This highlights the value and importance of combined functional and morphological approaches in the evaluation of experimental TBI, especially DAI.

\section{P-054}

\section{Rapamycin prevents experimental autoimmune neuritis in Lewis rats}

\section{J.Spies, T. Lin (Sydney, Australia)}

Background: Chronic inflammatory demyelinating polyradiculoneuropathy (CIDP) responds to immunomodulatory therapy but long-term immunosuppressive therapy is often required, with significant associated toxicity. Rapamycin is a relatively new immunosuppressive agent effective in preventing rejection after organ transplantation. Rapamycin has a more favorable side effect profile than cyclosporin, and does not cause nephrotoxicity. Experimental autoimmune neuritis (EAN) is an animal model for inflammatory demyelinating neuropathy.

Methods: We induced EAN in Lewis rats and administered rapamycin or placebo by daily intraperitoneal injection commencing just prior to the expected onset of paralysis (prevention group) or at the first signs of EAN (treatment group). Clinical grade and weight were recorded daily, neurophysiology and histological examination were performed at predetermined intervals.

Results: Rapamycin given prior to the onset of disease prevented the development of significant paralysis (mean clinical grade $0.9 \mathrm{vs}$ 3.8 in placebo animals; $p<0.0001$ ). Weight loss and neurophysiological abnormalities were significantly less in treated animals. Data is currently being collected on animals treated after the onset of clinical paralysis.

Conclusion: Rapamycin prevents the development of EAN in Lewis rats. If similar results are evident in animals treated after the onset of clinical disease, rapamycin may be an effective treatment for refractory CIDP.

\section{P-055}

\section{Normal appearing brain tissue MTR histograms in patients} with clinically isolated syndromes and early RRMS

\section{A. Traboulsee, (Vancouver; British Columbia); D.H. Miller \\ (London, UK)}

Background: Magnetization transfer ratio (MTR) histograms provide a global measure of disease burden in multiple sclerosis (MS). Previous studies have shown evidence of MTR abnormalities in normal appearing brain tissue (NABT) in MS patients with wellestablished disease. However, the earliest development of these MTR changes is not fully defined. We investigated the MTR histograms for NABT in clinically isolated syndrome (CIS) patients and in early RRMS patients.

Methods: MT imaging was performed in $40 \mathrm{CIS}$ patients, 28 early RRMS and 51 controls. Images were segmented using SPM99. MTR histograms were obtained for NABT, grey (NAGM) and white (NAWM) after extracting lesion masks. Brain parenchymal (BPF) was used as a marker for atrophy.

Results: NABT, NAGM and NAWM mean MTR were significantly reduced in CIS and early RRMS patients. NABT mean MTR was significantly decreased in CIS patients without T2W lesions. Whole brain atrophy was only seen in early RRMS.

Conclusion: Subtle NABT abnormality is detected in patients with CIS before the diagnosis of MS and are more pronounced in patients with early RRMS. Abnormalities are seen both in NAWM and NAGM. Patients without T2W lesions also have abnormal NABT MTR despite being at a lower risk of developing MS and disability.

\section{P-056}

Unchanging distracter elicits inhibitory effects on saccadic eye movements

C.A. Welte-Santana, L.G. Gawryszewski, C.H. Andrade-Moraes, L. Klausner-de-Oliveira, T. da S. Portugal, F.M. Ferreira (Niteroi. Brazil)

Background: Fixation point (FP) offset before visual target presentation (gap condition) decreases saccadic reaction time (SRT) in comparison to a condition in which FP remains visible (overlap condition). Our objectives were to test if a bilateral distracter (BD-20 deg eccentricity) changed the SRT to a target located at 10 degrees (Experiment 1) and to test if this effect was due to the ipsilateral (ID) or the contralateral (CD) distracter (Experiment 2).

Methods: SRTs to peripheral visual targets were recorded using a Photoelectric Eye Tracker.

Experiment 1: Overlap and gap conditions with (or without) BD were randomly mixed in each block ( 80 trials).

Experiment 2: Using just gap condition, there were blocks with constant distracter characteristic (BD, ID, CD or no distracter).

Results: Experiment 1: In the overlap condition, the BD did not affect SRT (186 ms vs. I84ms). However, in the gap condition, SRT was longer in trials with BD $(128 \mathrm{~ms})$ than without it $(120 \mathrm{~ms})$.

Experiment 2: No distracter SRT $(98 \mathrm{~ms})$ did not differ from CD SRT $(99 \mathrm{~ms})$ but both were shorter than ID SRT $(112 \mathrm{~ms})$ and BD SRT ( $107 \mathrm{~ms})$.

Conclusions: Unchanging distracter can elicit a SRT increasing similar to inhibition of return. 


\section{NeURomuscular}

\section{Insulin facilitates peripheral nerve regeneration}

\section{Q.Xu, D.W. Zochodne (Calgary, Alberta)}

Background: A potential action of insulin in facilitating peripheral nerve regeneration has not been examined using robust indices.

Methods: Swiss mice underwent crush or transection injury of the left sciatic nerve at the sciatic notch, then received insulin or saline (insulin $0.02 \mathrm{IU}$, ip, twice daily). Regeneration was addressed by measuring myelinated fiber number and maturation distal to crush at two weeks and by serially measuring, following sciatic transection, the rate of recovery of the interosseous $M$ potential innervated by sciatic-tibial motor fibers. Expression of insulin receptors through the sciatic nerve crush zone one week after injury was studied using polyclonal and monoclonal immunohistochemistry.

Results: Insulin was associated with enhanced maturation of regenerating myelinated fibers as indicated by larger axon and fiber diameters $14 \mathrm{~d}$ following crush. Recovery of the interosseous $M$ potential after transection was more rapid in insulin treated mice. There was enhanced local expression of insulin receptors in regenerating fibers distal to the crush zone.

Conclusions: Insulin facilitates peripheral nerve regeneration by acting through locally expressed insulin receptors. These receptors appear to undergo upregulated expression in regenerating fibers.

\section{P-058}

\section{In vitro study of hippocampal EEG rhythms}

\section{Zhang, C.P. Wu, T. Wong, W.P. Luk (Toronto, Ontario)}

The hippocampal EEG is generated via interaction of extrahippocampal inputs and intra-hippocampal network activities. However, it is not clear whether the hippocampus in isolation is capable of exhibiting population synchrony and spontaneous rhythms

To address this, we developed a novel preparation by isolating hippocampus or interconnected hippocampi from young or mature mice and placing the isolated hippocampal tissue in a perfusion chamber for electrophysiological recordings.

The isolated hippocampus displayed spontaneous rhythms of 1-4 $\mathrm{hz}$ that propagated along the ventro-dorsal axis. We provided convergent evidence suggesting that the rhythms represent GABAA IPSPs from a population of pyramidal neurons, resulting from synchronized discharges of GABAergic inhibitory interneurons. Muscarinic stimulation inhibited the slow rhythms and produced fast rhythms of 6-15 Hz and epileptiform responses. Similar slow rhythms were also observed in the hippocampal tissues isolated from young rats and gerbils

We suggest that the rodent hippocampus is able to sustain the slow rhythms when isolated in a relatively intact form from extrahippocampal structures. The spontaneous rhythms we observed are inhibitory in nature and their generation requires the network synchrony of GABAergic inhibitory interneurons. We are currently exploring whether the isolated hippocampus mimics other types of hippocampal EEG rhythms.

\section{P-059}

\section{Inclusion body myositis presenting with loss of plantar flexion}

H. Block, C. Robinson, C. Toth, J. Donat (Saskatoon,

Saskatchewan)

Background: Inclusion body myositis (IBM) is an idiopathic, progressive myopathy of an inflammatory, or possibly degenerative, nature. The typical weakness in IBM is a particular pattern affecting finger flexors and quadriceps.

Methods: We report on two patients with gastrocnemius weakness leading to loss of plantar flexion and a flat-footed gait.

Results: In these two patients, gastrocnemius biopsy revealed a pathological diagnosis of IBM based upon the presence of myopathic changes, muscle inflammation, rimmed vacuoles, and ubiquitin positive granular inclusions identified in vacuoles.

Conclusion: IBM can present with unique patterns of weakness, such as loss of plantar flexion and a flat-footed gait.

\section{P-060}

Myopathy with tubular aggregates - a case with hyperkalemia

J.Y.Chu, J. Bilbao (Toronto, Ontario)

Background: Tubular aggregates are tightly packed tubules derived from dilated terminal cisternae of the sarcoplasmic reticulum. They could be found on muscle biopsy in a variety of neuromuscular disorders including the potassium-related periodic paralysis.

Methods: Case report.

Results: A 42-year-old man presented with subacute onset of generalized muscle pain, cramps and weakness. Examination showed moderate upper limb distal weakness and mild lower limb proximal weakness. There was no inducible myotonia. His deep tendon reflexes were depressed throughout and the sensory examination was normal. His initial CPK was $1,298 \mathrm{U} / \mathrm{L}$ and peaked at $2,200 \mathrm{U} / \mathrm{L}$ by day 2 . All other routine blood work were normal. His EMG and NCS showed mild myopathic changes. A right quadriceps muscle biopsy showed no evidence of inflammation. There were abundance of subsarcolemmal intracytoplasmic masses which were identified histochemically to be tubular aggregates and confirmed by electron microscopy. On subsequent follow-up, his serum potassium was found to be elevated at $5.7 \mathrm{MMOL} / \mathrm{L}$. He was successfully treated with hydrochlorothiazide $25 \mathrm{mg}$ OD with subsequent normalization of his serum potassium and CPK.

Conclusions: Tubular aggregates in skeletal muscle have distinctive histochemical features. Their functional significance has been postulated to represent an adaptive response to increased calcium influx. The clinical, electrophysiological and neuropathological findings of this case will be presented together with a comprehensive literature review.

\section{P-061}

\section{Presymptomatic genetic testing in ALS}

M.M.Mezei (Vancouver, British Columbia), P.M. Andersen (Umea, Sweden)

The 1993 discovery of mutations in the gene encoding CuZnsuperoxide dismutase (CuZn-SOD) in 14-23\% of diagnosed familial 
ALS (FALS) cases and 2-3\% of apparently sporadic cases of ALS (SALS) was a major breakthrough in the counseling and care of patients with such mutations. Since 1993, over 100 diseaseassociated mutations have been found in FALS and SALS cases worldwide. The pattern of inheritance for these mutations falls into three groups: mutations with dominant inheritance with very high if not complete penetrance, a group with dominant inheritance with reduced disease penetrance (not all gene carriers will develop ALS but may pass the mutated gene on to the next generation), and a group with recessive inheritance (the patient has inherited a disease gene from both unaffected parents). Genetic counseling of patients and unaffected blood-relatives are very different depending on which of these three groups the particular family mutation belongs to.

Since 1995 we have provided genetic counseling and presymptomatic testing of unaffected relatives of ALS patients with different $\mathrm{CuZn-SOD}$ mutations. There has been an unexpected large request for such service by both SALS and FALS patients and their relatives. Our experience and guidelines for presymptomatic genetic testing in FALS and SALS will be presented.

\section{P-062}

Progressive bulbar palsy with juvenile onset (Fazio Londe disease) - a case report

\section{N. Shah, B. Banwell, (Toronto, Ontario)}

Introduction: A case of progressive bulbar paralysis of childhood, a rare, pure motor bulbar neuronopathy is presented.

Clinical evaluation: A 16-year-old girl with congenital ptosis and developmental delay presented with drooling, nasal regurgitation of fluids, dysphagia for liquids and solids, dysphonia and weight loss (40 pounds) of three months duration. Examination revealed mild fixed bilateral ptosis, a full range of extraocular movements, restricted orbicularis oris and palatal movement, prominent tongue fasciculations, weakness of neck flexors (grade 4-/5) and absence of weakness or fasciculations in the limb muscles. Reflexes were brisk. Intravenous Tensilon failed to improve her dysphonia or ptosis. Feeding studies showed pooling of both liquids and solids with aspiration, leading to placement of a G-tube.

Laboratory Results: Serum CPK, TSH, lactate, and hexosaminidase A and B levels, DNA studies for oculopharyngeal muscular dystrophy and MRI studies of the brain, cervical cord, and neck were normal. Electromyography showed scattered polyphasic potentials with giant polyphasic units, normal nerve conduction and negative repetitive stimulation studies.

Discussion: Clinical and electrodiagnostic assessment are consistent with the diagnosis of juvenile progressive bulbar palsy.The absence of limb weakness excludes the diagnosis of sporadic amyotrophic lateral sclerosis (ALS) at this point. A review of juvenile progressive bulbar palsy will be presented.

\section{P-063}

\section{Painful burning feet and small fibre neuropathy: a prospective study in an Australian population}

\section{P.J.Spring, WX. Yan, F. Yang, J.M. Spies (Sydney, Australia)}

Background: Painful burning feet are a common clinical problem. In isolated small fibre neuropathy (SFN) routine nerve conduction studies (NCS) are often normal, making diagnosis difficult. SFN occurs in diabetes, but is most commonly idiopathic.

Methods: We prospectively evaluated subjects with distal pain, burning or paraesthesia for evidence of a sensory neuropathy. Patients with diabetes or another definite cause of neuropathy were excluded. Patients and controls underwent clinical assessment, NCS, quantitative sensory testing (QST-CASE IV), autonomic function testing, skin biopsy for epidermal nerve fibre density (ENFD), antineuronal and antisulfatide antibody testing and routine neuropathy blood tests.

Results: Fifty-six patients have been studied (age range 20-87, mean 56 years). Controls were aged $30-79$ years. The most frequent clinical features were: burning dysaesthesia $(77 \%)$, paraesthesia (71\%), hyperaesthesia (66\%), and reduced distal pain and/or temperature sensation (91\%). NCS showed evidence of neuropathy in $36 \%$, QST was abnormal in $79 \%$ and ENFD was reduced, compared to controls, in over $70 \%$. One patient was subsequently found to have small cell lung carcinoma and one tomaculous neuropathy.

Conclusion: These results are comparable to those described in other predominantly Caucasian populations. ENFD and QST are minimally invasive tests that greatly improve the accuracy of diagnosis of SFN.

\section{P-064}

Autosomal dominant hereditary sensory neuropathy with gastro-oesophageal reflux and cough: report of a family

PJ.Spring, GA. Nicholson, C. Kok, A. Ing. P. Cremer, J.M. Spies, J.D. Pollard (Sydney, Australia); M. Bassett, R. Tuck (Canberra, Australia); P. Kerlin, S. Bowler, J. Cameron (Brisbane, Australia)

Background: In this study we characterized a family with an autosomal dominant hereditary sensory neuropathy (HSN 1) with gastro-oesophageal reflux (GOR) and cough. Although HSN I with acral ulceration is commonly caused by mutations in the serine palmitoyl transferase (SPTLCI) gene on chromosome 9, there is clinical and genetic heterogeneity. Cough is a well-recognized manifestation of GOR, but in the few known GOR pedigrees, cough and neuropathy have not been reported.

Methods: Twenty-seven family members provided clinical information and blood for genetic analysis. Symptomatic individuals underwent neurological, gastrointestinal and respiratory investigations.

Resuls: Seven individuals had an adult onset of cough, reflux symptoms and distal sensory loss without ulcers. Cough could be triggered by strong odours and could lead to syncope. Nerve conduction studies, sural nerve and skin biopsies demonstrated a sensory axonal neuropathy. Autonomic and gastric emptying studies were normal. Audiometry revealed sensorineural hearing loss (4 of 10 subjects). Oesophageal $\mathrm{pH}$ monitoring demonstrated multiple temporally-associated reflux/cough episodes. SPTLCI DNA sequencing was normal. Linkage to hereditary neuropathy loci, CMT2A, 2B, 2D, 2E and 2F, was excluded.

Conclusion: This family may represent a new genetically distinct variant of HSN 1, particularly affecting the oesophagus, and possibly associated with sensorineural hearing loss. 
A unique ulnar neuropathy in the hand

\section{PJ. Sweeney, AJ. Wilbourn, E. Browne (Cleveland, USA)}

A 33-year-old women presented with right hand weakness of eight months duration, and hand wasting of one months duration. Pain, parethesias and prior hand/limb trauma were all denied. Her general health was unremarkable.

The neurological examination was normal except for profound weakness and wasting of the right first dorsal interosseous (FDI) muscle. There were neither sensory nor neurocutanous abnormalities.

Electrodiagnostic (EDX) examination revealed total denervation of the right FDI, with all other hand muscles examined, including adductor pollicis, second dorsal interosseous and hypothenar, being normal. These findings suggested a focal lesion of the deep right ulnar nerve affecting solely the motor branch to the FDI muscle.

At surgery, a ganglion cyst was markedly compressing the FDI at the point where the small terminal branches of the ulnar nerve arborized within it. The cyst was totally removed by tracing its stalk back into the wrist joint in the space between the scaphoid and capitate bones.

Ulnar neuropathies in the hand are uncommon and for such a lesion to involve just one hand muscle is extremely rare. The unique location of the cyst, compressing the surface of the FDI, explains the clinical and electrical findings.

\section{P-066}

\section{HAART in a patient with motor neuron disease}

\section{J.Turnbull, J. Martin (Hantilton, Ontario)}

Background: ALS in a patient with HIV infection was reported to resolve following highly active anti-retroviral treatment (HAART). A 48-year-old nurse, previously well, developed ALS. She had wasting and weakness of arms and legs, widespread fasciculations, characteristic EMG changes in arms, legs, and paraspinal muscles, as well as hyper-reflexia and spasticity, progressive over three years. She developed dysarthria and dysphagia.

Methods: Antibodies to HTLV I and 2, HIV, enteroviruses, and hepatitis were negative in blood. In July 2000 she began HAART (lamivudine, zidovudine, nelfinavir) on speculation.

Results: Within one week, she felt differently. Within three months there had been improvement in gait and arm strength. Her swallowing and speech normalised. Her grip strength doubled (from $11 \mathrm{~kg}$ in 1998 , to $21 \mathrm{~kg}$ in 2001 ). A repeat EMG showed only mild active denervation in the intrinsic foot muscles, without denervation in the face, upper extremities, or the trunk.

Conc/usions: Although previous antiviral therapies in ALS have been disappointing, this patient with sporadic ALS improved and more recently stabilized. HAART may be more effective than previous antiviral treatments in patients with occult retroviral infection. Conversely, the protease inhibitors (including nelfinavir) may have independent anti-apoptotic activity. Further trials are warranted.
Upregulation of antioxidative enzyme in sporadic inclusion body myositis

F. Yang, M. Wang, A. Wei (Sydney, Australia); B. Knoops (Louvainla-Neuve, Belgium); R. Ouvrier, J. Pollard (Sydney, Australia)

Background: Sporadic inclusion body myositis (s-lBM) is characterised pathologically by the presence of degenerating fibres with rimmed vacuoles containing deposits of amyloid and "Alzheimer-characteristic" proteins. Additionally, ragged-red fibres are common, suggesting that oxidative stress may be important in pathogenesis of the disease. Anti-oxidative enzyme (AOE) is a new member of the recently identified peroxiredoxin family, a group of antioxidant enzymes that are evolutionarily conserved in all phyla and thought to play an important protective role against oxidative stress.

Methods: To investigate the possible role of oxidative stress in the pathogenesis of S-IBM, immunostaining using antibody to AOE (AOE-ab) and in situ hybridisation with an AOE cRNA probe were carried out on muscle biopsies of eight patients with s-IBM and eight normal controls

Results: Positive staining of rimmed vacuoles in biopsies from patients with S-IBM was seen by immunohistochemistry with AOE$\mathrm{ab}$ and upregulation of AOE-mRNA was detected by in situ hybridisation. Ragged-red fibres also reacted strongly with AOE-ab. AOE could not be detected in control biopsies using either method.

Conclusion: Expression of AOE is upregulated in degenerating muscle fibres from patients with s-IBM, supporting the theory that oxidative stress may be involved in pathogenesis of the disease.

\section{EPILEPSY}

\section{P-068}

\section{Hippocampal sclerosis following nonconvulsive status} epilepticus

\section{L.M. Bateman, P.A. Garcia, K.D. Laxer, N.M. Barbaro (San Francisco, USA)}

Background: Hippocampal sclerosis is the most common pathologic finding in patients with refractory temporal lobe epilepsy. Animal models suggest that recurrent partial seizures can lead to its development, but such a relationship has not been consistently demonstrated in human epilepsy.

Methods: Report of two patients with non-convulsive status epilepticus and radiographic evolution of hippocampal sclerosis related to their illnesses.

Results: Patient 1 is a 58-year-old woman who presented with complex partial status epilepticus of left temporal origin in the setting of chlamydia encephalitis. Patient 2 is a 14-year-old boy who presented at age 11 with recurrent right temporal seizures and a nonspecific febrile illness. Both had acute MRI scans showing enlargement and increased signal of mesial temporal structures on the side of seizure origin. Follow-up scans revealed ipsilateral hippocampal volume loss and increased signal consistent with mesial temporal sclerosis. Patient 1 had her seizures controlled with phenytoin monotherapy, while Patient 2 developed medically refractory partial seizures. He underwent a right temporal lobectomy 
three years after his initial presentation with a pathologic diagnosis of mesial temporal sclerosis.

Conclusions: Non-convulsive status epilepticus can lead to the development of radiographically apparent hippocampal sclerosis and, in some cases, refractory partial seizures.

\section{P-069}

Properties of after-discharges from cortical electrical stimulation in focal epilepsies

\section{W.T. Blume, D.C. Jones (London, Ontario)}

Background: We revisit the value of after-discharge (AD) properties in assessing epileptogenesis by studying: 1) thresholds (this abstract), and 2) morphology.

Methods: Motor and visual function were assessed in 24 patients by $50 \mathrm{~Hz}$ biphasic usually monopolar stimulation with a pulse duration of $0.3 \mathrm{msec}, 50 \mathrm{~Hz}$, and 1-18 mAMP strengths.

Results: A broad range of stimulus strengths in mAMPs produced ADs among patients and within each patient. Although little mAMP overlap in producing/not producing ADs at a given point occurred, moderate overlap occurred among and within patients within each lobe or functional area. Lowest mAMP producing ADs did not vary significantly among regions although the median and mean $(2.5,3.3)$ for the mesial occipital region was lower than that for other nonfrontal-non-limbic surfaces $(3.5,5.1)(p=0.347)$. Motor frontal areas had only slightly lower median and mean values $(3.25,4.58)$ than frontal convexity $(5.5,6.4)$ and mesial frontal $(5.25,5.56)$ $(\mathrm{p}=0.534)$.

Conclusions: The significant variability in stimulus strength producing ADs indicate: 1) stimulus parameters need to be determined empirically 2) that inherent interlobar differences contribute weakly to AD thresholds which may depend more on individual epileptic tendency or lesion type.

\section{P-070}

Incidence, etiology, treatment and outcome of status epilepticus at the Ottawa Hospital in adults over a ten year period

\section{J. Carter, R. Bhojwani, C. Corman, A. Guberman, (Ottawa, Ontario)}

Background: Status epilepticus (SE) is a major medical and neurological emergency that requires immediate treatment to avoid significant morbidity and mortality. No published Canadian studies of SE are available to allow comparison with US and European series. Demographic and health-care factors unique to Canada make such a study worthwhile.

Methods: A ten year (1990-2000) retrospective chart review of patients with a documented diagnosis of generalized convulsive SE (GCSE) was undertaken at the Ottawa Hospital (TOH). The primary objective of the study was to examine the causes, treatment, and outcomes of GCSE. Secondary objectives were to analyze predictors of mortality in GCSE and to compare the treatment of GCSE at TOH with current published treatment algorithms.

Results: Of the 70 patients who met the inclusion criteria, $76 \%$ had a previous history of epilepsy and antiepileptic drug withdrawal was the most prominent cause of SE. Other common causes were metabolic, CNS tumour and alcohol withdrawal or intoxication. Thirty-seven percent responded to first-line therapy (i.e. diazepam or lorazepam and phenytoin). The duration of GCSE was between 30 minutes and two hours in $70.3 \%$ of the time. The mortality rate was $9.9 \%$. The combined etiologies of anoxia, metabolic, CNS infection and hemorrhage were correlated with mortality. Tumour and anoxia were found to be correlated on their own with mortality.

Conclusion: The results from this study showed some differences from published US and European studies such as causes of GCSE; shorter duration of GCSE and lower mortality rate.

\section{P-071}

\section{Piracetam in the treatment of cortical myoclonus epilepsy}

Karen M. Barlow, Mary B. Connolly, Kevin Farrell (Vancouver British Columbia)

Background: Cortical myoclonus often impedes activitics of daily living and can be difficult to control. Piracetam has been reported to be effective in cortical myoclonus but most studies have involved adults. We describe the use of of piracetam in patients with intractable cortical myoclonus.

Methods: This retrospective study involved review of hospital records of patients treated with piracetam at a children's hospital.

Results: Eleven patients (seven female) between 4.5 and 29 years of age (mean 15.4) were treated with piracetam. The underlying disorders included Unverricht-Lundborg myoclonus epilepsy (4), absence epilepsy with eyelid myoclonia (2), mitochondrial disease (1), remote symptomatic epilepsy (2), cryptogenic Lennox-Gastaut (1), and cryptogenic (1). The dose ranged from 3.3-24 g/day (mean 10.5). Six patients had a marked improvement in myoclonus and four had mild improvement. Weight gain occurred in two patients and problems with concentration in one. One patient died secondary to the underlying mitochondrial abnormality.

Conclusion: Piracetam may be helpful in the treatment of refractory cortical myoclonus and is associated with few sideeffects.

\section{P-072}

\section{Bilateral Rasmussen's encephalitis}

\section{Kevin Farrell, Kenneth Poskitt, Glenda Hendson (Vancouver,} British Columbia)

Background: Rasmussen's encephalitis (RE) is characterized by onset of epilepsy in childhood, progressive cognitive deterioration and progressive hemiparesis. Simple partial motor seizures are common and RE is the commonest cause of epilepsia partialis continua (EPC) in children. Neuroimaging demonstrates progressive unilateral brain atrophy.

Methods: We describe a child with clinical and radiologic evidence of bilateral disease.

Results: This previously healthy boy developed ictal vomiting at two years eight months and epilepsia partial continua of the right hand four weeks later. MRI at four years demonstrated increased signal in the right hippocampus and pathologic examination demonstrated lymphohistiocytic perivascular inflammatory infiltrate encroaching into vessel walls and inflammatory cells, microglial nodules and gliosis in brain parenchyma. Cerebral angiogram was normal. Mitochondrial enzyme activities in muscle, metabolic studies, connective tissue studies, and investigations for infection were normal. Six treatments with pulse methylprednisolone, IVIG 
and plasmapheresis at monthly intervals were not effective. Treatment with cyclophosphamide every four weeks was associated with improvement. His EPC and seizures worsened at five years of age and MRI at that time demonstrated increased signal in the left hippocampus and atrophy of the right hippocampus.

Conclusion: This patient demonstrates that Rasmussen's encephalitis may occasionally affect both cerebral hemispheres.

\section{P-073}

\section{Propagation patterns of occipital seizures recorded subdurally}

\section{M.A. Fava, W.T. Blume (London, Ontario)}

Background: Based on EEG-video documentation of pentylenetetrazole-induced seizures in epileptic patients, Ajmone Marsan and Ralston (1957) postulated that occipitally originating seizures could propagate either in a supra-Sylvian or infra-Sylvian direction, determined largely by origin above or below the calcarine fissure. We thus analysed occipital seizure propagation patterns. As clinical manifestations of occipitally originating seizures may reflect the propagated site, such documentation may have significant clinical implications.

Methods: We visually analysed subdurally recorded occipitally originating seizure propagation characteristics among 16 patients undergoing bilateral subdural electrode coverage of the occipital, temporal and parietal lobes.

Results: All seizures propagated. Propagation direction did not correlate with seizure origin within the occipital lobe (mesial vs. lateral; supra-calcarine vs. infra-calcarine). Seizures propagated to both supra-Sylvian and infra-Sylvian regions in 10 patients, to infraSylvian only (temporal lobe) in six patients: exclusively supraSylvian spread never occurred. Seizures spread bilaterally in 10 patients, ipsilaterally only six, and never only contralaterally. Seizures propagated within 5 seconds of origin in 12 patients and over 5 seconds in four.

Conclusions: Propagation to other lobes was a consistent feature of occipitally originating seizures. However, direction of propagation did not reflect the intra-occipital origin. Early propagation and contralateral propagation were common features.

\section{P-074}

Functional MRI and concurrent intracranial EEG in a sheep model of frontal lobe epilepsy: evidence of amygdala involvement

\section{P. Federico, H.I. Opdam, D.F. Abbott, J.S. Archer, R.M. Wellard,} G.C.A. Fabinyi, A. Syngeniotis, G.D. Jackson (Melbourne, Australia)

We describe a sheep model of penicillin-induced focal spike and seizure activity with concurrent EEG and functional MRI (fMRI).

Seizures were generated by injecting penicillin to the right prefrontal cortex of ten adult sheep. Animals then underwent videointracranial EEG monitoring for seizure characterization. Functional MRI under general anaesthesia was later performed at 1.5 and 3 Tesla by measuring blood oxygen level dependent (BOLD) weighted signal intensity at different time points during the evolution of seizures.

High quality intracranial EEG and fMR images were obtained routinely. Focal spiking and seizures were seen in all animals. An average of $13 \pm 4.8$ seizures were seen per animal, lasting $27.3 \pm$
12.3 seconds. High quality functional MR images were obtained routinely. Regional BOLD signal intensity changes were observed during seizures at the seizure focus and ipsilateral amygdala.

We have developed a model of partial epilepsy in which seizures can be reliably elicited with concurrent fMRI and intracranial EEG. During unilateral seizures, focal BOLD signal changes occurred at the seizure focus and ipsilateral amygdala, suggesting the presence of a cortical-subcortical loop. This observation illustrates the potential of the model for understanding seizure generation, spread, and possibly the consequences of repeated seizures on the brain.

\section{P-075}

\section{Asystole during partial epileptic seizures: a case report and} review of the literature

\section{A. Gagnon, R. Desbiens, (Quebec City, Quebec)}

Background: Asystole due to partial epileptic seizures is a rare event reported in about 30 patients. Diagnosis follows documentation of asystole, syncope and EEG evidence of an antecedent seizure. Electrophysiologic changes consistent with global cerebral hypoperfusion during the clinical event should be present.

We report the case of a 28 -year-old man with clinical and electrophysiologic evidence of ictal asystole. A review of the literature is presented.

The patient is a 28-year-old right-handed man without relevant neurologic or cardiac past medical illnesses. On March 2001, he was admitted for a first nocturnal episode of secondary generalized seizure. He described déjà vu sensations. During a routine EEG, he began to complain of a strange unease with a rising epigastric sensation and sweating. Subsequently, tonic right gaze developed and he became unconscious for one minute with urinary incontinence. After regaining consciousness, he was slightly disorientated for some seconds. Simultaneously, left temporal epileptic discharges were seen on EEG followed by a 24 seconds asystole on ECG with high amplitude slowing followed by generalized suppression on EEG. AED therapy was started and a cardiac pacemaker was implanted. MRI was normal. At follow-up eight months later, the patient had not experienced any new auras or seizures.

Conclusion: Ictal asystole syndrome is a rare but potentially lifethreatening syndrome associated with partial epilepsy. When ictal symptoms are minimal or subtle, it may be misdiagnosed has a primary cardiogenic condition. Only ictal simultaneous ECG/EEG recording certify the diagnosis.

\section{P-076}

\section{Epilepsy surgery without specific lesions}

\section{G.R.Ganapathy, W.T. Blume, D. Munoz (London, Ontario)}

Background: Patients undergoing epilepsy surgery without demonstrable specific lesions have mediocre results. Are there indices distinguishing patients with good results from those with bad? We present an enlarged series compared to a 1997 abstract with longer follow-up.

Methods: Of 685 patients undergoing focal resection from 1989 to $1999,70(10 \%)$ had normal or non-specific histology. These were followed from $2-8.5$ years (mean 3.75 years). All 70 patients had 
normal neuroimaging and normal or non-specific histological abnormalities such as mild gliosis.

Results: 26 (37\%) of patients were seizure-free with or without medications and $29(41 \%)$ were unhelped. Standard temporal and other focal resections had similar outcomes. The following clinical indices had no influence on outcome: age at seizure onset, age at operation, duration of epilepsy; previous febrile seizures, trauma, infection; the presence of aura, "limbic" vs. non-limbic aurae, seizure frequency, or pre-operative status epilepticus. Analysing the first 10 pre-operative scalp EEGs revealed that only five of 20 patients with spike foci outside the operated lobe were seizure-free $(p<.0058) .11$ of 13 patients whose EEG showed no such spikes, generalised spike waves or multiple independent spike foci became seizure-free $\mathrm{p}=.0001$ ).

Conclusions: Scalp EEG may help determine which non-lesional patients will benefit from surgery.

\section{P.077}

Very fast $(250 \mathrm{~Hz})$ activity in association with the epileptic focus in a patient with temporal lobe epilepsy

\section{D.W. Gross, (Edmonton, Alberta); J. Gotman, (Montreal, Quebec)}

Background: The EEG is routinely recorded within a limited frequency range for both scalp EEG and intracranial or stereoencephalography (SEEG) recordings (typically between 0.3 $50 \mathrm{~Hz}$ ). Recent studies have suggested that clinically relevant information can occur outside this frequency range. We report a case in which very fast $(250 \mathrm{~Hz})$ oscillations were recorded at the epileptic focus in a patient with temporal lobe epilepsy (TLE).

Methods: Depth electrodes were implanted bilaterally in the temporal lobes using an image guidance system. During the SEEG investigation, the patient had a single prolonged SEEG recording with a sampling rate of $1000 \mathrm{~Hz}$, a high pass filter of $0.01 \mathrm{~Hz}$ and a low pass filter of $300 \mathrm{~Hz}$.

Results: The SEEG study demonstrated bitemporal interictal epileptic abnormalities with ictal onset confined to the left mesial temporal structures. Left temporal spikes had an electrical field involving the hippocampus and, to a lesser extent, the parahippocampal gyrus and surrounding structures. During the recording at $1000 \mathrm{~Hz}$, bursts of $250 \mathrm{~Hz}$ activity were observed immediately following left temporal spikes. These bursts were confined to a single electrode contact in the hippocampus.

Conclusions: We report the finding of very fast EEG oscillations at the epileptic focus in a patient with TLE, which occurred far outside the frequency range of conventional SEEG recordings. Since this activity correlated with the results of the conventional SEEG investigation, but had a more constrained electrical field, we believe that this information is clinically relevant and, therefore, further study is needed to determine whether routine investigation of the high frequency domain is warranted in clinical SEEG investigations.

\section{P-078}

\section{A family with autosomal dominant epilepsy and variable phenotype}

\section{D.W. Gross, P. Smyth (Edmonton, Alberta)}

Background: The etiology of as much as $40 \%$ of epilepsy is believed to have a genetic component. Of these, the vast majority of cases have a complex pattern of inheritance while relatively feıv are found to have a Mendelian pattern of inheritance. To date there have only been a small number of epilepsy syndromes described with an autosomal dominant pattern of inheritance including: familial nocturnal frontal lobe epilepsy, benign familial neonatal convulsions and generalized epilepsy with febrile seizures plus (GEFS+).

Methods: Detailed family history was obtained with clinical and EEG investigation of affected family members.

Results: Four affected family members were observed in four consecutive generations. Male to male transmission was observed confirming an autosomal dominant pattern of inheritance. One unaffected family member was observed to have an affected offspring. The seizure pattern for the affected family members in the first and second generation was afebrile generalized convulsions during childhood with complete spontaneous remission while the prepositus (third generation) has had a twenty-year history of medically intractable focal seizures suspected to be of frontal lobe origin. The affected person in the fourth generation has had a recent onset of generalized convulsions as an adolescent.

Conclusions: We report a family with an autosomal dominantly inherited epilepsy syndrome with variable phenotype, incomplete penetrance and some evidence of genetic anticipation. Although the variable phenotype of this family resembles the GEFS+ syndrome (which has been linked to mutations of sodium channel genes on chromosomes $19 q$ and $2 q$ ), the absence of febrile seizures suggests the possibility of a novel genetic defect.

\section{P-079}

\section{Functional hemispherectomy in children with Ohtahara} syndrome and hemimegalencephaly

\section{Hamiwka, I. Alfonso, E. Liu, M. Duchowny (Miami, USA)}

We report two cases of successful surgical treatment in children with malformations of cortical development presenting as early epileptic encephalopathy with suppression-burst (Ohtahara syndrome). Magnetic resonance imaging (MRl) showed cortical thickening and hemimegalencephaly. At the time of surgical evaluation a focal epileptic pattern was recorded on EEG. Scalp recorded ictal video-EEG revealed seizure onset from the abnormal hemisphere and ictal SPECT demonstrated hyperperfusion. Electrocorticography showed nearly continuous spike and slow wave discharges over the affected hemisphere. Taylor Type cortical dysplasia was observed on pathologic examination. Postoperatively both patients are seizure-free on monotherapy with a significant improvement in development. These findings emphasize the benefit of early surgical treatment for children with Ohtahara syndrome due to a unilateral malformation of cortical development.

\section{P-080}

\section{Carbamazepine-induced hypertension}

\section{N. Jette, T. Veregin, A. Guberman (Ottawa, Ontario)}

Background: The widespread use of carbamazepine in the management of epilepsy and other conditions is largely due to a favourable side effect profile. In general, cardiovascular side effects are uncommon with antiepileptic drugs, and are considered rare with carbamazepine. We were able to identify only a single report in the literature describing de novo hypertension in a patient started on 
carbamazepine. We report a second case of carbamazepine-induced de novo hypertension.

Merhods: A normotensive 33-year-old man with complex partial seizures was started on carbamazepine. He was followed for two years. Blood pressure was measured at each visits.

Results: Our patient developed hypertension at his first follow-up visit after starting carbamazepine. His hypertension persisted at follow-up visits. A workup for secondary hypertension and Cushing's disease was normal. Carbamazepine was discontinued and another antiepileptic agent was instated. His blood pressure normalized and remained normal over the next two years.

Conclusions: De novo hypertension should be recognized as a rare side effect of carbamazepine. Shared pharmacologic properties with the tricyclic agents may be responsible. Blood pressure should be monitored in patients started on carbamazepine, and carbamazepine therapy should be reassessed in all patients developing de novo hypertension while taking this drug.

\section{P-081}

Transient lesion in the splenium of the corpus callosum in an epileptic patient

S.M. Mirsattari, D.H. Lee, W.T. Blume (London, Ontario); M.W. Jones (Vancouver, British Columbia)

Background: Transient focal lesion of splenium of the corpus callosum (SCC) is rare in epileptic patients. Its pathogenesis is uncertain: frequent seizures, alteration of anticonvulsants (AEDs) causing ischemia or demyelination is possible. This is the only reported case with occipital epilepsy.

Methods: Several MRI sequences provided insight into pathogenesis.

Results: Following one week of telemetry monitoring of a 28year-old patient with occipital epilepsy, her carbamazepine was discontinued for five days. Five focal seizures with secondary generalization occurred four days after cabamazepine omission. 1.5T MRI shortly after seizures revealed a homogeneous $1 \mathrm{~cm}$ diameter circular lesion with distinct margins in SCC. It had increased signal abnormality on diffusion weighted, FLAIR, and T2weighted images and slightly reduced signal compared to corpus callosum on T1. It was not seen on 4T MRI images obtained two days prior to her seizures and completely resolved three months later. The absence of any identifiable signal on follow-up T2 or FLAIR images made infarct unlikely. Imaging features and ancillary tests excluded other causes. It likely represented focal ischemia from rapid reduction of AEDs, particularly carbamazepine. Subdural recordings did not show seizure propagation through SCC.

Conclusions: Rarely seen transient discrete focal lesions in SCC of patients with epilepsy is likely ischemic secondary to rapid alteration of AEDs.

\section{P-082}

\section{Simultaneous EEG and fMRI/MRS study of the occipital lobe} seizures

\section{S.M. Mirsattari, R. Bartha, F. Bihari, N. Rajakumar, L.S. Leung,} W.T. Blume, R.S. Menon (London, Ontario); J.R. Ives (Boston, USA)

Background: Functional MRI (fMRI) has the potential to overcome limitations inherent to EEG study of epileptiform activity and provide real time image guidance for metabolic measurements using magnetic resonance spectroscopy (MRS). The feasibility of simultaneous fMRI/MRS/EEG was investigated in an animal model of epilepsy.

Methods: Male Sprague-Dawley rats were anesthetized with ketamine/xylazine. Using a nylon stereotactic frame, carbon fiber electrodes were placed above the dura to continuously record EEG (Ives US Patent No: $5,445,162$ ). Epileptiform activity was induced in the visual cortex by intracortical injection of penicillin. Then EPI images (FOV $=5 \mathrm{~cm}$, 11 slices, $2 \mathrm{~mm}$ thickness, $64 \times 64$ matrix, TE/TR $=15 / 1700 \mathrm{~ms}$ ) were acquired using a $4 \mathrm{~T}$ Varian MR scanner followed by LASER localized MRS (TE/TR $=40 / 3200 \mathrm{~ms}, 125 \mathrm{ul}$ volume, 256 averages). Animals were then sacrificed and perfused for C-fos labeling.

Results: Activation maps were successfully constructed from fMRI data using a pixel cross-correlation method incorporating the low-pass filtered EEG as a reference vector. EEG was also successfully used to bin MRS data into 'active' and 'baseline' states. Metabolite levels such as glutamate, glutamine, $\mathrm{N}$-acetylaspartate, choline, and creatine were measured.

Conclusions: Preliminary measurements show the feasibility of obtaining simultaneous fMRI/MRS/EEG to study the neurobiology of different seizure states.

\section{P-083}

\section{Pseudodementia due to subtle seizures in cortical dysplasia}

\section{O. O'Mahony, M.B. Connolly (Vancouver, British Columbia)}

Background: Neurological regression is well-recognized with West syndrome and Lennox-Gastaut syndrome. It is less common in partial epilepsy. We describe a child with frontal lobe epilepsy who had neurological regression due to unrecognized seizures.

Methods: A description of the clinical course, investigations and evolution following frontal surgery.

Results: Seizures began at five years, characterized by a "pulling sensation" in the left arm, bilateral tonic stiffening, vocalization and speech arrest. They occurred nocturnally in clusters weekly. She did not respond to nine AEDs. At 10.5 years there was markedly deteriorated speech, attention, cognitive function and neuropsychological function since the first assessment at seven years. There was no obvious increase in seizure frequency. During video-EEG monitoring, 50 seizures of right frontal origin occurred nightly in sleep. MRI was normal. Ictal-SPECT demonstrated hyperperfusion in the right mesial frontal lobe. Resection of this area at 11 years resulted in marked improvement in seizures and cognitive function. Further right frontal resection was performed at 13 years and the patient has been seizure-free for 42 months since. Pathology demonstrated cortical dysplasia. Postoperative neuropsychological testing has improved compared to the preoperative analysis.

Conclusions: Seizures are an important treatable cause of deterioration in individuals with epilepsy and should be considered even with partial epilepsy.

\section{P-084}

Surgical resection for cortical dysplasia: correlation of seizure outcome with pathological changes in the resection margin

O. O'Mahony, G. Hendson, P. Steinbok, M.B. Connolly (Vancouver, British Columbia)

Background: Cortical dysplasia is a common cause of refractory epilepsy. Defining the extent of surgical resection may be difficult. 
We hypothesized that involvement of the resection margin in the pathological process would predict poor surgical outcome.

Methods: Children with medically refractory epilepsy were evaluated with invasive EEG monitoring using subdural electrodes. The area of resection was determined by ictal onset zone and functional mapping of eloquent cortex. The margins of the resection were analyzed for involvement in the pathological process.

Results: Ten children, aged 2-16 years at time of surgery had frontal resection $(n=5)$, parietal resection $(n=2)$ frontotemporal resection $(n=2)$ and temporal resection $(n=1)$. MRI was normal in eight and two demonstrated a subtle abnormality. Follow-up ranged from eight months to six years. Six patients are seizure free, two have had a worthwhile improvement (Engel IIIA) and two are unchanged (IVA). In 9 of 10 patients the pathological changes extended to the resection margin.

Conclusions: Seizure control following surgical resection for cortical dysplasia did not correlate with involvement of the resection margin in the pathological process.

\section{P-085}

\section{Latency of EEG abnormalities in prolonged EEG recordings}

\section{T. Ottavio, A. Mohamed (Sydney, Australia)}

Background: Prolonged EEG recordings are an important diagnostic tool. However, little is known about the necessary duration of testing and the sensitivity of prolonged recordings.

Methods: We retrospectively reviewed outpatient video EEGs performed at Royal Prince Alfred Hospital from I/98 - 1/99. The reason for referral, provisional diagnosis, and abnormalities in the final report were recorded. The latency to the first instance of abnormality noted in the final report was recorded.

Results: A total of 70 normal and 83 abnormal EEGs were reviewed. The majority of the patients with normal EEGs $(32 \mathrm{M}$, $38 \mathrm{~F}$ ) were referred from neurologists. $70 \%$ of patients with abnormal EEGs $(35 \mathrm{M}, 48 \mathrm{~F})$ were also referred from neurologists for investigation of suspected epilepsy or diagnosis of turns. The commonest abnormality was intermittent focal slowing (42\%) and sharp waves $(25 \%)$. The average the latency of abnormalities from the onset of recording included: intermittent focal slowing $(65$ minutes), focal sharp wave (65 minutes), generalized epileptiform activity (108 minutes), seizure (120 minutes) and pseudoseizures (103 minutes).

Conclusions: The duration of EEG recording should be tailored to the abnormality sought. A three-hour sleep recording will usually detect interictal epileptiform abnormalities. Whole day recordings may be necessary for capture of seizures or pseudoseizures.

\section{P-086}

\section{Anticonvulsants and cutaneous changes: a study of photographs of monozygotic quintuplets}

\section{G.M.Remillard, F. Remillard, A.L. Sherwin, E. Andermann,}

\section{F. Andermann, B.G. Zifkin (Montreal, Quebec)}

Background: The Dionne quintuplets were often photographed. One of us (GMR) noted a facial asymmetry in the one with childhood-onset epilepsy and studied their photographs further. From birth to age 18 years, the Dionne quintuplets lived and were educated together in a specially built home. They were the object of intense media attention. Emilie developed epilepsy and was treated before age eight years.

Methods: We studied all the illustrations in a 1995 book on the Dionne quintuplets, who were born in Canada in 1934. The three surviving quintuplets have been photographed into their sixties and many photographs show all five in their first 20 years. Emilie died at age 20 as a result of an epileptic seizure, and Marie, smallest and last born, died later.

Results: Photographs show that before the middle of her second decade Emilie developed subtle facial differences compared to her sisters, with thicker and wider eyebrows, a rounder contour of the cartilaginous part of her nose, and rounder cheeks and lips which we attribute to phenytoin. We could not see in the photographs whether or not she had gingival hypertrophy.

Conclusions: The progressive skin and subcutaneous changes seen in photographs of the single Dionne quintuplet with epilepsy may have been due to early and continued treatment with phenytoin

\section{P-087}

Frontiers in epilepsy surgery: initial lessons from deep brain stimulation

A.F. Sadikot, F. Andermann, D. Gross, F. Dubeau, A. Olivier

(Montreal, Quebec)

Background: Deep brain stimulation (DBS) is now a wellestablished procedure for surgical treatment of movement disorders. Recently, DBS has been proposed for reducing seizure tendency. DBS of the centromedian nucleus of the thalamus, the anterior thalamic area, and the subthalamic nucleus is encouraging in preliminary results.

Methods: We have operated on a limited number of patients, using the vagus nerve, thalamus, or hypothalamic hantartoma as targets for brain modulation in epilepsy. We report on the rationale, depth EEG findings and clinical course of two patients with DBS leads inserted at subcortical targets.

Results: A patient with severe generalized epilepsy was observed for two years following bilateral centromedian DBS. Reduction in seizure tendency was noted, and withdrawal of stimulation was associated with increased seizure frequency. A second patient presented with gelastic seizures associated with a hypothalamic hamartoma. DBS leads were inserted into the anterior thalamus and in the hypothalamic hamartoma. Reduced seizure tendency was noted on six month follow-up.

Conclusions: Besides vagal nerve stimulation, which has known but limited therapeutic benefit, DBS for epilepsy is an as yet unproven therapy. Careful experience with DBS in major centers will likely lead to emergence of different central targets indicated for specific seizure types.

\section{P-088}

Effects of Ih blockers, $\mathrm{CsCl}$ and $\mathrm{ZD7288}$, on inherited epilepsy of Mongolian gerbil

N. Saito, M. Taira, K. Yamamoto, H. Miyata, M. Yano, S. Kogure (Hachioji, Tokyo)

Background: In our acute experiments, $\mathrm{CsCl}$ and $\mathrm{ZD} 7288$, which were blockers for hyperpolarization-dependent cationic current (Ih), increased the threshold of electrically-induced paroxysmal 
discharge. We examined the anticonvulsant effects of Ih blockers on inherited epilepsy of Mongolian gerbil.

Methods: Seizure-sensitive gerbils $(\mathrm{n}=22)$ were employed. They were tested by the falling-stimuli once or twice a week for 5-6 weeks before, during, and after orally administering $\mathrm{CsCl}(10 \mathrm{mM})$ or ZD7288 $(100 \mu \mathrm{M})$ solution. The behavioral responses were classified into four stages. We measured the ratio of each response to total examination.

Results: In $\mathrm{CsCl}$ dosing test, the ratio of generalized seizure was $17 \pm 5.5 \%$ (mean \pm S.E.) during the control period $(n=122), 3.1 \pm 2.3 \%$ during the examination ( $n=109)$, and $7 \pm 7.0 \%(n=127)$ during the recovery. The ratio of no responses rose to $67 \pm 3.6 \%$ from $59 \pm 5.2 \%$ (control) and $52 \pm 8.0 \%$ (recovery). ZD7288 also reduced the ratio $(10 \pm 2.6 \%: n=88)$ of generalized seizure in control to $0 \%(n=71)$, while it increased the ratio $(24 \pm 2.5 \%)$ of no responses to $46 \pm 2.9 \%$.

Conclusions: Since statistical analyses showed a significant difference $(\mathrm{p}<0.01)$, it is concluded that $\mathrm{CsCl}$ and $\mathrm{ZD7288}$ have a potent anti-epileptic effect.

\section{P-089}

\section{SPECT scanning in musicogenic epilepsy}

K. Schoffer, RM. Sadler, D. Barnes, J. Matte (Halifax, Nova Scotia); R. Leckey (Sydney, Nova Scotia)

Background: Musicogenic epilepsy has been strongly correlated with right temporal lobe seizures. We present a 69-year-old woman whose musicogenic complex partial seizures began at age 15 years. Her clinical features were previously reported (Murray TJ, CMAJ 1984).

Methods: The patient underwent MRI, video-telemetry EEG, and ictal and interictal SPECT scanning with technetium $99 \mathrm{~m} \mathrm{ECD}$ injection.

Results: Interictal EEG showed left temporal spikes. An ictal EEG demonstrated left temporal ictal rhythms. MRI showed no structural abnormality in the temporal lobes. An interictal SPECT scan was unremarkable but ictal scanning demonstrated accumulation in the left temporal region.

Conclusions: To our knowledge, this is the first reported case of left temporal lobe originating musicogenic epilepsy which has been demonstrated on SPECT scanning.

\section{P-090}

\section{Hippocampal dysplasia in a patient with intractable seizures and partial trisomy 9}

\section{B.M. Wheatley, D.B. Sinclair, C. Hao (Edmonton, Alberta)}

Background: The clinical features of patients with trisomy 9 have been described. Included among the clinical features are dysmorphic facies, developmental delay, and mental retardation. Hippocampal dysplasia has not been described in patients with partial or complete trisomy 9.

Case report: A male was born at term to a 27 -year-old G2P0 mother. He experienced seizures immediately after birth requiring intubation. Over an 18-month period, the patient was admitted multiple times for recurrent status epilepticus. Clinical examination revealed low set ears, micrognathia, inguinal testes, small mouth, low hairline, a hypoplastic scrotum, saw tooth shaped teeth and scoliosis. Long-term video EEG monitoring demonstrated complex partial seizures originating independently from both temporal lobes.
An MRI showed widened subarachnoid space with widened perisylvian / opercular space and partial agenesis of the corpus callosum but no specific cortical abnormality. Genetic analysis identified an unbalanced translocation of chromosome 9 and 17 ( $t$ $(9 ; 17))$ resulting in partial trisomy of chromosome 9 . The patient eventually died after a respiratory infection and refractory status epilepticus. Pathological examination of the brain identified bilateral hippocampal dysplasia.

Conclusion: Patients with partial or complete trisomy 9 may have hippocampal dysplasia or malformations of cortical development as part of their symptom complex.

\section{P-091}

Epileptogenesis induced by alternate-site kindling of the rabbit hippocampi

M. Yano , H. Iwai, S. Kogure, (Hachioji, Tokyo); Y. Matsuda, T. Yamauchi, (Moroyama, Saitama)

Background: Recently, it has been reported that alternate-site kindling (AK) between two forebrain structures induces the phenomenon known as kindling antagonism (Teskey et al., Epilepsia, 1999). Based on our previous research (Kogure et al., Epilepsia, 2000), AK between both hippocampi was investigated to clarify whether AK would induce the kindling antagonism or not.

Methods: Ten adult male rabbits served as subjects. Their bilateral hippocampi were alternately stimulated once every $24 \mathrm{~h}$ according to Goddard's method (80-160 $\mu \mathrm{A}, 1 \mathrm{~ms}$ pulse, $50 \mathrm{~Hz}$, Isec application).

Results: All animals developed a stage 5 convulsion with a mean of 27 stimulations: It takes only 13 unilateral hippocampus stimulations to achieve complete kindling. Progressive alterations in behavioral stage induced by each hippocampal stimulation were not different. An averaged alteration of afterdischarge duration was a gradual increment in both sides. Simple A type of interictal discharge occurred at a higher rate during AK, while simple B type was lower level.

Conclusions: It was concluded that AK was appropriate model of hippocampal kindling of the rabbits. The reason why the kindling antagonism did not work in the present AK procedure is considered due to the decline in the level of feed-forward inhibition.

\section{General Neurology}

\section{P-092}

\section{Vestibular symptoms due to venlafaxine withdrawal: a drug} dependence syndrome

\section{Hersch, L. Allport (Sydney, Australia)}

Four eloquent professionals had difficulty describing intrusive, unpleasant symptoms of dysequilibrium, which occurred within 2436 hours of ceasing low-dose venlafaxine (Efexor), a widely used antidepressant that blocks re-uptake of serotonin and noradrenalin. Two of the patients experienced similar withdrawal symptoms if a single dose was omitted or delayed. In each patient, the symptoms were reproducibly and rapidly terminated by administration of venlafaxine. Physical examination revealed no signs of vestibular dysfunction in any patient. Although all four patients have recovered from depression, three remain physically dependent on venlafaxine despite repeated attempts to discontinue the drug. 
The pathogenetic mechanism of venlafaxine withdrawal is uncertain, but may involve down-regulation of serotonin and noradrenalin receptors subsequent to high local neurotransmitter concentrations following re-uptake blockade.

Venlafaxine discontinuation resulted in dysequilibrium suggestive of vestibular dysfunction in each of our patients, but in none was the link recognised at first. Doctors prescribing venlafaxine, and patients taking this useful antidepressant, should be aware that there is a significant risk of disabling dysequilibrium and frank physical dependence. Unrecognised, bizarre withdrawal symptoms, including dysequilibrium, may lead to unnecessary investigations or a misdiagnosis of psychosomatic disorder. Finally, the study of venlafaxine-induced vestibular dysfunction may contribute to furthering our understanding of vestibular physiology.

\section{P.093}

Positron emission tomography of the brain in a case of ethylene glycol intoxication

J.M. Boulanger, S. Gosselin, F. Bénard, T. Nawar, (Sherbrooke, Québec)

Background: lmaging studies of the brain showing variable degree of deep gray structures and brainstem involvement in ethylene glycol intoxication have been reported but to our knowledge, a metabolic study of the brain has never been described.

Objective: To report the findings of positron emission tomography (PET) in a patient with ethylene glycol intoxication.

Clinical presentation: A 56-year-old man presented with progressive vertigo, dysarthria, quadriparesis and coma over eight hours. He also had acute renal failure, metabolic acidosis with increased lactate. Magnetic resonance imaging (MRI) of the brain revealed symmetrical hyperintense lesions on T2-weighted images in basal ganglia, thalamus, midbrain and medial temporal lobes suggestive of a toxic disorder. Renal biopsy revealed tubular deposits of birefringent crystals. PET showed hypometabolism in left caudate nucleus, bilateral thalami, left putamen and left midbrain. The patient completely recuperated neurologic function over four days and renal function over three months. Brain MRI repeated after four weeks had normalized. The diagnosis of ethylene glycol intoxication was made on the basis of the clinical and laboratory features. The patient had drunk homemade wine a few hours before admission.

Conclusion: Basal ganglia, brainstem and thalamic hypometabolism can be demonstrated in ethylene glycol intoxication.

\section{P-094}

\section{Miller Fisher syndrome: a retrospective chart review}

\section{S.D. Brass, M. Veilleux, (Montreal, Quebec)}

Background: Miller Fisher syndrome (MFS) constitutes $5 \%$ of cases of Guillain-Barre syndrome and is characterized by the triad of ataxia, areflexia and ophthalmoplegia with minimal limb weakness.

Design/Methods: Charts of patients with diagnosis of MFS seen at the Montreal General Hospital between 1989 and 2000 were reviewed retrospectively.

Results: Eight patients were identified with a mean age of 43 years (range: 17-70) and a female:male ratio of 5:3. Respiratory tract infection preceded neurological symptoms in three cases and gastroenteritis in three others. The most common initial symptom was diplopia that was found in all eight cases. Areflexia, ophthalmoplegia and ataxia were present on initial examination in $50 \%, 100 \%$ and $100 \%$ of cases, respectively. Cerebrospinal fluid findings demonstrated an albuminocytological dissociation in three cases. Brain neuroimaging was normal in three patients who had cranial CT and in one who had MRI. Electrophysiological studies obtained in seven patients were abnormal in five and normal in two. Three patients were treated symptomatically, two with intravenous immunoglobulin and three with plasmapheresis. Follow-up available for five patients revealed complete recovery in one patient and mild residual deficit in four patients.

Conclusion: Diplopia and ophthalmoplegia were the initial presenting symptom and sign in all eight cases and outcome was usually benign.

\section{P-095}

\section{What does a neurology trainee do?}

\section{SA.Broadley, P.D. Thompson (Adelaide, Australia)}

Background: Few studies have been undertaken to define the caseload of neurologists in training. In order to plan training programmes and potential research projects, information concerning the spectrum of diagnoses in a particular clinic would be invaluable.

Methods: A simple database of patient details and their primary diagnoses seen by a single neurology trainee (SB) during one year was maintained. The nature of each consultation was recorded. The most up-to-date diagnosis for each patient was used for analysis. All patient contacts (in-patient admissions, ward referrals, out-patient clinics and EMG clinics) were recorded.

Results: During one year there were 758 clinical contacts with 631 patients. In addition 141 NCS/EMGs were performed and 238 EEGs were reported and reviewed. The commonest categories of disease were movement disorders (13\%), pain syndromes including headache (12\%), neuropathies (11\%) and epilepsy (10\%). In $13 \%$ of cases, no specific neurological diagnosis could be made.

Discussion: Clinical time commitments for neurology trainees are high ( 900 contacts per year) and ensure a broad exposure to a wide range of neurological conditions. Many common neurological conditions are seen in sufficient numbers to provide a substrate for clinical research.

\section{P-096}

Suffering, a clinical outcome measure in the treatment of classical migraine headache

\section{B.M. Clarke, A.R.M. Upton, C.M. Castellanos (Hamilton, Ontario)}

Background: Migraine treatments focus on control of pain but in other chronic disorders (arthritis and epilepsy) suffering (perceptions of threat to one's idea of self) and pain are separate but sometimes related phenomena and suffering is an important outcome measure. Suffering in migraine has not been investigated.

Methods: Twenty (20) migraine subjects (mean age 41.63 \pm 11.29 SD years; two males) were given a validated questionnaire in a pilot study to assess suffering (Likert-type scale from I to 5). All subjects were employed (mean length of employment $12.03 \pm 8.75$ SD years). Mean duration of headache was $26.47 \pm 28.4$ SD hours. 19/20 subjects reported $6.87 \pm 17.62 \mathrm{SD}$ lost work days/year. 
Results: Results are consistent with our study of 240 patients with other chronic disorders (arthritis and epilepsy). Pearson correlation coefficient between total pain and suffering scores are weak at $r=0.378, p=0.10$. Endorsement frequencies show that 35 $40 \%$ of subjects believe migraine headaches will negatively affect their relationship with children, aging, job performance and future life. Migraine subjects $(n=19 / 20)$ have more hopes for the future than epileptic or arthritic subjects.

Conclusions: Suffering and pain are separate but related phenomena impacting on migraine patients. Suffering is an important clinical outcome measure.

\section{P-097}

\section{Case of neurotrichinosis in northeast Quebec: first report of diffusion-weighted MRI}

\section{A. Gagnon, M. Thibault (Quebec City, Quebec)}

Background: Trichinosis is a parasitic disease which develops after ingestion of undercooked meat contaminated with Trichinella spiralis. Severe forms occur in 5-10\% of cases and include CNS dysfunction. Neurotrichinosis is a distinctive cardio-neurological syndrome characterized by: (I) early onset of neurological symptoms; (II) diffuse encephalopathy and focal neurologic deficits; (III) concomitant acute myocardial injury; (IV) marked hypereosinophilia ( $\left.24000 / \mathrm{mm}^{3}\right)$; (V) CT scan showing small lesions in the hemispheric white matter or cortex.

We report the case of a 49-year-old native woman with clinical, laboratory and radiological evidence of neurotrichinosis and described, to our knowledge, the first report of diffusion-weighted MRI.

This 49-year-old woman was admitted with a seven-day history of fatigue and severe myalgias. During the last 48 hours, she was confused with a left hemiparesis. Initial neurologic exam showed stupor, stiff neck, Kernig's sign, tetraparesia and bilateral Babinski sign. Blood cell count revealed marked eosinophilia $\left(9600 / \mathrm{mm}^{3}\right)$. CSF contained $263 \mathrm{mg} / \mathrm{dl}$ of protein, 3 eosinophils and increased lactate. Troponin was increased at $18,9(\mathrm{~N}<0,5)$ and ECG showed anterolateral ST-T segment depression. MRI demonstrated multiple bilateral $\mathrm{T} 2$ signal hyperintensity in the periventricular white matter and occipital cortex. Lesions appeared black on ADC map, consistent with described neuropathologic findings of small ischaemic cavities. A masseter muscle biopsy confirmed the presence of encysted larvae. Treatment included oral albendazole and dexamethasone. Neurological symptoms improved gradually.

Conclusion: Diffusion-weighted MRI adds to the hypothesis of diffuse distal ischemia in neurotrichinosis. In Amerindian, differential diagnosis of acute encephalopathy should include neurotrichinosis.

\section{P-098}

\section{Adult onset of neurological symptoms in Sturge-Weber syndrome}

\section{M.S. Hussain, Z. Rubin, W. Johnston, D. Emery, J. Lewis}

(Edmonton, Alberta)

Background: Sturge-Weber syndrome is a neurocutaneous syndrome involving port-wine stains in the ophthalmic branch of the trigeminal nerve (VI) distribution and CNS malformations.
Individuals with Sturge-Weber syndrome develop neurological symptoms in childhood, most commonly seizures. There are no cases reported in the literature of adult onset of neurological symptoms in Sturge-Weber syndrome.

Methods: Case report/literature review

Results: A 45-year-old man presented with visual changes including a new left homonymous hemianopsia and generalized tonic-clonic seizures. The patient had no significant past medical history other than a port-wine stain over the majority of his face, including the right VI distribution. A head CT showed choroid plexus enlargement in the right occipital horn and right occipital cortical atrophy. MRI showed mild focal encephalomalacia in the right occipital lobe, overlying leptomeningeal enhancement, and ipsilateral choroid plexus enlargement. EEG revealed generalized slowing. At three month follow-up, the patient's left homonymous hemianopsia had resolved.

Conclusion: This patient presented in adulthood with CNS symptoms consistent with Sturge-Weber syndrome. As serious and treatable complications may develop at any time in the course of illness, it is important to monitor these patients beyond adolescence.

\section{P-099}

\section{Spinocerebellar degeneration in South African blacks}

\section{J. Joubert (Melbourne, Australia)}

Background: Spinocerebellar degeneration in African blacks has only rarely been recorded.

This study describes 50 consecutive black patients with spinocerebellar degeneration seen between 1983 and 1993.

Methods: All the patients were clinically examined by the author and had investigations including cerebral CT and in some cases MRI, biochemistry, viral studies, ophthalmological examination and evoked responses.

Results: There were 24 men and 26 women. The mean age was 33.3 yrs (range 14-62yrs). Autosomal dominant inheritance was present in five and recessive inheritance in one, while $88 \%$ were sporadic cases. The average duration of symptoms was 5.16 yrs. No patient had features of Friedreich's ataxia. Abnormalities of eye movement were documented in $36 \%$ and decreased visual acuity in $12 \%$. Retinal abnormalities were seen in $18 \%$. Extrapyramidal features were present in one case, $40 \%$ had hyperreflexia and $34 \%$ increased tone, I4\% had spastic gait and $10 \%$ were bedridden. Several had retinal degeneration some with amino-acid abnormalities in both serum and urine. All were HIV negative.

Conclusion: Spinocerebellar degeneration, mainly sporadic, is not uncommon in black Africans, in fact, being the commonest neurodegenerative condition seen in blacks at the Medical University of Southern Africa. It has a pleomorphic presentation.

\section{P-100}

Tuberous sclerosis associated with subependymal giant cell astrocytoma, bilateral renal angiomyolipomas, liver adenomas, cardiac rhabdomyoma, pulmonary lymphangioleiomyomatosis and pelvic endometrioma without mental retardation

\section{Kelly, V.Sadanand, R. Griebel (Saskatoon, Saskatchewan)}

Background: Tuberous sclerosis is a relatively common disease. We present a rare case of a patient without mental retardation but with 
multiple intra and extra CNS hamartomas secondary to tuberous sclerosis. This has not previously been described in the literature.

Methods: The patient was a 23-year-old female diagnosed with tuberous sclerosis at birth. She presented with adenoma sabeceum, infantile spasms without mental retardation, subependymal giant cell astrocytoma, multiple cortical tubers, bilateral renal angiomyolipomas, liver adenomas, cardiac rhabdomyoma and lymphangioleiomyomatosis and pelvic endometrioma. Her sister has tuberous sclerosis.

Results: The patient underwent magnetic resonance imaging of her brain. A small symptomatic subependymal giant cell astrocytoma was identified and was treated surgically with craniotomy. Multiple subependymal cortical tubers were observed. Computerized tomography, MRI and ultrasound scanning of the abdomen revealed bilateral angiomyolipomas, multiple liver adenomas and an endometrioma. Plain chest X-ray and CT scanning of the chest revealed a pneumothorax and lymphangioleiomyomatosis. A chest tube and pleurodesis were used in the management of the pneumothorax and lymphangioleiomyomatosis.

Conclusion: We present what we believe to be the first reported case of tuberous sclerosis associated with subependymal giant cell astrocytoma, bilateral renal angiomyolipomas, liver adenomas, cardiac rhabdomyoma, pelvic endometrioma and lymphangioleiomyomatosis without mental retardation.

\section{P-101}

\section{Bilateral tonic pupils in Vogt-Koyanagi-Harada syndrome}

J.S.Kim, C.H. Yoon, C.S. Moon (Cheju, Korea)

Background: Tonic (Adie's) pupil is characterized by a poor pupillary light reflex, accommodation paresis, strong pupillary response to near stimuli, and slow redilation of pupil after constriction to near stimuli. Damage to the postganglionic parasympathetic innervation of the intraocular muscles produces this syndrome.

Methods: Case report

Case: We report a 38-year-old woman with bilateral tonic (Adie's) pupils. She was diagnosed as having Vogt-KoyanagiHarada syndrome based on accompanying signs of poliosis and previous history of uveitis, serous retinal detachment, and symptoms suggesting meningoencephalitis.

Conclusion: Bilateral tonic pupil is a rare complication of VogtKoyanagi-Harada syndrome. In patients with tonic pupil, preceding or accompanying symptoms and signs of this syndrome should be sought.

\section{P-102}

\section{A kindred of hereditary adult-onset autosomal dominant leukodystrophy in Charlevoix, Quebec}

\section{S. Verreault, M. Langlois, M. Thibault, J.-P. Bouchard (Quebec} City, Quebec), A. Simoes, G.A. Rouleau (Montreal, Quebec)

Background: Most hereditary leukodystrophies are either autosomal or X-linked recessive with an age of onset in infancy or childhood. Adult-onset autosomal dominant leukodystrophies (ADLD) have been rarely reported. We report a new kindred from Charlevoix, Quebec with ADLD.

Methods: Genealogical, clinical, laboratory and neuroradiological data were reviewed in six patients with ADLD evaluated in the last three decades.

Results: Genealogical studies revealed 11 patients with ADLD over four generations in this kindred. Clinical findings consisted of an adult-onset neurological deterioration characterized first by autonomic abnornalities (bladder/bowel dysfunction and orthostatic hypotension), followed by upper motor neurons signs (paraparesis, hyperreflexia and bilateral extensor plantar responses) and abnormal gait. Laboratory investigations were unrevealing including: VLCFA, arylsulfatase $A$, galactocerebrosidase, CSF analysis and NCS/EMG. MRI studies revealed mild cerebral atrophy with symmetrical white matter changes most pronounced in fronto-parietal regions. However, the most striking finding was a severe diffuse spinal cord atrophy not previously reported in ADLD.

Conclusions: Similar clinical and brain imaging findings have been reported in members of an American-lrish family with ADLD, in whom the gene was localized recently on chromosome $5 q 31$. The condition described in our kindred is clinically identical to this syndrome and genetic studies are in progress.

\section{P-102}

\section{Refractory neurosarcoidosis responding to infliximab}

\section{J.Pettersen, M.D. Hill, R. Bell, D. Zochodne (Calgary, Alberta)}

Background: Sarcoidosis is an idiopathic inflammatory disease characterized by granulomatous infiltration of multiple organ systems including the brain. Recent evidence suggests that TNFalpha plays a pivotal role in the pathogenesis of this disease. Case reports have demonstrated a beneficial response to infliximab, a chimeric monoclonal human-murine antibody against human TNFalpha, among patients with refractory disease manifesting as skin involvement (lupus pernio), restrictive lung disease, enteropathy or myopathy. To our knowledge, the effects of infliximab on neurosarcoidosis have not been previously reported.

Methods: Case report.

Results: A 46-year-old-man with sarcoidosis affecting the brain, skin, liver and lungs experienced progressive neurological involvement over the preceding two years, despite receiving courses of high-dose steroids, imuran, and cyclosporin. His course was progressive and characterized by confusion, disorientation, aggressive behaviour, right hemiparesis, and concomitant progression of cerebral lesions on MRI. Following a 3-dose course of infliximab $(5 \mathrm{mg} / \mathrm{kg})$ given at weeks 0,2 and 6 , the patient experienced significant recovery manifested as a 10-point improvement on the MMSE, no further aggressive behaviour, resolution of right hemiparesis and reduced lesion volume and surrounding edema on MRI.

Conclusion: Infliximab, an anti-tumour necrosis factor-alpha, may be an effective treatment for refractory neurosarcoidosis.

\section{P-104}

A rare presentation of a rare disease: Neuro-Behcet's in an African woman

\section{J.Pettersen, G. Klein (Calgary, Alberta)}

Background: Behcet's disease, an idiopathic relapsing multisystem vasculitis characterized by recurrent oral ulcers and cooccurrent lesions of the genitalia, skin and/or eyes, has an extremely low prevalence in North America (0.5-1:500,000) and Africa (case reports). Nervous system involvement occurs in less than $20 \%$ of cases and comprises the presenting complaint in only $5 \%$ of cases. 
Methods: Case report.

Results: A 34-year-old Sudanese woman with a five month history of fronto-temporal headaches, weight loss and fatigue was admitted to hospital with associated fever as well as genital and oral ulcers. Extensive investigations for infectious, neoplastic, and autoimmune etiologies were negative. She returned to hospital one month later with similar symptoms, which were found to be strongly steroid responsive. Recurrent episodes of symptoms, including associated left-sided pyramidal weakness, occurred during steroid taper and subsequently remitted with the re-initiation of steroids. The presence of recurrent genital and oral ulcerations in conjunction with characteristic MRI findings of parenchymal "brainstem-plus" involvement and the steroid-responsiveness of both clinical and radiographic findings led to a diagnosis of probable neuro-Behcet's disease.

Conclusion: Although rare, Behcet's is a treatable disease that should be considered in the differential diagnosis of patients with oral and genital ulcers and progressive neurological symptoms.

\section{P-105}

\section{Neurological manifestation as the presenting complaint in a patient with sarcoidosis}

\section{J.Pettersen, G. Klein (Calgary, Alberta)}

Background: Sarcoidosis is an idiopathic multi-organ granulomatous disease. Neurological involvement occurs in less than $15 \%$ of patients and is very rarely the earliest or only manifestation of disease. While characteristic features of neurological involvement can suggest sarcoidosis, systemic findings (e.g., hilar lymphadenopathy) aid in confirming the diagnosis.

Methods: Case report.

Results: A 29-year-old woman presented with an eight-month history of severe progressive headaches with features suggestive of raised intra-cranial pressure and associated episodes of expressive aphasia. There was an associated $40 \mathrm{lb}$ weight gain, as well as secondary amenorrhea, fatigue, chills and night sweats. Aside from equivocal features of papilledema, the remainder of her examination was normal. Neuroimaging revealed diffuse infiltration and associated edema of the leptomeninges, hypothalamus and bilateral temporal lobes. Bilateral hilar lymphadenopathy was evident on chest $\mathrm{x}$-ray and was confirmed by CT imaging. Blood work was significant for mildly elevated serum calcium, but normal ACE and indicators of pituitary-hypothalamic function. Biopsy of hilar lymph tissue revealed noncaseating granulomatous lesions, consistent with sarcoidosis. There was significant clinical and radiographic improvement following administration of dexamethasone.

Conclusion: Neurosarcoidosis is a rare but treatable disease and should be considered in patients with MRI changes of infiltration of the leptomeninges and hypothalamus.

\section{P-106}

An acute amnestic syndrome in a patient with type 1 diabetes and suspected hypoglycemia

\section{K. Pope, R. Harland, S. Spacey, J. Martzke, (Vancouver, British Columbia)}

Background: Previous case reports have described amnestic syndromes as a sequela to severe hypoglycemic events. We present a case of a type I diabetic who presented with an isolated amnestic syndrome.
Methods: A case report.

Results: A 39-year-old information systems consultant was brought to the emergency department with a three-day amnestic syndrome first noted on awakening. His presentation was characterized by three months of retrograde amnesia and dense anterograde amnesia. Neurologic examination was otherwise normal. His past medical history included type 1 diabetes mellitus since age 18 . Two weeks earlier he had documented hypoglycemic coma with full recovery.

A non-contrast CT head scan, EEG, and lumbar puncture were normal except for a mildly elevated CSF protein. Herpes simplex PCR was negative. MRI one month later was normal. Neuropsychologic testing two weeks after symptom onset identified a persistent discrete deficit in verbal retention and retrieval. All other parameters were measured as average or above average.

Conclusions: This patient experienced an injury to his dominant memory systems resulting in a pure amnestic syndrome. No vascular, epileptic, paraneoplastic or infectious etiology was identified. Several clues support our patient being at risk for occult nocturnal hypoglycemia. We propose that our patient had an undocumented profound nocturnal hypoglycemic episode resulting in an acquired amnestic disorder.

\section{P-107}

Collet-Sicard syndrome from metastatic melanoma to the skull base

\section{E. Roger, M. Veilleux, (Montreal, Quebec)}

Background: The Collet-Sicard syndrome refers to an ipsilateral palsy of the lower cranial nerves (IX to XII) without the associated sympathetic palsy seen in Villaret's syndrome. The Collet-Sicard syndrome has been associated with infectious processes, trauma and metastatic spread of the skull base.

Methods: We will present a very interesting case of Collet-Sicard syndrome from metastatic melanoma presenting initially only as occipital pain and hypoglossal palsy.

Results: We report a 61-year-old lady with a history of melanoma, who presented to the neurology service for left occipital headache of one month duration. She had hemiatrophy of the left tongue with complete hypoglossal paresis to the left. Imaging showed a skull base lesion obliterating the hypoglossal canal. Lesions were found in the lung and diffusely along the spine. The lung biopsy was positive for melanoma. She received local radiotherapy to the skull base but her clinical status unfortunately deteriorated rapidly as she developed dysphagia, dysphonia, and severe spinal pain. She eventually succumbed to an aspiration pneumonia seven weeks after admission.

Conclusion: The metastatic spread of malignant melanoma to the skull base causing lower cranial nerve palsies (Collet-Sicard syndrome) as the sole presenting feature is exceptionally rare. The prognosis is extremely poor, despite aggressive management.

\section{P-108}

\section{A case of multiple recurrent ipsilateral Bell's palsy}

\section{S. Savedia-Cayabyab, S.D. Spacey (Vancouver, British Columbia)}

Background: Bell's Palsy (BP) is a common syndrome of acute lower motor neuron facial nerve palsy. Its rate of recurrence is low 
(5-7\%) with few having an ipsilateral event more than twice. Recent studies have implicated HSV as the most likely underlying cause. We report a case of a 48 -year-old who presents with four episodes of recurrent BP.

Methods: The patient was examined and investigated with neuroimaging, lumbar puncture and serology.

Results: Each episode began with an earache 48-72 hours prior to an acute onset of facial paralysis. The BP was associated with ipsilateral distortion in taste and hearing and increased tearing. Herpetic vesicles were never observed. The patient had a history of herpes zoster on the ipsilateral side five years prior. Recovery was nearly complete without treatment after the first two episodes but incomplete after the third even with a course of prednisone. With the fourth episode acyclovir was initiated. We present the results of neuroimaging, CSF studies and serology.

Conclusion: We report a case of a man who presents with four episodes of ipsilateral BP. This is worth reporting given the rarity of recurrence. The most likely etiology of recurrent $B P$ in this case is $\mathrm{HZV}$ infection.

\section{P-109}

\section{Botulinum toxin type $A$ in focal hyperhidrosis}

\section{N.J.Spratt, J. Blackie (Australia)}

Background: Botulinum toxin type A has been used as treatment for focal hyperhidrosis. Published experience to date is limited, and optimum dosing regimes and method of administration remain unclear. We describe our experience in 10 patients with combinations of palmar, plantar and axillary hyperhidrosis.

Methods: Botulinum toxin type A (Botox, Allergan) was administered to the palms or soles after administration of local nerve blocks; local anaesthesia was unnecessary for axillae. Dose (30-75 $\mathrm{MU} / \mathrm{site}$ ), and number of injection sites were titrated according to response - both subjective, and visualised using the iodine/starch test.

Results: All patients reported response to treatment, however duration of response varied considerably. In contrast to the published literature, palmar and plantar responses were of shorter duration than those after axillary injection. There were no reports of significant weakness.

Conclusions: Botulinum toxin is an effective treatment for severe forms of focal hyperhidrosis. Pain associated with injection of palmar and plantar regions can be prevented by the administration of local nerve blocks. Multiple injection sites, provide a more even response and minimise the risk of weakness.

\section{P-110}

\section{White matter demyelination in cryptococcal meningitis}

\section{Thyagarajan, E. Kat, J. Ravindran, (Adelaide, Australia)}

A young male presented with cough and non-specific symptoms. Papilloedema was found on examination. CT and MRI revealed changes consistent with widespread patchy, white matter demyelination and the patient was considered to have acute disseminated encephalomyelitis. However, CSF examination revealed cryptococcal meningitis in addition to a marked lymphocytic pleocytosis and increased protein. The white matter changes began to resolve even before specific treatment for cryptococcal meningitis was instituted, excluding the possibility that the white matter lesions were due to direct infection of the brain. The patient was subsequently found to have common variable immunodeficiency. We present this very unusual clinical and radiological presentation to indicate that white matter immune response may occasionally be part of the clinical picture of cryptococcal meningitis.

\section{P-111}

Varicella zoster meningoencephalitis in the immunocompetent patient

\section{S.H.Tisch, J.R. Fulgham (Rochester, USA)}

Background: Varicella zoster virus (VZV) is an unusual cause of meningoencephalitis in the immunocompetent host and usually occurs in older patients, is preceded by skin rash and is associated with large vessel cerebral vasculitis. We report a case of meningoencephalitis without prior rash or evidence of cerebral vasculitis in an immunocompetent host. The literature is reviewed.

Methods: A 54-year-old male presented with one week of excessive somnolence and global headaches. On examination the patient was febrile, lethargic and had mild expressive aphasia. Serum antibodies to HIV 1 and 2 were negative. The CSF examination showed 477 leukocytes $(80 \%$ lymphocytes, $3 \%$ neutrophils), 38 erythrocytes, protein $456 \mathrm{mg} / \mathrm{dL}$, glucose 122 $\mathrm{mg} / \mathrm{dL}$. PCR for VZV DNA in CSF was positive. PCR for herpes simplex virus (HSV) was negative. IgG antibodies against VZV were positive in both serum and CSF in rising titers. MRI and MRA of the head were normal. The patient recovered fully with intravenous acyclovir therapy.

Results: N/A

Conc/usions: VZV may cause an atypical meningoencephalitis in immunocompetent patients without prior skin rash or evidence of cerebral vasculitis. The clinical picture may resemble HSV encephalitis. Positive CSF VZV PCR confirms the diagnosis. Early recognition is important because specific antiviral therapy may be instituted.

\section{P-112}

\section{Hemisensory syndrome: poor diagnostic yield and a nearly} uniformly benign prognosis

\section{Toth, T. Lad (Saskatoon, Saskatchewan)}

Objective: To describe the diagnostic yield and prognosis for patients with hemisensory syndrome.

Background: The utility of diagnostic procedures and outcome of patients with exclusive hemibody complaints with only subjective sensory abnormalities (hemisensory syndrome) on examination is unknown.

Methods: We prospectively identified patients with hemisensory syndrome from 1998-2001. Diagnostic procedures were analyzed for sensitivity. Clinical follow-up was performed.

Results: Thirty-two patients, 23 females, of age $35 \pm 11$ years were identified. The hemisensory syndrome occurred on the left side in 21 cases. Past history was remarkable for migraine in six, and prior psychiatric diagnosis in 18 patients. Neuroimaging demonstrated diagnostic abnormalities representing ischemic etiology in two cases. CSF examination demonstrated non-specific changes in one case. Electrophysiology, systemic radiological imaging and blood testing 
revealed no diagnostic abnormalities. Nineteen patients had persistent short-term complaints at $7 \pm 5$ days, and four patients had persisting complaints at $17 \pm 6$ months. One patient had a stroke seven months after presentation. Other diagnoses at long-term follow-up included migraine with aura (4 cases), migraine without aura (2 cases), and psychiatric diagnoses (4 cases).

Conclusions: Diagnostic yield in hemisensory syndrome is poor, and prognosis is almost uniformly benign.

\section{P-113}

Posterior cerebral artery infarct presenting with acute encephalitic syndrome with leptomeningeal and gyriform enhancement on MRI, misdiagnosed as herpes simplex encephalitis

C. Voll, C. Toth, F. Pirouzmond (Saskatoon, Saskatchewan)

Background: Leptomeningeal/gyriform enhancement is usually infective or neoplastic in origin and is due to disruption of the blood brain barrier.

Methods: Case report.

Results: A 79-year-old male presented with an acute confusional syndrome and low grade fever. Examination revealed no meningism and a left homonymous hemianopia (HHA). Cranial MRI demonstrated leptomeningeal and gyriform enhancement and punctate hyperintensities in the right parieto-occipital region, extending to the hippocampus, diagnosed as most consistent with viral meningoencephalitis. EEG showed polymorphic right hemispheric delta, but no epileptiform discharges. There was CSF lymphocytosis, and treatment with acyclovir was initiated for presumptive herpes simplex viral (HSV) encephalitis. CSF HSV polymerase chain reaction was, however, negative and acyclovir was discontinued on day 3. Carotid/cerebral angiography showed $95 \%$ stenosis of the right internal carotid artery which supplied only the right posterior cerebral artery. A right carotid endarterectomy was performed and the patient recovered uneventfully but with persistent left HHA.

Discussion: Leptomeningeal and gyriform enhancement on MRI in association with CSF lymphocytosis led to an initial misdiagnosis of a probable infective meningoencephalitic process. Leptomeningeal and gyriform enhancement on CT and MRI may occur in association with acute stroke due to ischemic disruption of the blood brain barrier leading to diagnostic confusion.

\section{P.114}

Palinacousis in association with a right parietal glioblastoma multiforme

\section{C.L.Voll, M. Hickie, M. Kelly (Saskatoon, Saskatchewan)}

Background: We report palinacousis (auditory perseveration) in a patient with a right parietal glioblastoma multiforme.

Methods: Case report.

Results: Four days prior to presentation, the patient described hearing "echoing of voices" in his left ear. Words spoken by others would recur for seconds to minutes. Sounds other than speech were not affected. Hearing was normal and general neurological examination was normal. EEG demonstrated right hemispheric polymorphic delta. Treatment with phenytoin resulted in complete resolution of the palinacousis. Cranial MRI demonstrated a $2 \mathrm{~cm}$ enhancing cortically-based right parietal lesion with minimal surrounding edema. Craniotomy was performed with total resection of the tumor. Biopsy confirmed a glioblastoma multiforme.

Discussion: Palinacousis has been described previously in association with lesions of the temporal lobe, temporo-parietal lobe and medical geniculate nucleus. Our case is unique in that a discrete lesion was confined to the parietal lobe, a localization not previously reported for palinacousis. Musical hallucinosis, similar to palinacousis has been described with left parietal lesions and a common pathway may underlying musical hallucinations and palinacousis. The improvement in our patient's symptoms after treatment with phenytoin favours an epileptic etiology. Palinacousis should be recognized as a sign of organic disease and not confused with auditory hallucinations from psychosis.

\section{P-115}

\section{Late-onset multiple sclerosis}

\section{G. Vorobeychik, D.W. Paty and the UBC MS Clinic (Vancouver, British Columbia)}

Objective: To evaluate the clinical course of multiple sclerosis (MS) in individuals who had the onset of MS at age 60 or older.

Background: Studies of the natural history of late onset MS with inclusion of standardized longitudinal disability measurements are of particular interest.

Methods: Patients with onset of clinically definite MS after age $60(n=17)$ with complete clinical information on the clinical course from the MS Clinic at University of British Columbia Site Hospital computerized database (MS-COSTAR) were included in the study.

Results: The mean duration of observation was $10.9 \pm 4.5$ years, the mean age of MS onset was $63.9 \pm 4.9$, range 60 to 73 years. There were eight cases (47\%) of primary progressive MS (PPMS) course. To date, seven $(77.8 \%)$ of nine subjects have developed secondary progressive MS (SPMS), six (85.7\%) of seven patients did it within 10 years from onset (mean time to SPMS was $7.8 \pm 3.8$ years). All patients with PPMS and five (56\%) of nine patients with relapsingremitting or SPMS reached confirmed Expanded Disability Status Scale $($ EDSS $)=6.0$ during observation period.

Conclusions: A primary progressive course and rapid progression to disability is more common in late onset MS than in young adults.

\section{P-116}

Variant CJD: development of a national co-ordinated care programme

\section{B. Weller, R. Will, G. Mclean (Edinburgh,UK).}

Background: Variant CJD is a devastating illness described in 1996 with 113 cases to date in the UK. The Douglas report (1999) highlighted major inadequacies in the care provided to VCJD sufferers and as a result a National CJD Care Unit has been formed. National care co-ordinators visit all cases of $\mathrm{VCJD}$, to provide information to relatives and health professionals, to advise on co-ordination of necessary services and on symptom management. A care fund is administered by the care co-ordinators to overcome deficiencies in services which cannot be provided by local authorities.

Method: Case record audit of the first 22 cases referred to the National CJD Care Unit.

Results: The mean age was 26 with a mean disease duration 
following referral of 5.2 months. All patients had significant functional disability at referral. All had gait disturbance with ataxia (five bed or chair-bound, ten walking with assistance, eight independent) and cognitive disability (MMSE 19/30 n=11) and the majority required assistance with ADLS. Sixteen of the 22 patients were cared for at home. All patients became totally dependant, with loss of mobility, swallowing and continence and all developed behavioural changes, most commonly aggression or anxiety. Other symptoms and medications used are described along with the most frequently required equipment and services. Uses of the National Care Fund are also documented.

Conclusion: Results of this study illustrate the rapidly progressive nature of this illness, particularly after diagnosis and show that severe disability and troublesome symptoms are already present in all patients at this stage. For patients to return home input from a wide range of health professionals must be co-ordinated rapidly with provision of appropriate equipment and house adaptations. Accumulation of data relating to the course of this illness after diagnosis will enable prediction of the care requirements of vCJD patients and their more timely provision and enable a more systematic use of medication for symptom relief.

References:

1. Will RG, Ironside JW, Zeidler M, et al. $1996 \mathrm{~A}$ new variant of Creutzfeldt-Jakob Disease in the UK. Lancet, 347; $921-923$.

2. Department of Health Monthly Figures, National CJD Surveillance Unit, Dec 2001

3. Douglas M, Campbell H, Will RG, Feb 1999. Patients with new variant CJD and their families: care and information needs. Website of the National CJD Surveillance Unit, Edinburgh. (www.cjd.ed.ac.uk)

\section{P-117}

Nitrous oxide - it's no laughing matter

\section{E.M. Whitham, M. Kiley, P. Thompson (Adelaide, Australia)}

Two cases are illustrated of the adverse neurological consequences of nitrous oxide, both therapeutic and recreational.

Case one of a female utilizing $\mathrm{N}_{2} \mathrm{O}$ for the dressing of painful, recurrent ulcers, who developed a rapid 10 day onset of numb, clumsy hands and progressive unsteadiness of gait. The other a young man with recreational usage ("nagging"), presenting with a two day history of peripheral tingling and numbness of hands and feet, with unsteadiness of gait.

Of the two cases, one had low $\mathrm{B}_{12}$ levels, but no haematological sequelae noted; the other normal $B_{12}$ levels but a macrocytosis. Nerve conduction studies revealed peripheral slowing of motor and sensory potentials.

The postulated mechanisms of effects upon the $B_{12}$ regulated enzymatic processes are described and treatment recommendations.

\section{P-118}

Intravenous lignocaine infusion for the treatment of analgesic rebound headache

\section{D.R. Williams, R.J.Stark, (Melbourne, Australia)}

Background: Patients with analgesic rebound headache are difficult to treat, especially when the doses of analgesia are substantial. We have previously shown that intravenous lignocaine infusion is useful in maintaining pain control while the offending analgesic agent is withdrawn in these patients. The published data on long-tern efficacy of this treatment is limited.
Methods: Retrospective survey of consecutive patients admitted for lignocaine infusion for treatment of chronic daily headache, with analgesic abuse.

Results: Sixty-eight patients (58 women) received lignocaine infusions for an average of 8.6 days. $88 \%$ of patients had a history of migraine headaches. In $84 \%$ of patients, codeine was the agent implicated in the analgesic rebound headaches (average $1050 \mathrm{mg} /$ week) and $22 \%$ used ergotamine containing medications (average $18 \mathrm{mg} /$ week). $28 \%$ frequently used injected opioids. At discharge $89 \%$ of patients reported that their daily headache was absent or improved, and the analgesic agent was withdrawn successfully in $98.5 \%$. At six month follow-up $69 \%$ of patients $(77 \%$ of migraneurs) reported that their daily headache was absent or improved, and $73 \%$ of patients ( $78 \%$ of migraneurs) remained free of the offending analgesic agent.

Conclusions: Intravenous lignocaine is a useful treatment in the management of analgesic rebound headache. The benefits of the program last for at least six months. The treatment appears to be most efficacious in patients with transformed migraine.

\section{MOVEMENT Disorders}

\section{P-119}

\section{A comparison of the duration of action and safety margins of botulinum toxin types $A$ and $B$ in mice}

\section{K.R. Aoki, (Irvine, USA)}

Background: This study compared the duration of muscle weakening and safety margin of the recently-approved botulinum toxin type B (BTX-B) to BTX type A (BTX-A).

Methods: Mice received a single hind limb intramuscular (IM) injection of BTX-B (Myobloc ${ }^{\mathrm{TM}}$ ) $\left(1-150 \mathrm{U} / \mathrm{kg}\right.$ ) or BTX-A (Botox ${ }^{\text {(i) }}$ or Dysport $\left.{ }^{\circledR 0}\right)(1-200 U / k g)$. Muscle weakening efficacy was assessed by an observer masked to treatment using the digit abduction scoring assay (DAS). DAS IM-ED 50 values and median IM lethal doses were calculated from regression equations to determine safety margins (IM-LD $\mathrm{L}_{50} / \mathrm{IM}-\mathrm{ED}_{50}$ ).

Results: BTX-A (Botox ${ }^{\circledR}$ ) at $29 \mathrm{U} / \mathrm{kg}$ and BTX-B at $67 \mathrm{U} / \mathrm{kg}$ produced comparable peak DAS scores of approximately four indicating maximal muscle weakness, but the duration of BTX-A was longer, with a mean DAS score of 2 at day 14 compared to a score of 0 (return to baseline) with BTX-B. The safety margin was largest for BTX-A (Botox ${ }^{\boxplus:}$ 13.9), followed by the other preparation of BTX-A (Dysport ${ }^{\circledR}: 7.6$ ), and finally BTX-B (Myobloc ${ }^{\mathrm{TM}}: 5.4$ ).

Conclusions: BTX-A had a longer duration than BTX-B, a finding similar to all published studies that have directly compared these two serotypes. The different safety margins of the three products demonstrate that neurotoxin preparations are not comparable by simple dose ratios and may indicate different clinical profiles.

\section{P-120}

A patient specific brain atlas of subcortical electrophysiologic observations

\section{K. Finnis, Y. Starreveld, A. Parrent, T. Peters (London, Ontario)}

During stereotactic functional neurosurgery, surgical targets are approximated using standardized coordinates scaled to anatomical 
landmarks identified on preoperative brain images. Many structures targeted in this way are functionally distinct but not discernable on magnetic resonance images (MRIs). While electrophysiologic tissue characterization provides a wealth of knowledge, interpatient anatomical variability precludes incorporation of these data into the planning of subsequent cases.

We present a database of intraoperatively recorded human subcortical electrophysiology that addresses anatomic and physiologic variability among Parkinsonian patients. Electrophysiologic data obtained from 122 procedures were standardized using a multi-parameter coding system and annotated to their respective MRIs at the appropriate position in stereotactic space. Using a nonlinear image registration algorithm, the patient images were warped to a MRl reference brain. The annotated functional data were then transformed into reference brain coordinates using the displacement grids generated by the algorithm.

When viewed within our visualization software, clustering of interpatient physiologic responses within the diencephalon and mesencephalon is observed. These data may be registered to a preoperative patient MRI enabling the delineation of surgical targets and somatotopic organization prior to surgery. The database is expandable, fully searchable, and provides a visual 3D representation of the functional organization of the deep brain.

\section{P-121}

\section{A novel presentation of Friedreich's ataxia in a compound heterozygote}

\section{S.D. Spacey, B.I. Szczygielski, K. Selby, J. Hukin, T.P. Snutch} (Vancouver, British Columbia).

Background: Friedreich's ataxia (FA) is an autosomal recessive condition, characterized by a slow progressive ataxia. $94 \%$ of cases result from a trinucleotide repeat expansion in the Frataxin gene. Compound heterozygosity accounts for the majority of the remaining cases in which a point mutation is found on the unexpanded allele. We present the clinical features and genetic analysis of a compound heterozygote patient who presented acutely with FA.

Methods: A previously healthy 10-year-old Malaysian boy presented with fever, lethargy, headaches, dysarthria, vertigo and ataxia developing over a one week period. The patient was investigated for causes of ataxia including genetic analysis of the Frataxin gene.

Results: The patient was extensively investigated but found to have normal CSF, VLCFA, arylsulfatase A, phytanic acid, vitamin E and $\mathrm{B} 12$, cholesterol, triglycerides and hexosaminadase A\&B. Genetic analysis demonstrated an expanded GAA repeat in the Frataxin gene on one allele only. The exons in the Frataxin gene on the unexpanded allele are currently being sequenced. The patient's sister subsequently presented with ataxia and was diagnosed as a compound heterozygote based on genetic analysis.

Conclusion: FA is an autosomal recessive condition. Less than $6 \%$ of cases are compound heterozygotes. We describe an acute presentation of FA, a novel phenotype, in a patient who is a compound heterozygote.

\section{P-122}

Mutation analysis of the sodium/hydrogen anion exchanger gene (NHE5) in familial paroxysmal kinesigenic dyskinesia

S.D. Spacey, B.I. Szczygielski, J. McRory, T.P. Snutch (Vancouver, British Columbia), N.W. Wood (London, England)

Objective: To investigate the role of the $\mathrm{Na}^{+} / \mathrm{H}^{+}$anion exchanger gene (NHE5) in familial paroxysmal kinesigenic dyskinesia (PKD).

Background: Familial PKD is an autosomal dominant condition characterized by attacks of dystonia or chorea triggered by sudden movements. Recently two separate loci for PKD, EKD1 and EKD2, have been mapped to chromosome 16 but the causative genes have not been identified. The $\mathrm{Na}^{+} / \mathrm{H}^{+}$anion exchanger gene (NHE5) involved in regulating intracellular $\mathrm{pH}$ lies in the EKD2 region. Abnormalities in its function could potentially cause paroxysmal neurological disease.

Method: The intron/exon boundaries and the exons of the NHE5 gene were sequenced in a family with familial PKD linked to the EKD2 locus.

Results: There were no mutations in the exons or the intron/exon boundaries of the NHE5 gene.

Conc/usion: PKD is a paroxysmal movement disorder. Many of the paroxysmal neurological conditions are the result of gene mutations which influence neuronal excitability. Reports of a mutation in the mouse NHEl gene producing a seizure disorder confirms that genes regulating membrane $\mathrm{pH}$ can cause paroxysmal neurological conditions. The NHE5 gene regulates neuronal intracellular $\mathrm{pH}$ and could causes paroxysmal disease from abnormal regulation. No mutations were identified in the exons or intron/exon boundaries in familial PKD linked to the EKD2 region. The NHES gene is unlikely to be the causative gene for familial PKD.

\section{P-123}

Inter-rater reliability of the ICARS (International Cooperative Ataxia Rating Scale)

\section{E. Storey, K. Tuck, R. Hester, A. Hughes, A. Churchyard}

(Melbourne, Australia)

Background: Clinical rating of global ataxia severity is typically subjective, although validated laboratory measures exist for individual components such as stance or eye movements. The ICARS was introduced in 1997 to provide a standardised clinical rating system for ataxia treatment trials. As part of our study into the neuropsychological accompaniments of inherited ataxias, we addressed the inter-rater and intra-rater reliability of the ICARS and its component subscores.

Methods: Eleven subjects with spinocerebellar ataxia (SCA) type 1, one with SCA2, 10 with Friedreich's ataxia (FA), and four controls underwent the ICARS assessment. Two neurologists, blind to the diagnoses, scored the videotaped assessments. One (ES) had scored the assessments, unblinded, at the time of taping up to 10 months earlier. Non-parametric correlations (Kendalls tau; W) were calculated using SPSS.

Results: W was 0.95 for inter-rater and 0.99 for intra-rater total scores. W for inter-rater subscale scores were 0.99 for posture and gait, 0.97 for kinetic functions, 0.79 for speech, and 0.65 for oculomotor. 
Conclusions: The ICARS has good inter-rater and intra-rater reliability. The subscale Ws of $0.65-0.99$ compare favourably with those for UPDRS (motor) items (0.62-0.95), for example. Descriptor modification and teaching video standardisation may improve reliability further.

\section{P.124}

Dark dentate disease (DDD) - a novel dominantly-inherited spinocerebellar ataxia

E. Storey, M.A. Knight, S.M. Forrest, J. Dixon, H. Rundle, M. Gardner (Melbourne, Australia)

Background: A minority of Australian pedigrees with dominant spinocerebellar ataxia (SCA) are accounted for by SCAs 1,2,3,6 or 7. This prompted a search for SCA pedigrees with novel phenotypes, resulting in this description of DDD.

Methods: Thirteen definitely affected members of this AngloCeltic pedigree were examined systematically.

Results: Onset age ranged from 19-64 years. The initial symptom was dysarthria in nine, gait ataxia in two, and both in two. Progression was slow, with one wheelchair-bound after 40 years of symptoms. The typical clinical picture was of pure ataxia, with minor pyramidal signs in five. Persistent nystagmus was not seen, but hypermetric saccades occurred in 11. Palatal tremor occurred in 10 , and an unusual dysphonia resembling spasmodic dysphonia was superimposed on cerebellar dysarthria in eight. Sensory findings were essentially normal, and NCS were normal in three. CT showed dentate calcification in $8 / 8$ with pallidal calcification in only two. MRI showed isolated pancerebellar atrophy with dark dentate signal in 4/4. Direct testing and linkage analysis have excluded SCAs 1-17.

Conclusions: DDD is a novel dominant SCA, characterised by dysphonia, palatal tremor and dentate calcification. It is sufficiently distinctive that the disorder is probably rare.

\section{P-125}

Time required to program deep brain stimulators (DBS) in thalamus, globus pallidus internus (GPi), and subthalamic nucleus (STN)

Karen Hunka, Oksana Suchowersky, Zelma H.T. Kiss, (Calgary, Alberta)

Background: DBS are increasingly used to treat movement disorders. This study was to prospectively determine the time required to program patients with DBS electrodes for essential tremor (ET) and Parkinson's disease (PD).

Design/Methods: Nine patients two with ET, seven with PD) who were programmed (by $\mathrm{KH}$ ) in Calgary since March 200I were studied. Sequential trials of monopolar then bipolar stimulation (starting at $135 \mathrm{~Hz}, 0.06 \mathrm{~ms}$ pulse width) were initiated 4-6 weeks postoperatively in the "ON" state. After a setting was found which suppressed symptoms with the fewest adverse effects, voltage was gradually increased.

Results: Both ET patients had unilateral thalamic-DBS, six PD patients had bilateral STN-DBS and one patient had a unilateral GPiDBS. All patients showed significant improvement in UPDRS and/or tremor rating scores. Mean programming time was $9.13 \mathrm{~h}$ (range 9.0-9.25) for thalamic-DBS, $10.75 \mathrm{~h}$ for GPi-DBS and 14.25 h (range 8-18.50) for STN-DBS. The average number of visits per patient was 7.9 (range 4-12) and programming was performed over 12.4 (range 5-22) weeks.

Conclusions: Time required for programming of STN-DBS was higher compared to thalamic and GPi-DBS, likely reflecting the bilateral nature of the STN procedure. This must be factored into budget projections when developing a movement disorders surgery program.

P-126

Writer's cramp in an Australian pedigree with DYT1 dystonia D. Thyagarajan, (Adelaide, Australia)

I present the clinical features of the first molecularly characterized pedigree of DYTI dystonia in Australia. In this Caucasian family with no Jewish ancestry, the presentation in the proband was writer's cramp. Examination revealed no other evidence of limb dystonia at the age of fifteen. There has been no progression over two years and the patient responded partially to trihexyphenidyl. The patient's sister, a few years older also had milder writer's cramp.

Examination revealed mild scoliosis but no other evidence of limb dystonia. After two years of follow-up she has developed nild foot dystonia. There has been no response to trihexyphenidyl. Clinical and molecular genetic data of other family members are presented in this large pedigree. Writer's cramp alone is an unusual presentation of DYT 1, but the possibility of a mutation in the DYT1 gene should be considered in patients presenting with this type of task-specific dystonia, particularly if there is a family history.

\section{P-127}

Adult-onset leukodystrophy and palatal myoclonus: adult Alexander disease?

D. Thyagarajan (Adelaide, Australia); E. Storey, E. Byrne (Melbourne, Australia).

We present a pedigree with adult-onset spastic diplegia in an apparent autosomal dominant inheritance. The proband had striking palatal myoclonus and a disorder of eye movement (video). MRI revealed white matter signal changes consistent with a leukodystrophy. Her son was minimally affected with mild intellectual impairment but cerebral MRI was abnormal in a similar fashion.

Investigations ruled out recessive leukodystrophies. Sequencing of the GFAP gene is in progress. We discuss the sequencing results, review the literature on the rare dominantly-inherited leukodystrophies and the likelihood that this is another example of adult-onset Alexander disease.

\section{P-128}

\section{Donepezil in patients with Parkinson's disease and dementia}

\section{S. Trepanier, M. Panisset (Montreal Quebec)}

The basis for cognitive patients with Parkinson's disease (PD) deficits is still unclear. Dopaminergic and cholinergic systems have been implicated. The goal of this study was to assess the effect of the cholinesterase inhibitor donepezil on cognitive functioning of patients with PD. Six patients were given donepezil and six control 
patients matched by age and duration of disease were selected. All patients with $\mathrm{PD}$ and mild dementia were given tests measuring attention (trail test, digit span), memory (ADAS-Cog, visual span, Benton) language, praxis, executive functions (letter and number sequences), facial recognition and depression scale (GDS). Patients were evaluated at baseline and a mean of 5.83 months later. The donepezil group improved only on the ADAS-cog test. There was no other difference on the neuropsychological test between group. Of the six patients tried on donepezil, four did show an improvement that was significant to the caregiver and one was deemed stable. This study shows that donepezil can induce a mild improvement in cognition of patients with PD, and dementia cognition significantly in patients with $\mathrm{PD}$ and dementia.

\section{P-129}

Quantitative analysis of ballistic movements in inherited ataxia

\section{K. Tuck, L. Rudolph, R. Stephens, R. Hester, E. Storey (Melbourne,} Australia)

Background: The purpose of this project was to quantitatively analyse ballistic arm movements in subjects with inherited ataxia. A specific recording device was designed and built for this study, and a detailed analysis to determine the most powerful parameter for separating controls from ataxic subjects was undertaken.

Methods: The recording device is a potentiometer mounted in a swivelling moulded arm support. A horizontal bar with lights positioned at 10,20, and 30 degrees left and right of centre is aligned with the subject. The lights operate in a pseudo random order without cue. The subject is instructed to align with the light quickly and accurately. Using signal processing software, parameters including: time to initiate movement, peak velocity, mean velocity, peak acceleration, velocity curve peak numbers, and velocity symmetry variance were analysed.

Results: Interim analysis of 19 ataxic patients and 13 matched controls identified four significant parameters. Mean velocity, velocity symmetry variance, and velocity curve peak numbers were all significant at 10 degrees. Time to initiate movement was significant for all positions.

Conclusion: Preliminary results suggest the ballistic tracking device significantly differentiates between ataxic subjects and matched controls.

\section{NEUROPHYSIOLOGY}

P-130

Clinical, electrophysiological and neuro-imaging features of spinal decompression sickness

J.Y.Chu, A.W. Evans (Toronto, Ontario)

Background: Spinal cord injury due to decompressive sickness is a rare neurological complication from diving. It frequently results in significant sensory and motor dysfunction and may cause severe neurological sequelae. The clinical and electrophysiological features of a successfully treated case of spinal decompression sickness will be presented.

Methods: Case report.

Results: A 65-year-old man developed acute onset of paraparesis of his lower limbs with numbness of his left leg following a 90 feet dive. His clinical examination demonstrated moderate lower limb weakness (4/5) with loss of pain and temperature sensation to T12 level. Vibration sense was also impaired in the toes. Plantar response was extensor on the left and flexor on the right. His initial MRI of thoracic spine showed increased signal at T-10 which could be due to microinsult. His lower limb SSEP showed prolongation of central conduction time (T12-P37 inter-peak latency) on the left. He underwent immediate hyperbaric oxygen treatment and demonstrated progressive improvement in his lower limb sensory and motor functions over the next four months. Follow-up MRI two months after the event showed resolution of the initial abnormality. His lower limb SSEP also returned to normal. His subsequent neurological examination showed mild left leg hyper-reflexia with mild loss of pin-prick in the left thigh but he was fully ambulatory.

Conclusions: Spinal decompression sickness should be considered a neurological emergency and could be treated successfully with hyperbaric oxygen if therapy is instituted early. The clinical, electrophysiological and neuro-imaging features of this case will be presented with a review of the literature.

\section{P-131}

Ipsilateral cortical paresis: a key to the anatomy of handedness

\section{L.Derakhshan (Charleston, USA)}

Background: Ipsilateral hemiparesis resulting from hemispheric lesion (traumatic, neoplastic, etc.) is commonly attributed to a hernia, resulting formation of Kernohan's notch.

Method: I present two cases with multiple CT and MR imaging documenting absence of any lesion in the brain except a left subdural (case 1) and epidural (case 2) bleed of the original trauma, leaving the patients with spastic right hemiparesis.

Results: An exhaustive review of literature yielded a large number of similar cases, corroborating a fact rarely attended to before; ie. the ipsilateral weakness is indexed to the subject's handedness. This observation suggests the likelihood of existence of a directionality of interhemispheric traffic with the laterality indexed paresis being due to Von Monakow's diaschisis, resulting in paresis ipsilateral to the lesion in the major hemisphere in right or left handers, as the case might be.

Since a similar situation (ie. laterality indexed weakness) is documented in post callosotomy subjects, it is likely that abovementioned vectorial interhemispheric traffic occurs via the callosum. I will provide substantial data in support of the theory from such fields as high resolution EEG, fMRI, PET, TMS.

Conclusion: Thus, this unusual finding points to the existence of a bihemispherically distributed neuronal ensemble devoted to executive functions.

\section{P-132}

A case of peripheral neuropathy caused by antibodies to myelin associated glycoprotein-response to plasmapheresis

\section{S. Gopinath, A. McDougall (Liverpool, Australia)}

Background: IgM antibodies to myelin associated glycoprotein (MAG) are pathogenic in approximately $50 \%$ of neuropathies caused by monoclonal gammopathies. Various immune therapies have been used in the treatment of these neuropathies with variable responses. 
Methods: A 69-year-old man with a 22-year history of diabetes mellitus presented with six months of increasing leg weakness and parasthesias. Nerve conduction studies showed severe sensorimotor neuropathy with very slow conduction velocities and prolonged distal motor latencies. Serum studies revealed paraproteinemia with elevated titre of anti MAG antibodies (62000 BTU-normal 1000 BTU). Sural nerve biopsy showed a severe loss of myelinated fibres. The remaining myelinated fibres were of small diameter and showed small onion bulb formations.

Results: The patient was treated with plasmapheresis, initially twice weekly then gradually reduced to once fortnightly. There was a significant improvement in patient's symptoms over six months accompanied by similar improvement in nerve conduction studies.

Conclusions: Although plasma exchange has been used as a therapy for monoclonal gammopathies, its effect on neuropathies in controlled studies has been disappointing. Our patient, over I8 months follow-up, has shown stable improvement with plasmapheresis.

\section{P.133}

Semi-quantitative analysis of intraoperative EMG during complex cervical spine surgery

\section{A. Krassioukov, R. Sarjeant, E. Green, G. Bastaldo, M. Fehlings (Toronto, Ontario)}

Background: Intraoperative monitoring of spontaneous EMG activity may enhance the safety of complex cervical spine surgery. However, the quantitative analysis of these recordings represents an unresolved issue and the correlation with postoperative neurological outcomes is unknown. We present a prospective study in 31 consecutive patients to examine this issue.

Objectives: The goal of this study was to correlate the intensity of spontaneous intraoperative EMG (SIEMG) activity and postoperative outcomes in patients that underwent cervical spinal surgery including tumor resection and complex spinal reconstruction.

Methods: Intraoperative upper extremity EMG activity was graded using a 4 point scale, from 0 to 3 (no EMG to excessive EMG activity) based on the frequency, duration and amplitude. New neurological deficits were evaluated postoperatively.

Results: SIEMG activity was observed in 16 subjects (52\%). EMG activity was associated with tumor dissection or nerve root manipulation. In four subjects (13\%) SIEMG was graded as 3. In only one of these four subjects a new postoperative motor deficit was observed. There were no new postoperative motor deficits in subjects with SIEMG activity graded below 3 .

Conclusions: Semi-quantitative intraoperative EMG analysis is a simple technique to predict and possibly to prevent intraoperative damage of neural tissue.

\section{P.134}

Responses of human sensory and motor axons to ischaemia and to hyperpolarizing currents

C.S.-Y.Lin (Sydney, Australia); S. Kuwabara (Chiba, Japan);

C. Cappelen-Smith, D. Burke (Sydney, Australia)

Background: This study was undertaken to compare directly the post-ischaemic behaviour of sensory and motor axons in the human median nerve, focusing on the excitability changes produced by ischaemia and its release and by continuous polarizing DC.

Methods: Accommodation to prolonged subthreshold hyperpolarizing currents of graded intensity and the responses of axons of different threshold during and after ischaemia of different duration were measured.

Results: The changes in threshold during and after ischaemia for 13 minutes were greater and the return to the pre-ischaemic excitability took longer in sensory axons, suggesting that sensory axons have a greater dependence on the electrogenic $\mathrm{Na}^{+} / \mathrm{K}^{+}$pump to maintain resting membrane potential. Sensory axons developed a transient depolarizing threshold shift a few minutes after release of ischaemia. Hyperpolarizing currents of equivalent intensity produced a greater increase in threshold for motor axons than sensory axons and, in studies of threshold electrotonus using graded hyperpolarizing DC of $300 \mathrm{~ms}$ duration, accommodation was greater in sensory than motor axons. These findings are consistent with greater inward rectification (IH) on sensory axons. The postischaemic changes in threshold were not uniform for axons of different threshold, whether sensory or motor, the threshold changes being less prominent for higher-threshold axons. A transient postischaemic depolarisation could be produced in motor axons with ischaemia of 20-minutes duration.

Conclusions: These differences in biophysical properties of sensory and motor axons may produce differences in their susceptibility to dysfunction. This may be a factor in the clinical presentation of neuropathic disturbances.

\section{P-135}

Case report: loss of lower limb SSEPs during carotid endarterectomy

\section{S. Louissi, R. Sarjeant, A. Krassioukov, P. Porter, (Toronto, Ontario)}

Background: Somatosensory evoked potentials (SSEPs) are among the monitoring techniques utilized during carotid endarterectomy to reduce the risk of ischaemic injury. While upper limb SSEPs (ULSSEPs) are thought to be sufficient for monitoring the cerebral cortex, the usefulness of simultaneous lower limb SSEP (LLSSEP) is not well-documented.

Methods: Neurosurgeons perform selective shunting based on significant changes in ULSSEPs during carotid artery crossclamping. Recently we have begun to perform simultaneous monitoring of UL and LLSSEP in all carotid endarterectomy patients.

Results: We present a 79-year-old female with left internal carotid artery (ICA) stenosis who underwent carotid endarterectomy under general anaesthesia. Five minutes after clamping of the artery, there was a complete loss of the LLSSEP cortical response. The surgeon was notified but only shunted twenty minutes later when the ULSSEPs were lost. This resulted in recovery of the right arm cortical response, however the LLSSEP did not improve until the end of the case. Postoperatively, no new neurological deficits were noted.

Conclusions: In this cases, the LLSSEP loss preceded that of the ULSSEP. Although the monitoring standard is ULSSEP during carotid endarterectomy, these observations suggest that the addition of LLSSEP may enhance monitoring in these cases. 


\section{P-136}

Two cases of multiple neuropathy following radial artery harvest

\section{Stefanelli, G. Whelan (Si John's, Newfoundland)}

Background: Radial artery harvesting for CABG has become popular with relatively low complication rates. Radial nerve sensory dysfunction has been documented electrophysiologically. However, more widespread deficits have been described clinically. We report on the clinical and electrophysiological data of two such cases.

Methods: Over one year, repeat clinical examination and NCS/EMG studies were carried out on two patients who developed significant sensory/motor hand and arm deficits post radial artery harvest.

Discussion: In each case the patients had profound dysesthesia and mild to severe weakness in radial, ulnar and in one case median innervated muscles. Electrophysiological studies showed abnormalities in all three nerves in both cases but surprisingly the radial sensory studies were relatively normal. There were residual deficits in both cases at one year follow-up.

Conclusions: Radial artery harvest can result in widespread neuropathy involving radial, median and ulnar nerves, presumably secondary to ischemia. Both these cases occurred in diabetics. Carbamazepine seemed to help the neuropathic pain.

\section{P.137}

\section{The EEG findings in Kufs disease}

\section{Vadlamudi, J. Parisi, B. Westmoreland (Rochester, USA)}

Background: Kufs disease represents the adult autosomal recessive form of neuronal ceroid lipofuscinosis (NCL). We looked at the EEG findings in five biopsy proven cases of Kufs disease and reviewed EEG findings from case reports in the literature.

Methods: The criteria utilized for inclusions were clinical evidence of a progressive neurodegenerative disorder, biopsy proven evidence of Kufs disease, and EEG recordings during the course of the illness. The cases were divided into phenotypes A and B based on Berkovic et al's classification. (Berkovic S.F., et al. Kufs' disease: a critical reappraisal. Brain 1988; 111:27-62)

Results: Those with phenotype $\mathrm{A}$ had generalized atypical spike and slow wave complexes and marked photoparoxysmal responses particularly at low flash frequencies. Those with phenotype $B$ and other miscellaneous cases had generalized slowing, focal sharp waves and quasi-periodic slow waves maximal over anterior head regions.

Conclusions: In this rare condition, the EEG may guide investigations towards confirmation with a pathological diagnosis, as well as differentiating various phenotypes of Kufs disease. More research is needed to understand the inheritance, mechanism and manifestations of this disorder.

\section{P-138}

\section{"Split hand" phenomenon in ulnar neuropathy}

\section{J.Zwicker, E. Pioro (Cleveland, USA)}

Background: The "split-hand" phenomenon (SHP) refers to predominant denervation of thenar muscles and first dorsal interosseus muscle (FDI) relative to hypothenar muscles. It has been reported to be suggestive of motor neuron disease. To examine the specificity of this finding, we studied the frequency of the SHP in ulnar neuropathy.

Methods: Studies of patients with pure ulnar neuropathies (UNs) at the Cleveland Clinic EMG laboratory between 1997 and 2001 were reviewed. Ulnar neuropathies resulting in low compound muscle action potential (CMAP) of the FDI, abductor digiti minimi $(\mathrm{ADM})$, or both, or fibrillations in one or more ulnar-innervated muscle, were analyzed retrospectively.

Results: Sixty-six patients with a total of $71 \mathrm{UNs}$ were identified. Normal CMAP responses were found in $17 \%$ (12/71). Low CMAPs occurred in only the FDI in I4\% (10/71), only the ADM in $10 \%$ (7/71), and in both FDI and ADM in 59\% (42/71). Three of the four cases with low FDI CMAP alone, and normal ulnar sensory nerve action potentials (SNAPs), were due to ulnar nerve lesions within the hand.

Conclusion: In our series, 5.6\% (4/71) of UNs resulted in a SHP with preserved ulnar SNAPs. Most of these resulted from an isolated lesion in the hand.

\section{NEURO-ONCOLOGY}

\section{P.139}

Inferior frontal lobe and chiasmal herniations resulting from dopamine agonist treatment of pituitary macroadenoma

\section{M.H. Bangash, D.B. Clarke, R.O. Holness (Halifar, Nova Scotia)}

Background: Dopamine agonists are widely used in the treatment of pituitary prolactinomas. We report a case of inferior mesial frontal lobe and chiasmal herniations into an enlarged sella, following successful medical treatment of a pituitary macroadenoma.

Method: A 71-year-old healthy man presented to medical attention with visual complaints. On examination, he was found to have bitemporal hemianopsia and had an endocrine work up that was remarkable for high prolactin. He was treated medically with a dopamine agonist (bromocriptine) and he responded well with return of near normal vision. After one year of treatment, routine visual field testing demonstrated a more profound asymptomatic bitemporal visual field defect. Neuroimaging by MRI was performed.

Results: MRI of the sella showed right mesial frontal lobe herniation inferiorly into an enlarged sella with associated chiasmal compression. The patient's vision stabilized (with significant bitemporal field loss) and he continued to be treated non-operatively.

Conclusions: We report a case where successful medical treatment of a large pituitary prolactinoma resulted in inferior frontal lobe and chiasmal herniations into an enlarged sella.

\section{P-140}

Intradural-intramedullary hemangioendothelioma of the spinal cord - a case report

A.Cenic, S. Abuzallouf, R. Wong, J. Provias, A.P. Mintz (Hamilton, Ontario)

Background: Hemangioendotheliomas (HE) are vascular neoplasms of intermediate or borderline malignant potential. They are composed of endothelial cells and occur in soft tissues, liver, 
lung and bone. They rarely involve the neuraxis. We report the first case in the literature of intradural-intramedullary HE of the spinal cord.

Methods: A 41 -year-old gentleman presented with low back pain, numbness and urinary retention. On MRl imaging, an irregular, lobular strongly contrast enhancing hemorrhagic mass was seen opposite to the T 12 vertebral body.

Results: The patient underwent a laminectomy from $\mathrm{T} 12-\mathrm{LI}$ and the tumour was resected. Postoperative radiotherapy (50.4 Gray in 28 fractions) was delivered to enhancing tissue seen on the postoperative images. The patient is doing well with residual numbness and mild left hip flexor weakness. His urinary and bowel symptoms resolved. There is no radiation toxicity and no residual disease on MRI at 27 months of radiologic and 32 months of clinical follow-up.

Conclusions: This case report describes a rare presentation of a HE located in the intradural-intramedullary location. Complete excision of $\mathrm{HE}$ is the treatment of choice. The role of adjuvant radiotherapy has not been clearly defined but may play a role in the management of this lesion.

\section{P-141}

\section{Descriptive epidemiology and waiting times for treatment of primary intracranial neoplasms in the Hamilton region}

\section{A. Cenic, J.Wu, H. Rhydderch, A.P. Mintz (Hamilton, Ontario)}

Background: The Hamilton regional cancer centre (HRCC) has a referral base of over two million people. Epidemiological data for a Canadian regional neuro-oncology program has not been previously reported. To guide management of this resource intensive population, descriptive statistics and waiting times are essential.

Methods: Beginning in 1999, prospective data was collected at the HRCC on adult patients with primary intracranial tumours. Descriptive statistics and treatment dates were obtained on each patient. Waiting times between surgery, HRCC consultation and onset of radiation were calculated.

Results: From 1999-2000 a total of 118 primary tumours were identified with $58 \%$ occurring in men. Mean age of presentation was 54.6 years. Similar histological distributions were seen in both sexes. Glioblastoma represented $55 \%$ of the tumours. Waiting times from date of surgery to HRCC consultation was 17 days. The time from the consultation to start of radiation was 44 days in 1999 , which decreased to 35 days in 2000 . Time from surgery to start of radiation was 46 days.

Conclusions: Histological, sex and age distributions were similar to previously reported North American studies. Waiting times from surgery to radiation therapy were over 6.5 weeks and represent significant treatment delay. Data collection is ongoing.

\section{P-142}

\section{Brainstem talc granuloma: a case report}

\section{Crevier, K. Reddy (Hamilton, Ontario)}

Background: Magnesium silicate (taIc) is a common, widely used substance and usually considered to be benign. It can unfortunately sometimes lead to granuloma formation. Those talcomas or talc granulomas have been described in most anatomic sites. To our knowledge, talcoma has been documented at the ventricular end of ventriculoperitoneal shunts, but never in the brainstem.

Methods: A 40-year-old man presented with a five-year history of progressive paraparesis and walking difficulty. The initial MRI showed arachnoiditis-type findings but a subsequent MRl revealed a cervicomedullary junction intra-axial tumor.

Results: The lesion was partially resected via a far lateral approach. To our great astonishment, pathological investigation demonstrated a foreign body granuloma including talc particles. We think this could be related to surgical glove powder since the patient had communicating hydrocephalus as a child requiring a ventriculoperitoneal shunt and multiple lumbar punctures.

Conclusion: Intramedullary talc granuloma is a very rare finding, but its occurrence must be kept in mind especially when the patient had a prior surgical procedure involving the CNS.

\section{P-143}

Comparison of imaging modalities for intracranial lesions and their utility in preoperative assessment

S. Gopinath, R.G. Beran, J. VanGelder, M. Sheridan, J.M.G. Chu, C. Chow, T. Sachinwalla (Liverpool, Australia)

Background: Intracranial space occupying lesions may be malignant, inflammatory or degenerative and further management depends on the underlying pathology. This pilot study was designed to compare and assess the utility of cerebral imaging as an adjunctive tool in their management.

Methods: Patients who presented to the Liverpool Hospital between March 1998 and September 2000, with features of intracranial lesions not requiring urgent excision, were selected. They were investigated with standard magnetic resonance imaging using a $1.5 \mathrm{~T}$ Phillips ACS-NT MRI, complemented by $\mathrm{H} 1$ spectroscopy (SVS/2) TS $1272 \mathrm{exc}$ ) and FDG CoPET using an ADAC Solus MCD dual-head gamma camera (128 matrix, 32 steps at $80 \mathrm{sec} / \mathrm{step} . z 00 \mathrm{~m} 2.19)$. The results were correlated with pathology from biopsied specimens.

Results: 20 patients were studied of whom 15 had neoplasia. PET imaging diagnosed 10 with tumours and all 5 non-tumours with sensitivity and specificity for malignancy being $67 \%$ and $50 \%$ respectively. Of the 18 studied with MRS, 12 were correctly identified as malignant providing sensitivity and specificity of $92 \%$ and $20 \%$ respectively.

Discussion: This study provides a qualitative assessment and comparison of two imaging techniques. The tests allow better planning and prognostication and may be employed to avoid surgery when confirmed by larger studies.

\section{P-144}

\section{The ratio of cranial to spinal tumors stratified by pathology}

\section{P. Muller J. Bilbao (Toronto, Ontario)}

Background: There is a considerable diversity of neurological tumors; however, patterns of age and localization emerge.

Method: We examined our neuro-oncologic database and found 390 adult spinal tumor patients among 3402 histologically confirmed neurosurgical tumors.

Results: The ratio of primary intracranial tumors to primary spinal tumors was $11 / 1$. The ratio of intracranial intradural tumors to 
spinal intradural tumors was $12 / 1$. The ratio of astrocytic tumors [all grades] was $68 / 1$. The ratio of intracranial to spinal meningioma was 10/1. The ratio of acoustic neuroma to spinal schwannoma was 1.5/1. The ratio of intracranial ependymoma to spinal ependymoma was $1 / 1$. Of the spinal tumors there were 166 secondary carcinomas with a male/female ratio of $1.22 / 1$ and a mean age of 56 years located almost exclusively in the extradural compartment [54\% of spinal tumor patients who under went surgery]. There were 224 primary spinal tumors distributed as follows: 113 intraduralextramedullary [29\% of total] and 53 intramedullary [14\%]. The ratio of intradural extramedullary to intramedullary tumors was 2.1/1.

Conclusions: Among gliomas the distribution of tumors in the brain and cord may be related to tissue mass and among meningiomas the distribution may be related to arachnoidal surface area or concentration of arachnoid cap cells.

\section{P-145}

Recurrence of a spinal meningioma at a second intradural spinal location

\section{P. Muller, T. Gray, J. Bilbao (Toronto, Ontario)}

Background: The recurrence of spinal intradural meningiomas after complete resection is less common than their intracranial counterparts. $19-37 \%$ of intracranial meningiomas recur after gross total resection; the rate in spinal meningiomas is $4-10 \%$.

Method: We report an individual who underwent a complete resection of a T4-T5 level meningioma who had a new T12-LI level meningioma 42 months postoperatively.

Case report: A 50-year-old female presented in June 1998 with a 6-week history of leg weakness, unsteadiness and cold dysesthesia. An MRI showed a well-defined $2-\mathrm{cm}$ enhancing intradural extramedullary mass. The lesion was completely resected along with its dural attachment. A complete neurological recovery occurred. A follow-up MRI scan 19 months postoperatively showed no evidence of tumor at T4 and a suspicious $5 \mathrm{~mm}$ enhancing lesion at T12-L1. At 41 months MRI showed enlargement to $1-\mathrm{cm}$. She complained of dysesthetic right leg pain. An uneventful resection was carried out.

Results: The original pathology was that of a transitional psammomatous meningioma. The second tumor was a transitional psammomatous meningioma with occasional mitotic figures and bone invasion but no anaplasia.

Conclusion: Although recurrent meningiomas usually recur at their original site, distant recurrence may occur with seeding or genetic disposition.

\section{P-146}

\section{Clinical exacerbation of MS following radiotherapy: a case} report

\section{Murphy, S. Hashimoto, D. Graeb, B. Thiessen, (Vancouver,} British Columbia)

Background: Radiation of the CNS in patients with demyelinating disease may have deleterious effects.

Methods: A case report of a 30-year-old woman with MS who developed an attack of demyelination two months following radiotherapy for a parotid malignancy.

Results: MRI demonstrated new hyperintense lesions that corresponded to both the localization of the patient's symptoms and to the area defined by the $50 \%$ isodose radiation field.

Conclusions: Radiation treatment likely triggered an MS exacerbation.

\section{P-147}

Paraneoplastic limbic encephalitis and profound language disturbance secondary to an occult atypical carcinoid tumour of the lung

Z. Rubin, R. Camicioli, M.S. Hussain, J. Kashmere, T. Roberts, A. Easton (Edmonton, Alberta)

Background: Paraneoplastic limbic encephalitis (PLE) is a rare complication of systemic malignancy. Presenting features of PLE often precede the discovery of an underlying neoplasm and may mimic various complications of cancer or neurologic disease.

Methods: Case report / literature review

Results: A 40-year-old woman presented with ataxia and generalized sensory symptoms. Complete laboratory investigations were negative. MRI showed increased signal intensity on T2weighted images in the left insula, right occipital, and posterior parietal lobes. CT chest, lung biopsy revealed atypical carcinoid tumour of the lung. The patient developed progressive memory loss and severe conduction aphasia, despite plasmapheresis and IVIg. Antibodies were negative (Anti-Hu, Anti-Yo, Anti-Ma, Anti-Ro). CSF was positive for protein 1433. The patient was readmitted with respiratory failure and apneic spells. Care was withdrawn and the patient died. Autopsy consistent with PLE.

Conclusions: This is the second reported case of carcinoid tumour associated with PLE. Our patient tested negative for common antibodies and did not respond dramatically to conventional therapies. Given the evidence for residual tumour on radioisotope scanning, complete tumour resection or chemotherapy should be considered in future. The patient deteriorated before this could be pursued. Conduction aphasia should be recognized as a component of PLE.

\section{P-148}

Petrous apex chondrosarcoma associated with Maffucci's syndrome: a case report and review of the literature

\section{J.Spears, J. Riva-Cambrin, D. Rowed (Toronto, Ontario)}

Background: Maffucci's syndrome is a rare clinical entity characterized by multiple enchondromas and cutaneous hemangiomas which, very rarely, can be associated with cartilagenous skull base tumors. We have encountered only ten previously reported cases of Maffucci's syndrome associated with skull base chondrosarcomas.

Methods: We report a 22 -year-old male who presented with a right abducens nerve palsy. Examination revealed that he also had multiple cutaneous hemangiomas and bony masses consistent with enchondromas involving the extremities. CT and MR imaging revealed a large right-sided basal skull mass with bony destruction of the petrous apex and clivus with tumor extending into both the posterior and middle cranial fossae. Biopsy confirmed a diagnosis of low grade sarcoma. The patient underwent gross total resection by a cranio-orbitozygomatic approach with reconstruction of the dural defect using a fat and fascia lata graft. Cranial nerve and 
somatosensory evoked potentials (SSEPs) were monitored intraoperatively.

Results: Postoperatively the patient did well, with transient incomplete III, V, VII and VIII nerve palsies. Serial postoperative MR imaging in follow-up will guide further adjuvant therapies including possible proton beam radiation.

Conclusions: Maffucci's syndrome is an extremely rare syndrome providing a diagnostic challenge when associated with skull base chondrosarcomas. We discuss the clinical features of this rare clinical entity and appropriate management strategies. A review of the literature will be presented.

\section{P.149}

\section{Development of a brain tumor in MS: case report}

\section{A. Traboulsee, C. Laule, C. Keogh, J. MaGuire, G. Redekop (Vancouver, British Columbia)}

Background: A woman with a nine year history of multiple sclerosis (MS) presented with anorexia and vomiting of three months duration. Although clinical deterioration is expected in most patients with MS due to relapses or secondary progression, routine MRI is not often done to determine if this is due to a secondary diagnosis. Lesions caused by MS rarely exceed $2 \mathrm{~cm}$ in diameter, although large tumefactive MS lesions have been reported.

Methods: Neurologic exam and gadolinium enhanced MRI and MR proton spectroscopy (MRS) were performed using a I.5T GE scanner prior to brain biopsy. The biopsy was processed for immunohistochemical studies and electron microscopy.

Results: MRI demonstrated a $4.6 \times 4.8 \mathrm{~cm}$ left frontal mass with mixed signal intensity, edema, significant mass effect and irregular ring enhancement with gadolinium. The NAA signal was absent in the lesion. Histopathology showed characteristics typical of a giant cell glioblastoma (grade IV astrocytoma).

Conclusion: MRI imaging and stereotactic brain biopsy are indicated for MS patients with an unexpected deterioration to identify a secondary diagnosis such as a neoplasm or infection. MRS is particularly useful in the investigation. The co-occurrence of malignant glioma with MS is rare, and it is uncertain whether such an association is purely co-incidental.

\section{$\mathbf{P} \mathbf{1 5 0}$}

Melanotransferrin, an Alzheimer's-associated protein, is a potential vector for the delivery of chemotherapeutic agents across the blood-brain barrier

S. Tsai, R. Gabathuler, G. Arthur, M.L. Kennard, J. Yang,

G. Laliberte, W. Schoorl, D. Karkan, (Vancouver, British

Columbia)

Background: Melanotransferrin ( $\mathrm{p} 97$ ), a membrane-bound homologue of transferrin, exists in minute quantities as a soluble form in serum. In Alzheimer's disease, its presence in amyloid plaques and elevated serum levels suggest its ability to traverse the blood-brain barrier (BBB).

Methods: To determine whether p97 crosses the BBB, iodine[125]-labelled recombinant soluble p97 was injected intravenously into $\mathrm{C} 57 \mathrm{BL} / 6$ mice and into athymic nude mice with intracranial $\mathrm{C} 6$ glioma, and its uptakes by the brain and tumours were monitored. To ascertain its transport mechanism, in vivo selfinhibition of iodinated versus unlabelled p 97 was studied. Finally, to assess its potential as a delivery vector, p97 was conjugated to adriamycin and the brain uptake was measured.

Results: p97 levels in the brain increased ten-fold during first 120 minutes, demonstrating its ability to cross the BBB. p97 significantly exceeded the control's (albumin) ability to cross the BBB, with greater amounts of $\mathrm{p} 97$ accumulating in intracranial tumours than the brain. Further, competitive inhibition occurred, signifying saturable receptors regulate its transport. Finally, p97adriamycin conjugates exhibited similar brain uptakes to $\mathrm{p} 97$ alone.

Conclusions: $\mathrm{p} 97$ crosses the $\mathrm{BBB}$ through receptor-mediated transcytosis, and selectively accumulates in intracranial tumours, reflecting its promising potential as a chemotherapeutic delivery vector to intracranial tumours.

\section{P-151}

Surgical and technical feasibility of 5-aminolevulinic acid (5ALA) based fluorescence guided resection of intracranial VX2 tumor in a rabbit model

A. Varma, A. Bogaards, V. Yang, L. Lilge, A. Lin, B.C. Wilson, P.J. Muller (Toronto, Ontario)

Background: Fluorescence guidance may help to identify tumor tissue that is difficult to detect using white light microsurgery. A 5ALA based fluorescence guidance technique is being developed to discriminate tumor from normal brain tissue. 5ALA does not fluoresce, but is metabolized into the strongly fluorescent protoporphyrin IX (PpIX) by tumor cells, through enzymes of the heme-biosynthesis pathway. Excited with blue light PpIX enits a distinct red fluorescence, which can be detected by a sensitive camera or spectrograph. To investigate the surgical potential and drug dose dependence of this technique we have developed an animal model incorporating our recently developed fluorescence imaging and spectroscopy system. Our objective is to describe the surgical and technical feasibility using this animal model.

Methods: Four SPF New Zealand White rabbits received 105 VX2 cells in $50 \mu \mathrm{l}$ PBS as implant in the cortex at a depth of $3 \mathrm{~mm}$. Fourteen days post implantation the animals received $20 \mathrm{mg} / \mathrm{kg}$ 5ALA i.v. Six hours later tumours with a diameter of $8-12 \mathrm{~mm}$ were resected using a standard operation microscope and additional fluorescence guidance. Fluorescence images and spectra were taken throughout the entire operation exciting the tissue with $2.8 \mathrm{~mW}$ blue light with a wavelength of $405 \mathrm{~nm}$. Immediately after tumour resection the animals were euthanased and the brains fixed for serial sectioning and histopathological examination, in order to estimate residual tumour volume.

Results: We were able to detect tumor and tumor margins using 5ALA induced fluorescence guidance and identified residual tumor after white light resection.

Conclusions: Further evaluation of fluorescence guided brain resection using this animal model comparing conventional resection with fluorescence guidance is underway. This investigation is supported in part by grant CA $43892 \mathrm{DHHS} / \mathrm{NIH} / \mathrm{NCI}$. 


\section{P-152}

\section{Surgical treatment of malignant glioma in the elderly}

\section{A. Weeks, S. Hentschel, B. Toyota (Vancouver, British Columbia)}

Background: There was a perceived increased incidence in the peri-operative complications in the elderly undergoing craniotomy for malignant glioma at our institution.

Methods: A retrospective study was performed comparing patients undergoing craniotomy for malignant glioma. Group I consisted of patients over 70 years $(n=19)$ while group II consisted of patients less than 70 years $(n=20)$ over a five year period.

Results: The mean age in group I was 75 years (range 70-81). The mean age of group II patients was 49 years (range 27-68). In group I, pathology demonstrated glioblastoma (GBM) in $86 \%$ of the cases, with $90 \%$ of patients undergoing partial resections. In group II, 53\% were GBM, and $62 \%$ underwent 'complete' resections. In group I, there was an average of 1.2 complications/patient with infection, worsening of pre-existing neurological symptoms, and transient mental status changes accounting for the majority, while in group II there was an average of 0.4 complications/patient with new neurological symptoms and mental status changes being the most common. Pre-operatively, the average Karnofsky performance scores were similar in both groups (74.5 for group I and 76.7 for group II). Postoperatively, however, the average change in Karnofsky scores were significantly different ( -15.5 for group I and -0.48 in group II, $\mathrm{p}<0.0 \mathrm{I}$ ).

Conclusion: Patients over 70 years of age have more peri-operative complications resulting in a significant decline in their Karnofsky scores postoperatively when compared with younger patients.

\section{SPINAL SURGERY}

\section{P.153}

Clinical efficacy and cost-effectiveness of spinal cord stimulation in failed back surgery patients in Atlantic Canada

\section{A.Y. Alemdar, I. Mendez (Halifax, Nova Scotia)}

Background: The objective was to examine the efficacy and costeffectiveness of spinal cord stimulation (SCS) for failed back surgery syndrome (FBSS) in Atlantic Canada.

Methods: Thirty-four patients entered the trial. Patient information was gathered pre-operatively. Implantation and followup data were also collected. Outcome measures included changes in medications, employment, and therapy satisfaction. Yearly costs were compared before and after implantation.

Results: Three patients were lost to follow-up before one year. Of the remaining patients: $12.9 \%$ - screened only; $9.7 \%$ - system removed before one year; $38.7 \%$ - regular stimulator use, continued medications; $29 \%$ - regular system use, no medications; $9.7 \%$ stopped using system after one year. Mean follow-up time in patients using system longer than one year was 34.6 months $(n=24) .26 \%$ of patients potentially able to return to work did so $(n=23)$.

SCS implantation cost $\$ 16,702.64$. With decreased yearly costs after implantation, SCS paid for itself in 6.5 years $(\mathrm{n}=31)$. In patients using the stimulator longer than one year SCS paid for itself in four years $(n=24)$. Best-case scenario was in patients who went back to work, where SCS paid for itself in 1.5 years $(n=6)$.
Conclusions: SCS is an efficacious and cost-effective procedure for the treatment of pain in selected FBSS patients.

\section{P-154}

\section{Posterior lumbar interbody fusion using femur}

K. Al-Musrea, B.Lo, Karrie Brennan, Eric Belanger, (Ottawa, Ontario)

Background: Posterior lumbar interbody fusion (PLIF) is an increasingly popular means to fuse the lumbar spine. By substituting the conventional cages with intra-operatively shaped femur allograft, we have been able to replicate the success rates of PLIF while drastically cutting out the costs of the cages.

Methods: A total of 12 patients who underwent lumbar interbody fusion between July 2000 and June 30 th, 2001 were included in this retrospective study. Follow-up visits occurred between three and 12 months. $15 \mathrm{~mm}$ rings of femur, which have been cut and shaped to the appropriate height and length intra-operatively, are ready within a matter of minutes to be used as a biological spacer in the intervertebral space.

Results: The preoperative to postoperative VAS changes were as follows: VAS back pain improvement of 2.8 points and leg improvement of 5.6 points. There has been no instrumentation failure to this point.

Conclusions: Custom-made intra-operatively shaped femur allograft used for PLIF, is an acceptable alternative to the more expensive cage. The femur ring is easy to shape to fit perfectly in the interbody space.

\section{P-155}

Long-term follow-up of selective dorsal rhizotomy: does multilevel laminotomy influence the risk of spinal deformity?

\section{J. Hall, J-P. Farmer, A.M. O'Gorman (Montreal, Quebec)}

Background: One hundred patients have undergone selective dorsal rhizotomy (SDR) for relief of spasticity at the Montreal Children's Hospital. This procedure involves surgically exposing and sectioning the lumbosacral roots and requires a significant bony opening. Traditionally, laminectomies with no laminoplasty were performed from L2 to S1. To reduce the possibility of instability and future spinal deformity, the technique of laminotomy with total reconstruction of the posterior spinal arch has been proposed. We have used this latter procedure in all of our patients. It is hypothesized that laminotomy results in a lower incidence of spinal deformity than that associated with multi-level laminectomies.

Methods: Pre- and postoperative radiographs have been examined for spinal deformity (scoliosis, kyphosis, lordosis) using Cobb's angles with a follow-up period of up to ten years. This data will be presented and compared to that reported in the literature following laminectomies for SDR.

Results/Conclusion: Laminotomy with laminoplasty is associated with a low incidence of spinal deformity in the available follow-up period of our patients. While continued surveillance into adulthood is required to confirm the stability of this finding, this procedure appears to convey an advantage over laminectomy and should be recommended for pediatric spinal surgery. 
The relationships between social support, life stress, and quality of life following spinal decompression surgery

A.W. Laxton, R.G. Perrin, (Toronto, Ontario)

Background: The purpose of this study was to examine how social support and life stress relate to patients' quality of life following spinal decompression surgery.

Methods: Nineteen patients with degenerative spine disease who had undergone spinal decompression surgery within the past 14 months filled out social support, life stress, and quality of life questionnaires. Correlational techniques were used to assess the relationships among the variables.

Results: The correlations between social support scores and health-related and non-health-related quality of life scores were $r=$ $0.72, p=.001$, and $r=0.50, p=.028$, respectively. The correlations between life stress scores and health-related and non-health-related quality of life scores were $r=-0.83, p<.001$, and $r=-0.72, p=.001$, respectively.

Conclusions: Patients with degenerative spine disease experiencing more social support and less life stress report greater satisfaction in health-related and non-health-related quality of life following spinal decompression surgery than those with less social support and more life stress. Addressing social support and life stress in the management of patients with degenerative spine disease could help to improve patients' satisfaction with their medical outcome and general quality of life following spinal decompression surgery.

\section{P-157}

\section{Primary extraosseous spinal epidural Ewing's sarcoma}

\section{A. Modha, D Gandhi, M. Goyal, E. Belanger (Ottawa, Ontario)}

Introduction: Primary extraosseous spinal epidural Ewing's sarcoma is a rare malignant disease that can cause cord compression.

Methods: A 33-year-old healthy male presented with myelopathy due to thoracic spinal cord compression. He had a sub-acute onset of upper back pain and progressive paraparesis. MRI scan revealed an epidural lesion extending from T4 to T7. A laminectomy was done at the appropriate levels and the cord was decompressed.

Results: Pathological analysis confirmed the presence of Ewing's sarcoma. Postoperatively, the patient's strength improved and he became ambulatory. A metastatic work up was negative. After receiving chemotherapy and radiation therapy, he has been disease free for nine months.

Discussion: The case study includes a review of the literature and details of the pathological and radiological findings. So far, only 17 cases of this disease have been reported. In several cases, a herniated intervertebral disc was suspected as the cause of neurological compromise. This misdiagnosis may lead to a delay in surgery and treatment. Good imaging techniques and a strong index of suspicion is necessary to rule out this very malignant disease. Inadequate data exist about the efficacy of chemotherapy and radiation and little is known about long-term survival. However, complete resection can lead to improved survival and prognosis.
Cervical open door laminoplasty: the mini plate and key hole technique

\author{
S. Paquette, Fawaz Sidiqi, Karrie Brennan, Eric Belanger, \\ (Ottawa, Ontario)
}

Background: At the Ottawa Hospital, an increasing proportion of patients suffering from cervical myelopathy are being treated with our modified version of the cervical open door laminoplasty. It is believed that this procedure may reduce the rate of post laminectomy kyphosis.

Methods: The charts of 12 patients were reviewed for presentation, pre- and postop radiological evolution and outcome. Postoperative follow-up extended from three months to 18 months. The surgical technique used was a modification of Hirabayashi's technique. A unilateral opening is performed followed by a greenstick fracture contralaterally. The "door" is kept open by the use of a mini-plate. Foraminotomy is achieved, when needed, by the keyhole technique.

Results: Twelve patients were operated on for cervical myelopathy. Radiological decompression was achieved in all cases. Complications included one wound infection and one transient C5 palsy. All 12 patients showed a certain degree of improvement.

Conclusions: Cervical open door laminoplasty using a mini-plate is an effective way to decompress the cervical cord. The complication rate compares favorably with anterior decompression. The late post laminectomy kyphosis may be avoided with laminoplasty.

\section{P-159}

Feasibility of transplantation of subependymal stem cells into mouse spinal cord after acute and chronic spinal cord injury

\section{E.C.Tsai, L. Lee, C. Morshead, A.J. Mothe, D. van der Kooy,}

\section{C.H. Tator (Toronto, Ontario)}

Background: Stem cells from clonally derived neurospheres from the subependyma of the adult mouse forebrain hold promise as part of a therapeutic strategy for spinal cord injury (SCI). Because of high mortality with a T3-4 injury level, we examined the feasibility of subependymal stem cell transplantation at a T8 injury level after both acute and chronic SCI.

Methods: Female CD1 mice received a moderate T8 clip compression injury. At 3 (acute) or 21 (chronic) days post injury, animals were stereotactically injected at the injury site with subependymal stem cells $(n=18)$ or fibroblasts $(n=18)$ derived from ROSA26 mice containing the $\beta$-galactosidase construct, or artificial cerebrospinal fluid $(n=18)$. The BBB locomotor functional assessment was performed weekly.

Results: Functional improvement was found only in the group with stem cell implantation after chronic injury $(\mathrm{p}<0.05)$. In both acute and chronic SCI animals, clusters of B-galactosidase positive cells were found at the injury site. The overall survival rate was $78 \%$.

Conclusions: Transplanted subependymal cells are viable postinjection after acute and chronic $\mathrm{SCl}$, and functional neurological improvement was seen post-injection of stem cells after chronic SCI. Use of subependymal stem cells suggests a novel therapeutic strategy which may hold promise for those with chronic, traumatic $\mathrm{SCl}$. 


\section{Repair of spinal cord injury using a novel synthetic graft}

E.C.Tsai, P.D. Dalton, M.S. Shoichet, R.L. van Bendegem, C.H. Tator, (Toronto, Ontario)

Introduction: As synthetic grafts facilitate only limited axonal regeneration after spinal cord injury, we examined the biocompatibility of a novel tubular device, composed of a rigid or malleable formulation of poly (2-hydroxyethyl methacrylate) (PHEMA), that can be bioengineered to provide improved haptotactic and chemotactic cues of regeneration.

Methods: Adult Sprague Dawley rats $(n=16)$, with complete spinal cord transection at T8, had their cord stumps inserted into a rigid or malleable PHEMA tube. Fibrin glue was applied to the cordtube interface, and Gortex was used for duraplasty. Controls $(n=3)$ underwent cord transection alone. Animals underwent axonal tracing and immunohistochemistry, and were examined after 2,4 , and 8 weeks.

Results: There was ingrowth of neural tissue through the tubes as early as two weeks. Tissue between the stumps was seen more consistently with the rigid tubes, and contained neurofilament stained axons. With both tube types, there significantly less scarring at the tube-dura interface compared to at the Gortex interface, and calcium deposition on the exterior tube surface occurred.

Conclusions: Rigid PHEMA tubes may help promote continuity of neural tissue in transected spinal cord, and further work modifying the contents of the tube to promote enhanced regeneration is ongoing.

\section{General Neurosurgery}

\section{P-161}

\section{A history of neurosurgical research at Dalhousie University, Halifax, Nova Scotia}

\section{A.Y. Alemdar, S.D. Christie, I. Mendez (Halifax, Nova Scotia)}

When Dr. W.D. Stevenson established neurosurgery in Nova Scotia in 1948, he did not have time to devote to research because of a large clinical caseload. However, once joined by a second neurosurgeon in 1955, investigations were begun on an animal model of hydrocephalus. Such experimentation was short-lived however, due to lack of facilities and the clinical demands. In spite of this, research was still encouraged and the Department served as an investigational site for many clinical trials. The invention of the Halifax Clamp in the early 1980s was a significant contribution to the field of neurosurgery. The clamp continues to be used today as an option for posterior internal fixation. Basic science research underwent a resurgence in the 1990s with the arrival of $\mathrm{PhD}$-trained neurosurgeons. Since then, neurosurgery residents have also become more involved in research, undergoing dedicated research rotations and post-graduate research training. The Division is still very involved in numerous clinical trials and has the country's only clinical program in neural transplantation. The future looks promising with the creation of a Brain Repair Center, which will enable further growth in neurosurgical research in Halifax.

\section{Disseminated tuberculomas of the brain}

\section{A. Alkhani, F. Al-Otaibi, E.J. Cupler, B. Lach, (Riyadh, Saudi} Arabia).

Background: In some areas of the world, tuberculosis (TB) of the central nervous system is quite common and always considered in the differential diagnosis of enhancing intra-axial lesions. TB of the brain can present in many different clinical and radiological patterns. Disseminated or miliary TB of the brain is a relatively rare entity with peculiar clinical and radiological features.

Method: We describe the case of a 25-year-old immunocompetent female who presented with one month history of progressive headache and unsteadiness of her gait. MRI of the brain reveals multiple small enhancing lesions measuring a few millimeters in both supra- and infra-tentorial compartments. The lesions (tuberculomas) were not visible on an enhancing CT study of the brain. There was no evidence of TB outside the CNS.

Results: The patient was diagnosed to have multiple caseating granulomas using an open biopsy. She improved clinically as well as radiologically after starting anti-TB therapy. Radiological and pathological studies are presented with a literature review of the topic.

Conclusion: Disseminated tuberculomas of the brain is a rare and unique clinical and radiological entity even with no other systemic involvement, it should be considered in the differential diagnosis of multiple small brain lesions.

\section{P-163}

The treatment of trigeminal neuralgia in patients with multiple sclerosis utilizing percutaneous rhizotomy: a clinical study in the British Columbian population

\section{C.Berk, C.R. Honey (Vancouver, British Columbia)}

Background: Trigeminal neuralgia (TN) has a $2 \%$ incidence among patients with multiple sclerosis (MS). This cohort presents a series of management challenges including poor tolerance of antineuralgic medications and occasional bilateral presentation. The review studied the results of percutaneous rhizotomy for this condition.

Methods: Patient records were reviewed retrospectively between the years 1996-2000. The data regarding age, sex, duration of primary disease and pain, response to medical treatment, pain distribution and outcome during the follow-up were evaluated.

Results: There were 86 patients treated with percutaneous rhizotomy for TN of whom thirteen had MS. The average age of onset for MS was 41 and for TN was 49 years. The lifetime average number of procedures for this cohort was two. Complete pain relief without any medication was achieved in $81 \%$ and with continued medications in the remaining $19 \%$ of the patients during a mean follow-up period of 26 months. There were no complications related to the procedure.

Conclusion: Percutaneous rhizotomy is a safe and effective method for the treatment of TN in patients with MS. The unique susceptibility of this cohort to the side effects of anti-neuralgic medications argues for an earlier consideration of rhizotomy than for those without MS. 


\section{P.164}

Quality of Life Questionnaire for Cushing's disease

M.D. Cusimano, A. Marchie, F. Salehi, H.S. Smyth, (Toronto,

Ontario

Background: Patients with pathologically-verified Cushing's disease (CD) experience a range of symptoms. Understanding CD's impact on quality of life (QOL) is important in management. Our aim is to develop and validate a health-related quality of life questionnaire (QU) specifically for these patients.

Methods: Focus group interviews and literature review were used to develop a QU with 177 items. Item reduction was iterative and involved content analysis, dual scaling, and ranking of the items' impact. A refined QU with 57 items was generated and mailed with the SF-36, FACT-Br, and Karnofsky Scale (KPS) to CD patients. Validity coefficients were calculated between subscale scores of the instruments. QU scores in the extreme groups were compared by ttest to assess known group validity. Test-retest reliability was determined by the Pearson coefficient and a t-test between two sets of scores completed 7-9 days apart.

Results: Forty patients completed the refined QU. The emotional, cognitive, and physical domains were more affected by $C D$ than the medical and social ones. Validity coefficients with the SF-36 and FACT-Br were: social function $(0.21,0.21)$, emotional well-being $(0.61,0.34)$, total score $(0.58,0.68)$; and with KPS, total score $(0.14)$. Extreme groups $(n=20)$ were significantly different $(99.5$, $13.4 ; p<0.00016)$. Test-retest reliability $(n=31)$ was 0.86 . Scores $7-$ 9 days apart were not significantly different $(p=0.42)$. Seventy per cent of patients thought the QU captured an accurate sense of their QOL.

Conclusions: A QOL QU was developed for patients with CD, and was shown to have good evidence of validity and reliability. This evidence is the basis for further psychometric testing that is being carried out in the United Kingdom.

\section{P-165}

\section{What is an RNFA? Do you need one in your practice?}

\section{S.Ilton, J. Wells (Hamilton, Ontario)}

Background: We found several "opportunities for improvement" at the neurosurgical centre where we work. Operating room (OR) resources were constrained. Surgical assistants were not always available. Educational activities were threatened. Imperfect scheduling wasted valuable OR hours. Tracking of waiting lists and outcomes was incomplete. Research and quality assurance activities were sporadic. Neurosurgeons tended to feel over - worked in their clinical practices.

Method: A Registered Nurse First Assistant (RNFA) was introduced to our neurosurgical service in March 2001. In addition to first assisting in neurosurgical operations, she helped to track surgical patient information, create changes to improve OR use, assist in clinics, and educate patients and learners.

Results: Important improvements occurred. Procedures were performed more efficiently. Patients reported reduced anxiety and increased satisfaction. OR utilization became more efficient. A perioperative database tracks information pertinent to efficiency and outcome measurement. Learners welcomed training experiences with an expert nurse. With an RNFA helping in the clinics, the neurosurgeon's time in clinics decreased while productivity there increased.

Conclusion: Collaborative work with a neurosurgical RNFA can improve patient care, perioperative efficiency, medical education, quality measurement, research output, and the neurosurgeon's quality of work life.

\section{P-166}

Malignant peripheral nerve sheath tumor: a surgical approach L. Jacques, T. Del Duca (Montreal, Quebec)

Background: Peripheral nerve tumors are rare entities, usually consisting of benign lesions such as schwannoma or neurofibroma. Surgical resection is favored in the presence of a painful, enlarging mass causing neurological deficit. Surgery is also important for diagnosis.

Methods: Three cases of malignant peripheral nerve sheath tumors (MPNST) associated with NF-1 will be presented. Two patients did not have a positive family history. The histological findings were compatible with MPNST. Thorough investigations including full metastatic work-up is essential.

Resu/ts: One patient required an amputation six months following initial diagnosis, despite negative surgical margins. The remaining two patients show no evidence of recurrence at two-year follow-up.

Conclusions: MPNST is a very rare condition where the literature is very scattered. There is no evidence that adjuvant therapy or even an amputation improve the survival rate. Negative surgical margins influence recurrence rates. These patients frequently present with distant visceral metastasis, therefore a closer follow-up is essential.

\section{P-167}

Myositis ossificans causing brachial plexopathy

\section{Jacques, T. Del Duca (Montreal, Quebec)}

Background: Myositis ossificans is a rare condition that results from intramuscular proliferation of fibroblast and osteoblast deposition of bone and cartilage.

Methods: A 56-year-old woman presents with an enlarging supraand infraclavicular mass that resulted in a left flail arm and a painful hand.

Results: She underivent a combined supra- and infraclavicular approach and exploration of her left brachial plexus. The histological findings confirmed the diagnosis of myositis ossificans. Nine months after surgery, the patient recovered shoulder function, with significant improvement of hand function and pain status.

Conclusion: To our knowledge, this is only the second reported case of myositis ossificans resulting in a brachial plexopathy. Peripheral neuropathy involving the radial, median, or sciatic nerve has been described but is very rare. The surgical approach is warranted for diagnosis and if possible, reversal of neurological deficit. 


\section{Supratentorial neurenteric cysts: case report and review of the literature}

\section{E. Kachur, L-C. Ang, J.F. Megyesi (London, Ontario)}

Background: Neurenteric cysts are congenital abnormalities usually found within the mediastinum, but can occur in the central nervous system. They are rare congenital cysts with histological features that indicate an endodermal origin. Central nervous system neurenteric cysts are most commonly found ventral to the spinal cord at the level of the cervical or upper thoracic spine. Intracranial neurenteric cysts usually occur in the posterior fossa. Supratentorial neurenteric cysts are very rare.

Methods: We report the case of a 35-year-old female who presented with progressive headaches and seizure-like episodes. Magnetic resonance imaging showed a $4 \times 4 \times 4 \mathrm{~cm}$ cystic lesion within the parenchyma of the right frontal lobe. This was successfully excised. Pathologic examination revealed a neurenteric cyst. A medline search of the literature was performed to determine the incidence of neurenteric cysts located in this part of the central nervous system.

Results: Nine previous reports of supratentorial neurenteric cysts were found. Cyst locations included suprasellar, orbital apex and intraventricular. None identified an entirely intraparenchymal supratentorial neurenteric cyst that had been pathologically confirmed. A comparison chart will be presented.

Conclusions: To our knowledge, this is the first case of a supratentorial neurenteric cyst, pathologically confirmed, that has been surgically removed from a patient.

\section{P-169}

\section{Propionibacterium acnes infections in neurosurgery: a case} series

\section{Kelly R. Griebel, S. Sanche (Saskatoon, Saskatchewan)}

Background: Propionibacterium acnes ( $P$. Acnes) is a relatively avirulent organism that is part of the skin normal flora. Most patient isolates are considered contaminants, but in a small subset of patients $P$. Acnes can cause significant disease following surgery. We analyzed the occurrence of $P$. Acnes infections after neurosurgical procedures.

Methods: A microbiology laboratory database was searched over a five-year period at a regional neurosurgical centre. 141 cultures were positive for $P$. Acnes. A retrospective chart review identified six patients with $P$. Acnes infections following neurosurgical procedures.

Results: All patients had undergone a previous craniotomy. All patients presented with scalp swelling and three patients had purulent discharge. Symptoms occurred in five out of six patients approximately two months after the initial surgery. Symptoms ranged from two months to three years. The bone flap removal was the initial treatment. Variable types of intravenous antibiotics were used. The length of antibiotic treatment ranged from three weeks to four months. No recurrent infection was seen in five patients. One patient had recurrent infection after cranioplasty.

Conclusions: $P$. Acnes is a rare but important cause of neurosurgical infections after craniotomy. Removal of the bone flap and foreign body along with adequate antibiotic coverage helps reduce recurrence.
Symptomatic intradural extramedullary noncommunicating arachnoid cyst: report of two cases with review of the literature

\section{S. Malik, K. Kumar (Regina, Saskatchewan)}

Background: The vast majority of patients harbouring arachnoidal cysts are asymptomatic. Noncommunicating intradural extramedullary arachnoid cysts are more rare relative to the communicating intradural extramedullary cysts. Noncommunicating intradural extramedullary arachnoid cysts are a very rare cause of spinal cord compression. Rarely, these cysts can present with bizarre symptoms like angina.

Methods: We present two rare cases of intradural extramedullary non communicating arachnoid cysts. The first case caused progressive paraparesis in a 75-year-old female. The second case, a 40-year-old male, presented with signs and symptoms of angina with mild spasticity in the lower limbs. In both cases, magnetic resonance imaging revealed compression of the spinal cord as the etiology of presentation. The first patient being reported here, is the second oldest patient in the literature with an idiopathic spinal arachnoid cyst. The second case being reported is due to its unusual presentation which has not been reported in the literature.

Results: Both of the cases underwent surgical excision of the arachnoid cyst with resolution of symptoms and reversal of neurological deficit.

Conclusions: Our experience indicates a complete recovery is possible after surgical excision even when the symptoms are present for a long duration in the elderly.

\section{P-171}

Intrasellar chordoma mimicking pituitary adenoma: case report and review of literature

F. Maroun, L. McDonald, T. Smith, G. Murray, J. Jacob, P. Bartlett, G. O'Brien, A. Goodridge (St. John's, Newfoundland)

Background: Intrasellar chordoma is rare; it can mimic pituitary adenoma.

Methods: A 33-year-old man presented with a one week history of neck stiffness, several acute and worsening episodes of neck pain radiating to vertex and orbit bilaterally, diplopia and photophobia. Endocrinological and radiological investigations suggested a large nonfunctioning intrasellar adenoma. Total removal of a friable, relatively vascular, intrasellar lesion was achieved through the transphenoidal route. Histopathologic diagnosis was chordoma. At six month follow-up there was no evidence of recurrence. Local radiation is contemplated.

Conclusions: Intrasellar chordoma can mimic pituitary adenoma and consequent risk of suboptimal therapeutic intervention.

\section{P-172}

\section{Dramatic response of pituitary macroadenoma to bromocriptine therapy}

Scott Paquette, Charles B. Agbi, E. Keely, D. Zachon (Ottawa, Ontario)

We present a case of a 42-year-old male with a very large prolactin secreting macroadenoma who presented with evidence of 
raised intracranial pressure, bitemporal hemianopsia and a third nerve palsy. The patient's presenting serum prolactin level was over 14,000 micrograms/L while his other pituitary hormone levels were within normal limits. MRI studies demonstrated a large invasive macroadenoma extending beyond the pituitary fossa and compressing the optic chiasm. The patient was treated with oral bromocriptine and had a dramatic reduction in symptoms within days. There was a complete reversal of his third nerve palsy and normalization of his vision within a few days. The case demonstrates how a very large macroadenoma producing symptoms from mass effect can be treated medically and thus avoiding surgery. Few cases of such large macroadenomas managed medically alone have been reported in the literature.

\section{P-173}

A pilot study comparing traditional to image-guided localization of brain tumors in the operating theatre

\section{Spivak, M. Kelly, F. Pirouzmand (Saskatoon, Saskatchewan)}

Background: The use of image-guided neurosurgery has expanded. It allows the improved localization of anatomical lesions within the cranial vault. Although it has become the standard of practice in many centres, there are relatively few studies that compare it to traditional methods. This study prospectively compares traditional to image-guided localization of cranial lesions.

Methods: A prospective non-randomized study is underway. The neurosurgeon will draw out the projected image of the lesion on the skin using standard CT or MRI images to plan the procedure. The image-guided system (IGS) will similarly be used to plan the procedure. The two localizations will then be compared.

Results: Five patients were analyzed. Traditional localization of small lesions $(n=2)$, with a diameter of $<3 \mathrm{~cm}$ was erroneous by 2.0 $\mathrm{cm}$ in the anterior-posterior direction and $3.5 \mathrm{~cm}$ in medial-lateral direction. In large lesions $(\mathrm{n}=3)$ with a diameter $>3 \mathrm{~cm}$, traditional localization was off $2.5 \mathrm{~cm}$ in the anterior-posterior direction and 2.8 $\mathrm{cm}$ in the medial-lateral direction.

Conclusions: Although our sample size is small, we hope to confirm with out current prospective study that IGS improves localization of lesions compared to traditional methods. This would reflect on incision and craniotomy size.

\section{P-174}

\section{A resident's electronic survival guide for the neurosciences}

\section{Taylor, S. Crocker, J. Wells (Hamilton, Ontario)}

Background: Electronic access to information is being favoured by medical learners. As part of this change, we developed the internet Survival Guide for the Neurosciences at McMaster University in Hamilton

Methods: The Guide evolved from clinical scenarios which arose repeatedly and which generated similar questions among residents who trained at our centre. It was created as an on-site tool to aid in learning on the job and to facilitate practice for non-experts when immediate support is compromised, such as when working on-call or in remote clinical settings. Its content has been developed by residents and reviewed by attending physicians.

Results: It informs, calculates, and illustrates using images, videos and informatics as well as text. There are many links to related electronic and printed information sources. The electronic Guide resembles printed manuals which have been used by generations of residents in order to improve both their competence and their learning while engaged in clinical service.

Conclusions: The electronic Resident's Survival Guide for the Neurosciences provides information that is concise, readily accessible, up to date, and relevant to recurring clinical situations. The Guide is an "open concept." It welcomes new entries and thus encourages electronic publishing and information sharing by residents.

\section{P-175}

Covered stent obliteration of a large aneurysm associated with a persistent trigeminal artery

\section{R. Vandorpe, D.B. Clarke, W. Maloney, R. Berry (Halifax, Nova Scotia)}

Background: Aneurysms at the site of origin of a persistent trigeminal artery are uncommon. Endovascular occlusion of intracranial aneurysms is a novel technique which is limited currently to the proximal, more easily accessible segments, of internal carotid artery.

Methods: A 4l-year-old man presented to neurosurgical attention with new onset of left facial and head pain during the previous three weeks. On admission, the patient's neurological exanination was normal with the exception of decreased sensation to light touch and pinprick over the left face in the VI distribution. MRI and cerebral angiography demonstrated a large aneurysm originating from a persistent left trigeminal artery and extending into the left cavernous sinus. There was no evidence of hemorrhage. Endovascular treatment involved placing a polytetrafluoroethylene (PTFE) covered stent to occlude the origins of the trigeminal artery and aneurysm.

Results: Complete obliteration of the aneurysm was achieved with preservation of the ICA lumen. The patient's headaches resolved within one week of treatment.

Conclusion: Endovascular placement of a PTFE covered stent can be used to successfully obliterate a persistent trigeminal artery aneurysm. It is anticipated that further advances in stent design will expand the role of this technology in the treatment of intracranial aneurysms.

\section{Trauma}

\section{P-176}

Rapid onset of severe cerebral oedema in infantile traumatic brain injury evident on CT by six hours

\section{K.M. Barlow, K. Poskitt, P. Steinbok (Vancouver, British Columbia)}

Background: When asked to estimate the time of an inflicted head injury, the presence of severe cerebral oedema on computed tomography is often thought to indicate that injury occurred at least 24 hours earlier. In accidental traumatic brain injury, the time of injury is usually well-documented. In the published literature there are only rare reports of cerebral oedema on CT within 24 hours of traumatic brain injury in children, and only four cases where the children are less than two years of age. The clinical details and neuroimaging of such children are not well-documented. 
We report a fatal case of accidental traumatic brain injury in a 12 month-old boy. His initial Glasgow Coma Scale was 6 . There was no evidence of hypoxia or hypotension. Computed tomography performed six hours after the injury demonstrated severe cerebral oedema. Cerebral blood flow estimated by Xenon-enhanced CT measured $0-1 \mathrm{ml} / 100 \mathrm{~g}$ of tissue/minute throughout both cerebral hemispheres. Eighteen hours after the injury he fulfulled criteria for brain cleath.

Conclusion: This case demonstrates that caution must be used when using the presence of severe cerebral oedema to determine the timing of traumatic brain injury, this may have major implications in the timing of non-accidental trauma.

P-177

\section{Changing demographics in the spinal cord injury population of} Nova Scotia

S. Christie, C. Short (Halifax, Nova Scotia)

Background: Although spinal cord injury (SCI) demographics are well-described in the medical literature, the majority of this data is U.S. based. Canada represents a distinct population and extrapolations are not always generalizable. Nova Scotia, a population of approximately one million, has the unique advantage of one centre managing all of the SCIs that occur within the province.

Methods: We conducted a review of SCI patient demographics in Nova Scotia from 1975 to 1999. Data was obtained from the Canadian Paraplegia Association database. Analysis was restricted to the Nova Scotia population.

Results: The overall incidence of $\mathrm{SCl}$ has been stable, at 15.7 cases per year, which is below the U.S. means of 25-35 per million people. Furthermore, compared to U.S. data, our patients were older and there was a paucity of violent causes. The male to female ratio was $4: 1$. The number of incomplete injuries and paraplegics have risen relative to complete injuries and tetraplegics, respectively.

Conclusions: The demographics of the SCI population in Nova Scotia are changing and reflect demographics that are different from the U.S. This illustrates the need to establish Canadian specific prospective databases for monitoring changes and evaluating the impact of new medical and surgical therapies.

\section{P-178}

Role of apoptosis and death receptors after human spinal cord injury: implication for the development of novel neuroprotective strategies

W.B. Jacobs, M.G. Fehlings (Toronto, Ontario)

Background: Despite recent advances, current therapies for spinal cord injury (SCI) are ineffective. Novel neuroprotective agents are needed. Animal experiments have identified delayed apoptosis after SCI. Our laboratory has implicated the death receptors, Fas and p75NTR, as mediators of post-SCl apoptosis in experimental models. To determine if death receptor mediated apoptosis is relevant to human SCI, we have examined molecular mechanisms of cell death in acutely injured human spinal cord specimens.

Methods: Eight cases ( 2 females $/ 6$ males, mean age 53 years) with $\mathrm{SCl}$ to death interval of two weeks to two years were obtained from the Division of Neuropathology, Toronto Western Hospital.
Using hematoxylin and eosin/luxol fast blue staining, the morphology of injury epicenter, rostral and caudal sections was assessed by light microscopy. Apoptotic cells were identified via TUNEL staining and activated caspase-3 immunohistochemistry. Death receptor expression was determined using stains to Fas and p75NTR antibodies.

Results: An increase in TUNEL positive cells was noted as compared with age-matched controls. Caspase- 3 activation was present after SCI. Fas and p75NTR are also expressed in postmortem SCl specimens.

Conclusions: Our findings suggest that death receptor mediated apoptosis occurs after human $\mathrm{SCI}$ and that this mechanism is a clinically relevant target for neuroprotective strategies.

\section{P-179}

\section{Administration of pro-cysteine compound decreases the number of activated macrophages/microglia following spinal cord injury}

\section{H. Kamencic, M. Kelly, A. Damant, B. Juurlink, R. Griebel, (Saskatoon, Saskatchewan)}

Background: Decreasing oxidative stress by maintenance of tissue glutathione following spinal cord injury will decrease inflammatory responses by decreasing extravasation of monocytes as well as the activation of microglia.

Methods: An extradural aneurysm clip with calibrated force of $50 \mathrm{~g}$ was applied to $\mathrm{T} 6$ rat spinal cord followed by intraperitoneal administration of L-2-oxo-thiazolidine-4-carboxylate (OTC) or saline vehicle. The activated macrophages/microglia were counted by immunohistochemistry using ED-1 antibody.

Results: ED-1 positive cells expressed phagocytic morphology were present in high number in the ventral and dorsal white matter at the site of injury with fewer ED- 1 positive cells in gray matter at the site of injury. We found a large accumulation of ED-1 positive cells extradurally. Administration of OTC decreased the number $(\sim 40 \%)$ of activated macrophages/microglia at the site of injury as well as rostral and caudal to the site of injury.

Conclusion: Spinal cord injury, within the first 24 hours, is complicated by inflammatory mechanisms, including the influx of monocytes/macrophages, as well as the activation of resident spinal microglia and astrocytes. Administration of OTC results in decrease in activation of microglia and/or extravasation of monocytes and prevents much of the secondary damage following spinal cord injury.

\section{P-180}

\section{Delayed removal of penetrating nail from midbrain interpeduncular cistern}

\section{E. Roger, M. Maleki, G. Mohr, (Montreal, Quebec)}

Background: Various cases of penetrating foreign bodies have been documented in the literature, dating as far back as Phineas Gage. These objects are usually removed unless the clinical status of the patient dictates otherwise. Very little information is published on the long-term risk of not removing an intracranial foreign body.

Methods: We will present a case of delayed removal of a penetrating nail, 11 months after the initial trauma. The pertinent literature will be reviewed. 
Results: A 40-year-old man presented to us 11 months after sustaining a trauma from a penetrating nail through the right temporal bone, causing bilateral IIIrd cranial nerve palsy and right posterior cerebral artery vasospasm. The initially planned conservative management was changed at the insistent request of the patient and his family. Surgical removal was made via a right temporal craniotomy and the nail was removed uneventfully after dissecting arachnoid adhesions and simple pulling. Eight months later, there was already a sensible improvement in the bilateral ptosis.

Conclusion: Removal of retained intracranial metal fragments is indicated in selected cases since long-term oxidation may produce meningeal fibrosis from chronic foreign body reaction, as well as delayed infection.

\section{P-181}

Therapeutic hypothermia in severe head injury: two year retrospective analysis

E. Roger, D. Klironomos, J. Duplantie, M. Feyz, MJ.M. English, M. Maleki, K.M. Johnston, (Montreal, Quebec)

Background: Many Class III experimental studies have demonstrated beneficial physiological effects of hypothermia in severe head injuries. Several Class I prospective randomized control trials have recently been performed in the context of severe head injury. Clifton (1993) and Marion (I997) showed improved outcome when prophylactic hypothermia was induced upon admission, regardless of the intracranial pressure. Shiozaki (1993) and Jiang (2000) studied the use of hypothermia for ICP refractory to standard management. They demonstrated reduced mortality and improved functional outcome. A phase III (class I) multicenter trial (Clifton, 2001) recently found that prophylactic hypothermia failed to improve outcome after severe head injury, and that it was in fact detrimental in patients over the age of 45 . The only patients who had improved outcome were those under the age of 45 who presented already hypothermic.

Methods: A two-year retrospective review of all severe head injuries was performed, with special attention to the use of hypothermia and related outcome.

Results: Data analysis is currently being conducted. Results are pending.

\section{P-182}

S- 100 beta levels: useful for assessment of head trauma patients? First results of a pilot study

E. Schultke, V. Sadanand, M. Kelly, S.-N. Hyat Khan, B.H.J. Juurlink, R.W. Griebel (Saskatoon, Saskatchewan)

Background: S-100 beta serum levels have been shown to constitute a valuable marker in the assessment of cerebral injury. A positive correlation between computer tomography findings after head trauma and S-100 beta serum levels has been demonstrated. If S- 100 beta levels were detectable in urine, similar information might be derived without the necessity for blood sampling. We report the first results from our pilot study.

Method: S- 100 beta urine levels from patients with isolated head trauma have been assayed using an immunoluminometric method. Urine was collected on admission, 12, 24, 48 and 72 hours after admission.
Results: The subunit of the S-100 beta protein was found to be present in the urine of patients after head trauma as well as in that of healthy controls. S- 100 beta urine levels in healthy male volunteers were on average higher than in healthy female volunteers. Increase of S- 100 beta in urine was detected at 48 and $72 \mathrm{hr}$, after an initial plunge below normal first seen at $12 \mathrm{hr}$ in moderate head injury.

Conclusions: The significance of S- 100 beta levels in urine deserves to be further investigated. Higher numbers of patients are required to make statements about the statistic significance of our findings.

\section{P-183}

Traumatic pneumocephalus, epidural hematoma, and intraparenchymal hematoma secondary to diving barotrauma

\section{S. Yip, D.E. Griesdale (Vancouver, British Columbia)}

Background: Intracranial barotrauma secondary to scuba diving is relatively rare. A thorough review of medline revealed a single report in the English literature published before the advent of CT. We report on a patient who developed acute onset headache after a diving ascent.

Results: The patient had no loss of consciousness nor focal neurologic deficits and findings. However, his headache was quite severe, especially on the left side and was refractory to analgesics. A CT scan showed left temporal pneumocephalus with concomitant acute epidural hematoma and intraparenchymal hematoma. Further scans of the temporal bone showed communication between the mastoid air sinus and the intracranial compartment via a small bony defect. A small bubble of air was seen traversing this defect into the cranial vault. The patient had a thorough otolaryngological examination which revealed no perilymphatic fistula. The patient's hospital stay was unremarkable and a follow-up CT scan showed resolution of the intracranial air and blood and the patient was discharged home. Interestingly, the patient returned to the emergency room several weeks later with severe left-sided headache while skydiving. A CT scan showed no reaccumulation of intracranial air nor blood.

Conclusion: Acute pneumocephalus and intracranial blood secondary to diving barotrauma is quite rare. This patient's initial CT scan showed direct communication between his air sinus and the intracranial compartment. This patient's management, as well as a review of traumatic pneumocephalus, will be discussed.

\section{P-184}

Traumatic penetrating orbitofrontal injury with a wooden object: operative, critical care and long-term management

\section{S. Yip, TJ.Zwimpfer (Vancouver, British Columbia)}

Penetrating orbitofrontal injury represents a unique type of penetrating head injury. The unique anatomy of the orbit as well as the mechanism of injury determines the extent of intracerebral and ophthalmological injuries. We describe an interesting case of such injury and its acute surgical management and issues encountered during the management of this patient.

A young male suffered a penetrating orbitofrontal injury from a motorbike accident. A one-inch diameter tree branch entered the brain to a depth of $8 \mathrm{~cm}$ via the right orbit. Craniotomy was performed to extract the tree branch and debride the right frontal lobe. It was noted that the right lobe was preserved upon removal of 
the branch. The patient then underwent repair of his fractured superior orbital roof, duraplasty, craniectomy as well as insertion of ICP monitors.

This patient's course in the ICU was complicated by persistent elevated ICP and extension of frontal necrosis. A second craniotomy was performed for further debridement. Hefnia Alviae was isolated from resected tissue. The patient eventually made a remarkable recovery. On discharge he was alert and oriented, able to read with his right eye, and was able to ambulate on his own. He was transferred to a rehabilitation facility and was discharged home. A cranioplasty was performed five months post injury.

We described a case of penetrating orbitofrontal injury from a wooden object and the surgical and critical care of this patient. Long-term follow-up issues were also discussed.

\section{Pediatric Neurosurgery}

\section{P-185}

\section{Pediatric aneurysms: the Montréal Children's Hospital} experience

\section{J.-F. Chevalier (Montreal, Quebec), W. Choi (Denver, USA);} J.-P. Farmer, J.L. Montes, A.M. O'Gòrman (Montreal, Quebec)

Background: Previous series of aneurysmal SAH reported a $0,5-$ $2,6 \%$ incidence of aneurysms in children. Typically, these affect mainly boys, are more often large or giant, are located on posterior circulation vessels, and are often fusiform or posttraumatic. We report a updated series of nine consecutive patients over 30 years.

Method: Retrospective case series.

Results: The Montréal Children's Hospital emergency department has an average flow of 90000 patients/year. Over the last 30 years, nine children presented with subarachnoidal hemorrhage secondary to cerebral aneurysms. Aneurysms secondary to AVM, mycotic aneurysms and aneurysms of the vein of Galen were excluded. Five initially presented with a Hunt and Hess grade of I/II, two grade III, two grade IV/V. Four children exhibited rebleeding, leading to a preoperative grade $I$ in one, III in 3, and IV/V in five children. Five were clipped, one coiled, one wrapped and one untreated.

Conclusions: Our results go along with the current literature with eight boys involved for only one girl, and a good postoperative evolution despite a poor preoperative grade with six children returning to school unimpaired and one more child attending special classes. Two remained with moderate disability, and one with a poor outcome after rebleeding, bringing him from a grade I to a grade V. Our review does not support the belief of frequent fusiform or posttraumatic aneurysms, only one patient for each category having been seen. Because of the high rebleeding rate with missed diagnosis, a strong index of suspicion must be emphasised.

\section{P-186}

Intracranial complications of frontal sinusitis in children (Pott's puffy tumour)

E. Maroun, J. Jacob, T. Smith, G. Murray, S. Bridger (St. John's, Newfoundland)

Background: Pott's puffy tumour, defined as a scalp swelling and associated intracranial infection, is extremely rare in the antibiotic era.
Methods: The clinical history of three children is presented. All presented with pyrexia, forehead swelling and lethargy. Several intracranial complications (osteo-myelitis, subdural empyema, epidural abscess) due to delayed treatment of frontal sinusitis were successfully managed. Literature is reviewed.

Conclusions: Early recognition and treatment of acute frontal sinusitis with associated frontal periorbital swelling will help to reduce risk of intracranial complications.

\section{P-187}

Incidence of shunt dependent hydrocephalus in children with posterior fossa tumours in the Maritimes

P.D. McNeely, WJ. Howes, R.O. Holness (Halifar, Nova Scotia); S. Walling (Saint John, New Brunswick); V. Mehta (Edmonton, Alberta)

Background: Hydrocephalus requiring long-term cerebrospinal fluid (CSF) diversion is a known complication of posterior fossa tumours in children. Recent experience has suggested a potential role for endoscopic third ventriculostomy (ETV) prior to tumour resection in order to obviate the need for permanent shunt placement. The objective of this study is to examine the incidence of hydrocephalus in children with posterior fossa tumours in our patient population.

Methods: A retrospective review of patients with newly diagnosed posterior fossa tumours treated between 1995 and 2001 at the IWK Health Centre in Halifax, Nova Scotia, and at the Region I Hospital in St. John, New Brunswick was undertaken. Patients undergoing surgery for neoplasms involving the cerebellum and brainstem were included. We excluded patients with tectal plate gliomas as well as patients who did not undergo surgery as part of their treatment.

Results: During the study period, 53 children underwent surgery for posterior fossa tumours. Of these, 38 (72\%) underwent placement of a temporary external ventricular drain at the time of tumour resection. A ventriculoperitoneal shunt was placed in ten patients (19\%) following their posterior fossa surgery.

Conclusions: Although many children with posterior fossa tumours initially present with hydrocephalus, in our experience most ( $81 \%$ ) do not require permanent CSF diversion. In our opinion, the practice of routine ETV prior to posterior fossa tumour resection is not warranted given the overall low incidence of hydrocephalus following posterior fossa tumour resection. Further study to identify risk factors for the requirement of long-term CSF diversion following posterior fossa tumour resection in children is required.

\section{P-188}

Conservative management of a large parietal cephalohematoma

\section{V.Mehta (Edmonton, Alberta)}

Background: Cephalohematomas occur in up to $3 \%$ of newborns following birth and are often a consequence of minor trauma. The majority of cephalohematomas are well-localized, tend to soften and resolve within one month of delivery. Various treatment options have included identifying an underlying cause, observation, aspiration, head wrapping as well as direct surgical repair.

Methods: We present the case of a four-month-old child with a 
large left sided parietal cephalohematoma. This child was born at term via an uneventful delivery and this lesion was completely calcified on imaging. After discussion with the family the pros and cons of treatment were discussed and it was elected to follow the child. It was felt that with the further potential growth of the skull combined with the lesion being present in an area of hair cover that a satisfactory cosmetic appearance could be obtained with no intervention.

Results: After one year of observation both the medical team and the family were satisfied with the cosmetic results obtained.

Conclusions: Large calcified cephalohematomas are uncommon lesions for which there are many treatment options. The natural history of these lesions combined with the growth of the developing skull may allow these lesions to be observed with a satisfactory cosmetic outcome.

\section{P.189}

The association of temporal lobe arachnoid cysts and chronic subdural hematomas in the pediatric population. Cause for concern?

\section{Mehta, K. Aronyk (Edmonton, Alberta)}

Background: Temporal lobe arachnoid cysts represent over $50 \%$ of all intracranial arachnojd cysts. These congenital intraarachnoidal collections of cerebrospinal fluid are generally indolent lesions.

Methods: We reviewed our pediatric neurosurgical database between 1990-2001 and report three cases with chronic subdural hematomas associated with a temporal lobe arachnoid cyst.

Results: Patients 1 and 2 were 14-year-old males that presented with progressive headaches after a minor head injury. Initially both these two patients were treated with burr hole drainage. Both patients represented six and one month later respectively with similar preoperative symptoms and required a craniotomy with fenestration of the arachnoid cyst into the basal cisterns.

Our third patient, a nine-year-old girl was involved in a bike accident, and presented with a history of increasing headaches for two months. She was successfully treated with a craniotomy and fenestration of her arachnoid cyst into the basal cisterns.

Conclusions: Minor trauma may facilitate the development of chronic subdural hematomas among patients with temporal lobe arachnoid cysts. All three of our children presented with an insidious history of increased intracranial pressure and eventually required a craniotomy and fenestration of their cysts into the basal cisterns. Although burr hole drainage may be attempted, in our experience a more definitive procedure is often required.

\section{P-190}

\section{Long-term functional outcome following selective posterior rhizotomy}

S.Mittal, J.P. Farmer, B. Al-Atassi, J. Gibis, E. Kennedy, C. Galli, G. Courchesnes, C. Poulin, MA. Cantin, T.E. Benaroch, (Montreal, Quebec)

Background: Selective posterior rhizotomy is a well-recognized treatment for children with spastic cerebral palsy. Few studies have used quantitative outcome measures to report results beyond three years. The authors analyzed data obtained from the McGill
Rhizotomy Database to determine the long-term functional outcome.

Methods: A multidisciplinary team evaluated the children preoperatively, at six-months and one-year postoperatively. Quantitative standardized assessments of lower limb spasticity, passive range of motion, muscle strength, and ambulatory function were obtained. Of 93 patients who met the entry criteria for the study, 71 and 50 completed the 3-year and 5-year assessments, respectively.

Results: Statistical analysis demonstrated significant improvements in spasticity, range of motion, and functional muscle strength at 1-year. The preoperative, 1-year, 3-year, and 5-year values for the global score of the GMFM were $64.6,70.8,80.0,85.6$, respectively. However, the greatest improvement occurred in dimensions reflecting lower extremity function, where the mean change was $10.1 \%$ at 1 -year, $19.9 \%$ at 3 -years, and $34.4 \%$ at 5 -year in comparison to baseline value.

Conclusions: Significant improvements in lower limb functional motor outcome are seen 1-year after SPR. The improvements persist at 3-and 5-years. We conclude that SPR is valuable in permanently alleviating lower limb spasticity while augmenting motor function.

\section{P.191}

Dynamic cervicomedullary compression without vertebral instability in a patient with Chiari II malformation

\section{A.V. Price (Vancouver, British Columbia), D. Swift (Dallas, USA)}

Background: Cervicomedullary compression symptoms in patients with Chiari type 11 malformations is usually secondary to either neural compression from the overlying dorsal meningeal and bony coverings or bony instability. This is a case report of a myelomeningocele patient with symptoms of cervicomedullary compression without evidence of either. Prior to this case, we were unaware of dynamic neural compression in the absence of vertebral or craniovertebral instability.

Methods: A case study of an 11-year-old male myelomeningocele patient with symptoms of sleep apnea and left digit paresthesias in the setting of a functional ventriculoperitoneal shunt. Examination revealed new bilateral cranial nerve VI palsies, decreased gag reflexes bilaterally, and an unchanged lower extremity exam. A routine cervicomedullary MRI revealed no compression. Flexionextension cervical radiographs failed to demonstrate vertebral instability, however a flexion-extension MRI demonstrated dynamic ventral compression of the brainstem while in the flexed position.

Results: The patient underwent a craniocervical fusion. Postoperatively his sleep apnea symptoms and cranial nerve pareses resolved.

Conclusions: Symptomatic reversible cervicomedullary compression may occur in the absence of vertebral instability. Flexion MRI could be diagnostic in patients with symptoms of cervicomedullary compromise unexplained by other relevant radiological evaluations.

\section{P-192}

\section{Pediatric cavernous sinus trigeminal schwannoma}

J.Spears, J. Rutka, (Toronto, Ontario); W. Hader (Calgary, Alberta)

Background: Trigeminal schwannomas in children are extremely rare. We report a 14-year-old female, without neurofibromatosis, with 
a left cavernous sinus ophthalmic division trigeminal schwannoma.

Method: The patient presented with a three month history of headaches, painless proptosis of the left eye, and diplopia in all directions of gaze. CT and MR imaging of the brain revealed a 2.6 $\mathrm{cm} \times 1.7 \mathrm{~cm}$ lesion involving the left cavernous sinus and posterior aspect of the left orbit. Expansion of the left cavernous sinus with partial erosion of the left superior orbital fissure and anterior clinoid were noted. A left, frontotemporal craniotomy with orbitozygomatic osteotomy and gross total removal of the lesion was performed using microsurgical techniques.

Results: Pathology confirmed a schwannoma. Post-operatively, the patient had diminished sensation in the Vl distribution which was unchanged at six month follow-up. MR imaging at the same time revealed a $6 \mathrm{~mm}$ enhancing lesion which will be followed with serial MR imaging.

Conclusion: To our knowledge this is the first cavernous sinus, ophthalmic division, trigeminal schwannoma reported in the literature in the pediatric age group. Trigeminal schwannomas in the pediatric population are reviewed.

\section{P-193}

Spinal cord retethering after lipomyelomeningocele repair: the Calgary experience

R. Yong. V.M. Houtzager, M.G. Hamilton, S.T. Myles, (Calgary, Alberta)

Background: Retethering of the spinal cord is a common problem after lipomyelomeningocele repair. The purpose of our study was to assess the frequency of symptomatic retethering in patients initially operated on at our institution. The effectiveness of re-untethering and factors associated with symptomatic retethering were also explored.

Methods: The charts and diagnostic imaging of all patients who underwent lipomyelomeningocele repair at the Alberta Children's Hospital between 1983 and 2001 were reviewed.

Results: With a median follow-up of 37.5 months, the frequency of symptomatic retethering in our group of 24 patients was $37.5 \%$. This frequency was similar in patients presenting symptomatically and asymptomatically. Hypalgesia was the most common symptom at initial presentation. Bowel/bladder dysfunction and motor weakness were the most common symptoms of retethering. Repeat procedures were less effective than initial procedures at improving symptoms. Complications $(p=0.007)$ and more laterally situated lipomas $(p=0.034)$ were associated with significantly higher frequencies of retethering.

Conclusions: Patients undergoing lipomyelomeningocele repair are at high risk of symptomatic retethering. In those presenting with neurological symptoms, the benefits of surgery may outweigh this risk. In those presenting asymptomatically, however, our data suggest no clear benefit to prophylactic surgery in light of the unknown natural history of the malformation.

\section{Child Neurology}

\section{P-194}

Lafora disease - an acute presentation and diagnostic challenge S. Alotaibi, B. Minassian, W. Logan, S. Weiss (Toronto, Ontario)

Background: Lafora's disease (LD) is an autosomal recessive progressive myoclonus epilepsy with onset in late childhood or adolescence. We describe a 16-year-old girl who presented with seizures and rapid intellectual deterioration over a period of six months. The diagnosis of LD was delayed due to negative skin, conjunctival and muscle biopsy. The diagnosis was eventually made on detection of the characteristics periodic acid - Schiff (PAS) positive neuronal inclusions (Lafora bodies) from brain biopsy. This was previously reported in several members of a family with Lafora disease in which results of liver and skin biopsies performed during well-advanced disease were often negative.

Method: Electrophysiology studies, neuropathology, positive emission tomography (PET) will illustrate the important aspects of the patient's course.

Results: The diagnosis of Lafora disease was made nine months after presentation in this previously well teenager.

Conclusion: Patients with LD can present with a rapid deterioration. A skin biopsy is the least invasive pathologic method of diagnosis with a very high but not perfect sensitivity. The clinician must have a high index of suspicion when a patient 10-20 years of age presents with intellectual deterioration and intractable seizure disorder despite negative skin and muscle biopsies.

\section{P-195}

\section{Kernicterus in the new millennium}

\section{S. Alotaibi, D. MacGregor (Toronto, Ontario).}

Background: Prevention of bilirubin encephalopathy is based on the detection of infants at risk of developing a significant hyperbilirubinemia. Our objective was to reassess the clinical syndrome of kernicterus in the last decade with more universal availability of neurodiagnostic studies and to review the causal links and outcome.

Methods: The study population included term neonates admitted to The Hospital for Sick Children between January 1990 and May 2000 . The inclusion criteria were total serum bilirubin $>400 \mathrm{mmol} / \mathrm{L}$ at diagnosis and no evidence of hypoxic ischemic encephalopathy. Their charts reviewed for etiology and neurodevelopment outcome.

Results: Twelve neonates (nine males, three females) were identified, bilirubin levels at diagnosis were $477-825 \mathrm{mmol} / \mathrm{L}$. Etiologies were as follows: G6PD (7 patients), dehydration (3), sepsis (1), undetermined (1). Abnormal visual evoked potentials were found in three of nine patients, abnormal brain stem auditory evoked potentials were found in seven of 10 patients, and abnormal EEGs were found in all five patients. Brain magnetic resonance imaging was abnormal in three of four patients.

Conclusions: MRI proven to be useful in the assessment of the structural changes of chronic postkernicteric bilirubin encephalopathy typically showing increased signal in the posteromedial aspect of the basal ganglia. 
Autistic regression in early onset partial complex seizures: a review of eight cases

\section{E. Anagnostou, B. Rosenblatt, F. Andermann (Montreal, Quebec)}

Background: One third of children with autistic spectrum disorder (ASD) develop seizures. The seizures usually present in late childhood, they are usually partial and easily controlled. The goal of this study was to examine the relationship of epilepsy and ASD in patients that develop partial complex seizures before the onset of ASD.

Methods: The files of two pediatric neurologists in Montreal were searched for patients who developed partial complex seizures and then presented with autistic regression.

Results: There were eight children identified with the above method. The age of onset of epilepsy was between five months and 2.5 years. All had normal developmental exams documented by their pediatrician prior to onset of epilepsy and two patients had normal neurodevelopmental exams documented by a neurologist. Within six months of the onset of epilepsy, all children suffered a developmental regression. One year after the onset of seizures, all children were diagnosed with ASD.

Conclusion: We propose that some children with early onset epilepsy are at risk for developing autistic regression. The control of seizures and perhaps the epileptic activity itself may, thus, be very important in these patients.

\section{P-197}

Electrographic asynchronous and asymmetric burst suppression in a child with corpus callosum disease secondary to lymphoma

\section{J. Hukin, A. Davey, P. Wong, C. Parsons, J. Wu (Vancouver, British Columbia)}

Background: Burst suppression is characterised by bilaterally synchronous and symmetric bursts of electrical activity followed by intervals of attenuation. The neurological prognosis is guarded in the absence of a reversible cause. Our case developed coma associated with asynchronous burst suppression following low dose craniospinal radiation.

Case: A 13-year-old female was diagnosed with $\mathrm{Ki}-\mathrm{l}$ anaplastic large cell lymphoma, without CNS involvement. Chemotherapy: vincristine, cyclophosphamide, prednisone, daunomycin, Lasparaginase, intrathecal methotrexate, and adriamycin. She developed severe back pain and paraparesis. MRI scan showed bulky metastatic disease involving brain and spinal cord, including corpus callosum. Steroid therapy was initiated with cranial irradiation at $180 \mathrm{cGy}$ per fraction. Cognitive status fluctuated and after the third fraction she became comatose. The EEG showed asynchronous burst suppression. Symptoms improved within 72 hours of treatment with high dose steroids and chemotherapy. Neuroimaging corroborated a marked decrease in tumor bulk. There was gradual improvement in neuro-cognitive status with persistent neurologic deficits. She died one month later of progressive disease.

Conclusion: Low dose cranial irradiation may induce coma in the presence of intracranial bulky tumour. Burst suppression coma may be reversible when secondary to tumor and radiation effects. We describe the first report of asynchronous burst suppression associated with tumor.

\section{Are children who have ischemic strokes hypertensive?}

Selina Hune, Anita Allen, Marianne Sofronas, Trish Domi, M.E. Holt-Brockenbrough, Gabrielle deVeber (Toronto, Ontario)

Background: The relationship between hypertension and childhood stroke has not been established.

Objective: To determine the prevalence and predictors of hypertension in children following stroke.

Methods: Ambulatory blood pressure (BP) recordings from children aged one year to 18 years seen in the Stroke Clinic between January 1998 and June 2001 at HSC were reviewed. BPs from agematched children with epilepsy (but no stroke) were used to control for the 'white coat' response to clinic visits. BP values greater than the 90 th percentile of published age, height and gender normed BP values were classified as 'hypertension'.

Results: 'Hypertension' was present in $37(58 \%)$ of 64 stroke patients, and 25 (42\%) of 60 control patients (Chi Square: $P=0.052$; $\mathrm{OR}=1.9,95 \% \mathrm{CI}: 0.93$ to 3.91$)$. Predictors of 'hypertension' were presence of migraine $(4 / 4=100 \%)$, moya moya $(3 / 3=100 \%)$, sickle cell $(2 / 4=50 \%)$ and other vasculopathy $(8 / 9=89 \%)$.

Conclusions: Children have elevated BP following stroke, especially in the setting of cerebral vasculopathies. Whether elevated BP is causative or a sequel to stroke, or results from underlying diseases causing stroke, is unknown. Further studies are needed to determine the mechanisms of elevated BP, as well as explore the correlation between increased $\mathrm{BP}$ and stroke recurrence.

\section{P-199}

\section{Acute childhood encephalitis and the Epstein-Barr virus}

A. Doja, D. MacGregor, R. Tellier, A. Bitmun, H. Heurter, M. Petric, E. Lee Ford-Jones (Toronto, Ontario)

Background: Many neurologic manifestations of Epstein-Barr virus (EBV) infection have been documented including encephalitis, aseptic meningitis, transverse myelitis, and Guillain-Barré syndrome. These manifestations can occur alone or coincident with the clinical picture of infectious mononucleosis.

Methods: Since 1994, The Hospital for Sick Children has maintained a prospective registry of all children admitted with acute encephalitis. Our aim was to summarize all cases of EBV encephalitis compiled from $1994-2000$.

Results: Fifteen children were identified as having encephalitis and evidence of EBV infection. This evidence consisted of convincing EBV serology and/or positive cerebrospinal fluid (CSF) PCR. One patient had symptoms similar to classic infectious mononucleosis; all others had a non-specific prodrome including fever $(100 \%)$ and headache $(66 \%)$. A majority $(66 \%)$ had seizures and most had electroencephalograms showing a slow background (73\%). Many demonstrated CSF pleiocytosis $(60 \%)$ and $53 \%$ had abnormal MRI findings. Two patients died but $80 \%$ were neurologically normal or had only mild deficits at follow-up.

Conclusions: Overall, patients with EBV encephalitis do not show symptoms of infectious mononucleosis. The diagnosis can best be made by the presence of convincing EBV serology, positive EBV PCR and the absence of other potential etiologic agents. Overall, patients tend to do well with few neurologic sequelae. 
P-200

\section{Familial paroxysmal nonkinesigenic dyskinesia response to lorazepam}

J. Dooley, K. Gordon (Halifax, Nova Scotia)

Background: Familial paroxysmal nonkinesigenic dyskinesia (Mount and Reback dyskinesia) is characterized by episodes of dystonia and/or choreoathetosis. Effective therapy for these episodes is not currently available.

Methods: Two sisters from a large kindred involving three generations with familial paroxysmal nonkinesigenic dyskinesia were studied. Both children were taking prophylactic clonazepam, which partially controlled their symptoms but they continued to have attacks once or twice per week. During these 1-2 hour episodes they were unable to remain at school. Both sisters omitted their usual dose of clonazepam on the evening prior to testing and were sleep deprived in order to precipitate an event. The children were videotaped and were asked to report when their symptoms improved. Patient 1 (a 10-year-old) had episodes characterized by dystonia and chorea. Patient 2 (an 8-year-old) had attacks which were primarily dystonic.

Results: Both girls had typical attacks during a day-care admission. They received sublingual lorazepam $(0.1 \mathrm{mg} / \mathrm{Kg})$ and noted a resolution of their symptoms within 5 minutes. No adverse effects were seen.

Conclusions: Patients with familial paroxysmal nonkinesigenic dyskinesia have been reported to respond favorably to prophylactic therapy with clonazepam, or valproate. To-date, no efficacious and safe acute abortive therapy is available for breakthrough events. We have demonstrated that sublingual lorazepam may have a rapid beneficial effect in these patients.

\section{P-201}

A child with alternating hemiplegic migraine, focal seizures and transient hemispheric cortical edema

C.D. Hahn, S. Weiss, D.L. MacGregor, S. Blaser, (Toronto, Ontario)

Background: Hemiplegic migraine is a syndrome characterized by recurrent attacks of hemiplegia and headache. Approximately $50 \%$ of sporadic and familial cases are associated with missense mutations in the neuronal calcium channel gene CACNA1A.

Methods: Clinical records and MRI studies of a child with hemiplegic migraine were reviewed.

Results: A Jamaican girl presented at age 5,6 and 7 with three attacks of headache, prolonged hemiplegia and subsequent focal seizures. The first two attacks were right-sided, the third left-sided. MRI during each attack demonstrated cortical edema with mass effect and gyral effacement of the hemisphere contralateral to the hemiplegia. MRA was normal. EEGs demonstrated slowing over the affected hemisphere. Recovery from the hemiplegia occurred over 1-4 months. An MRI between attacks demonstrated mild volume loss of the previously affected hemisphere. CSF examination, echocardiography, immunologic markers for vasculitis, organic and amino acids, and testing for MELAS syndrome were normal. Mutation analysis of the CACNAIA gene is pending.

Conclusions: We hypothesize that the hemispheric cortical edema demonstrated in this child was caused by local inflammation, altered vasoreactivity, or by the prolonged focal seizures. The subsequent development of mild hemispheric volume loss suggests that there has been irreversible cerebral injury despite good clinical recovery.

P-202

Absence seizures: retrospective review of outcome and factors that predict good prognosis

\section{Huh, D. Buckley (St. John's, Newfoundland)}

Background: Absence epilepsy comprises 11-15\% of childhood epilepsies and occurs in approximately 1 in 10000 individuals. We reviewed our population of children with absence seizures in order to determine if they equate with the trends published in literature.

Methods: The Janeway Child Health and Rehab Center is the only tertiary care pediatric facility in the province of Newfoundland and Labrador. The neurology department has a complete database of all children seen with epilepsy in this province. All children with absence seizures were identified through the Neurology Database.

Results: 31 female and 28 males were identified with absence seizures, however, 19 female and 21 males were eligible for analysis due to exclusion secondary to incomplete data or ongoing medication use. $76 \%$ of the males and $53 \%$ of the females went into remission.

Ages at onset ranged from six months to 15 years with a majority being between 4-12 years of age. The younger the age of onset, the higher the chance of remission. Two thirds of those with onset before the age of four years and only a third of those with onset after age of eight years had remission.

Generalized tonic-clonic seizures were present in $21 \%$ of our population and were correlated with a lower likelihood of remission.

As valproic acid is used as the first-line treatment in childhood absence seizures in our center, comparison of anticonvulsants was not reliable. The duration of treatment, however, was not found to be a significant variable in outcome. Those treated less than two years had similar outcome to those treated for greater than two years. The rate of discontinuation (0-6 months) did not have an impact on recurrence of seizures.

A typical EEG pattern $(2-4 \mathrm{~Hz}$ spike and wave) was not predictive of better outcome. An abnormal EEG at discontinuation, however, did predict a worse outcome.

Conclusion: Despite our small study population, we were able to surmise that positive predictive factors include; male sex, age of onset $<8$ years, no co-morbid generalized tonic-clonic seizures, and normal EEG at discontinuation of anticonvulsants. This is consistent with previously published literature.

\section{P-203}

\section{Variable phenotype of vanishing white matter disease}

\section{Joshi, F. Booth (Winnipeg, Manitoba)}

Background: Vanishing white matter disease is an autosomal recessive leukoencephalopathy, recently linked to chromosome $3 q$. This leukodystrophy has a better prognosis for life expectancy as well as higher functions. Clinical course and MRI findings of two cases, one thought to have spastic diplegia for many years, are described.

Clinical presentation: Both patients presented at an early age 
with unusual constellation of irritability, incoordination and lethargy triggered by seemingly minor infections or trauma. One patient remained static for about seven years during which time he was considered a spastic diplegic. The other patient had repeated deteriorations with minor trauma. Cognition was relatively preserved in both patients and MRI showed diffuse, bilaterally symmetrical white matter abnormalities with cystic changes, sparing the cortex and deep gray structures.

Other causes of leukodystrophy were ruled out by extensive testing including EEG, EMG, NCV, metabolic screen, enzyme assays, fibroblast assays, CSF studies, and muscle biopsy.

Conclusion: Vanishing white matter disease has a variable phenotype and must be considered in the differential diagnosis of leukodystrophy. Genetic disorders with a phenotype similar to cerebral palsy may remain static for many years before eventual progression.

\section{P.204}

Evaluation of pediatric CNS malignancies with 99Tcmsestamibi SPECT

\section{A. Kirton, H. Kloiber, J. Rigel, J.E.A. Wolff (Calgary, Alberta)}

Background: SPECT imaging has the potential to add valuable information to the diagnosis and management of CNS malignancy. Radioactive tracers including $99 \mathrm{mTc}$-Sestamibi (99Tcm-MIBI) have been shown to be sensitive markers for brain tumors, however, their role in imaging children is poorly defined.

Methods: We undertook a pilot study of 29 pairs of $99 \mathrm{Tcm}-\mathrm{MIBI}$ and MRI images from 20 children to explore the clinical usefulness of this tracer in CNS malignancy.

Results: Tumor types that took-up 99Tcm-MIBI included brain stem glioma, fibrillary astrocytoma, other low grade astrocytomas, and glioblastoma multiforme. Astrocytomas were the most consistently positive type of tumor. This method of nuclear imaging was not only able to identify tumor presence, but could also identify changes in the same tumor over time. Some correlation between histological grade and 99Tcm-MIBI uptake was observed. Several tumors evident on MRI were not visible on 99Tcm-MIBI SPECT including craniopharyngioma, medulloblastoma, and optic glioma.

Conclusion: Results suggest that this modality is a potentially useful tool in the diagnosis and management of CNS malignancies in children, particularly higher grade astrocytomas.

\section{P.205}

Breathing irregularities in sleep with vagal nerve stimulation therapy in children with refractory epilepsy

L. Nagarajan, P. Gregory, J. Maul, S. Stick, S. Ghosh, M. Lee, P. Walsh, (Perth, Australia)

Objective: To assess changes in respiratory pattern in sleep with VNS therapy.

Background: VNS therapy is an accepted adjunctive option in the management of patients with refractory epilepsy (RE). We have a cohort of 19 children with VNS therapy for RE. Reported breathing irregularities in sleep (BIS) by the parents of one child resulted in further investigation with a polysomnography study (PSG) in this patient and subsequently in seven others.
Design/Methods: Overnight sleep PSG was undertaken in eight patients using a Compumedics data acquisition and software package, consistent with standard practice. The studies were analysed by a neurologist and a respiratory paediatrician. The parents of all patients in the cohort were questioned specifically about any BIS with VNS.

Results: In 7/8 BIS were evident, concordant with VNS stimulus being on. In the child with no BIS, the VNS was set at the rapid cycling mode. In the first child studied there was a reduction in respiratory rate (RR) and air flow, whereas in the other six there was an increase in the RR, and a decrease in air flow. In some, there was a drop in oxygen saturation of $1-3 \%$ (never greater than $4 \%$ ) in association with the BIS. No one had $\mathrm{CO}_{2}$ retention. The BIS with VNS were not associated with arousals.

Conclusion: During sleep VNS resulted in BIS in the majority of the children studied. In one child with reduction in RR, changing the stimulus parameters (frequency of the stimulus and intensity, signal on time) did not improve the BIS. Only four parents reported observing changes in breathing pattern in sleep with VNS. BIS may be clinically significant in some patients with VNS and this effect needs to be investigated further.

\section{P-206}

Epidemiological study of pediatric brain tumors in Newfoundland: changing outcomes over three decades

C. Burton (Saint John, New Brunswick), A. Prasad (Winnipeg, Manitoba); D. Buckley, C. Pushpanathan, J. Hand, J.C. Jacob, F. Maroun (St. John's, Newfoundland); L. Jardine (London, Ontario)

Background: We reviewed our experience of pediatric brain tumors over 1967-1998 using the cancer registry maintained the Janeway Child Health Centre.

Methods: Retrospective audit of medical records.

Results: Of 189 cases identified there were 98 males, and 91 females (M:F 1:1.08). The mean age at diagnosis was 111.2 mos. (95\% CI 103.4 to 119.1 mos.) with a majority of cases clustering in the Avalon Peninsula. There was no significant relationship between age at diagnosis and tumor location (One way ANOVA $\mathrm{p}=0.36$ ). Headaches, vomiting, visual impairment, and gait disturbance vere amongst the most frequently encountered symptoms reflecting raised intracranial pressure. On histology, astrocytomas, medulloblastoma, glioma, and ependymoma, were most common tumor types. Combined modality treatment using surgical resection and radiation $(\mathrm{n}=52)$ was most frequently used. Mean survival time was 178 months (95\% CI, 156.4-400 mos.) from diagnosis. Kaplan Meier analysis of survival time indicates longer survival for patients diagnosed after cranial imaging modalities (CT, MRI) became available in Newfoundland. After treatment, a significant proportion (58.3\%) were left with single or multiple neurodevelopmental disabilities.

Conclusion: The study shows an improved survival following availability of cranial neuroimaging facilities in Newfoundland, but significant morbidity in survivors with pediatric brain tumors. 
P-207

Manifesting heterozygosity in a family with carnitine palmitoyltransferase II deficiency due to the serine 113 leucine mutation

M.F.Rafay, E. G. Murphy, I. Tein, (Toronto, Ontario); P. Kaufmann, S. DiMauro, (New York, USA); J.D. McGarry, (Dallas, USA)

Background: Carnitine palmitoyltransferase II deficiency is an autosomal recessive disorder. Homozygotes typically present with recurrent myoglobinuria and heterozygotes are usually asymptomatic.

Methods: We describe and correlate the clinical, biochemical and mutation analysis of a family in which the affected proband is homozygous for CPTII deficiency, due to the Ser 13Leu mutation.

Results: Proband, 18-year-old female, presented at age 3-years with episodic myalgia, weakness and myoglobinuria, precipitated by infection. Investigations revealed ictal creatine kinase (CPK) 3600 IU/L and 33\% residual CPTII activity in cultured skin fibroblasts. Her 23-year-old non-identical twin-brothers presented with milder phenotypes consisting of muscle stiffness following prolonged exercise, lightheadedness after prolonged fasting, but no overt pigmenturia. Both had interictal CPKs up to $662 \mathrm{IU} / \mathrm{L}$ and $37 \%$ residual CPTII activities. Her parents and 12-year-old brother are asymptomatic. An elder sister suffered recurrent pigmenturia and died at eight-years from an episode of myoglobinuria with renal failure. She presumably had CPTII deficiency but had not been investigated. Molecular analysis revealed that the proband is homozygous for the Serl13Leu mutation consistent with her low residual CPTII activity. Her parents are heterozygous and have intermediate CPTII activities of $50-70 \%$, consistent with their heterozygous state. Her younger brother is homozygous normal with $83 \%$ residual activity. The symptomatic twin-brothers are heterozygous but demonstrated unexpectedly low CPTII activities of $37 \%$, which may explain their phenotype.

Conclusion: We postulate genetic, environmental and sex-linked factors for this manifesting heterozygosity.

\section{P.208}

Rare good oultcome following acute hypoxic-ischemic thalamic injury in term newborns: predictive clinical features

E.H.Roland, K.J. Poskitt, M.A. Sargent, A. Hill (Vancouver, British Columbia)

Background: The pattern of acute hypoxic-ischemic cerebral injury in term newborns which involves predominantly central gray matter (thalami, basal ganglia) with relative sparing of cortex and subcortical white matter is generally recognized to be highly predictive of poor neurological outcome. However, there is a spectrum of severity and factors associated with good outcome are not well-defined.

Methods: In an ongoing study of term newborns with hypoxicischemic encephalopathy who had thalamic edema on computed tomography ( $n=57$ ), only four children had minor neurological abnormalities on follow-up (3-8 years of age). We identify clinical and radiologic features associated with good outcome.

Results: Minor abnormalities at follow-up included: tremor (1), clumsiness (3), mild dystonic posturing (2). All were born "flat" with Apgar score of 4 or less at 5 minutes and required positive pressure ventilation for $<30$ minutes after birth. All had moderate encephalopathy with duration of seizures $<48$ hours controlled by phenobarbital. Three had no evidence of injury to systemic organs; one had mild renal dysfunction. All were breast feeding within eight days. Neuroimaging abnormalities were less marked and restricted to thalami and basal ganglia.

Conclusions: Good outcome occurs rarely following acute, perinatal hypoxic-ischemic thalamic injury. Predictive clinical factors include moderate encephalopathy with rapid recovery of feeding difficulties and lack of involvement of systemic organs.

\section{P-209}

\section{Pontocerebellar hypoplasia type 1: new leads for an earlier} diagnosis

M.S. Salman, S. Blaser, J.R. Buncic, C.A. Westall, E. Heon,

L. Becker (Toronto, Ontario)

Background: Pontocerebellar hypoplasia $(\mathrm{PCH})$ type 1 is a rare disease characterized by $\mathrm{PCH}$ and anterior horn cell degeneration. Death results from respiratory failure. The oldest reported child died at the age of 26 months.

Methods: Case study of two siblings with PCH. The diagnosis was made on autopsy after the death of the second sibling at 40 months from respiratory failure and the finding of anterior horn cell degeneration on autopsy. The older sibling died at 14 months from pneumonia following a clinical course similar to his sister.

Results: Both siblings had significant global developmental delay with initial axial and peripheral hypotonia. Peripheral hypertonia with brisk reflexes developed later. Extensive investigations in the second sibling ruled out mitochondrial and other metabolic diseases. Genetic testing for Friedreich's ataxia, NARP, SMN, SCA 1,2,3,6,7,8 gene abnormalities was negative. Sleep study showed mixed central and obstructive sleep apneas. The electroretinogram (ERG) showed abnormal rod-cone response.

Conclusions: Our cases call for the expansion of the phenotype for this disease. Survival may be prolonged with emergent spasticity. The presence of abnormal ERG response in infants with PCH may aid in the earlier diagnosis of this disease, and provide new insights into its pathogenesis.

\section{P-210}

Multifocal myoclonus following group A streptococcal infection P.Smyth, D.B. Sinclair (Edmonton, Alberta)

Background: Movement disorders as a post-infectious phenomenon of group A streptococcal infections have been reported, and thought to occur on an autoimmune basis. We describe an unusual case of focal myoclonus following strep throat infection.

Methods: Clinical description and chart review.

Results: A 10-year-old boy developed focal myoclonus involving his right arm and shoulder one week after a strep throat infection treated with penicillin. His MRI was normal, and he initially responded to clonazepam but did not sustain a response. The myoclonus eventually spread to involve all limbs and trunk, becoming multifocal over the next few weeks. He did not have choreoathetosis of Syndenham's chorea. He was given one course of IVIG, and became asymptomatic after treatment. He has remained 
symptom free for eight months following IVIG treatment.

Conclusions: Various neurologic symptoms have been reported following group A streptococcal infections in children. These neurobehavioural abnormalities may be mediated through antineuronal antibodies. Our case demonstrates multifocal myoclonus as a post-streptococcal autoimmune phenomenon. To our knowledge, only two other cases of post-streptococcal myoclonus have been reported in the literature. Recognition of this unusual condition as a manifestation of autoimmune post-streptococcus disease in children is essential to avoid overinvestigation and to ensure early treatment.

\section{P-211}

Encephalopathy of idiopathic hypereosinophilic syndrome due to demyelination?

\section{E. Wassmer, C. Hahn, S. Weiss, O.C. Snead III (Toronto, Ontario)}

Background: Neurologic manifestations of idiopathic hypereosinophilic syndrome (HES) are peripheral neuropathy, focal CNS deficits and encephalopathy. The pathologic mechanism of HES encephalopathy (HESE) is unknown.

Method: We reviewed the literature and present a three-year-old girl with HES and recurrent progressive encephalopathy.

Results: Our patient with HESE presented with irritability and reduced consciousness. MRI showed bright T2-weighted areas in several vascular territories (ACA, MCA, PCA). She improved with steroid treatment. However, she relapsed after steroids were weaned and presented with transverse myelitis: urine retention, hypotonia, areflexia and weakness of lower limbs. This evolved into an encephalopathy with unresponsiveness, absent oculocephalic responses, cornea and gag reflex and she required ventilation. MRI showed ring enhancing lesions in the conus of the spinal cord, basal ganglia and temporal lobes bilaterally and later in thalami and the entire brain stem.

Conclusion: The focal neurologic deficits of HESE is thought to be due to ischaemia caused by micro-embolism. However reports show disappearance of lesions in repeated MRI-scans and patients responding to steroid-treatment. Another possible pathology could be demyelination. HESE could be due to demyelination.

\section{P-212}

Valproic acid-associated weight gain in older children and teens with epilepsy

\section{E. Wirrell (Calgary, Alberta)}

Purpose: To determine the incidence and predictors of weight gain in older children and teens treated with valproate for epilepsy.

Methods: Subjects aged 10 through 17 years who commenced valproate between $01 / 01 / 96$ and 31/12/00, and had documented weight and height measurements at medication initiation and one follow-up visit, were retrospectively identified. Exclusion criteria were follow-up less than two months, discontinuation of valproate within two months, concurrent therapy with medication known to affect weight or gastrostomy feeding. Body mass index (BMI) was calculated both at initiation and either time of discontinuation of valproate or last follow-up, and stratified into four categories; underweight (BMI $<5$ th percentile), appropriate weight (BMI 5-85th percentile), potentially overweight $(\mathrm{BMI}>85$ th-95th percentile) and overweight (BMI >95th percentile). Potential predictors of change in $\mathrm{BMl}$ on valproate were examined.

Results: Forty-three subjects (23 M, $20 \mathrm{~F})$ ivere identified. In 25 $(58 \%)$ the follow-up BMI on valproate was higher than expected (median increase 2.0, 25-75th percentile 1.0-6.4), while in 16(37\%) it was lower (median decrease 1.2, 25-75th percentile 0.2-1.8). Seventy-nine percent remained in their same BMI category, and $14 \%$ moved up at least one category. The only predictor of an overweight $\mathrm{BMI}$ at follow-up was a potentially overweight or overweight BMI at initiation $(p<0.0002)$. Two factors tended to predict an increase in BMI on therapy; normal neurocognitive status $(p=0.06)$ and primary generalized seizure type $(p=0.07)$.

Conclusion: While marked weight gain was uncommon, over half of older children and adolescents treated with valproate experienced an increase in their BMI above that expected. Subjects who were overweight at onset were more likely to remain overweight at follow-up, but were not more likely to have an increase in BMI on therapy than those of appropriate iveight.

\section{Multiple Sclerosis}

\section{P.213}

\section{Intensive monitoring of open-label use of mitoxantrone for multiple sclerosis}

M. Keegan, D. Sneve (Rochester, USA)

Background: Mitoxantrone was recently approved in the United States for multiple sclerosis following reports of safety and efficacy in Europe. Given the potential for serious side effects close monitoring of open label usage is necessary.

Merhods: Seventeen consecutive patients treated with mitoxantrone underwent intensive monitoring including regular complete blood counts, urinalysis, and repeated transthoracic echocardiograms.

Results: At treatment initiation, 13 patients $(76.5 \%)$ were continuing to have relapses of MS while 4 (23.5\%) had a solely progressive course. All patients were initially treated with $12 \mathrm{mg} / \mathrm{m}^{2}$ intravenously every three months. Currently, patients have been treated with a median of three doses (range 1-5) for a median duration of 12 months (range 3-17) comprising a mean cumulative dose of $38.5 \mathrm{mg} / \mathrm{m}^{2}$. Of the 13 patients having attacks prior to initiation, four $(30.8 \%)$ had a new relapse during treatment with three requiring corticosteroids. MRIs done on four patients $(23.5 \%)$ during treatment revealed two having new active lesions. Three patients $(17.6 \%)$ required a dose reduction but none required discontinuation. One patient $(5.8 \%)$ had significant neutropenia that was transient. Five patients $(29.4 \%)$ had urinary tract infections, one developed cellulitis, one mild alopecia and none developed pneumonia. One discontinued treatment due to lack of efficacy. One patient had a transient reduction in cardiac ejection fraction but no patients had clinically significant cardiomyopathy.

Conclusions: Mitoxantrone may be administered with relatively few serious complications. Intensive monitoring of its use may contribute to its safe use. 


\section{P.214}

T2 relaxation measurements of in vivo water content and myelin water content in normal appearing white matter and lesions in multiple sclerosis

C. Laule, I.M. Vavasour, J. Oger, D.K.B Li, D.W. Paty, A.L. MacKay (Vancouver, British Columbia)

Background: Magnetic resonance imaging measurements of T2 relaxation can provide estimates of total and myelin water content in vivo; this will help in understanding the pathology of MS.

Methods: T2 relaxation data were acquired using a 32-echo (TE $=10-320 \mathrm{~ms}$ ) experiment from $33 \mathrm{MS}$ patients and 15 controls. Normal appearing white matter (NAWM) regions of interest (ROls) were drawn in all subjects and in 185 MS lesions. Relative water content was the ratio of signal in the ROI to an internal grey standard. Myelin water content was the ratio of signal with $\mathrm{T} 2$ below $50 \mathrm{~ms}$ to the total $\mathrm{T} 2$ signal.

Results: On average, relative water content was $1.8 \%$ higher and myelin water content was 15\% lower in NAWM of MS compared to controls. Lesions in white matter had, on average, a $7.7 \%$ higher total water content and $50 \%$ lower myelin water content than NAWM.

Conclusions: NAWM in MS has more total water and less myelin water than controls. A simple model of white matter suggests that edema alone cannot account for the observed $1.8 \%$ increase in water content and $15 \%$ decrease in myelin water content. Therefore, we believe that the white matter cerebral NAWM undergoes diffuse demyelination.

\section{P-215}

Adverse drug reactions: discrepancies between clinical trials and post marketing surveillance studies: MS and betainterferon-1a

\section{H. Tremlett, J. Porciuncula, K. Ho, D. Paty, E. Yoshida, J. Oger} (Vancouver, British Columbia)

Background: In MS clinical trials, liver enzyme elevations were reported in up to $27 \%$ of patients treated with beta-interferon-la $\left(\right.$ Rebif ${ }^{\circledR}$ ). The frequency of this adverse event in clinical practice was examined. A report of liver failure subsequent to treatment with Rebif initiated the study.

Methods: A retrospective review of MS patients at UBC followed up for at least six months after initiating Rebif as 'first-line' therapy was carried out. Previous or current IFNB trial patients were excluded.

Results: 215 MS patients were eligible and 208 charts were available. Surveillance serum AST/ALT (3 monthly for one year, yearly thereafter) were performed in 185 patients. Of these, 163 (88\%) had normal baseline results. $71 / 163$ patients $(43.6 \%)$ developed at least one abnormal liver enzyme during treatment, 48/163 (29.4\%) were at least twice the upper normal limit. Characteristics associated with liver abnormalities (LiA) included: male gender (the LiA group comprised of $39.6 \%$ (19/48) males vs. $15.7 \%(18 / 115)$ in the non-LiA group; OR=3.5 (95\%Cl: 1.6 to 7.6$)$ ) and disease duration (DD) (mean $=12.6$ and 9.6 years, respectively; $\mathrm{p}=0.036$ ). Baseline EDSS score, race, MS course and age were not statistically significant between the LiA and non-LiA groups.

Conclusions: Reasons for discrepancies between clinical practice and trials could be multifactorial, including different inclusion criteria and definitions of 'raised' liver enzymes. Male gender and DD could be contributory. Continuing pharmacovigilance is warranted and will be extended to all the beta-interferons.

\section{P-216}

Evolution of new multiple sclerosis lesions: what extra information can novel magnetic resonance techniques give us?

I.M. Vavasour, K.P. Whittall, A.L. Mackay, D.K.B. Li, D.W. Paty (Vancouver, British Columbia)

Background: Novel magnetic resonance techniques such as T2 relaxation and magnetization transfer (MT) are now being investigated as tools which provide more specific information about pathology.

Merhods: Seven MS patients were scanned five times over one year. From this, five MR parameters were calculated: absolute water content (WC), myelin water content (MWC), mean T2, mean TI and magnetization transfer ratio (MTR) for each of 24 new lesions as well as contralateral normal-appearing white matter (NAWM).

Results: WC of new lesions increased by an average of $5 \%$ when the lesion first appeared. In follow-up, WC returned towards the prelesion value but was still $2-3 \%$ higher. MWC decreased to $4.5 \%$ from $6 \%$ when the lesion first appeared and stayed low on subsequent scans. MTR also decreased when the lesion first appeared and remained low. These changes were more pronounced in the centre of the lesion where they persisted, whereas the periphery tended to resolve in later months. All MR parameters from NAWM were constant over time.

Conclusions: This is the first study where absolute water content and myelin water content were measured serially in MS lesions. Changes persisted in the centre of the lesion, but resolved in the periphery.

\section{P-217}

Albertans with insulin-dependent diabetes mellitus (IDDM) are at high risk for multiple sclerosis (MS)

\section{S. Warren, K.G. Warren, L. Svenson (Edmonton, Alberta)}

Background: Epidemiological research indicates that the prevalence of IDDM is significantly elevated in MS patients and somewhat elevated in their relatives; but no research has examined whether MS is elevated in IDDM.

Methods: IDDM patients attending weekly teaching seminars at the University of Alberta's Metabolic Day Care Centre over one year completed a brief questionnaire, including family illness history and number of immediate family members at risk. The Z-test of proportions was used to compare MS prevalence in IDDM patients and their relatives to MS prevalence in Alberta.

Results: Of the 269 IDDM patients who participated, three had MS themselves (two males, one female). Twelve others reported relatives with MS (one father, one mother, one brother, four sisters, zero children, zero grandparents, one uncle and six aunts), for a total of $14 \mathrm{MS}$ cases in 4848 family members. Therefore, the prevalence of MS in IDDM patients was I,115/100,000 and it was 289/100,000 in their relatives. The most recently published MS prevalence rate for Alberta is 216/100,000. Consequently, MS prevalence in IDDM patients is 5.2 fold that of the general population, which is significant. MS prevalence in IDDM patients' relatives is 1.3 fold 
that of the general population, not significant.

Conclusions: The apparent two-way relationship between IDDM and MS suggests several possibilities: common genes, a common environmental cause or that a metabolic disorder is involved in MS.

\section{P-218}

\section{Delayed evolution of recurrent transverse myelitis into} relapsing neuromyelitis optica

\section{D.M. Wingerchuk (Scottsdale, USA)}

Background: Neuromyelitis optica (NMO; Devic's syndrome) consists of optic neuritis and acute myelitis with clinical, laboratory and imaging features distinct from multiple sclerosis (MS). It typically follows a relapsing course. Acute "transverse" myelitis (TM) is usually a monophasic event but case series have described a recurrent form with an uncertain relationship to MS and NMO.
Methods: Cases series.

Results: Four adults presented with cervical or high thoracic TM. Spinal cord magnetic resonance (MR) imaging demonstrated increased $\mathrm{T} 2$ signal extending over three to four vertebral segments; brain MR was normal or showed nonspecific white matter lesions. Cerebrospinal fluid was either normal or showed a lymphocytic pleocytosis (up to $76 \mathrm{WBC}$ ); one patient had a single oligoclonal band. During follow-up (3-11 years), each patient experienced recurrent myelitis $(3,4,4$, and 11 relapses). Each patient then developed unilateral optic neuritis, meeting NMO diagnostic criteria. Brain MR findings remained normal or nonspecific.

Conclusions: Relapsing NMO may begin with recurrent episodes of TM separated by many years before the first event of optic neuritis. Treatment of recurrent TM with regimens reported to be of benefit in NMO, such as azathioprine plus prednisone, may be appropriate for attack prevention and require further study.

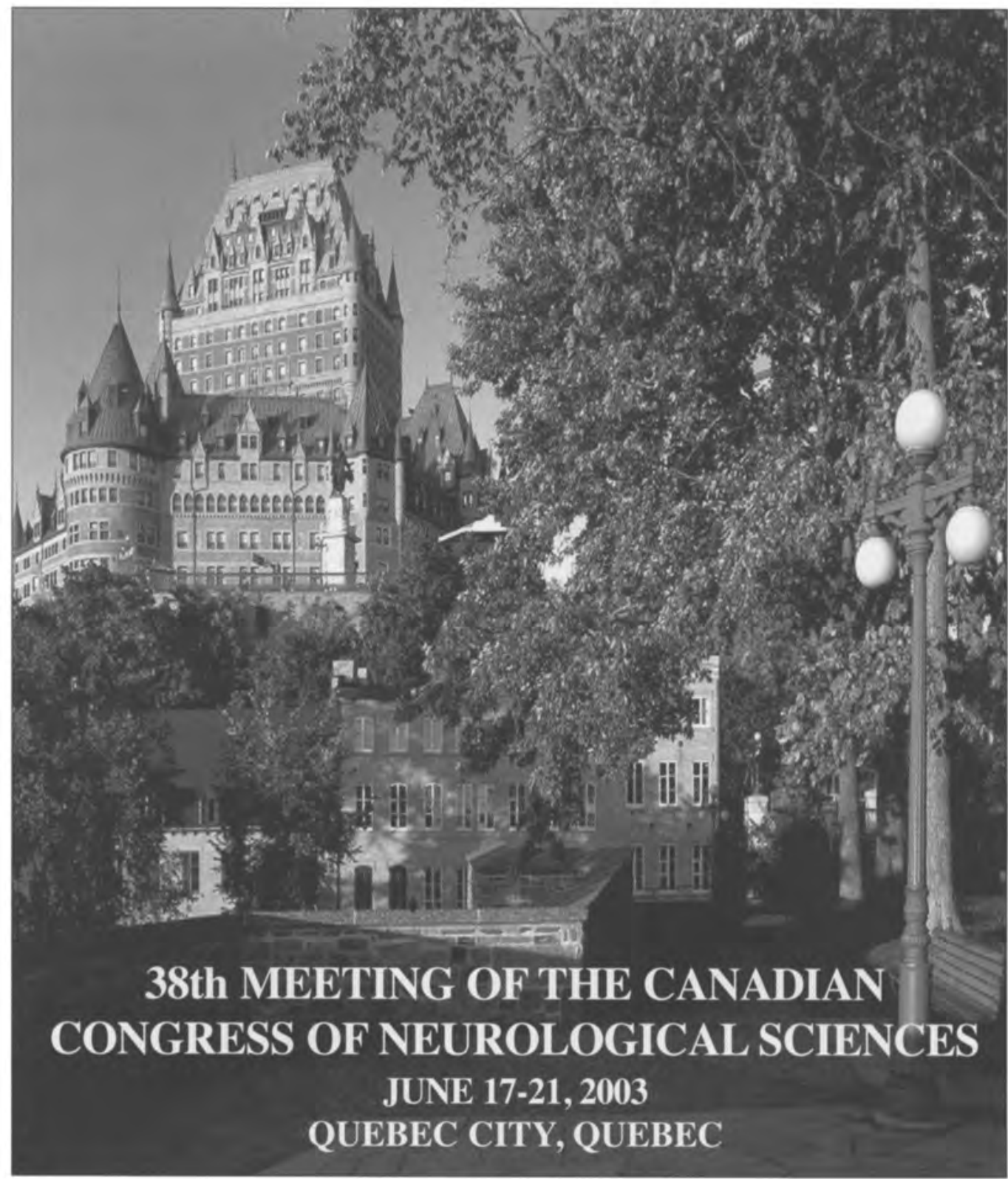

Suppl. $1-$ S87 


\section{INDEX}

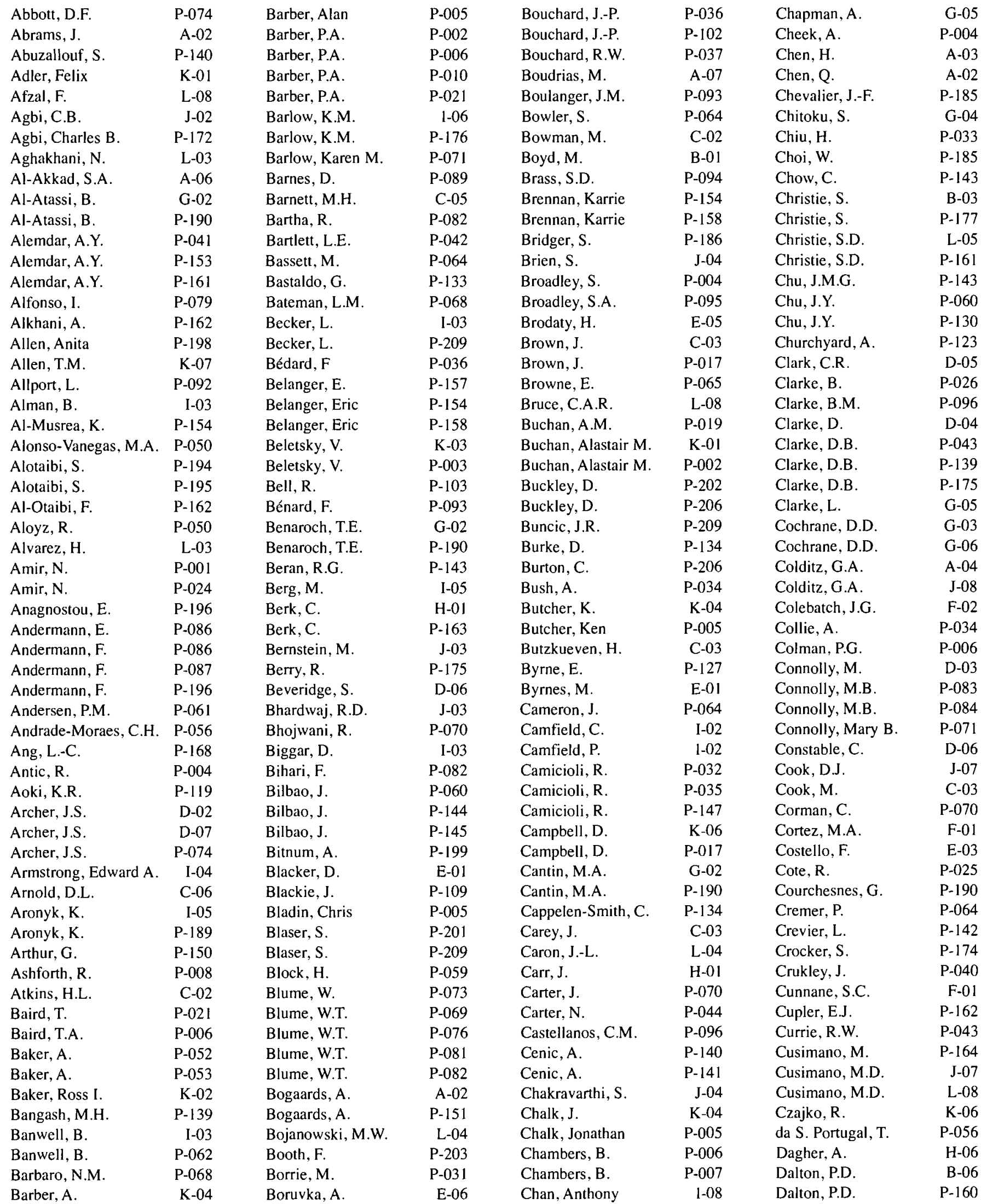




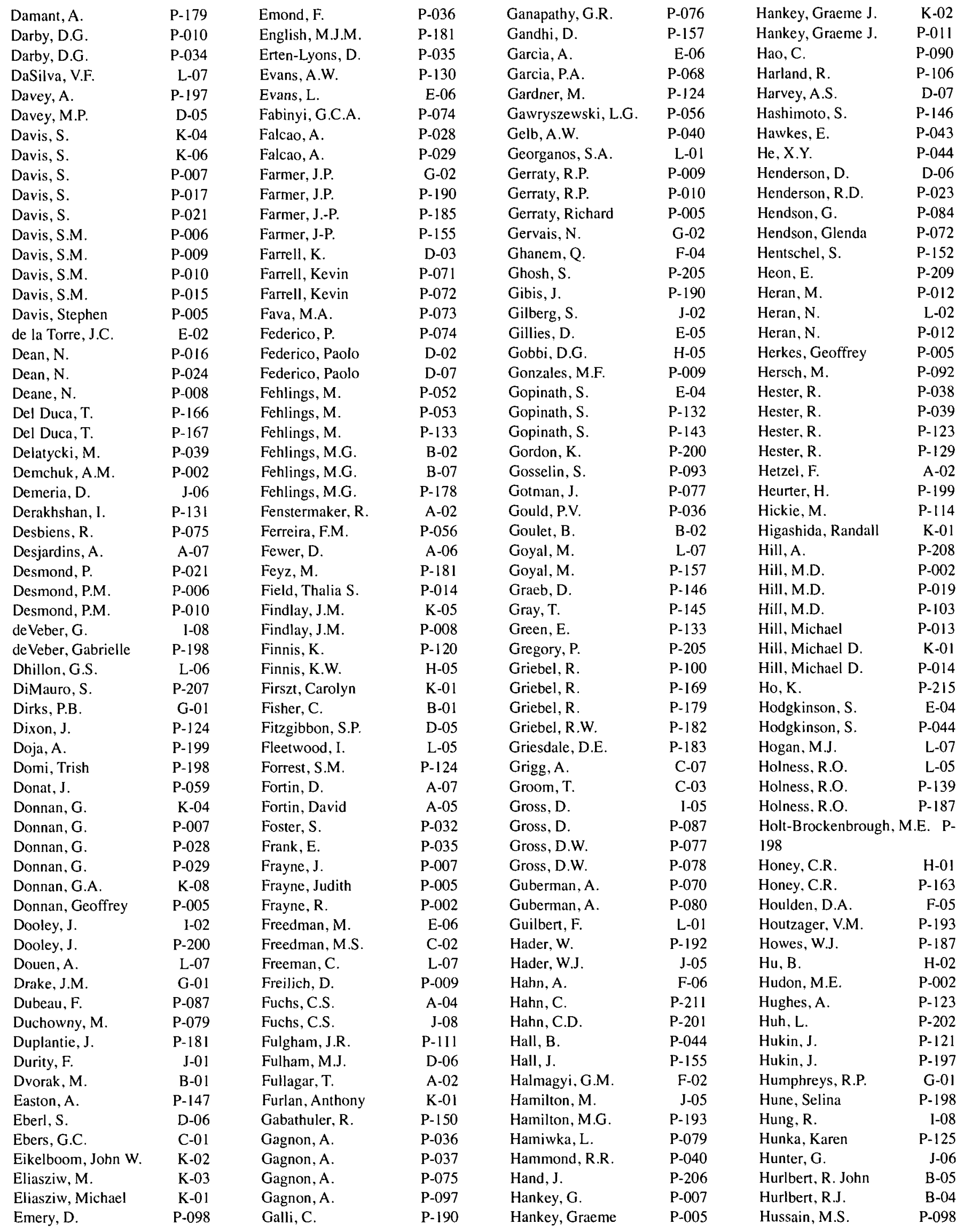




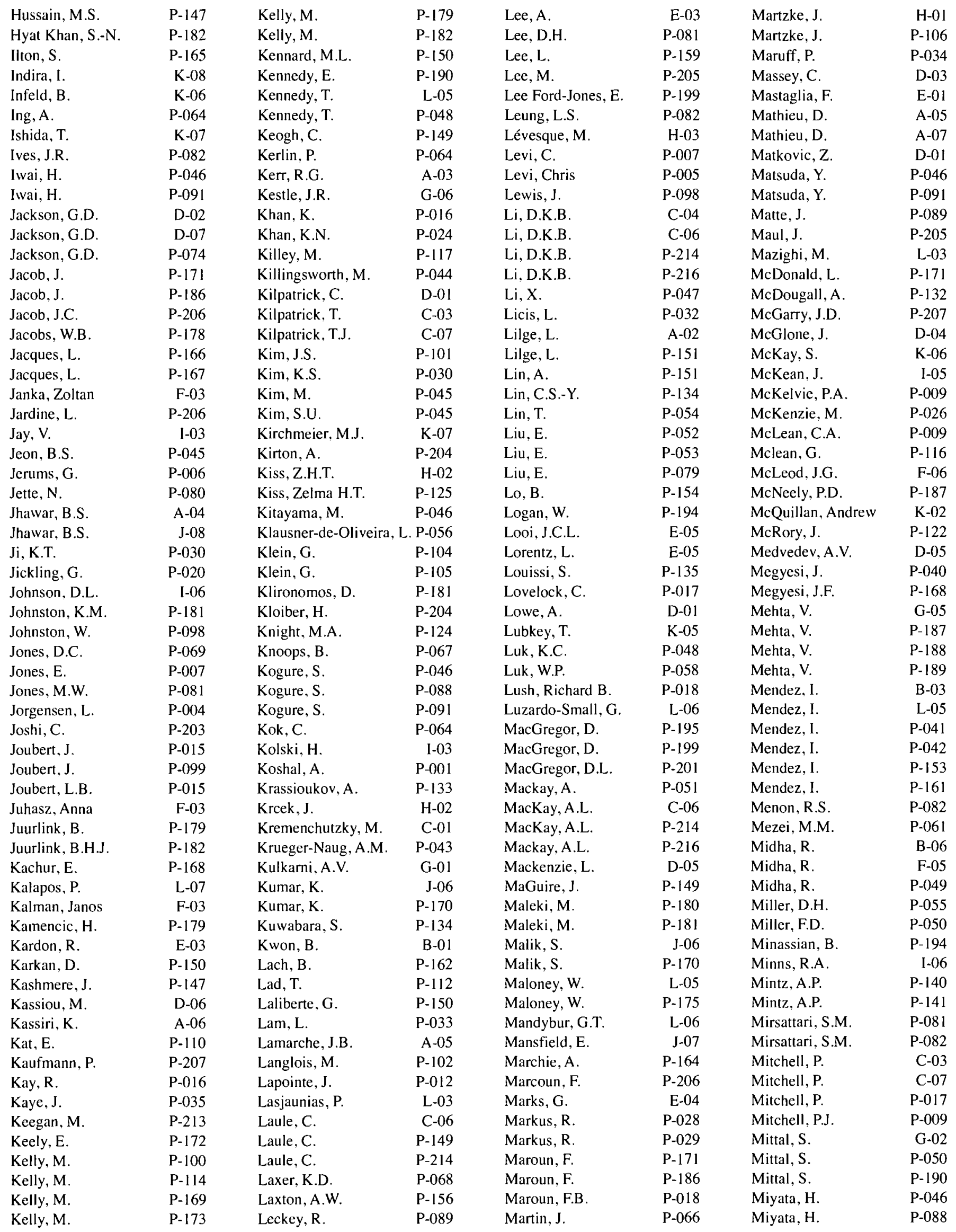




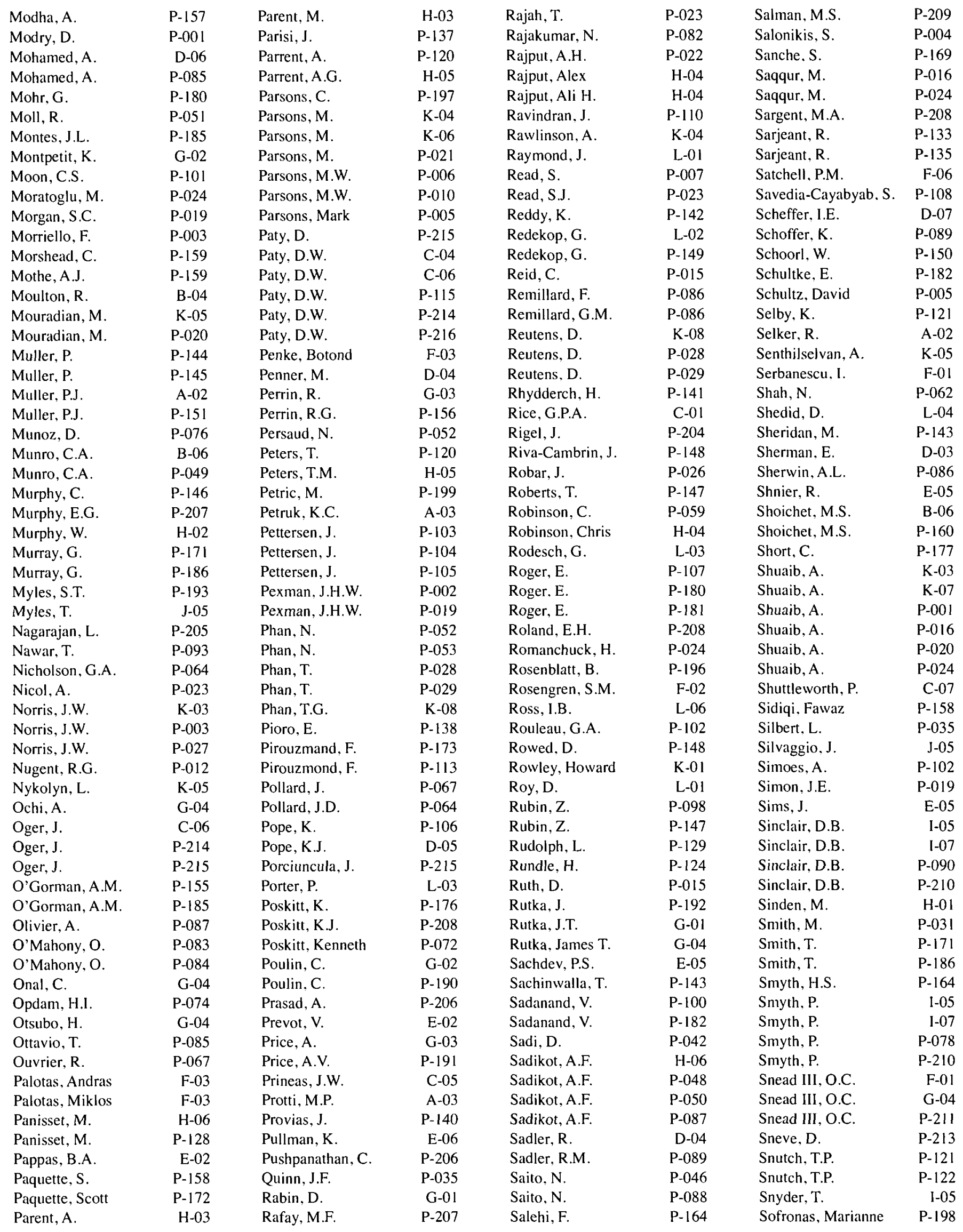




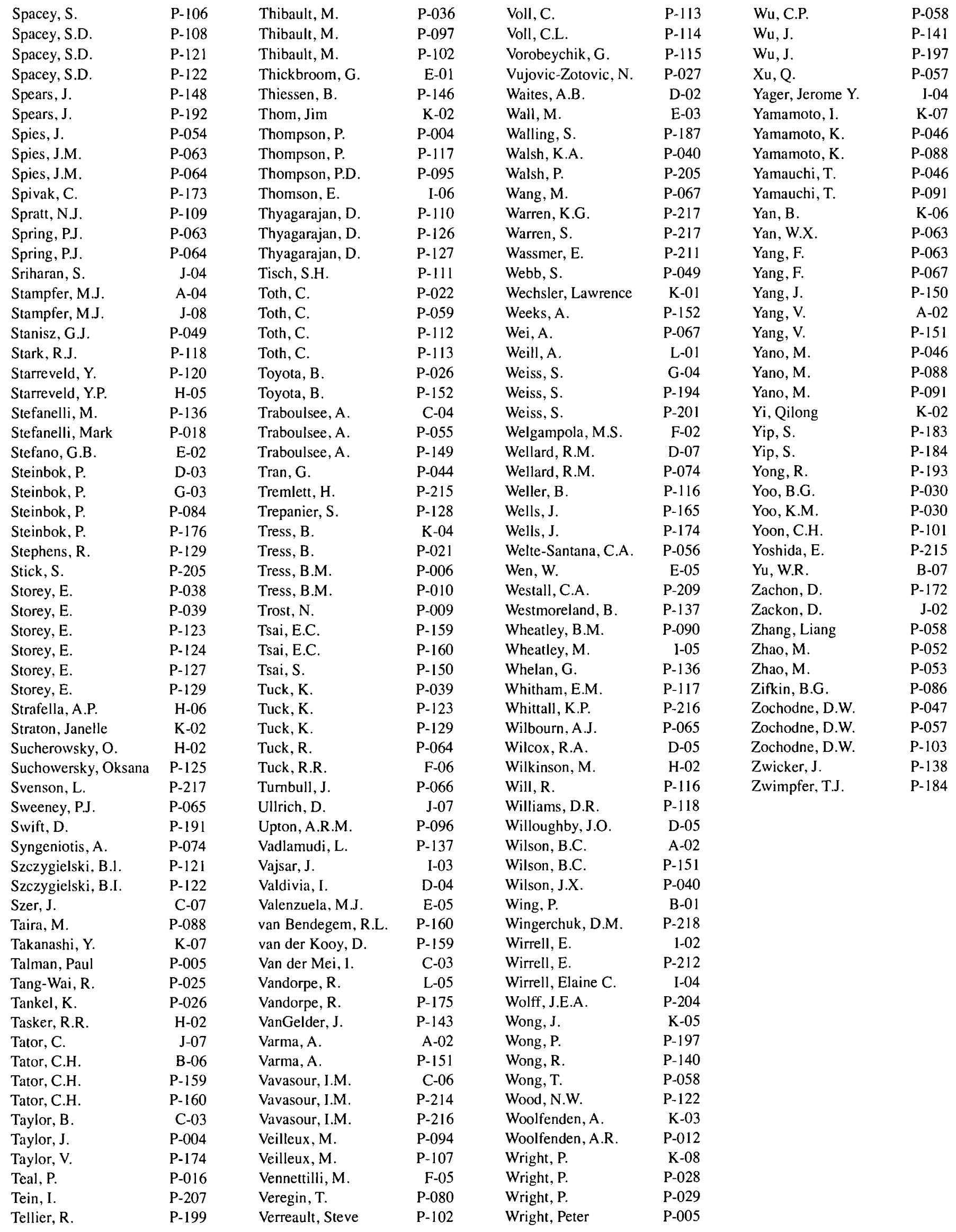




\section{CANADIAN ASSOCIATION OF NEUROSCIENCE NURSES \\ JUNE 13- 15, 2001 \\ HALIFAX, NOVA SCOTIA}

\section{Posters}

1. Treatment for Craniosynostosis: Facilitation of Parental Decision Making

Susan Neufeld RN MN; CNS Children's Neurosurgery, Stollery Children's Hospital; Nicole Lerourneau RN PhD; Assistant Professor, Faculty of Nursing, $U$ of Alta, Jane Drummond $R N$ PhD; Professor, Faculty of Nursing, $U$ of Alta. Edmonton, Alberta.

2. Stereotaxy Illustrated

Marie-Helene Tetreault

3. Normal Perfusion Pressure Breakthrough after AVM Repair: A Case Study

Frankie Wong RN BN ACCN; Instructor of Staff

Development,Clinical Neurosciences Unit; Foothills Medical

Centre, Calgary, Alberta.

\section{Neuroendovascular Procedures}

Caroline Allaire RN BSCN ; Staff Nurse, Claudia Zanchetta RN MN; ACNP, Neurosurgical /Trauma ICU, St. Michael's

Hospital Toronto, Ontario

5. New Frontier in Neurosurgery

Thelna Velasco RN, O.R., Division of Neurosurgery; TWH Division; University Health Network; Yolanda Balatbat RN, CPN (C) Clinical Educator, TWH Division; University Health Network.

6. Endoscopic Third Ventriculostomy as a Treatment for Hydrocephalus

Herta Wai-Ham Yu; RN MN-ACNP; CNS; Nurse Practitioner, Abby Varughese; RN BScN; Staff Nurse; Seetha

Padmanabhan; RN; Clinical Support-Neurosurgery, Maria Lamberti-Pasculli RN Research Nurse; Neurosurgery; Hospital for Sick Children, Toronto, Ontario.

7. A Comprehensive Oral Care Program in a Complex Chronic Care Rehabilitation Hospital

Daniela Cahuas RN BScN MSc GNC (C) Clinical Nurse Educator: The Riverdale Hospital, Toronto, Ontario,
8. Improving Post Operative Pain Management for Patients Undergoing Spinal Fusion with Instrumentation

Ha Quach RN BScN Staff Nurse, Helen Fong RN BScN Staff, Frans-Edna Ilceus RN Staff; Neurosurgery, McGill University Health Centre-Montreal Neurological Hospital Site, Montreal, Quebec.

9. Classical Trigeminal Neuralgia: A Surgical Perspective Debbie Filipchuk RN CPN (C) Staff Nurse Neurosurgery; $U$ of Alta.Hospital, Edinonton, Alberta.

10. Thorns and Roses: A Personal Account of a Nursing Research Project

Pauline Weldon RN BN CNN ( C ) CCRC, Co-ordinator MS Clinic QE II HSC Halifax, Nova Scotia.

11. Gugliemi Detachable Coil in the Treatment of Cerebral Aneurysm; A New Interventional Initiative in a Community Based Hospital

Milagros Santos RN BSN Staff Nurse, Fiorella Paula RN BSN CNN (C) Staff Nurse; Neurosurgery Trillium Health Centre Mississauga, Ontario.

12. Severe TBI: Tracking and Improving Patient Outcomes in Acute Care

Yonne Hall RN; Edith Jasinski RN; Agnieszka Obiedzinska $R N$; Valentine Valenzuela RN; Staff Nurses Neurosurgery; Sunnybrook and Women's Health Sciences Centre, Toronto; Diane Duff RN PhD; Professor of Nursing Centennial College, Toronto, Ontario.

13. Demystifying Creutzfeldt Jakob Disease Roshan Punja RN BSN Staff Nurse, Christine Rowe RN BSN Staff Nurse; Neurosurgery, TWH Division of University Health Network Toronto, Ontario.

14. Family Centred Care-Partners in Care

Sigrun Roennbeck RN Staff Nurse Paediatric Neurosciences, $B C$ Children's and Women's Health Centre Vancouver, British Columbia. 